\title{
Oxygen K-edge \\ X-ray Absorption Spectra
}

Federica Frati 


\section{Oxygen K-edge X-ray Absorption Spectra}

ISBN: 978-94-6423-338-4

Author: Federica Frati

Layout and printing: ProefschriftMaken www.proefschriftmaken.nl

(c) Federica Frati, The Netherlands 2021

All rights reserved. No part of this publication may be reproduced in any form or by any means, electronically, by print or without written permission of the copyright owner. 


\title{
Oxygen K-edge X-ray Absorption Spectra
}

\author{
Zuurstof K-rand Röntgenabsorptie Spectra
}

(met een samenvatting in het Nederlands)

\section{Proefschrift}

ter verkrijging van de graad van doctor aan de

Universiteit Utrecht

op gezag van de

rector magnificus, prof.dr. H.R.B.M. Kummeling, ingevolge het besluit van het college voor promoties

in het openbaar te verdedigen op

woensdag 1 september 2021 des middags te 2.15 uur

door

\section{Federica Frati}

geboren op 23 augustus 1992

te Umbertide, Italië 


\section{Promotor:}

Prof. dr. F.M.F. de Groot

\section{Copromotor:}

Dr. M. Hunault

This doctoral thesis was accomplished with financial support from the European Reasearch Counsil (ERC Advanced Grant 340279) 
Forsan et haec olim meminisse iuvabit

(Virgil, Aeneid, I, 203) 

The aim of this thesis is to critically review and theoretically investigate oxygen $\mathrm{K}$ edge absorption spectra in oxide molecules and solids.

The research making up the content of this thesis in Chapter 1, Chapter 2, Chapter 4 and Chapter 6 is the result of an extensive team work aiming to explore the available theoretical and experimental data on oxygen is XAS spectra on molecular and solid systems. We discuss important aspects of various detection routes, highligth the differences and benefits of the available theoretical models and explore the applicability on the study of catalytic reactions. Emphasizing both observational and theoretical aspects, this work provides a critical perspective on oxygen $\mathrm{K}$ edge absorption spectra. Chapter 3 and Chapter 5 aim at detailed investigations of two popular approaches to the modelling of $\mathrm{K}$ edges in molecular and solid systems: the coupled cluster hierarchy of methods and the DFT-based $\triangle \mathrm{SCF}$. The results of Chapter 3 and Chapter 5 test the performances of the computational methodology chosen for the simulation of $\mathrm{K}$ edges in formaldehyde and derivatives and alkaline earth metal oxides and contribute to a further understanding of the electronic structure of the investigated systems. 



\section{CONTENTS}

I INTRODUCTION

1 INTRODUCTION 3

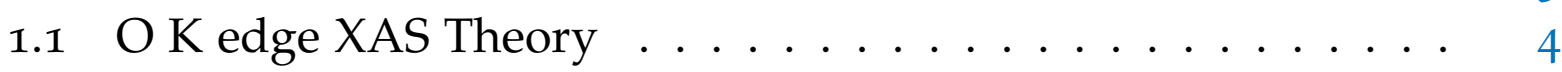

1.1.1 Density Functional Theory (DFT) ......... 5

1.1.2 Beyond the one particle approximation ....... II

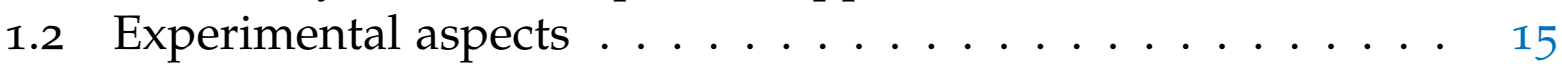

1.2.1 Sources for $x$-ray absorption spectroscopy . . . . . . 15

1.2.2 Detection techniques ............ 17

II ATOMIC AND MOLECULAR SYSTEMS 25

2 ATOMIC AND MOLECULAR SYSTEMS 27

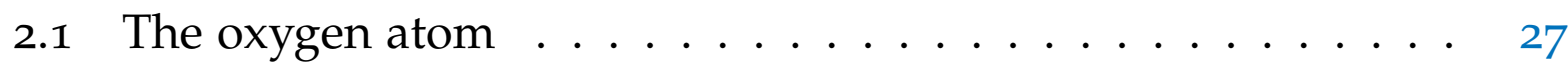

2.1.1 Oxygen ions ................ 29

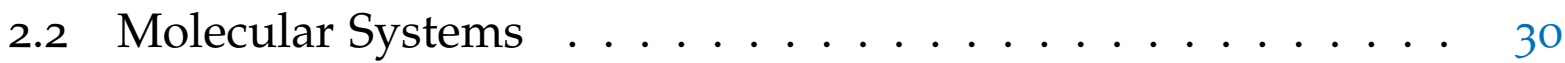

2.2.1 The binary molecules $\mathrm{CO}, \mathrm{NO}$ and $\mathrm{O}_{2} \ldots . . . .30$

2.2.2 Molecules with three atoms ......... 35

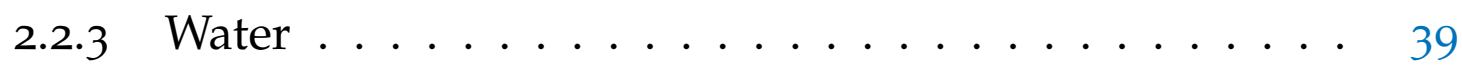

2.2.4 Bio-organic molecules ............ 41

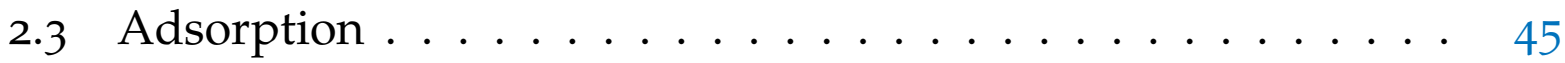

2.3.1 Atomic adsorption .............. 45

2.3.2 Molecular adsorption ............. 47

2.4 Analysis of catalytic reactions . . . . . . . . . . 49

3 COUPLED CLUSTER 51

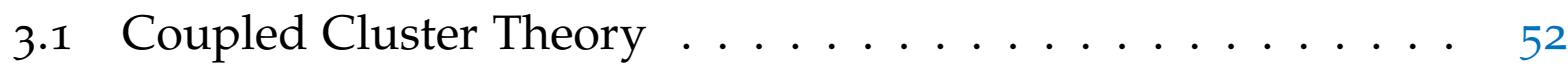

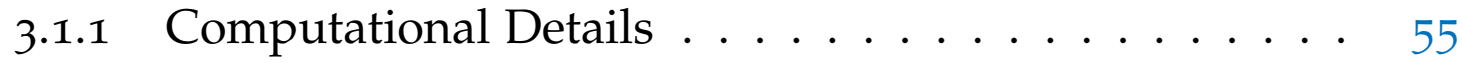

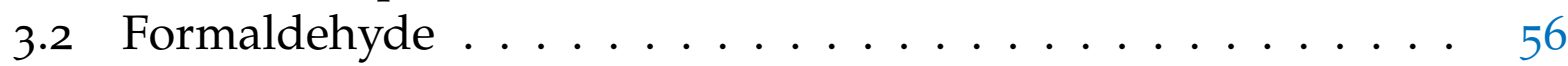

3.3 Formic acid, formyl fluoride and carbonyl fluoride . . . . . 67

3.4 Vibronic structure of the bands ........... 7 . . . . . .

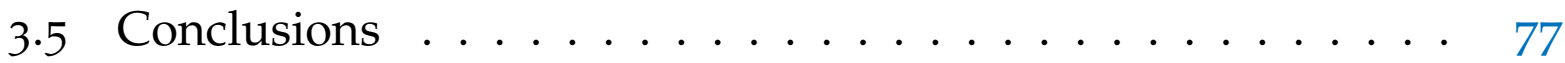

Appendix Chapter III $\quad 79$

III SOLID SYSTEMS 93

4 S AND P-OXIDES 95

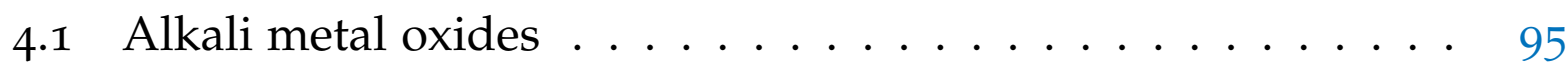

4.2 Oxides with p-electron systems . . . . . . . . . 96 
4.3 Overview of p-element oxides . . . . . . . . . . . 98

5 ALKALINE EARTH METAL OXIDES 109

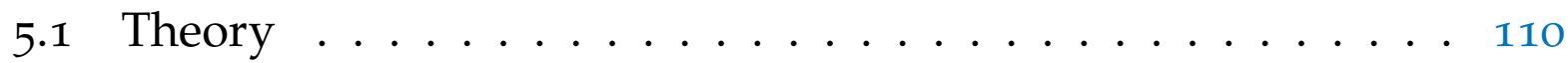

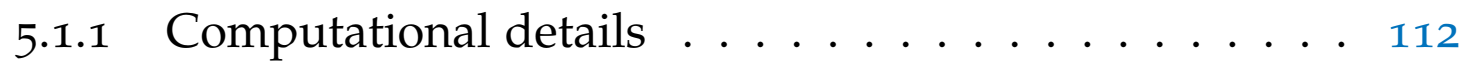

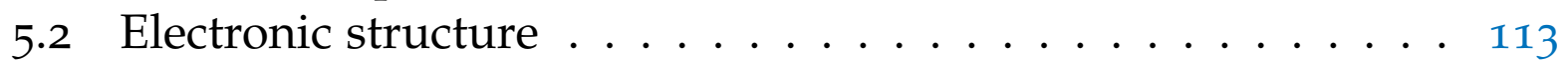

5.2.1 Partial density of states (PDOS) ..........................

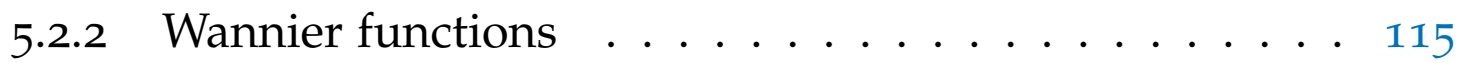

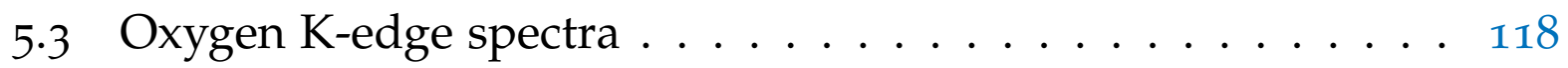

5.3.1 $\mathrm{MgO} \ldots \ldots \ldots \ldots . \ldots \ldots 18$

$5.3 .2 \mathrm{CaO} \ldots \ldots \ldots \ldots . \ldots . \ldots . \ldots . \ldots 19$

$5.3 .3 \mathrm{SrO} \ldots \ldots . \ldots . \ldots . \ldots . \ldots 120$

$5 \cdot 3.4 \mathrm{BaO} \ldots \ldots \ldots \ldots . \ldots . \ldots . \ldots 121$

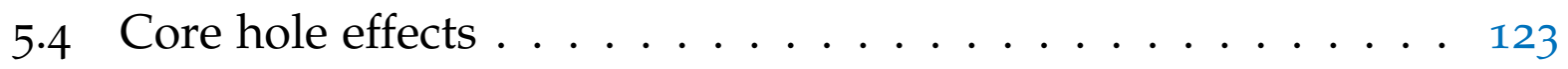

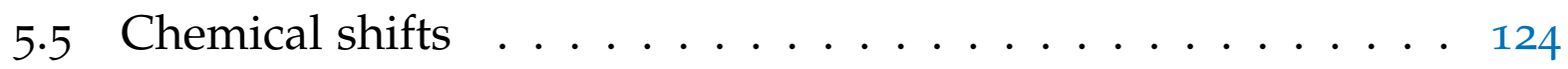

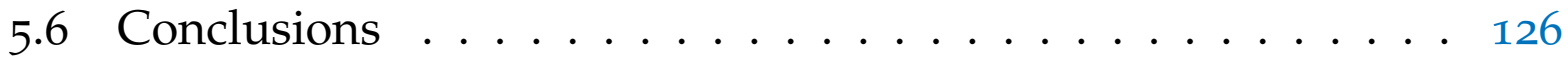

6 D AND F-OXIDES $\quad 127$

6.1 General considerations of 3 d-element oxides . . . . . . 128

6.1.1 The effects of crystal field, exchange and covalency . . 128

6.1.2 Binary oxides and oxidation state trends ..... 130

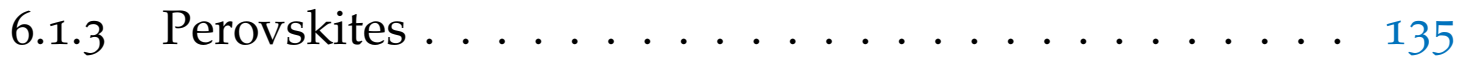

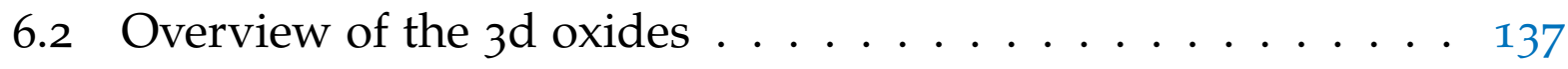

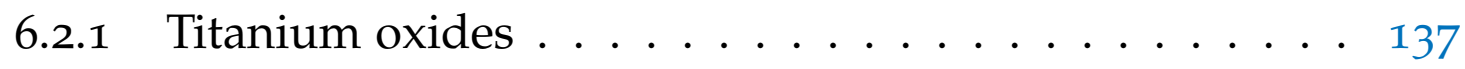

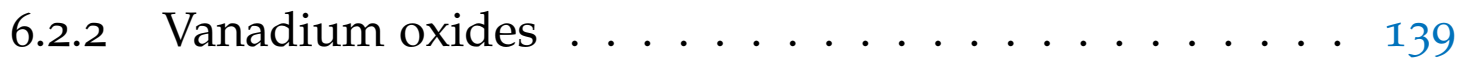

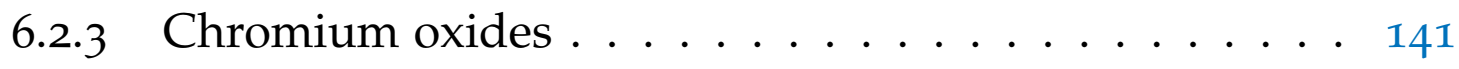

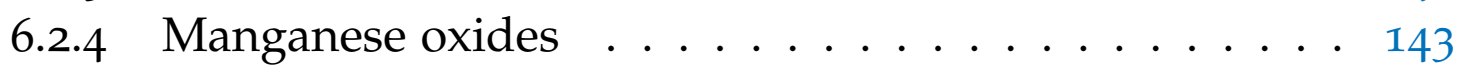

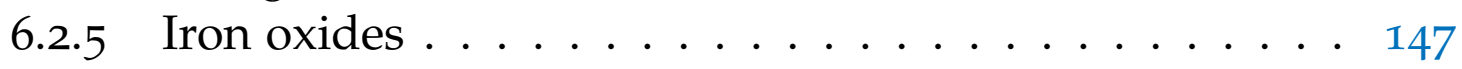

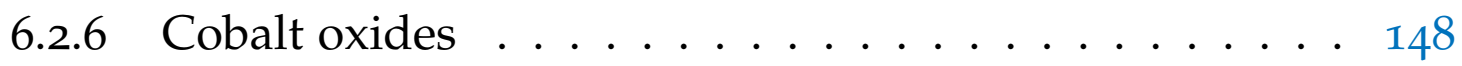

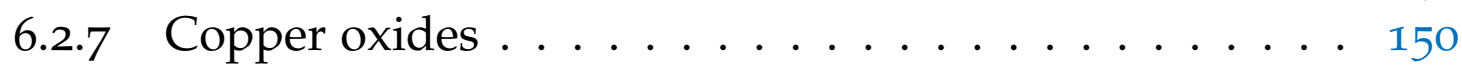

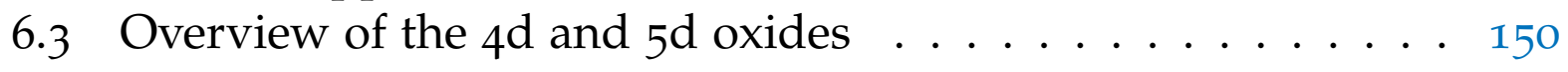

6.3.1 4d transition metal oxides ........... 152

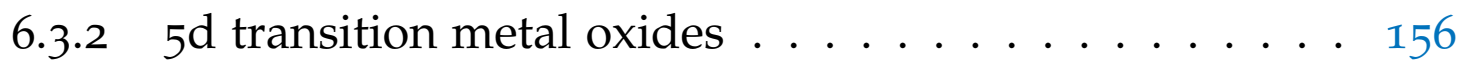

6.4 General considerations of the $4 \mathrm{f}$ oxides ........ 160

6.4.1 Lanthanum oxides .............. 162

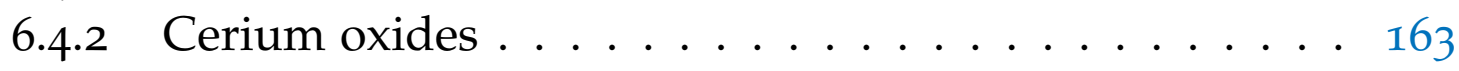

6.5 General considerations of the 5 f oxides . . . . . . . 164

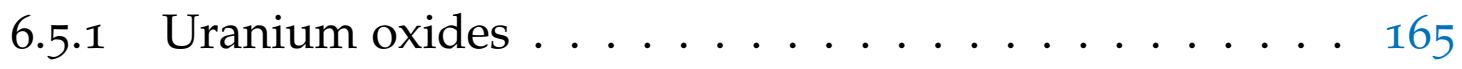

7 SUMMARY AND OUTLOOK 169

$\begin{array}{ll}\text { Publications by the author } & 179\end{array}$

$\begin{array}{ll}\text { Acknowledgments } & 181\end{array}$ 
About the author 183

BIBLIOGRAPHY

185 



\section{Part I}

\section{INTRODUCTION}

In this thesis we discuss oxygen $\mathrm{K}$ edge $\mathrm{x}$-ray absorption spectra of both molecules and solids.

The thesis is divided in three parts: in part I an overview of oxygen $\mathrm{K}$ edge theory and the main theoretical approximations adopted for the modelling of XAS spectra are discussed together with the main experimental aspects of oxygen $\mathrm{K}$ edge $\mathrm{x}$-ray absorption measurements, including $x$-ray sources, monochromators and detection schemes.

Part I is based on the paper: F. Frati, M. O.J.Y. Hunault, and F.M.F. de Groot. "Oxygen K-edge X-ray Absorption Spectra." Chemical Reviews 120.9 (2020): 4056-4110 



\section{INTRODUCTION}

Already in 1920 Kossel gave an attractive explanation of the X-ray absorption phenomena $[1,2]$ :

.. in Röntgen spectra, we see that the electron cannot make small jumps outside the atom all the neighbour trajectories are already occupied by electrons so it must make a large jump to find a free trajectory, and the absorption occurs only when the frequency is high enough to make it reach the surface of the atom.

Starting from the sixties a cascade of studies have been published on theory, applications and techniques of x-ray methodologies, as is outlined in the review by Stumm von Bordwehr [2].

Exploring the X-ray absorption achievements from the point of view of the oxygen is particularly relevant given that oxygen is the third most important element in the universe [3]. Oxygen and its compounds are widespread and common. A large number of oxygen compounds are found in nature, and the earth's crust contains large amounts of oxygen- containing compounds. Oxygen itself is necessary for the life processes involving all forms of life. Many of the rocks and minerals are complex oxygen- containing compounds in the form of silicates, phosphates, and carbonates.

In short oxygen and its compounds are used in quantities that are almost beyond comprehension [4].

In this thesis the oxygen $\mathrm{K}$ edge $\mathrm{x}$-ray absorption spectra is discussed as an element specific analytical tool, where the oxygen is core electron is excited to the lowest empty states. The is core electron can be excited by the absorption of a photon in X-ray absorption (XAS), by the scattering of an electron in electron energy loss spectroscopy (EELS) or by the inelastic x-ray scattering (IXS) of a high energy photon. In this thesis is reported a systematical description of the oxygen $\mathrm{K}$ edges of atoms, molecules, ions, adsorbates, liquids and solids and the main experimental and theoretical aspects related to the measurements and the calculation of the oxygen $\mathrm{K}$ edge spectra. Furthermore we investigate in details two popular approaches 
to the modelling of $\mathrm{K}$ edges in molecular and solid systems: the coupled cluster hierarchy of methods and the DFT-based $\triangle \mathrm{SCF}$.

Nomenclature: The names of many spectroscopy techniques show some historical variation and also some research fields have different names for $\mathrm{x}$-ray absorption spectroscopy.

Throughout this thesis the term "oxygen $\mathrm{K}$ edge $\mathrm{x}$-ray absorption spectroscopy" will be used instead of "K edge". One can use the is orbital notation and some research areas use the term "X-ray absorption near edge structure" (XANES) or "near edge X-ray absorption fine structure"(NEXAFS). In this thesis, the abbreviation for x-ray absorption spectroscopy is XAS. The electron energy loss spectra is indicated with EELS and alternative notations include "near edge energy-loss spectroscopy" (ELNES). Finally inelastic $x-$ ray scattering (IXS) is alternatively indicated with "X-ray Raman scattering" (XRS) or "non-resonant inelastic x-ray scattering"(NIXS).

\section{I O K EDGE XAS THEORY}

In oxygen $\mathrm{K}$ edge $\mathrm{x}$-ray absorption, an $\mathrm{x}$-ray with $530 \mathrm{eV}$ energy excites the initial state of the system to a final state, where the initial and final states are many-body states of the system(Figure 1 ).

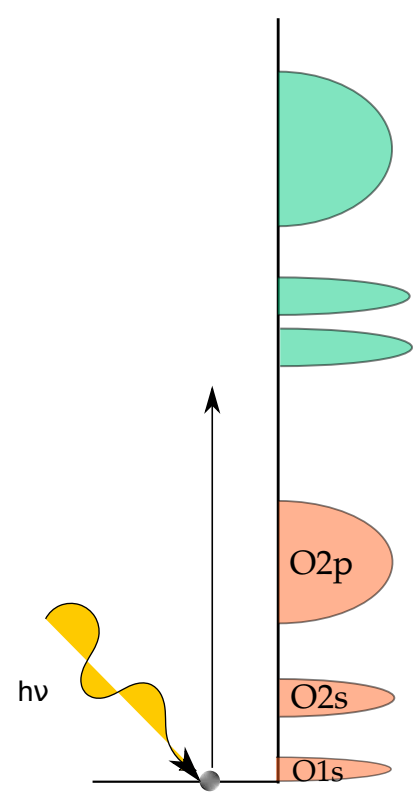

Figure 1: Pictorial representation of oxygen K edge absorption process. Where an electron from the is oxygen shell is excited to unoccupied states by $\mathrm{x}$-ray energy of $530 \mathrm{eV}$. 
The x-ray absorption process is calculated with the Fermi golden rule using the many body initial and final state wave functions $\Psi_{i}$ and $\Psi_{f}$ :

$$
\sigma(\omega) \approx \sum_{f}\left|\left\langle\Psi_{f}|\hat{\epsilon} \cdot R| \Psi_{i}\right\rangle\right|^{2} \delta\left(\omega-E_{f i}\right)
$$

$\hat{\epsilon} \cdot R$ is the transition operator that we have approximated as the dipole operator. $\delta$ indicates a $\delta$-function including $\mathrm{E}_{\mathrm{fi}}$, the energy difference between the states $\Psi_{i}$ and $\Psi_{f}$. Usually the Golden Rule is reduced to a one-electron approximation, and calculations are based on the dipole approximation. The goal is the computation of the dipole matrix elements and the transition energies where different approximations can be adopted.

\subsubsection{Density Functional Theory (DFT)}

\section{Independent particle approximation}

The main point of the independent particle approximation is the decoupling of the dynamics of the many body system in a single-particle description. The Hamiltonian can be rewritten with a term that mimics the interaction of the electrons with the average field that they feel and contains the kinetic energy of the electron and the potential energy of the electron in the field of the nuclei. With each independent electron is associated a spatial function and a corresponding energy eigenvalue, obtained by solving the Schrödinger equation with this Hamiltonian.

The independent particle approximation is also called the single particle approximation and the corresponding Hamiltonian the one-electron Hamiltonian.

Within the independent particle approximation it is possible to replace the many body quantities in the Fermi golden rule with single particle energies, since the excitation process is described by the promotion of an electron from an occupied to an unoccupied orbital (or singe particle state):

$$
M_{i \rightarrow f}=\left\langle\Psi_{f}|\hat{\epsilon} \cdot R| \Psi_{i}\right\rangle \approx\left\langle\psi_{f}|\hat{\epsilon} \cdot r| \psi_{i}\right\rangle
$$

The many body eigenstates and upper case operators on the left side of the equation are substituted by the lower case effective single particle states [5]. In the remainder of this thesis the term "single particle state" will be used as equivalent to term "orbital". 
We note that this change from the many body description to a single particle description involves two, coupled, approximations: (1) the description of the ground state in the independent particle approximation and (2) the description of the final state in the independent particle approximation. We note that in case of $2 p\left(\mathrm{~L}_{2,3}\right) \mathrm{x}$-ray absorption of transition metal system, it is the final state approximation that creates the main mistakes. But in case of oxygen $\mathrm{K}$ edges, we are dealing with a is core hole and the core hole can be accurately approximated with a potential.

Transition energies can be computed with the Delta Kohn Sham $(\Delta \mathrm{KS})$ method $[6,7]$. The idea behind the $\Delta \mathrm{KS}$ method is that the transition energies can be calculated as the difference between a ground state energy, computed with a self-consistent field calculation, and an ionized and excited state energy, obtained through a restricted open shell calculation. The procedure introduces an occupation number constrained in the is oxygen KS orbital, justified by its localized character. The non-orthogonal matrix elements between the Kohn-Sham ground state determinant and each excited state determinant are used in order to obtain the oscillator strength [8]. With the $\Delta \mathrm{KS}$ scheme two calculations are needed, one for the ground state and another one for the excited state.

Good agreement with experimental results have been obtained in the calculation of small molecules and metal adsorbate clusters oxygen $\mathrm{K}$ edges in terms of excitation energies [9] and trends in oscillator strengths [10] where the energy underestimation from the TD-DFT scheme is improved [1I].

Only one calculation is needed in an approximate procedure that is called the Slater transition state[12], even if different calculations are needed for each excited state. This procedure consists in the estimation of the excitation energy as orbital energy differences between two levels of variationally determined states, which are defined as the transition state, with only one half electron excited [12, 13].

The Slater transition state provides an estimation of the excitation energy with a correction to second order in the change of occupation. The formula describes a state where half an electron has been removed from the initial state $\psi_{i}$ and placed in the final state $\psi_{f}$. It is important to notice that the number of electrons is conserved. The ground state energy can be expressed as a Taylor expansion to the second order in the occupation numbers.

Once we have the two energies, the transition energy can be computed as a difference between them. Viewed as a balanced compromise between initial and final states, the transition-state orbitals can be used to represent both states, which reduce the evaluation of transition moments to single-electron 
transitions without affecting the other occupied orbitals [13]. The evaluation of the oscillator strengths becomes simplified since the same orbitals are used to describe the final and initial state. The weakness of the method is that one has to compute an x-ray absorption excitation for each state.

An approximation to the Slater transition state scheme that circumvents

a)

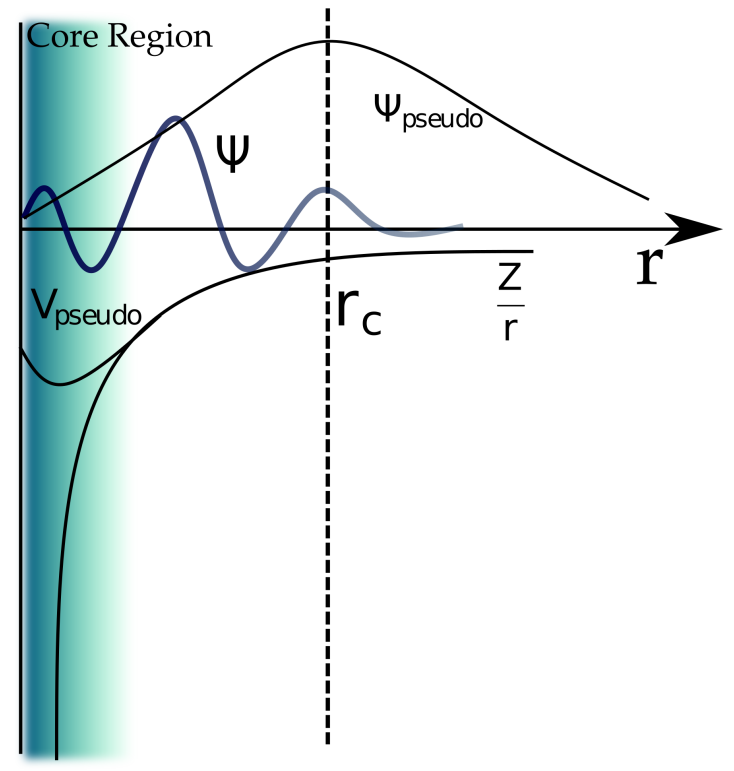

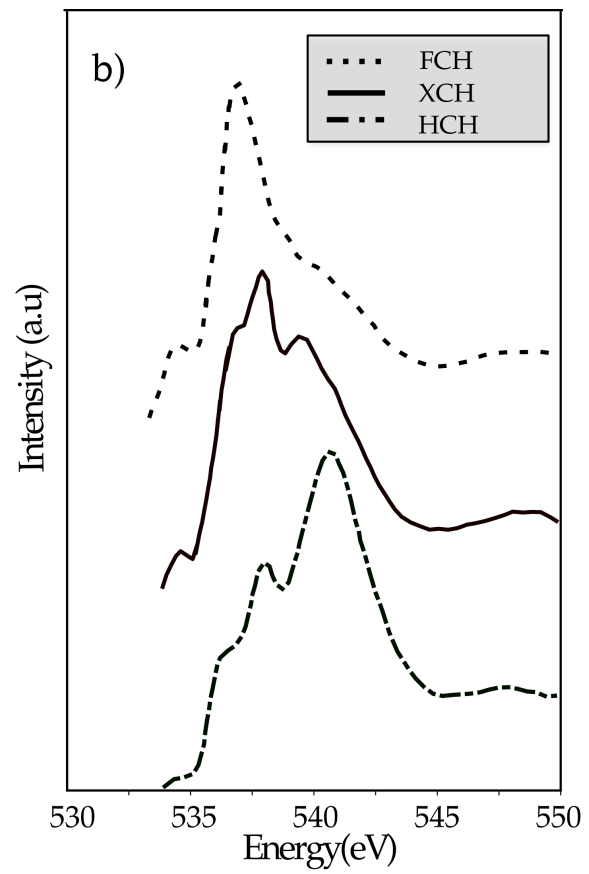

c)

$\mathrm{ECH}$

Virtual

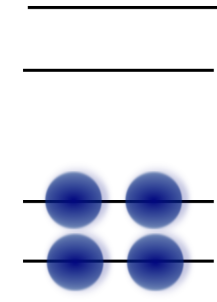

$\mathrm{Z}+1$

Core

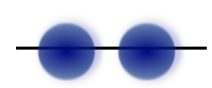

Z
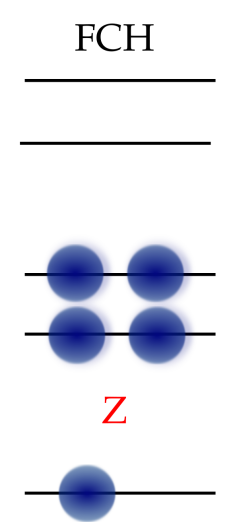

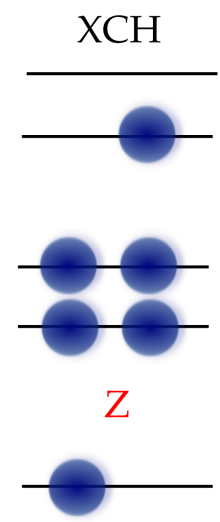

TS

$\mathrm{HCH}$
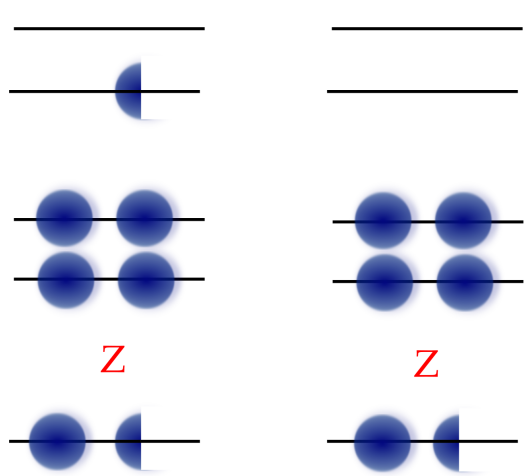

$\mathrm{Z}$

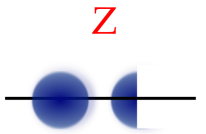

Figure 2: a)Representation of pseudowavefunction and pseudopotential. The pseudowavefunctions are smoother than the real wavefunction near the nuclei (where $r_{c}$ is the core radius), so the number of plane waves required is reduced. b) The $\mathrm{FCH}, \mathrm{XCH}$ and $\mathrm{HCH}$ approximations applied to liquid $\mathrm{H}_{2} \mathrm{O}$. [14] c)XAS final-state approximations: $\mathrm{Z}+1$ equivalent core hole, full core hole, excited core hole, Slater transition state transition state approximation [15]

the state-by-state calculations required is the transition potential approach formulated by Triguero et al. [16]. The approximation consists in removing half an electron from the oxygen is core level and to obtain all the possible 
excited states with only one global diagonalization from the potential generated by the core with the half occupied core-hole.

The complete spectrum evaluation in transition potential density functional theory (TP-DFT) consists in the calculation of the matrix elements between the orbitals corresponding to the $1 \mathrm{~s}$ and the unoccupied orbitals since the initial and the final states have the same orthogonal basis set [13]. It has been demonstrated that excitation energies computed in the TP-DFT framework require relaxation effects to be taken into account up to the second order [12]. Since the TP-DFT calculations can be considered as approximation to the Slater transition state scheme, the use of the half core-hole is theoretically justified. Extensions of the TP-DFT method, particularly suited when excitonic effects are strong, are the full core hole $(\mathrm{FCH})\left[{ }_{17}\right]$ and the excited core hole (XCH) [18].A correct description of the core hole is one of the challenging tasks of XAS simulations.

In Figure 2 some of the approximation schemes for the core hole are indicated. The Z+1 approximation, also called the empty core hole (ECH) approximation, introduces the core hole as an additional nuclear charge. The equivalent core hole approximation is justified since for electrons in the external shells, a deep core hole is felt as a positive charge [19, 20].

On an oxygen is core excited spectrum, oxygen is replaced by fluoride but because of the high electronegativity of the latter a charge transfer could be induced in the neighbouring atoms with a subsequent increase in the p-population of the excited core causing a distortion in the spectral shape [21]. Its applicability can be questioned also if the system has shallow core holes [22]. In the excited core hole $(\mathrm{XCH})$ approach, the potential of the first fully core excited state determines all the excited state orbitals. A strong approximation is made in the $\mathrm{XCH}$ procedure since the orbitals computed for one specific final state are supposed to describe the initial and the final states and no information about the initial ground states is present in the orbitals used $[13,18]$. The FCH method is similar to the $\mathrm{XCH}$ : the core electron is ionized which creates a hole in the core level but in this case, the impact of the excited electron on the core hole is neglected [21]. The DFT based methods cited above can, in principle, be applied to atoms, molecules and condensed phases. All these one electron calculations approximate the particle hole and the correlation effects. 


\subsubsection{DFT in solids}

If one deals with solids the Fermi Golden Rule is usually rewritten as:

$$
\sigma(\omega) \approx M^{2} \rho
$$

where $M$ is the one electron transition matrix element, and $\rho$ is the empty density of states. All electronic structure schemes can in principle be used in order to generate the density of states (DOS) and within a conceptual DFT framework, many routes can be used to evaluate the empty DOS and the XAS spectral shape.

The specific methods can be divided into:

- real space multiple scattering methods (FEFF [23]): the multiple scattering paths of the incoming photoelectron wave are computed solving the Green function equation in order to obtain the XAS spectrum.

- reciprocal space band structure methods (Wien2K [24], Quantumespresso [25]): the XAS spectrum is constructed from the energy of the photoelectron wave in different points of the band structure reciprocal space.

- real space wave function method (ADF [26], ORCA [27]) where the non-periodicity constraint makes the method suited for clusters and molecules.

We refer to the websites and original references of the methods indicated for details. The majority of solid state x-ray absorption calculations have been performed with local-density approximation of the density functional theory. A large number of specific procedures have been developed to solve the one electron Schrödinger equation. Using a linear method, different routines can be used to solve the band structure problem.

The two main schemes make both use of the variational principle for the one electron Hamiltonian and their difference lies in the formulation of the trial functions: linear combinations of energy independent augmented plane waves (LAPW) or muffin-tin orbitals (LMTO). The strength of these procedures is the use of the variational principle to solve the Schrödinger equation with energy independent basis functions, so that the secular equations become linear in energy [28]. A plane wave basis set with a pseudopotential approximation has been introduced to model the interaction between ions and valence electrons [29]. The pseudopotential neglects the inner core electrons and their strong potential due to the attraction to the nuclei. All electron descriptions of the system involve a strong orthogonal constraint between all the core and valence orbitals. The orthogonality relation presumes 
strong oscillations of the valence orbitals in the vicinity of the nucleus, as indicated in Figure 2 These oscillations, which require a large number of plane waves to be described, can be neglected in the pseudopotential framework, where the orthogonality relation is no longer valid and only a reduced number of plane waves is needed to describe the correct behaviour of the valence wave functions. The pseudopotential is then constructed for an initial DFT calculation for an isolated atom: a core radius $r_{c}$ should then be chosen and the valence wave functions are adapted to it in order to remove the nodal structure, these are the pseudo wave functions.

The pseudopotential approximation has been widely employed to model the oxygen $\mathrm{K}$ edge XAS in many different systems and satisfactory agreement with experiment has been reached.

\subsubsection{Core hole effects in solids}

It has been demonstrated that the core hole effects are stronger for spectra arising from elements in a ionic or covalent compound with lower electronegativity [30]. In Figure 3 the theoretical results for the ionic compound $\mathrm{BeO}$ are compared for both the metal and the oxygen edge with and without the core hole. The presence of the core hole alters both spectra, but in the oxygen case the modifications to the spectral shape are less significant. Although core hole effects have to be taken into account in order to have a good agreement with experiment, omitting any treatment of the core hole results usually in a shift of the threshold to higher energies and modified peak intensities in the low energy part of the spectra [30]. In Figure 3 the oxygen $\mathrm{K}$ edge spectra of $\mathrm{GeO}_{2}$ with and without the core hole is compared to the experiment. In this case, the relative intensities of some peaks are better reproduced without the inclusion of the core hole. This is evidence that the core hole potential, in the adopted supercell approach, is too attractive $[31,32]$.

If one compares the spectra without the inclusion of the core hole with the ground state DOS calculations, one can show that in some systems a ground state calculation can give features consistent with experiment [31] and provide information to explain the features in terms of orbital hybridization [33]. 

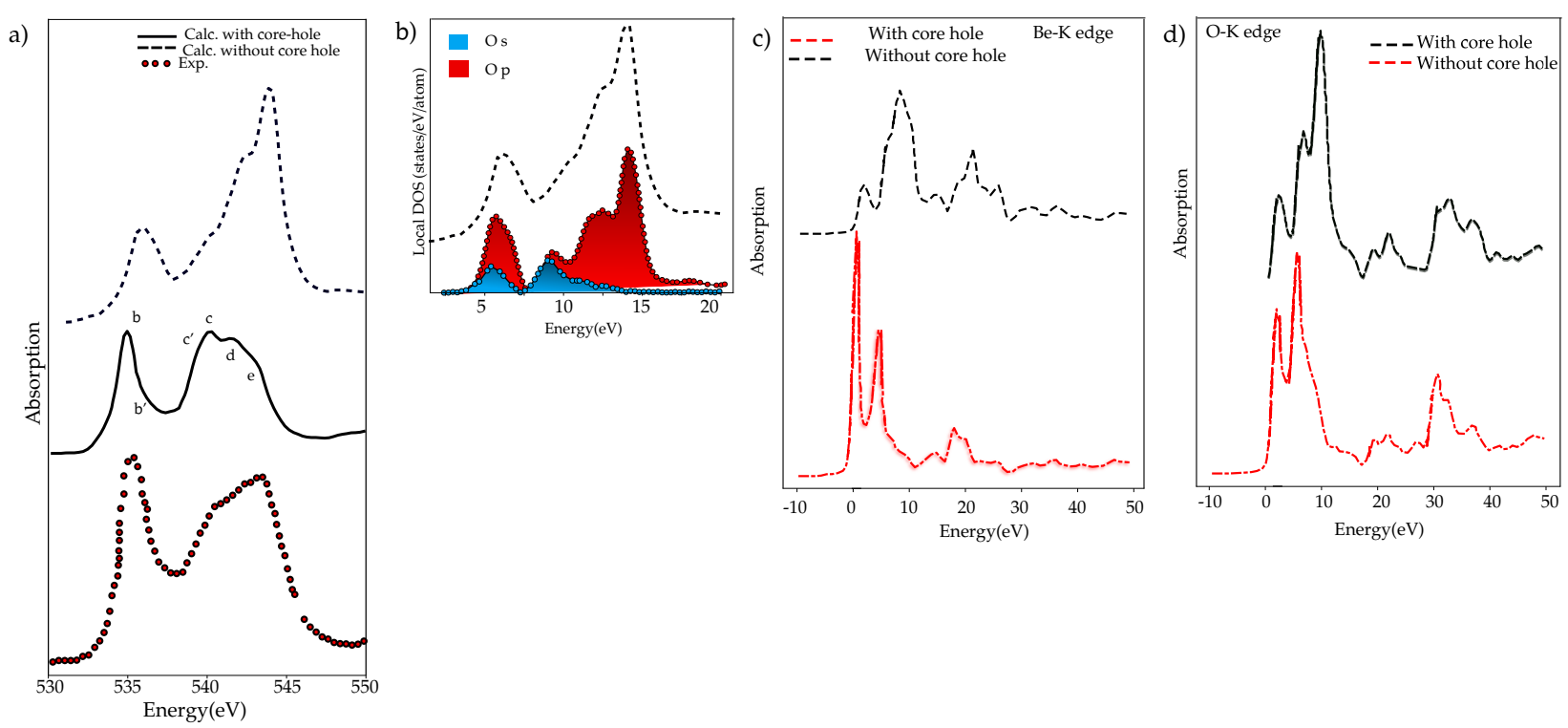

Figure 3: a) Theoretical $\mathrm{GeO}_{2}$ oxygen $\mathrm{K}$ edge with and without core hole compared with experiment $b$ ) Theoretical oxygen $\mathrm{K}$ edge XAS without core hole compared with a ground state oxygen DOS calculation [31]. c) The beryllium $\mathrm{K}$ edge of $\mathrm{BeO}$ calculated with and without core hole, d) The oxygen $\mathrm{K}$ edge of $\mathrm{BeO}$ calculated with and without core hole [30].

\subsubsection{Beyond the one particle approximation}

We have seen that the one body DFT calculations successfully describe the oxygen $\mathrm{K}$ edges of delocalized systems. However they lack accuracy in the description of partly localized and atomic edges, where many body and multiplet effects are important. This encouraged the push to go beyond the single particle picture with the inclusion of many body effects. After the first attempts made by Zangwill and Soven [34] and Zaanen et al. [35] to overcome the single particle description, nowadays the most widely used scheme are the Bethe Salpeter equations (BSE) [36] and the time dependent density functional theory (TD-DFT) [37] based methods.

When Runge and Gross [38] proposed the TD-DFT scheme to compute spectra, it became very popular for molecules but its implementation for solid systems is more recent [39]. The TD-DFT description of the x-ray absorption cross section enriched with the core hole contribution is also called the adiabatic local-density approximation. In this framework, the exchange correlation kernel depends only on the density in the ground state $(t=0)$. The potential generated by the kernel is local in space and time, and it is exactly this feature that produces an unsatisfactory long-range behaviour in solids $[37,40]$. 
Another obstacle to the correct description of solids with TD-DFT is linked to the inability of the latter to reproduce bound excitations [39] that leads to failure of the core hole effect description. Many attempts [41-43] have been made in order to make TD-DFT suitable for the description of the photoabsorption process in extended system, since it would be desirable in terms of simplicity [37], that is two-point equations are required in TD-DFT instead of the four-point equations in BSE. The Bethe-Salpeter equation [44] represents the equation of motion of a particle-hole state, as for example a photo excited electron to the conduction band from the is oxygen orbital.

The BSE description of x-ray absorption includes single-particle terms that describe the quasi-particle energies of the core hole and the excited photoelectron, together with the interaction between them. The interaction between the electron-hole pair has two terms: the Coulomb interaction, which includes adiabatic screening of the core hole, and an unscreened exchange term [45]. BSE calculations are considerably heavy in terms of computational cost and usually they have been limited to systems of restricted dimension.

A BSE calculation consists of the following steps (i) determine the groundstate electronic structure, (ii) correct the quasiparticle energies by adding a GW self-energy, (iii) evaluate the screening response to the core-hole, and (iv) determine the excitation spectrum of the BSE Hamiltonian. Liang et al. reported the oxygen $\mathrm{K}$ edge spectra of several transition metal oxides obtained through the BSE procedure and they showed the accuracy of the method in predicting the excitonic character of some peaks. The reproduction of the correct excitonic features of the spectra, is insured in BSE by the screened Coulomb interaction $\mathrm{W}$, present in the interaction kernel, that acts on the electron hole matrices and introduces a long range coupling between the electron and the hole wave functions [46].

An overview of the calculation procedures is given in Figure 4

\subsubsection{Charge transfer multiplet calculations}

Van Elp and Tanaka calculate the oxygen K edge spectra of transition metal ions from a charge transfer multiplet model [47]. The charge transfer multiplet model has been successfully applied to the metal $\mathrm{L}_{2,3}$ edges (2p XAS) of transition metal ions [48], which makes it interesting to check its applicability to the oxygen $\mathrm{K}$ edges of the same systems. There are however a number of differences between the metal $\mathrm{L}_{2,3}$ edge and the oxygen $\mathrm{K}$ edge, in particular the metal $\mathrm{L}_{2,3}$ edge creates excitonic states that are dominated 


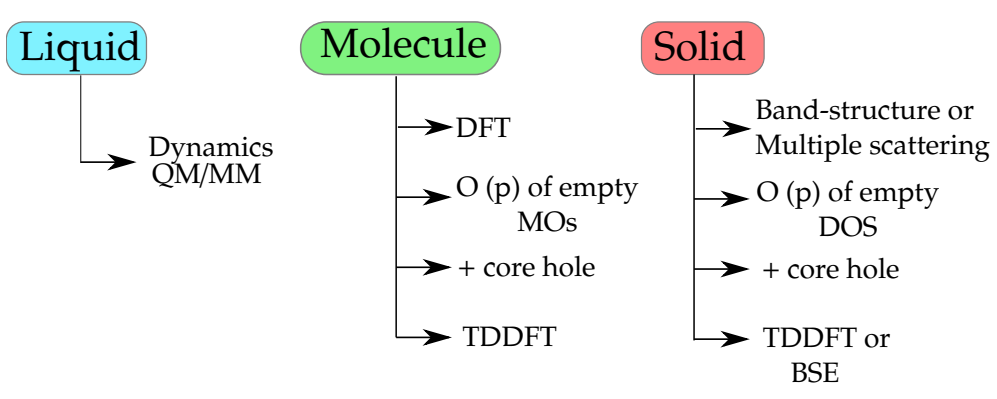

Figure 4: The oxygen $\mathrm{K}$ edge spectra can in first approximation be calculated from DFT codes, where molecules are usually calculated from molecular DFT codes and solids with band structure codes or multiple scattering. The oxygen p-contribution to the MO's or the oxygen p-projected DOS can be compared with the oxygen $\mathrm{K}$ edge spectral shape, where also matrix elements are included. As a next step, the core hole effect can be included, where many different procedures have been used both for molecules and solids. In case of solids one has to perform a super-cell calculation to avoid the core hole to interact with each other. Formally the correct way to calculate the oxygen $\mathrm{K}$ edges is to apply electron-hole excitation schemes such as TD-DFT or BSE, where the complexity of the calculations necessitate approximations. In systems where the dynamics of the system is important (including liquids) the calculations must be combined with molecular dynamics calculations. Because of the $300 \mathrm{meV}$ lifetime broadening, (in most cases) the effects of multiplets, orbital polarization and magnetic exchange are not visible. Vibrations are visible in case of (small) molecules. 
by large $2 p 3 d$ interactions (multiplet effects) and a large $2 p$ core hole spin orbit coupling. These interactions are absent for the oxygen $\mathrm{K}$ edge and only the dd-electron correlations remain. The exchange interaction of the dd-electrons is treated in spin-polarised DFT calculations, leaving out only the orbital polarization.

In other words, the charge transfer multiplet calculations include the orbital polarization, but they do not include the band structure effects due to the crystal structure. Because the oxygen $\mathrm{K}$ edge is broadened by the $300 \mathrm{meV}$ lifetime broadening, we do not expect that the orbital polarization effects will be visible.

\subsubsection{Post Hartree-Fock Methods}

Excitations from core orbitals to empty molecular orbitals cannot be computed with Hartree Fock because of some serious difficulties [49]:

- Convergence achievement,

- Variational collapse [50],

- Correlation effects,

Post Hartree Fock schemes have been investigated in order to overcome these difficulties. Approaches based on the coupled cluster hierarchy have been developed by Coriani [51] and Nooijen [52] using a linear response approach and an equation of motion respectively. These methods yield a very good description of the initial and final state wave functions but, because of the high computational cost, their applicability is limited to small molecular systems.

Asmuruf and Besley, proposed a second order perturbative approximation to the coupled cluster with single and double excitation (CCSD) based on a single configuration interaction (CIS). The methodology provides results equivalent to TD-DFT for the valence excitations but, because of the inclusion of the exact Hartree Fock exchange, better performance is obtained in the core excitation treatment [53]. Additional methods include the symmetry adapted cluster configuration interaction (SAC-CI) [54] and multiconfigurational self consistent field schemes [55].

The applicability of these methods is limited to small system since the choice of a relevant active space including local and non-local orbitals yields large calculations [56]. 
The oxygen $\mathrm{K}$ edge can be measured with $\mathrm{x}$-ray absorption spectroscopy (XAS), using a $530 \mathrm{eV}$ x-ray for the excitation of a 1 s core electron to an empty state. There are two alternative techniques based on the inelastic scattering of electrons, respectively the inelastic scattering of photons. Electron energy loss spectroscopy (EELS) measures the energy loss of an electron and inelastic x-ray scattering the energy-loss of an x-ray beam. An x-ray absorption experiment needs:

- An x-ray source

- A monochromator

- A detector

where the sample condition is also an important aspect. In the next section we briefly introduce some aspects of the experimental conditions.

\subsubsection{Sources for $x$-ray absorption spectroscopy}

Historically, XAS was measured with x-ray tubes [2]. X-ray tubes give the largest part of their $x$-ray emission in the form of intense monochromatic $\mathrm{x}$-ray energies related to the specific core-core $\mathrm{x}$-ray emission channel, for example the aluminium $\mathrm{K} \alpha$ source at $1486 \mathrm{eV}$.

A second source of $x$-rays from the tube is the so called bremsstrahlung. The bremsstrahlung energy loss is due to the slowdown of electrons from the metal in the anode and gives rise to a continuous spectrum of $\mathrm{x}$-rays. Bremsstrahlung is much lower in intensity than the specific $x$-ray emission lines but with a source optimised for bremsstrahlung emission, one has enough photons for good x-ray absorption measurements. The 1920 paper from Kossel describes the analysis of the XAS spectral shape [1]. Kossel already realised that the $\mathrm{x}$-ray absorption spectral shape was influenced by the valence electrons and the other "external conditions" of the absorbing atom in the surroundings where it was embedded. Using an x-ray tube, Fischer measured a series of oxygen $\mathrm{K}$ edges in 1971 with $0.9 \mathrm{eV}$ resolution [57].

To compare x-ray sources one usually compares the spectral brightness or brilliance of the $x$-ray beam, which is defined as the number of photons (in a certain bandwidth) per second and per $\mathrm{mm}^{2}$ and $\mathrm{mrad}^{2}$, where the brilliance of an X-ray tube is $10^{8}$. Depending on the details of the XAS experiment (for example is one measures a gas or a solid), the divergence and 
the spot size is often not crucial and the x-ray flux is a more important property. The flux is defined as the number of photons (in a certain bandwidth) per second.

Synchrotron radiation emerged in the 1960's but a limiting factor for oxygen $\mathrm{K}$ edge XAS measurements was the absence of $\mathrm{x}$-ray monochromators for the 500 to $600 \mathrm{eV}$ range with good resolution. Synchrotron radiation covers all x-ray energies with a brilliance between $10^{13}$ to $10^{18}$ photons $/ \mathrm{sec}$ $/ \mathrm{mm}^{2} / \mathrm{mrad}^{2}$, in other words $10^{5}$ to $10^{10}$ more bright than an $\mathrm{x}$-ray tube. The x-ray monochromators were improved in the 1970's and in 1980 Stohr et al. measured the oxygen $\mathrm{K}$ edge spectrum with $2 \mathrm{eV}$ resolution [58]. A big step in experimental resolution was set in the 1980's with the development of the SX700 monochromator by Petersen [59] and the DRAGON monochromator by Chen and Sette [6o]. Both beamlines reached a resolution that was better than the oxygen $\mathrm{K}$ edge lifetime broadening of $0.3 \mathrm{eV}$, which implies that from this time the experimental resolution of oxygen $\mathrm{K}$ edges was not limited by the experimental resolution, but by the intrinsic lifetime broadening.

More than $95 \%$ of all oxygen $\mathrm{K}$ edges are measured with synchrotron radiation sources, but next to the synchrotron and x-ray tubes a number of additional x-ray sources exist, respectively:

- plasma sources

- high harmonic generation (HHG)

- x-ray free electron lasers (XFEL)

With a plasma source one is also able to measure soft x-ray spectra [61]. Kuhl et al. [62] measured an oxygen $\mathrm{K}$ edge spectrum with $2 \mathrm{eV}$ resolution. Soft $\mathrm{x}-$ rays can also be generated with HHG laser systems. Most present HHG laser systems operate up to $100 \mathrm{eV}$ and they are especially used for femtosecond pump-probe experiments. Extensions to the oxygen $\mathrm{K}$ edge at $530 \mathrm{eV}$ are in principle possible on some newly developed sources and/or they are foreseen in the near future [63-67]. The use of HHG and XFEL lasers for time-resolved experiments is discussed in the section 2.6. The oxygen $\mathrm{K}$ edge of cosmic sources is measured with x-ray satellites XMM Newton and Chandra, with a resolution of approximately $2.0 \mathrm{eV}$ [68]. 


\subsubsection{Detection techniques}

Transmission detection: X-ray transmission is the most direct technique to measure an oxygen $\mathrm{K}$ edge XAS spectrum and it allows the quantitative detection of the $x$-ray absorption cross section. The attenuation of $530 \mathrm{eV}$ $\mathrm{x}$-rays is only $100 \mathrm{~nm}$ at the oxygen $\mathrm{K}$ edge of solid oxides, implying that one needs thin samples. A transmission measurement is only quantitatively correct if the measured sample is homogeneous in thickness. If thickness variations occur, the spectral shape will appear distorted with essentially the high-intensity peaks appearing flattened. This so-called pin-hole effect also plays a role for samples that are inhomogeneous, for example samples containing nanoparticles or otherwise nano-structured objects that are measured with an area-averaged $x$-ray probe.

Alternative detection methods are based on decay channels of the core hole. Fluorescence yield (FY) measures the amount of $x$-rays that are emitted. The energy that is gained by the electron that fills the core hole can also be used to emit another electron in the Auger process. In the further relaxation of the core hole more electrons can be emitted and electron yield (EY) also measures the total amount of emitted electrons.

Fluorescence Yield detection: The core hole is filled with an electron from another shell and the energy can be emitted as an x-ray.

Detecting the fluorescent $x$-rays after radiative core hole decay can yield a method that is proportional to the XAS spectral shape. In total fluorescence yield (TFY) detection one detects all emitted x-ray photons, from all elements present in the sample. Assuming that all non-resonant photoionization provide a constant background, the TFY measures the XAS spectral shape. However, spectral distortions can occur due to saturation and selfabsorption effects, because usually the x-ray emission (being off-resonance) has a larger probing depth than the penetration depth of the x-ray at the edge [69-71]. While scanning through an absorption edge the $x$-ray penetration depth varies due to the XAS spectrum and if there is no background absorption, the observed spectral shape would be highly distorted. If the background $\mathrm{x}$-ray absorption dominates, the $\mathrm{x}$-ray penetration depth is constant over the edge and no saturation occurs. In other words, TFY can only be used for materials where the observed element is dilute.

We note that if the concentration of the absorbing element is very low, the FY from the other elements might dominate the signal and the specific FY from the dilute element will have low signal-to-noise. Bulk oxides are too concentrated and their TFY spectra appear highly saturated. In addition to 
saturation effects, there can be effects due to self-absorption, in other words the re-absorption of the emitted x-ray emission. Again this effect is only significant for concentrated samples [72]. Saturation and self-absorption effects are angle dependent, which also offers a method to quantify the saturation effects.

In case of the oxygen $\mathrm{K}$ edge, there are no spectral modifications due to state dependent decay [73-75]. Instead of TFY only a limited energy range of the x-ray emission can be detected. Such partial fluorescence yield (PFY) method can have an advantage if the $\mathrm{x}$-ray emission signal is dominated by another element. As such, PFY measurements allow the detection of XAS spectra of very low concentrations, down to the $100 \mathrm{ppm}$ range [76]. A special type of PFY is inverse PFY (IPFY). In this method one detects the FY of a different element than the element from which the XAS spectrum is measured, where one can prove that in IPFY measurements no saturation effects can occur [77].

Electron Yield detection: In total electron yield (TEY) electrons that escape from the sample are detected in electron analysers. This can include the integrated signal from energy-dispersive detectors or, in case of conductive samples, the current measurement to the sample. If the energy of the electrons is selected, one speaks of partial electron yield (PEY) or with the detection of a specific decay channel also of Auger electron yield [78].

A special property of electron yield is that the electrons have an escape depth that is in the order of a few $\mathrm{nm}$, much shorter than the $\mathrm{x}$-ray penetration depth, implying that in most cases the TEY signal is not saturated [79, 80]. Thus electron yield detection turns XAS into a surface-sensitive probe. This can be used to enhance the signal from the surface and near surface part of the sample. The surface sensitivity also implies that the measured signal is not exactly equal to the bulk signal. In case of surface oxidation or surface modification, the electron yield signal is different from the XAS spectrum of the bulk system. Even for a perfect surface in a single crystal the TEY signal will be different from the bulk because the atoms in the top layer at the surface have a different surroundings and corresponding electronic structure. Due to the probing depth of only $4 \mathrm{~nm}$, the top layer contributes significantly ( $10 \%$ ) to the total spectral shape. In addition, most crystal show surface reconstructions that extend a few layers into the crystal. This makes that TEY measurements will usually be affected by surfaceinduced effects to some extend. If TEY is measured under a gas atmosphere, conversion electron yield (CEY) appears as an additional option [81]. In a gas atmosphere, the electrons emitted from the sample create ions that are 
counted and as such the CEY method effectively measures the XAS spectral shape. Often two detectors are used that measure respectively the gas phase and the sample surface plus gas phase, allowing both signals to be differentiated [82]. The conversion of electrons to ions can be dependent on the electron kinetic energy and the molecules in the gas phase, which implies that CEY should be well calibrated during the experiments.

Another type of CEY is applied in liquids. One can measure the XAS spectrum of a surface that is in contact with a liquid, by detecting the ions that are generated in the liquid by the escaping electrons, also known as the ioncurrent detection [83]. In general these CEY methods should always be well calibrated and tested to check their linearity with the XAS cross section.

Ion Yield detection: Ion yield detection is different from the ions measured in CEY methods described above. In ion yield one measures a specific ion with a mass spectrometer while scanning through the XAS spectrum. Himpsel et al. used ion yield detection to measure the surface of a $\mathrm{CaF}_{2}$ crystal [84]. Ion yield detection is a common technique in gas phase experiments, where it is linked to photofragmentation detection. The oxygen $\mathrm{K}$ edge can be measured by detecting various possible ionic fragments that are produced by the XAS process [85].

Ion yield detection has been applied to the oxygen $\mathrm{K}$ edge by Hayakawa et al. who measured a series of cerium oxide clusters [86]. Figure $5 b$ gives an overview of the detection techniques of $\mathrm{x}$-ray absorption spectroscopy, including transmission, electron yield, fluorescence yield and ion yield methods.

\subsubsection{Inelastic X-ray scattering}

Inelastic X-ray scattering, also known as X-ray Raman Scattering (XRS), measures the inelastic scattering of hard x-rays, not resonant with a core level. IXS is a low-intensity experiment, implying that an intense synchrotron or XFEL beamline is required.

The oxygen $\mathrm{K}$ edge can be measured with IXS by detecting the $530 \mathrm{eV}$ energy loss. By changing the scattering angle of the IXS experiment one can modify the momentum transfer. An important application of q-dependent measurements is the change of the ratio between dipole and quadrupole transitions. Pylkanen et al. applied q-dependent IXS to the oxygen $\mathrm{K}$ edge of a series of alcohols [87]. The advantage of the hard x-rays used in IXS is that one can measure the oxygen $\mathrm{K}$ edge under extreme conditions, for example high-pressure [88]. High-pressure studies include the study of the 


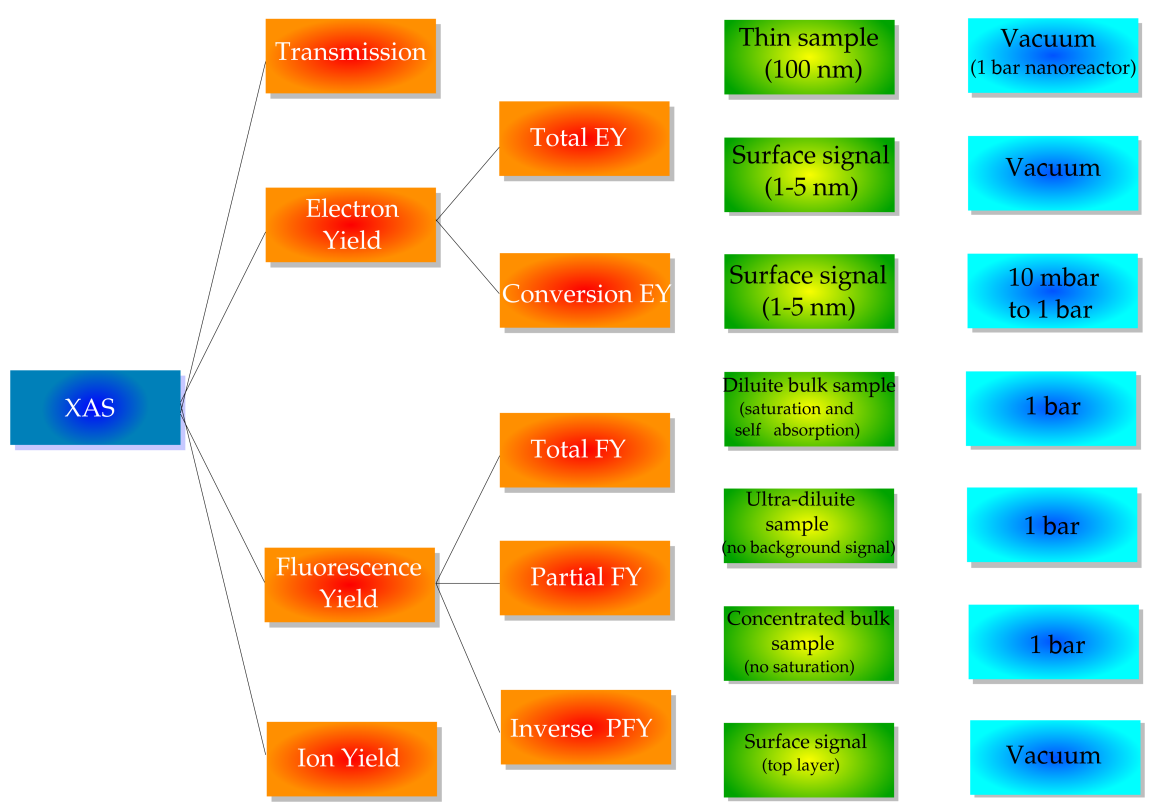

(a) Detection techniques of XAS

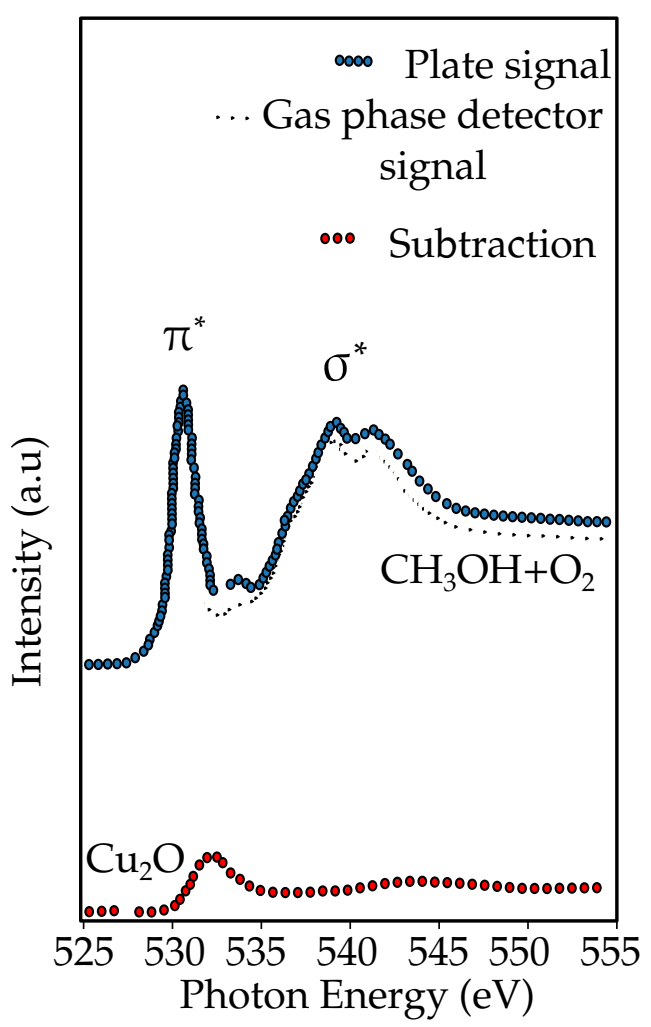

(b) In situ

Figure 5: Upper panel: overview of the detection techniques of $\mathrm{x}$-ray absorption spectroscopy, including transmission, electron yield, fluorescence yield and ion yield methods. Sample measurement details are given in green and the pressure in blue. Lower panel:the oxygen $\mathrm{K}$ edge of a mixture of methanol and oxygen at 0.52 mbar that reacts on a $\mathrm{Cu}$ foil at $520 \mathrm{~K}$. The detector near the surface detects the signal from the gas phase and the surface; the detector far from the surface detects the pure gas phase spectrum[82]. 
phases of supercritical and solid phases of $\mathrm{H}_{2} \mathrm{O}$ [89] and studies regarding the nature of the high-pressure phases of silicates [90].

\subsubsection{Electron energy loss spectroscopy}

Electron energy loss spectroscopy (EELS) is usually measured with transmission electron microscopes (TEM), but historically some dedicated EELS machines have also been used, for example the EELS experiments performed by the group of Fink [91]. The resolution of EELS in dedicated electron microscopes is $0.3 \mathrm{eV}$ or better since the 1990s [92].

As a rule EELS is performed with an electron beam with an energy above $100 \mathrm{keV}$. The high energy and low momentum transfer effectively turns the 1 /e Coulomb operator in inelastic electron scattering into an effective optical operator. Studies using low-energy ( $100 \mathrm{eV}$ ) electrons, for example resonant EELS at electron energies equal to core level binding energies is mainly limited to metal edges and to valence excitations $[93,94]$. Using a $100 \mathrm{keV}$ electron beam at low scattering angle, one can approximate the EELS spectrum with the dipole approximation. Under these approximations (high primary energy, low scattering angle) EELS becomes essentially equivalent to x-ray absorption. In EELS one can tune the dipole/quadrupole ratio by changing the scattering angle. In the remainder of the manuscript we will treat EELS spectra conceptually equal to XAS spectra. TEM-EELS has the advantage that one can reach atomic resolution, allowing the measurement of an oxygen K edge of one atom (or better one atomic column) through a sample.

\subsubsection{In situ experiments}

Oxygen $\mathrm{K}$ edge XAS is usually performed in vacuum. Soft $\mathrm{x}$-rays have a penetration depth through air in the order of $1 \mathrm{~cm}$. In principle this is long enough to perform transmission or x-ray fluorescence experiments. From 1988 Fischer et al. performed a series of in-situ TFY experiments at pressures up to 100 mbar [95]. Using nanoreactors in a scanning transmission x-ray microscopy (STXM) the oxygen $\mathrm{K}$ edge of $\mathrm{Fe}_{3} \mathrm{O}_{4}$ supported on $\mathrm{SiO}_{2}$ has been studied at 1 bar under Fischer-Tropsch conditions [96]. A more popular approach is to use electron yield detection. Because TEY is surface sensitive and TFY is bulk sensitive their comparison can be very useful. TFY and TEY were compared to study the changes of propylene on the surface of $\mathrm{Ag} / \mathrm{Y}$ zeolites. The use of both TFY and TEY nicely separates the behaviour of the surface and the bulk [97].

Traditionally in-situ TEY measurements are performed in combination with 
$\mathrm{x}$-ray photoemission and the XPS detector makes it difficult to go above 10 mbar pressure. A series of 1 to 10 mbar in-situ oxygen $\mathrm{K}$ edge studies have been performed on so-called near-ambient pressure photoemission beamlines [98]. This allows the study of the surface of heterogeneous catalysts under working conditions in a flow-through reactor.

Figure $5 \mathrm{~b}$ shows the oxygen $\mathrm{K}$ edge of a mixture of methanol and oxygen at 0.52 mbar that reacts on a $\mathrm{Cu}$ foil at $520 \mathrm{~K}$. The spectral shapes are a combination of $\mathrm{O}_{2}, \mathrm{CH}_{3} \mathrm{OH}$ and the $\mathrm{Cu}_{2} \mathrm{O}$ surface species. The system uses one detector that measures the gas phase and a second detector that measures the combination of the surface and the gas phase. Using the difference between the two detectors, the surface signal can be revealed. Experiments can be performed at different gas mixtures, pressures and temperatures, as has been described in a number of applications [98-102]. A series of detailed in-situ oxygen $\mathrm{K}$ edge studies has been applied to vanadium oxides, under different gas atmospheres and with different loadings of the vanadium. The analysis of the oxygen K edge, in combination with DFT studies allows the distinction between separate vanadium, silica and interface contributions [103, 104].

A more recent development is to use CEY detection up to pressures of 3 bar [105].

\subsubsection{Time-resolved XAS}

One can combine oxygen $\mathrm{K}$ edge XAS with time resolved measurements. Cavalleri et al measured the time evolution of the oxygen $\mathrm{K}$ edge of $\mathrm{VO}_{2}$ using a laser-sliced synchrotron beam with a time-resolution of $500 \mathrm{fs}$, from which new information on the photoinduced metal-insulator transition in $\mathrm{VO}_{2}$ was found [106].

The LCLS X-ray FEL was used to study the changes in the excited-state electronic structure of the nucleobase thymine, by using the changes in the oxygen K edge [107]. A number of fs oxygen K edge XFEL studies have been performed on adsorbates, for example $\mathrm{CO}$ on $\mathrm{Ru}($ O001) [108, 109]. Femtosecond XFEL studies focussing on the oxygen is x-ray emission channel have also been performed on water [110, 111]. As mentioned above, HHG sources have reached the oxygen $\mathrm{K}$ edge energy and femto- and attosecond oxygen $\mathrm{K}$ edge spectra are foreseen in the near future [63-67]. 


\subsubsection{X-ray and electron spectromicroscopy}

Before discussing some $\mathrm{X}$-ray microscopy options, first transmission electron microscopes (TEM) will be briefly discussed. With TEM one can measure core levels below $1000 \mathrm{eV}$ with atomic resolution (down to $0.1 \mathrm{~nm}$ ) [112, 113], for example Muller et al studied the $\mathrm{Si}: \mathrm{SiO}_{2}$ interface, indicating a different electronic structure of oxygen at the interface [114]. Egoavil et al. studied $\mathrm{La}_{2} \mathrm{CoMnO}_{6}$ films on $\mathrm{SrTiO}_{3}$ (111) substrates [115]. They use cobalt and manganese EELS to determine the site specific valence, in combination with the oxygen $\mathrm{K}$ edge based EELS maps. Zhou et al. studied $\mathrm{ZrO}_{2}-(\mathrm{La}, \mathrm{Sr}) \mathrm{MnO}_{3}$ thin films using the atomic EELS contrast of several elements, including the oxygen $\mathrm{K}$ edge [116]. The methods used to derive the atomic resolution images are explained by Wang et al. [117], who used the oxygen K edge of $\mathrm{SrTiO}_{3}$ as an example. With scanning transmission x-ray microscopy one can measure the oxygen K edge with spatial resolution. Such STXM-XAS experiments are analogous to STEM-EELS, with the difference being that with TEM one can reach $0.1 \mathrm{~nm}$ resolution and with TXM $10 \mathrm{~nm}$. On the other hand STXM allows for thicker samples and operando conditions [118]. De Smit et al. used the oxygen K edge STXM data to provide a thickness profile of their $\mathrm{Fe}_{3} \mathrm{O}_{4} / \mathrm{SiO}_{2}$ catalyst sample, while the metal edges were used to probe their spatial variation and valence [96]. Sharma et al. studied the oxygen $\mathrm{K}$ edge spectra in graphene and graphene oxide with a STXM microscope [119]. The STXM microscopes can also be used to select a specific small oxide material and, for example, Ward et al. studied the oxygen $\mathrm{K}$ edge of uranium minerals [120], while a series of rare earth oxides have also been studied with a STXM [121].

An alternative $\mathrm{x}$-ray microscopic technique is $\mathrm{x}$-ray photoelectron emission microscopy (PEEM). In X-ray PEEM one measures the emitted electron with an electron microscopic lens system. X-ray PEEM is mainly used to study magnetic structure, using either magnetic linear dichroism (MLD) or magnetic circular dichroism (MCD). Kinoshita et al. studied the magnetic domain structure of a $\mathrm{NiO}(100)$, comparing the $x$-ray PEEM images measured at the oxygen $\mathrm{K}$ edge with the nickel L edge [122]. Oxygen K edge PEEM was also used to study polymer mixtures [123].

\subsubsection{Using the oxygen K edge as resonance}

Resonance studies include the use of the oxygen K edge in resonant photoemission spectroscopy (RPES) and resonant inelastic x-ray scattering (RIXS). In addition one could perform $\mathrm{x}$-ray scattering at the oxygen $\mathrm{K}$ edge and res- 
onant EELS with electron sources. The oxygen K edge of solid samples does not contain excitonic states, which turns the two-dimensional RIXS plane essentially in a convolution of the oxygen $K$ edge and the non-resonant 1s2p $\mathrm{x}$-ray emission spectrum [124]. The oxygen $\mathrm{K}$ edge is particularly used as a resonator for the iridates. Because the Ir $2 p$ edge lies in the hard $x$-ray range, it has low-resolution. The soft $\mathrm{x}$-ray oxygen $\mathrm{K}$ edge then adds improved resolution to study the electronic structure. This has been used for $\mathrm{Ba}_{2} \mathrm{IrO}_{4}$ [125] and $\mathrm{SrIrO}_{4}$ [126], where the magnon dispersion is measured. The oxygen K edge RIXS spectra are also used to study the core hole clock mechanism, in which it is reasoned that by changing the excitation energy, one change the ratio between resonant and normal decay channels. An example is the study on the oxygen K edge RIXS of alcohols [127]. 


\section{Part II}

\section{ATOMIC AND MOLECULAR SYSTEMS}

In part II the discussion moves to oxygen atoms, ions, binary molecules, water and larger molecules containing oxygen, including biomolecular systems. The results are disccused in term of chemical environment influence on the photoadsorption process and available decay channels giving rise to the spectra.

Chapter 3 reports the performance of a core-valence separated scheme within the coupled cluster (CC) hierarchy of methods CC singles (CCS), CC singles and approximate doubles (CC2) and CC singles and doubles (CCSD) in reproducing the K-edge x-ray absorption spectra of the low- $\mathrm{Z}$ elements carbon, oxygen and fluorine in formaldehyde $\left(\mathrm{CH}_{2} \mathrm{O}\right)$, carbonyl fluoride $\left(\mathrm{CF}_{2} \mathrm{O}\right)$, formyl fluoride $(\mathrm{CHFO})$ and formic acid $(\mathrm{CHOOH})$. Moreover, a simulation of the vibronic progressions in the $1 \mathrm{~s} \rightarrow \pi^{*}$ bands of both carbon and oxygen in formaldehyde at the CVS-CCSD level is reported. Part II is based on the papers: Frati F., M. O.J.Y. Hunault, and F.M.F. de Groot. "Oxygen K-edge X-ray Absorption Spectra." Chemical Reviews 120.9 (2020): 4056-4110 and F. Frati, F.M.F. De Groot, J. Cerezo, F. Santoro, L. Cheng, R. Faber and S. Coriani Coupled cluster study of the $x$-ray absorption spectra of formaldehyde derivatives at the oxygen, carbon, and fluorine K-edges. The Journal of Chemical Physics, 151(6)(2019) 064107. 



\section{ATOMIC AND MOLECULAR SYSTEMS}

\section{I THE OXYGEN ATOM}

Atomic oxygen has a total of eight electrons with the electronic configuration $1 s^{2} 2 s^{2} 2 p^{4}$.

The $2 p$ orbitals are partially filled and there are different ways to arrange the electrons in the $2 p$ orbitals. Figure 6 shows the oxygen K edge of atomic oxygen on a logarithmic scale. In the first peak at $528 \mathrm{eV}$, a is electron fills one of the two $2 p$ holes and the final state configuration is $1 s^{1} 2 s^{2} 2 p^{5}$. This configuration has two open shells, i.e. $1 s^{1} 2 p^{5}$. The term symbols of such configuration can be found from the multiplication of the term symbols from the individual shells, in this case ${ }^{2} \mathrm{~S}$ times ${ }^{2} \mathrm{P}$, yielding respectively ${ }^{1} \mathrm{P}$ and ${ }^{3} \mathrm{P}$. In oxygen these states are split by $2.5 \mathrm{eV}$. Including the $2 \mathrm{p}$ spin-orbit coupling yields the ${ }^{3} \mathrm{P}_{0},{ }^{3} \mathrm{P}_{1}$ and ${ }^{3} \mathrm{P}_{2}$ states. This implies that from the ${ }^{3} \mathrm{P}_{2}$ Hunds rule ground state one can reach three final states, respectively the ${ }^{1} \mathrm{P}_{1},{ }^{3} \mathrm{P}_{1}$ and ${ }^{3} \mathrm{P}_{2}$ states. The ${ }^{3} \mathrm{P}_{1}$ and ${ }^{3} \mathrm{P}_{2}$ states are separated by only $40 \mathrm{meV}$ and appear as one peak in the experiment.

Energy states of open shell systems The description of the energy states of open shell systems needs a description of the coupling of their angular momenta to describe the many electron states. The $\mathrm{L}, \mathrm{S}$ and $\mathrm{J}$ quantum numbers of the multi-electron state are indicated with a so-called term symbol that is written as ${ }^{2 S+1} \mathrm{~L}_{\mathrm{J}}$. The $2 \mathrm{p}^{4}$ configuration of an oxygen atom has the term symbols ${ }^{3} \mathrm{P},{ }^{1} \mathrm{D}$ and ${ }^{1} \mathrm{~S}$, where the orbital quantum is indicated with letters:. $\mathrm{L}=\mathrm{O}$ is written as $\mathrm{S}$ (harp), $\mathrm{L}=1$ as $\mathrm{P}$ (rincipal), $\mathrm{L}=2$ as $\mathrm{D}$ (iffuse) and $\mathrm{L}=3$ as $\mathrm{F}$ (undamental).

The ${ }^{3} \mathrm{P}$ state is split by the $2 p$ spin-orbit coupling into respectively ${ }^{3} \mathrm{P}_{0},{ }^{3} \mathrm{P}_{1}$ and ${ }^{3} \mathrm{P}_{2}$ states, using the rule that $J$ runs from $|L-S|$ to $L+S$ in steps of one. The Hunds rules determine the state with the lowest energy, respectively the state with (1) the largest S, (2) the largest $\mathrm{L}$ and (3) the largest J, where in case a shell is less than half-filled the lowest J is the ground state due to the inverted effect of the spin-orbit coupling. This makes the ${ }^{3} \mathrm{P}$ state the ground state. The ${ }^{1} \mathrm{D}$ has an energy of $2.0 \mathrm{eV}$ above the ground state and the excitation energy of the ${ }^{1} \mathrm{~S}$ state is $5.5 \mathrm{eV}$. The $2 \mathrm{p}$ spin-orbit splitting is small and the energy difference between the ${ }^{3} \mathrm{P}_{2}$ ground state and the ${ }^{3} \mathrm{P}_{1}$ state is only $25 \mathrm{meV}$. 
The ${ }^{1} \mathrm{P}_{1}$ state, which is $2.5 \mathrm{eV}$ higher in energy is not visible due to its low relative intensity of $10^{-5}$. The result is that the excitation from the $1 \mathrm{~s}$ to the $2 p$ state has effectively one visible peak as shown in Figure 6 at $528 \mathrm{eV}$.

The ionization energy of the oxygen atom relates to the excitation of the

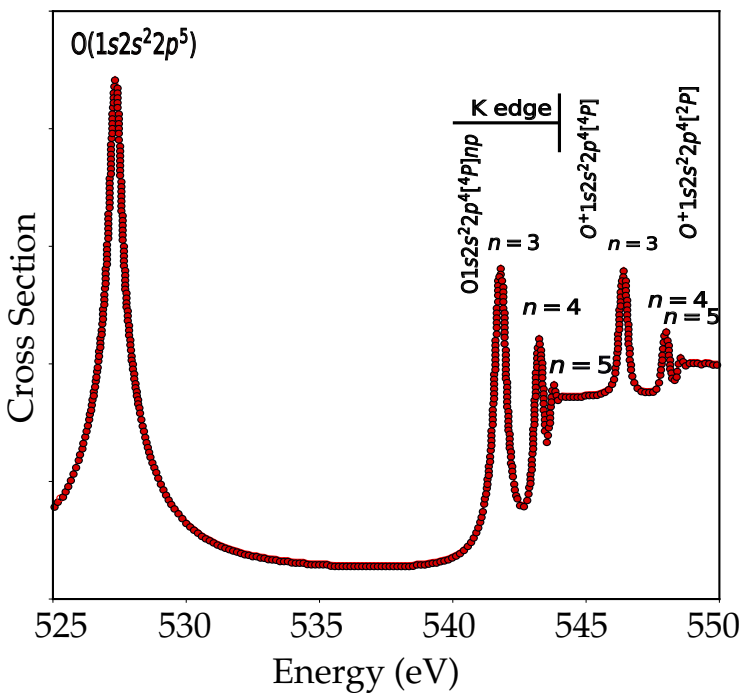

Figure 6: Experimental atomic oxygen $\mathrm{K}$ edge $\mathrm{x}$-ray absorption spectrum from ref. [128]. The 1s to $2 p$ transition is visible at $527 \mathrm{eV}$. The Rydberg states are visible above $540 \mathrm{eV}$.

is electron into a free electron. In fact there are two ionization energies depending on the relative orientations of the spins of the excited core electron and the two holes in the $2 p$ states. This yields respectively states with $S=2$ or $\mathrm{S}=4$, as indicated in Figure 6 at $545 \mathrm{eV}$ and $548 \mathrm{eV}$ [129].

The peaks in between the edge and the ionization energy above $540 \mathrm{eV}$ are assigned to transitions from the $2 p^{4}$ ground state to the $1 s^{1} 2 p^{4}(n p)$ states, where $n$ can take the values 3, 4, 5, etc., the so-called Rydberg states. The $1 s^{1} 2 p^{4}$ configuration is split into multiplets by the 1s2p exchange, the $2 p 2 p$ electron-electron interactions and the small $2 p$ spin-orbit coupling. The term symbols are respectively ${ }^{2} S,{ }^{2} \mathrm{P},{ }^{2} \mathrm{D}$ and ${ }^{4} \mathrm{P}$, from which only the ${ }^{2} \mathrm{P}$ and ${ }^{4} \mathrm{P}$ states gain detectable intensity. The ${ }^{2} \mathrm{P}$ and ${ }^{4} \mathrm{P}$ states are split by $3.5 \mathrm{eV}$ and this yields two series of Rydberg states as given numerically in table 1 [130].

The 1s2p peak and the Rydberg peaks in the oxygen $\mathrm{K}$ edge of the oxygen atom have been calculated by Petrini and Araujo [131]. The analysis of resonant Auger spectra gives a more complete understanding of the nature of the states in the oxygen K edge XAS [132]. 


\begin{tabular}{|c|c|c|c|c|}
\hline Configuration & $\begin{array}{c}\text { Energy } \\
\text { Expt. } \\
(\mathrm{eV})\end{array}$ & $\begin{array}{c}\text { Energy } \\
\text { Th. (eV) }\end{array}$ & $\begin{array}{c}\text { Relative } \\
\text { Inten- } \\
\text { sity } \\
\text { Expt. }\end{array}$ & $\begin{array}{c}\text { Relative } \\
\text { Inten- } \\
\text { sity } \\
\text { Th. }\end{array}$ \\
\hline $1 \mathrm{~s} 2 \mathrm{~s}^{2} 2 \mathrm{p}^{5}(3 \mathrm{P})$ & 527.8 & 528.1 & 100 & 100 \\
\hline $1 s 2 s^{2} 2 p^{4} 3 p(4 P)$ & $541 \cdot 3$ & 542.2 & 6.2 & 5.1 \\
\hline $1 \mathrm{~s} 2 \mathrm{~s}^{2} 2 \mathrm{p}^{4} 4 \mathrm{p}\left({ }^{4} \mathrm{P}\right)$ & 542.6 & 543.8 & 1.6 & 1.9 \\
\hline $1 \mathrm{~s} 2 \mathrm{~s}^{2} 2 \mathrm{p}^{4} 5 \mathrm{p}\left({ }^{4} \mathrm{P}\right)$ & 543.2 & $544 \cdot 3$ & 0.36 & 1.1 \\
\hline $1 \mathrm{~s} 2 \mathrm{~s}^{2} 2 \mathrm{p}^{4} 3 \mathrm{p}\left({ }^{2} \mathrm{P}\right)$ & 545.6 & 545.6 & $5 \cdot 4$ & 2.0 \\
\hline $1 \mathrm{~s} 2 \mathrm{~s}^{2} 2 \mathrm{p}^{4} 4 \mathrm{p}\left({ }^{2} \mathrm{P}\right)$ & $547 \cdot 2$ & $547 \cdot 2$ & 1.9 & 0.5 \\
\hline $1 \mathrm{~s} 2 \mathrm{~s}^{2} 2 \mathrm{p}^{4} 5 \mathrm{p}\left({ }^{2} \mathrm{P}\right)$ & $547 \cdot 7$ & 547.8 & 0.36 & 0.24 \\
\hline
\end{tabular}

Table 1: Comparison of experimental and theoretical energies and relative intensities of the two Rydberg series converging to the ${ }^{4} \mathrm{P}$ and ${ }^{2} \mathrm{P}$ limit of atomic oxygen [130].

\subsubsection{Oxygen ions}

When oxygen is ionized the ground state changes from $2 \mathrm{p}^{4}$ to $2 \mathrm{p}^{3}$, yielding a new ground state symmetry as indicated in table 3. Because the positive charge of the $\mathrm{O}^{1+}$ ion, it costs more energy to excite its 1 s core electron. The energy shift from a neutral oxygen atom to a $\mathrm{O}^{1+}$ ion is $4.5 \mathrm{eV}$. Table 2 makes use of data on oxygen ions from $x$-ray satellites such as the Chandra X-ray observatory and XMM Newton. These x-ray satellites are able to detect the oxygen K edge XAS spectrum with around $200 \mathrm{meV}$ resolution [129]. Oxygen is often studied as it plays a key role in the understanding of the chemical evolution of the Universe [133].

If the oxygen atom is ionised to $\mathrm{O}^{1+}$ ion, the oxygen 1 s binding energy increases due to the decrease of the valence electrons [134]. The $\mathrm{O}^{2+}$ and $\mathrm{O}^{3+}$ ions have a XAS spectrum containing three peaks. Due to their less-thanhalf-filled configuration and related Hunds rule ground state with minimum J, three final states have similar intensity.

Photoionization and photoabsorption cross sections used to model important astrophysical processes have been provided first by theory [135] as at that time limited experimental reliable data were available. Early theoretical photoionization cross sections are computed by means of HartreeSlater wave functions [136] and Dirac-Slater wave functions [137]. Pradhan et al. [134] calculated the resonant transitions energies and the resonance oscillator strengths from the ground state of different oxygen ions (of astrophysical importance) by means of Breit-Pauli R-matrix method. 


\begin{tabular}{cccc}
\hline Ion & Conf. & Symmetry & $1 \mathrm{~s} 2 \mathrm{p}(\mathrm{eV})$ \\
\hline $\mathrm{O}$ & $2 \mathrm{~s}^{2} 2 \mathrm{p}^{4}$ & ${ }^{3} \mathrm{P}$ & 528.8 \\
$\mathrm{O}^{1+}$ & $2 \mathrm{~s}^{2} 2 \mathrm{p}^{3}$ & ${ }^{4} \mathrm{~S}$ & 533.3 \\
$\mathrm{O}^{2+}$ & $2 \mathrm{~s}^{2} 2 \mathrm{p}^{2}$ & ${ }^{3} \mathrm{P}$ & 537.2 \\
$\mathrm{O}^{3+}$ & $2 \mathrm{~s}^{2} 2 \mathrm{p}^{1}$ & ${ }^{2} \mathrm{P}$ & 545.8 \\
$\mathrm{O}^{4+}$ & $2 \mathrm{~s}^{2}$ & ${ }^{1} \mathrm{~S}$ & 554.6 \\
$\mathrm{O}^{5+}$ & $2 \mathrm{~s}^{1}$ & ${ }^{2} \mathrm{~S}$ & 562.3
\end{tabular}

Table 2: Theoretical 1s2p excitation energies of oxygen ions. The Hunds rule ground state is given and the symmetries of the main peaks in the final state [134].

Photoabsorption cross sections have been calculated with the R-matrix approaches [138] and multiconfigurational methods (MCHF [139] and MDHF [132]). McLaughlin et al. [140] reported photoabsorption cross sections obtained with RMPS and high resolution experimental measurements.

\subsection{MOLECULAR SYSTEMS}

\subsubsection{The binary molecules $\mathrm{CO}, \mathrm{NO}$ and $\mathrm{O}_{2}$}

In $\mathrm{CO}, \mathrm{NO}$ and $\mathrm{O}_{2}$ molecules there are respectively 14,15 and 16 electrons that can be placed into the molecular orbitals. The orbitals in molecules can be constructed from the combination of the atomic orbitals. Figure 7 shows the molecular orbitals of $\mathrm{O}_{2}, \mathrm{CO}$ and NO . The is orbitals of $\mathrm{O}_{2}$ are not overlapping with the neighbour orbitals and keep their atomic character. The $2 \mathrm{~s}$ and $2 \mathrm{p}$ orbitals of $\mathrm{O}_{2}$ have overlap with the neighbour $2 \mathrm{~s}$ and $2 \mathrm{p}$ orbitals and have $\sigma$ bonding and anti-bonding orbitals. The $2 p$ orbitals of $\mathrm{O}_{2}$ also have $\pi$ overlap with the neighbour $2 \mathrm{p}$ orbitals. The anti-bonding $\pi_{2 p}^{*}$ molecular orbitals contain two paired electrons and the anti-bonding $\sigma_{2 p}^{*}$ are empty.

At higher energies one can find the molecular orbitals due to $3 \mathrm{~s}, 3 \mathrm{p}$ and 3d atomic orbitals. The molecular orbitals of $\mathrm{NO}$ are equivalent with one electron in the anti-bonding orbital $\pi_{2 p}^{*}$ and $\mathrm{CO}$ has zero electrons in the anti-bonding orbital $\pi_{2 p}^{*}$. Because for $\mathrm{CO}, \mathrm{NO}$ and $\mathrm{O}_{2}$ molecules there are two partly empty 2 p-states, one expects two peaks in the XAS spectrum corresponding respectively to transitions to the $\pi^{*}$ and $\sigma^{*}$ states.

$\mathbf{O}_{2}$ : The $\pi_{2 p}^{*}$ orbital in $\mathrm{O}_{2}$ is doubly degenerate and half filled, implying that different electronic states can be obtained depending on the electronic 


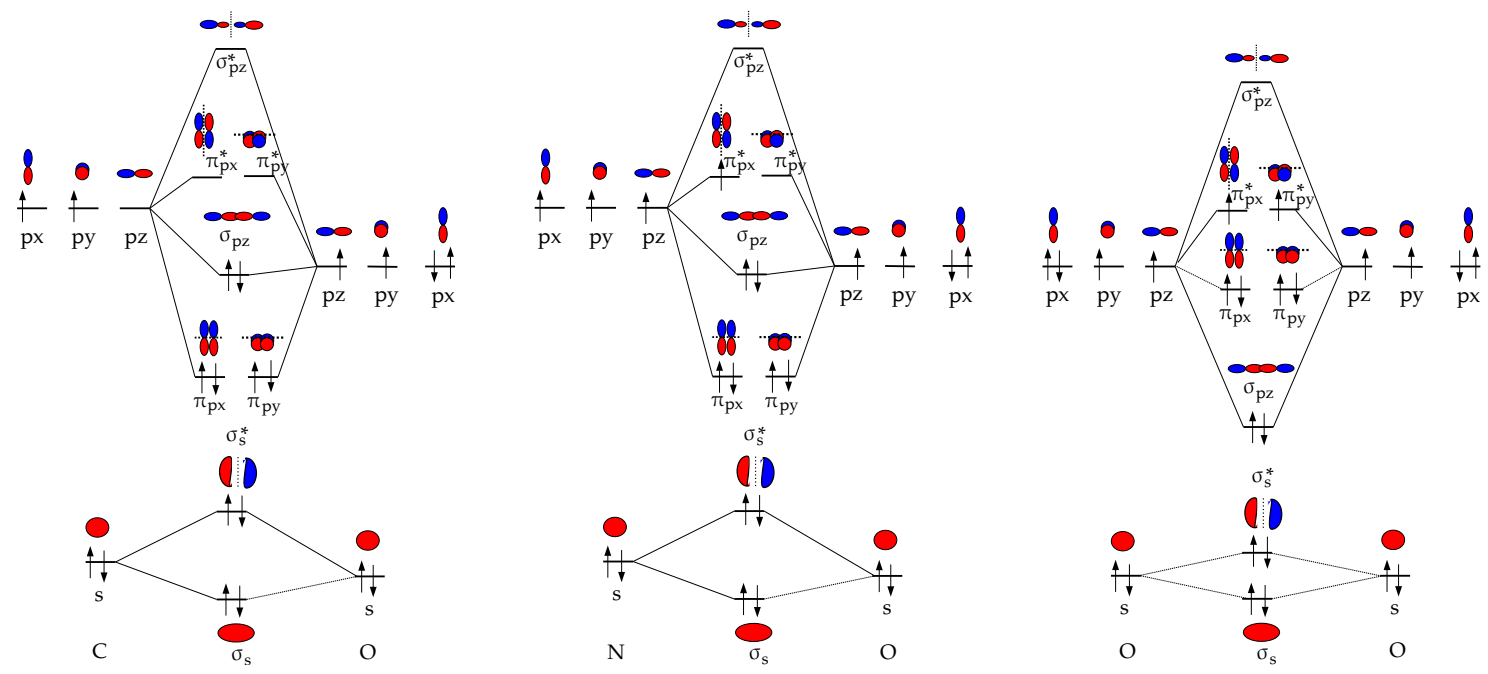

Figure 7: Molecular orbital diagram of $\mathrm{CO}, \mathrm{NO}$ and $\mathrm{O}_{2}$. The three $\mathrm{MO}$ energy schemes are equivalent, where in case of $\mathrm{O}_{2}$ the order of the bonding $\sigma_{2 p}$ and $\pi_{2 p}$ states are inverted due to a decreased influence of the $2 \mathrm{~s}$ states. The anti-bonding orbital $\pi_{2 p}^{*}$ is filled with respectively 0,1 and 2 electrons, creating the triplet ground state for $\mathrm{O}_{2}$.

configuration.

There are three different states, respectively ${ }^{3} \Sigma_{g},{ }^{1} \Delta_{g}$ and ${ }^{1} \Sigma_{g}$ corresponding to different arrangement of these electrons in the $C_{2}$ point group symmetry. The ground state of molecular oxygen is the triplet state ${ }^{3} \Sigma_{g}$.

The oxygen $\mathrm{K}$ edge spectrum of $\mathrm{O}_{2}$ has been extensively studied [141-154]. The strong first resonance in the oxygen $\mathrm{K}$ edge absorption spectrum $\left(\mathrm{E}_{\text {edge }}\right)$ at $531 \mathrm{eV}$ (O 1s $\longrightarrow \pi^{*}$ ) has been analysed and the vibrational structure has been resolved [153].

The ionization energy $\mathrm{E}_{\text {ion }}$ of $\mathrm{O}_{2}$ is found at approximately $547 \mathrm{eV}$ and like for the oxygen atom, it is split into a quartet and a doublet state depending on the coupling of the core electron spin to the valence spins, indicated in Figure 8.

The challenging part of the assignment of the peaks in the $\mathrm{O}_{2}$ absorption spectrum is the region between 535 and $545 \mathrm{eV}$. In this region we expect the $2 p \sigma^{*}$ peaks but also the Rydberg states.

The $1 \mathrm{~s} \longrightarrow \sigma^{*}$ resonance is split into two features converging to the ${ }^{4} \Sigma^{-}$and ${ }^{2} \Sigma^{-}$ionization thresholds at 543.39 and $544.43 \mathrm{eV}$ [145, 152, 154, 156]. Kosugi et al. reported symmetry resolved oxygen $\mathrm{K}$ edge spectra where two $\sigma$ features with different intensities are assigned to the bound state transitions $2 p \sigma^{*}[141,157]$. The solid state oxygen $\mathrm{K}$ edge spectra of $\mathrm{O}_{2}$ show no strong quenching or broadening of these peaks [158]. This suggest that the peaks cannot be assigned to Rydberg states, because the Rydberg states will 

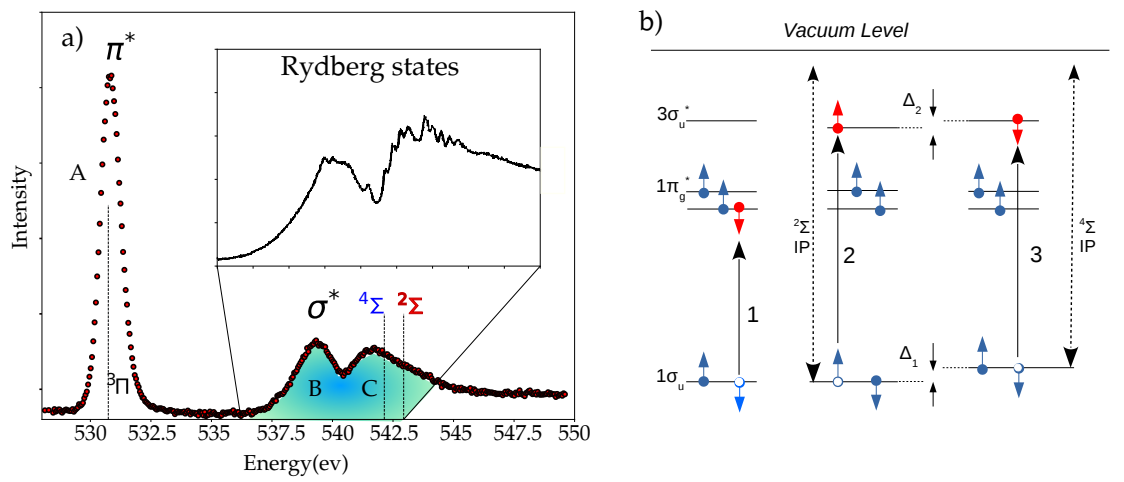

Figure 8: a) The oxygen $\mathrm{K}$ edge of $\mathrm{O}_{2}$ from ref. [141], including a high-resolution inset of the Rydberg states. b) Energy level diagram and allowed transitions, including the ionization potential for spin up and down indicated as ${ }^{2} \Sigma$ and ${ }^{4} \Sigma$. The exchange splitting arises from differences in the magnetic exchange interactions in the final states yielding the experimental peaks $B$ and C [155].

behave differently in the solid state, due to strong intermolecular overlap of the extended $3 \mathrm{p}$ and $4 \mathrm{p}$ states.

Wurth at al. [155] reported an exchange splitting of $4 \mathrm{eV}$ and assigned the $\sigma^{*}$ resonance to the spin up and spin down transitions $1 \sigma_{g} \longrightarrow 3 \sigma_{\mathfrak{u}}^{*}$ [155].

Ruckman et al. studied the $\mathrm{O}_{2}$ gas phase spectrum in comparison with the oxygen $\mathrm{K}$ edge of the alkali metal superoxides and they reported an experimental exchange splitting value of $0.4 \mathrm{eV}$ [159], with both components located in peak B. The exchange splitting value that can be found in the literature span a broad range and this can be ascribed to the theoretical difficulties in treating the Coulomb and exchange interaction in the core hole excited states [160]. These two different interpretations of the absorption interaction lead to different conclusions.

Comparing the adsorbed $\mathrm{O}_{2}$ and gas phase, the changes in the peak positions and intensities are interpreted either as quenching of the exchange splitting [155] or as the consequence of the Rydberg nature of peak $C$ in free $\mathrm{O}_{2}$ [159]. The analysis of the de-excitation spectra helps the separation between $\sigma^{*}$ and Rydberg core excited states. The de-excitation spectra excited at feature $\mathrm{C}$ can be understood from the decay of one or more Rydberg states whereas the de-excitation spectra for the chosen excitation energies within feature $B$ are associated with the decay of the $2 p \sigma^{*}$ state.

Under pressure $\mathrm{O}_{2}$ condenses to a liquid and at $5.5 \mathrm{GPa}$ it solidifies to a solid. As a function of pressure several solid $\mathrm{O}_{2}$ phases exist [161]. The oxygen $\mathrm{K}$ edges of the liquid and solid $\mathrm{O}_{2}$ phases show that as a function of pressure the $\pi^{*}$ and the $\sigma^{*}$ peaks move to higher energy, consistent with a 
decreasing $\mathrm{O}-\mathrm{O}$ interatomic distance. In addition the $\pi^{*}$ loses intensity due to the increased interaction between the $\mathrm{O}_{2}$ molecules leading to a singlet ground state for the $\epsilon\left(\mathrm{O}_{2}\right)_{4}$ phase [161].

NO: The electronic configuration of $\mathrm{NO}$ is equivalent to that of $\mathrm{O}_{2}$ with only one electron in the $2 p_{\pi}$ orbital, resulting in a ground state symmetry with term symbol ${ }^{2} \Pi$. Figure 9 shows the oxygen $\mathrm{K}$ edge spectrum of NO, which is similar to that of $\mathrm{O}_{2}$. The $\pi^{*}$ peak at $532.55 \mathrm{eV}$ followed by the $\sigma^{*}$ peak at $546 \mathrm{eV}$. Because NO is an open shell molecule the excitation of the inner $1 s_{\sigma}$ electron in the oxygen $\mathrm{K}$ edge results in the coupling between the unpaired electron spins of the core and valence shells [162]. This implies that the $\pi^{*}$ peak at $532.7 \mathrm{eV}$ is split into three states, respectively ${ }^{2} \Sigma^{-},{ }^{2} \Delta$, ${ }^{2} \Sigma^{+}[141,162]$.

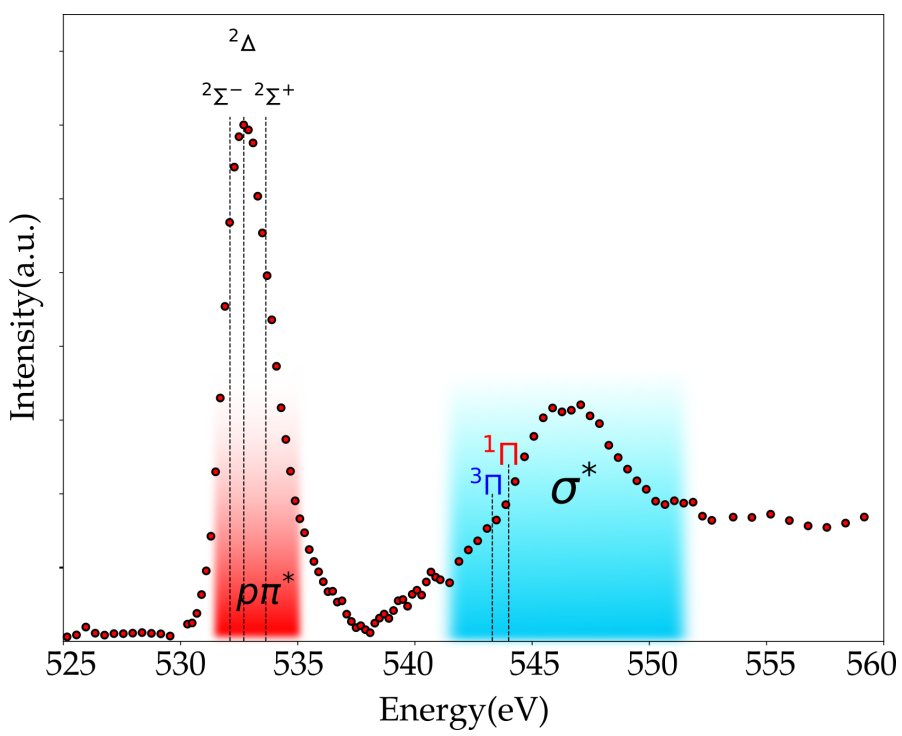

Figure 9: Oxygen $\mathrm{K}$ edge spectrum of $\mathrm{NO}$ where the ionization limits are indicated as ${ }^{1} \Pi$ and ${ }^{3} \Pi[163]$. The three theoretical components $\left({ }^{2} \Sigma^{-},{ }^{2} \Delta\right.$ and $\left.{ }^{2} \Sigma^{+}\right)$ in 1s2p peak at $532.7 \mathrm{eV}$ are indicated.

The core-valence interactions also yield two ionization thresholds $(\Delta=$ $\mp 1 / 2)^{3} \Pi$ and ${ }^{1} \Pi[164]$. The symmetry resolved spectra confirm their boundstate transition character.

The atomic population analysis [165] and photoelectron measurements [166] find that the $2 \mathrm{p} \pi^{*}$ orbitals have an high percentage of nitrogen character. Auger decay investigations suggest that the three doublets state give the highest contribution to the high energy auto-ionizing lines observed in the oxygen K Auger spectrum [167]. The feature with a maximum around 540 
$\mathrm{eV}$ has been assigned to Rydberg transitions with quantum number $\mathrm{n}=3$ while the $\sigma$ resonances are observed above the ionization threshold [141, 162]. Three series (ns, np and nd) of Rydberg states are found [164].

CO: Carbon monoxide ( $\mathrm{CO})$ has a singlet ground state, where there are no electrons in anti-bonding orbitals. The molecule has a closed shell configuration and its ground state is a totally symmetric singlet ${ }^{1} \Sigma^{+}$. In Figure 10 , the $\mathrm{CO}$ oxygen $\mathrm{K}$ edge spectra is reported. The first feature in the oxygen $\mathrm{K}$ edge is the bound excitation into an antibonding $\pi^{*}$ orbital that can be vibrationally resolved [153, 164]. Also for $\mathrm{CO}$ the $\sigma$ shape resonance appears above the ionization threshold. The two higher energy peaks are assigned to $3 \mathrm{~s} \sigma$ and $3 \mathrm{p} \pi$ Rydberg excitations.

The features in the continuum have been interpreted as the $1=3$ shape resonance enhancement in the $\sigma$ channel [168] or as a transition to the quasibound $\sigma^{*}$ [169]. In Table 3 we compare the excitation energies to the $2 p \pi^{*}$

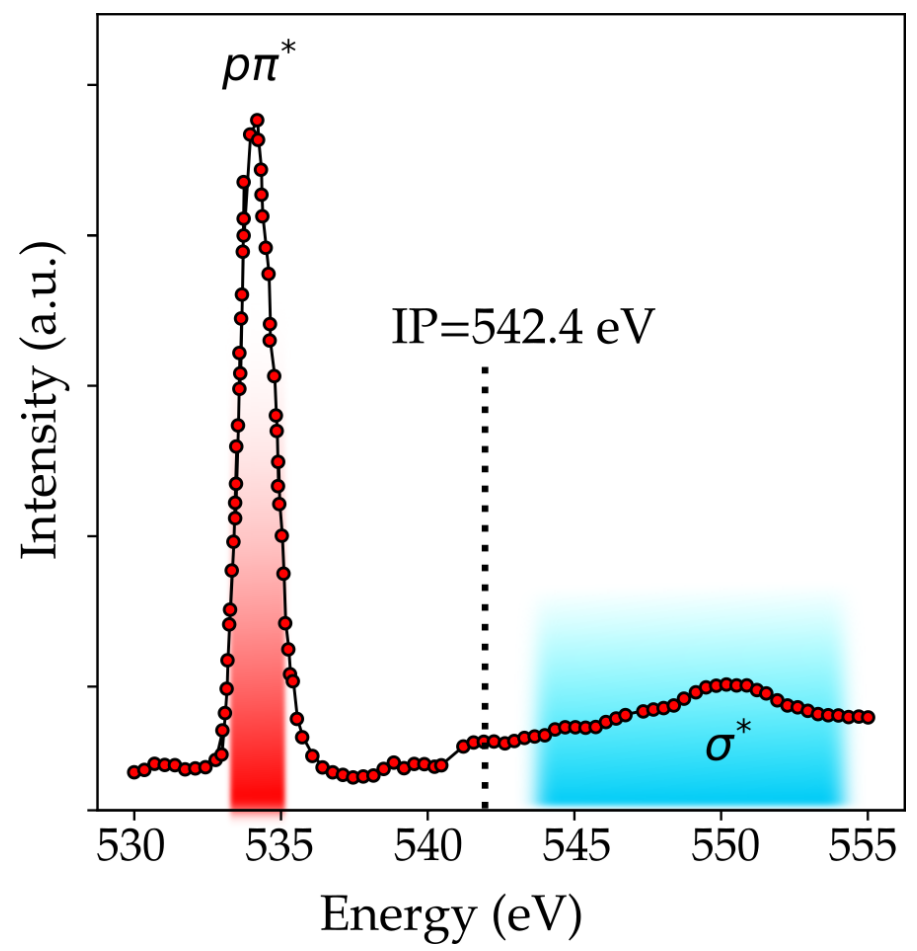

Figure 10: Oxygen $\mathrm{K}$ edge spectrum of $\mathrm{CO}$ where the ionization potential (IP) is indicated [157].

peak in $\mathrm{CO}, \mathrm{NO}$ and $\mathrm{O}_{2}$. The excitation energy increases from $\mathrm{O}_{2}$ to $\mathrm{CO}$, indicating that it becomes more difficult to excite the is core electron to the $2 \mathrm{p} \pi^{*}$ state. The nitrogen $\mathrm{K}$ edge shift down in going from $\mathrm{N}_{2}$ to NO. An increase in the excitation energy was also seen for the change from oxygen atoms to ions. Extrapolating this to NO, indicates that the oxygen atom in 
Table 3: The energy position of the $2 \mathrm{p} \pi^{*}$ peak of binary molecules and ions.

\begin{tabular}{c|cc}
\hline \multicolumn{3}{c}{ The $2 \mathrm{p} \pi^{*}$ peak energy } \\
\hline & Energy $(\mathrm{eV})$ & reference \\
\hline $\mathrm{O}_{2}^{-}$ & 529.0 & {$[159]$} \\
$\mathrm{O}_{2}$ & 530.8 & {$[141]$} \\
$\mathrm{O}_{2}^{+}$ & 533.2 & {$[170]$} \\
$\mathrm{NO}$ & 532.7 & {$[141]$} \\
$\mathrm{CO}$ & 534.2 & {$[153]$} \\
$\mathrm{CO}^{+}$ & 533.8 & {$[171]$} \\
\hline
\end{tabular}

$\mathrm{NO}$ is more positive than in $\mathrm{O}_{2}$, with the $\mathrm{N}$ atom being more negative. In $\mathrm{CO}$ the oxygen atom is even more positive. Ions of two atom oxides: Lindblad measured the oxygen $\mathrm{K}$ edge of a $\mathrm{O}_{2}^{+}$and $\mathrm{CO}^{+}$molecules. The $2 \mathrm{p} \pi^{*}$ peak shifts to higher energy in $\mathrm{O}_{2}$, in agreement with its positive charge implying a higher excitation energy [170].

Ruckman et al. measured the oxygen $\mathrm{K}$ edge spectra of the alkali superoxide $\mathrm{KO}_{2}$ [159]. These systems can be considered as containing $\mathrm{K}^{+}$and $\mathrm{O}_{2}^{-}$ions and the oxygen $\mathrm{K}$ edge of $\mathrm{KO}_{2}$ can be interpreted as that of $\mathrm{O}_{2}^{-}$. The $2 \mathrm{p} \pi^{*}$ peak shifts to lower energy in $\mathrm{O}_{2}^{-}$. The removal of a valence electron in the CO molecule will create a vacancy in the $5 \sigma$ orbital, implying that the first peak corresponds to transitions into this orbital.

The peak at $533.8 \mathrm{eV}$ is the $2 \mathrm{p} \pi^{*}$ peak that has shifted to lower energy from $534.2 \mathrm{eV}$ in $\mathrm{CO}[171]$, with the corresponding carbon $2 \mathrm{p} \pi^{*}$ peak shifting 2.6 $\mathrm{eV}$ to higher energy. This indicates that the positive charge of the $\mathrm{CO}^{+}$ion is mainly at the carbon site.

\subsubsection{Molecules with three atoms}

In this section we discuss some molecular systems with three atoms. The general interpretation of the oxygen $\mathrm{K}$ edge remains similar to the binary molecules, that is one observes the lowest empty orbitals and at higher excitation energies they are mixed with Rydberg states. Figure II shows the molecular orbitals of $\mathrm{O}_{3}, \mathrm{CO}_{2}$ and $\mathrm{NO}_{2}$.

$\mathrm{O}_{3}$ : Ozone, $\mathrm{O}_{3}$, is a bent molecule with a central oxygen atom and two equivalent end oxygens. This creates a new situation compared to $\mathrm{O}_{2}$ because it is now possible to excite a is core electron from two different oxygen 

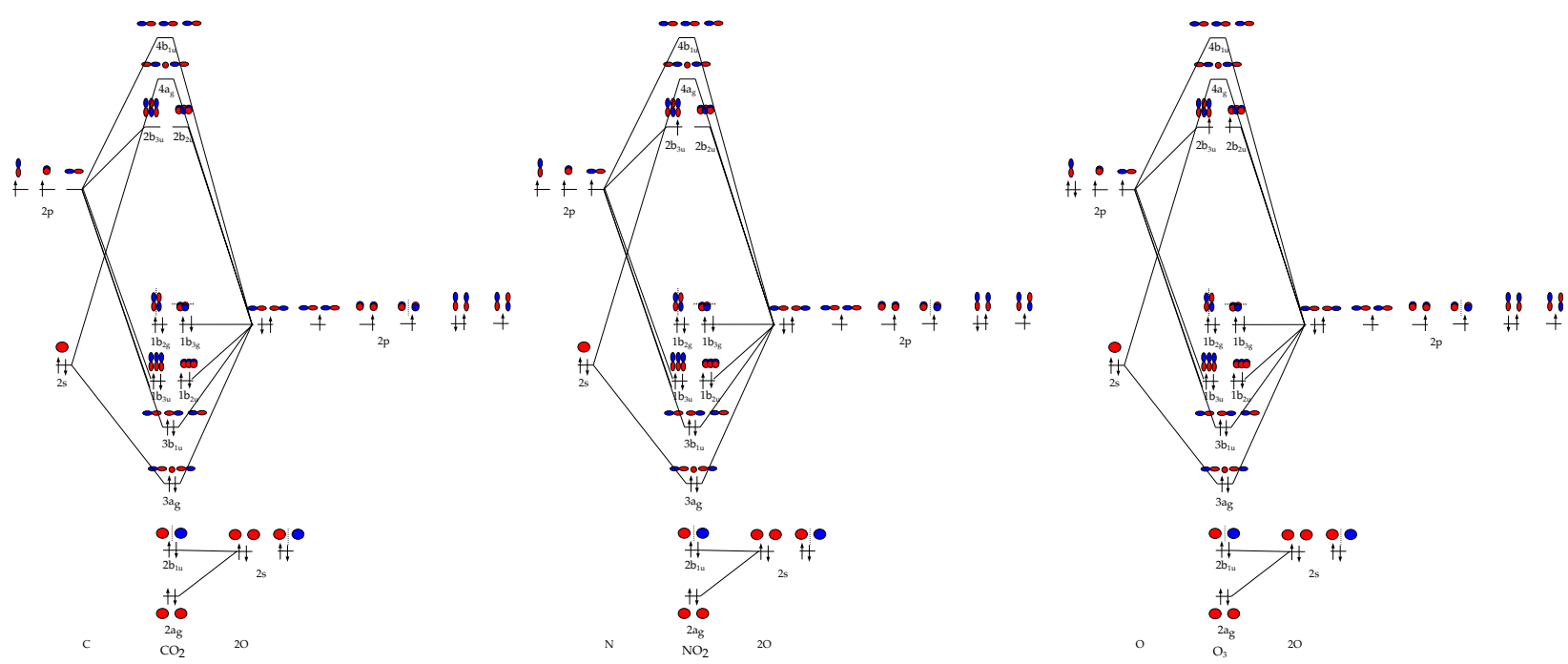

Figure 11: Molecular orbital diagrams of $\mathrm{CO}_{2}, \mathrm{NO}_{2}$ and $\mathrm{O}_{3}$. The $\mathrm{MO}$ schemes are equivalent with the difference that the number of electrons in the $2 \mathrm{p} \pi^{*}$ orbital is respectively 0,1 and 2 .

atoms. The molecular orbitals of $\mathrm{O}_{3}$ can be divided into $\pi$ orbitals and sigma orbitals. The $\pi$ orbitals for bonding, non-bonding and antibonding combinations, with only the $2 \mathrm{p} \pi^{*}$ anti-bonding orbital empty. At higher energy there are two $2 p \sigma^{*}$ anti-bonding orbitals. The $\mathrm{O}_{3}$ oxygen $\mathrm{K}$ edge spectrum shown in Figure 12 shows two sharp peaks appear at 529 and $536 \mathrm{eV}$ [172]. The first sharp peak has been ascribed to the transition from the is electron of the terminal oxygen to the $2 \mathrm{p} \pi^{*}$.

The second sharp peak at $536 \mathrm{eV}$ is ascribed to transitions from the $1 \mathrm{~s}$ electron of the central oxygen to the $2 \mathrm{p} \pi^{*}$. Note that both the peaks at respectively 529 and $536 \mathrm{eV}$ originate from the same molecular orbital, still they appear at $7 \mathrm{eV}$ difference in their excitation energy because the terminal oxygen atoms is much lower in energy. In other words the central oxygen atom is more positively charged. The complete analysis shows that the second peak also has some intensity from transitions into the $2 \mathrm{p} \sigma^{*}$ anti-bonding orbitals for the terminal oxygens. The peaks at 540 to $545 \mathrm{eV}$ are due to transitions into the (mixed) $2 \mathrm{p \sigma}^{*}$ anti-bonding orbitals and to Rydberg transitions. To summarise, it is interesting to compare the assignments for the oxygen atom and the $\mathrm{O}_{2}$ and $\mathrm{O}_{3}$ molecules.

- The oxygen atom only has the main $2 \mathrm{p}$ peak and at higher energy the Rydberg states.

- The $\mathrm{O}_{2}$ molecule has different chemical states, respectively the $2 \mathrm{p} \pi^{*}$ and $2 p \sigma^{*}$ peaks. 
- The $\mathrm{O}_{3}$ molecule has different oxygen atoms, respectively at the center and at the edge of the $\mathrm{O}_{3}$ molecule, yielding different peaks related to the same $2 \mathrm{p} \pi^{*}$ orbital due to different core hole potentials.
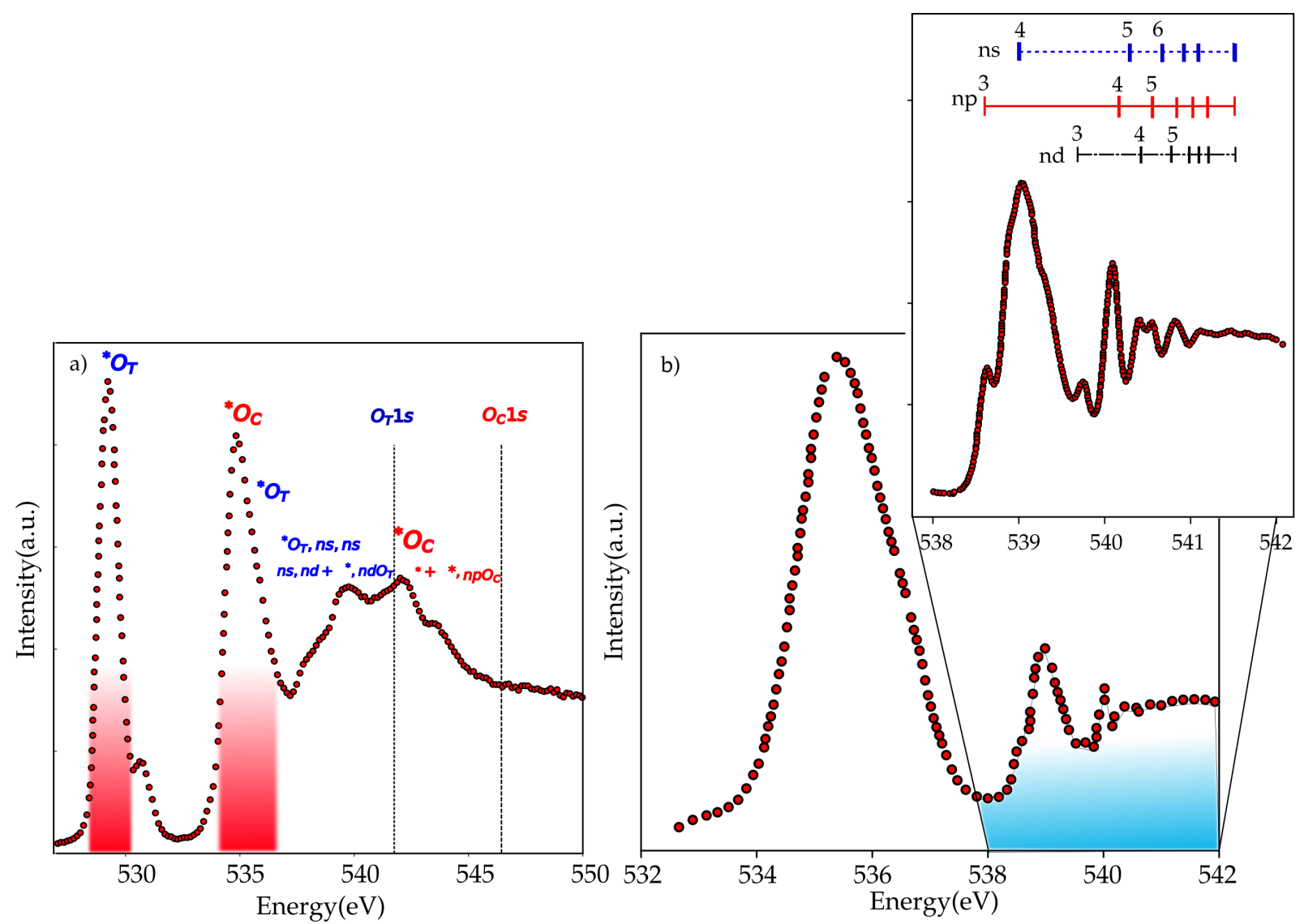

Figure 12: a) Oxygen K edge TIY spectrum of ozone [172]. The peaks at $529 \mathrm{eV}$ and $536 \mathrm{eV}$ are excitations into the same $2 \mathrm{p} \pi^{*}$ state, from respectively the terminal $\mathrm{O}_{\mathrm{T}}$ and the centre oxygens $\mathrm{O}_{\mathrm{C}}$. Note that the small peak at 531 $\mathrm{eV}$ is due to the presence of some $\mathrm{O}_{2} \cdot \mathrm{b}$ ) Oxygen $\mathrm{K}$ edge spectrum of $\mathrm{CO}_{2}$ from ref. [173]. The $2 \mathrm{p} \pi^{*}$ state is seen at $535 \mathrm{eV}$. Above $538 \mathrm{eV}$ the $2 \mathrm{p} \sigma^{*}$ and the Rydberg states are visible.

$\mathrm{NO}_{2}$ : Nitrogen dioxide is a bent molecule with a central nitrogen atom and two equivalent end oxygens, in other words it has a similar structure to $\mathrm{O}_{3}$. This implies that also the molecular orbitals of $\mathrm{NO}_{2}$ are similar to those of $\mathrm{O}_{3}$ and the $\pi$ orbitals form bonding, non-bonding and antibonding combinations. Because there is one electron less the non-bonding $\pi$ orbital contains only one electron. The oxygen $\mathrm{K}$ edge of $\mathrm{NO}_{2}$ contains two sharp peaks, respectively related to the non-bonding and anti-bonding $\pi$ orbitals. At higher energy we observe broad structures due to the $\sigma$ orbitals and Rydberg states. It is interesting to note that both $\mathrm{NO}_{2}$ and $\mathrm{O}_{3}$ have two $\pi$ 
related peaks, but for $\mathrm{NO}_{2}$ they relate to two different orbitals, while for $\mathrm{O}_{3}$ they relate to the same orbital, but a is core hole on two different oxygen atoms, center respectively end.

Renner Teller effect: The break down of the adiabatic approximation in triatomic molecules is called the Renner Teller effect.

The experimental manifestation of this effect is an irregular vibrational structure of the spectra that reflects the coupling of two electronic states in the bent nuclear conformations which were degenerate at the linear molecular geometry. The Renner Teller effect is a consequence of the electrostatic interaction between two components of an electronic state with a non-zero angular momentum.

$\mathrm{CO}_{2}$ : Carbon dioxide is a linear molecule with the bonding and non-bonding $2 p$ orbitals occupied. It is a closed shell system that has a ground state term symbol ${ }^{1} \Sigma_{\mathrm{g}}^{+}$. The lowest three empty states are respectively the double degenerate $2 \mathrm{p} \pi^{*}$ state, followed by two anti-bonding $2 p \sigma^{*}$ states. The $2 \mathrm{p} \pi^{*}$ state is doubly degenerated but upon the oxygen $\mathrm{K}$ shell excitation the equilibrium bond angle is changed and the degeneracy is removed by the Renner-Teller effect $[162,174]$. Because of the energy lowering resulting from bending the structure, the $2 \pi_{\mathfrak{u}}$ splits in two components. The first peak in Figure 12 corresponds to the promotion of an oxygen core electron to the two component of the lowest empty molecular orbital $2 \pi_{\mathfrak{u}}$. This peak presents a large FWHM compared to the elastic peak [162], which suggests that not only the excitation from the $K$ shell to the $2 \pi$ orbital contributes to this peak but also the lowest $1 s \sigma_{\mathfrak{u}} \longrightarrow 3 s \sigma_{g}$ Rydberg transition with antibonding valence character [173].

This hypothesis is supported by the fact that this transition is optically allowed. The peaks in the higher energy part of the spectrum can be assigned to Rydberg transitions [173]. Thus all the $\mathrm{p} \pi, \mathrm{p} \sigma$ and $\sigma$ transitions are allowed. This can lead to the conclusion that a mixing of the valence state with the $\sigma^{*}$ antibonding resonance occurs [174].

Shieh et al. studied the oxygen $\mathrm{K}$ edge of $\mathrm{CO}_{2}$ under pressure [175]. The $\mathrm{p} \pi$ peak disappears at $37 \mathrm{GPa}$ and it is replaced by two structures at respectively $532 \mathrm{eV}$ and $540 \mathrm{eV}$. At pressures above $37 \mathrm{GPa} \mathrm{CO}_{2}$ does not exist as separate molecules, but has polymerised.

There are several options for the condensed phase of $\mathrm{CO}_{2}$, with the phase 
where carbon is fourfold coordinated can best explain the observed spectral changes [175].

\subsubsection{Water}

$\mathrm{H}_{2} \mathrm{O}$ is studied in the gas, liquid and solid phase. In this section we briefly discuss all the states of water.

The oxygen $\mathrm{K}$ edge of water is a very active research field and we do not intend to cover all developments but focus only on the basic components in the analysis. Readers are referred to recent reviews on the x-ray spectroscopy of water, in particular the recent reviews by Smith and Saykally [176] and by Nilsson and Petterson [177]. Gas phase $\mathrm{H}_{2} \mathrm{O}$ is a bent molecule with five occupied orbitals. The highest occupied molecular orbital, is a non-bonding $1 b_{1}$ orbital derived from the oxygen $2 p_{x}$ orbital without contributions from the hydrogen is orbital, in other words it is the "lone pair". At lower energy the orbitals are respectively from highest to lowest energy the $3 \mathrm{a}_{1}, 1 \mathrm{~b}_{2}$ and $2 \mathrm{a}_{1}$ molecular orbitals with contributions from hydrogen is and oxygen $2 \mathrm{~s}$ and $2 \mathrm{p}$ orbitals, i.e. participating in the $\mathrm{O}-\mathrm{H}$ bond. The two lowest unoccupied molecular orbitals are the $\mathrm{O}-\mathrm{H} 4 \mathrm{a}_{1}$ and $2 \mathrm{~b}_{2}$ antibonding orbitals.

Water molecules in liquid and solid phases show two different types of $\mathrm{O}-\mathrm{H}$ interactions: (1) covalent intramolecular $\mathrm{O}-\mathrm{H}$ bonding and (2) weak intermolecular hydrogen bonds [178].

The study of the oxygen $\mathrm{K}$ edge allows the investigation of the unoccupied states, most importantly the antibonding $\mathrm{O}-\mathrm{H}$ molecular orbitals and their character that is sensible to the $\mathrm{H}$-bonding network around the probed oxygen atom. The oxygen $\mathrm{K}$ edge spectrum of water is considered as being formed by three regions: the pre edge region at $535 \mathrm{eV}$, the main edge region around $537 \mathrm{eV}$ and the post edge region at $541 \mathrm{eV}$. These regions go through modifications depending on the water phase. In figure 13 the oxygen $\mathrm{K}$ edge spectra of ice, liquid and gas phase water are compared. The gas phase spectrum shows the anti-bonding orbitals in the molecular MO scheme. In case of the liquid state, the pre-edge has been ascribed to the presence of broken H-bonds by computational and experimental studies [180], the main edge region has been ascribed to interstitial water molecules [56]. The postedge region contains features that consist of states delocalized trough the H-network and results from a tetrahedral ice-like coordination [87].

Cavalleri et al. investigated the spectral change for the pre and main edge going from the free molecule to the pentamer. They highlighted the role of 


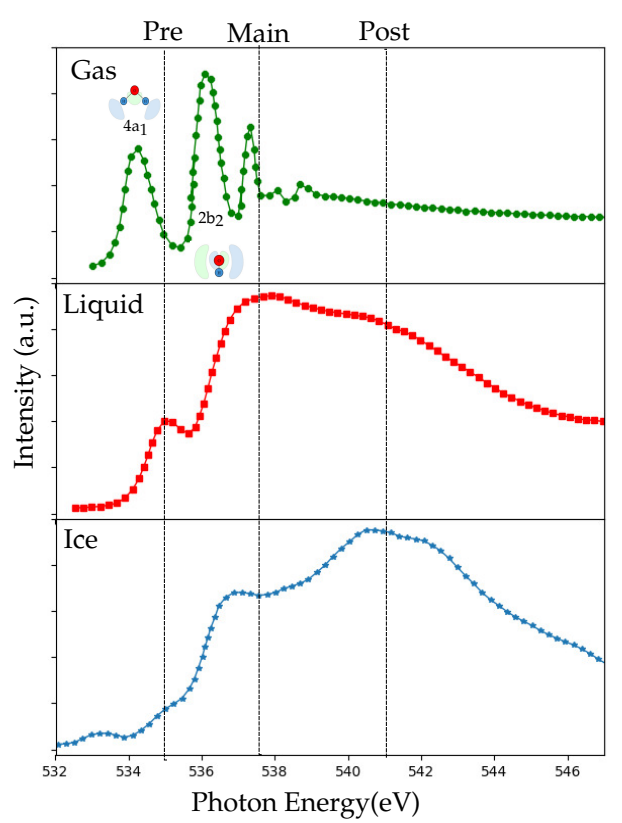

Figure 13: X-ray absorption spectra of gas phase water, liquid water at $299 \mathrm{~K}$ and ice grown on $\mathrm{BaF}_{2}$ at $144 \mathrm{~K}$ [179].

the H-bond directionality on the spectral shape: the unoccupied antibonding $\mathrm{O}-\mathrm{H}$ orbitals, in gas phase, are polarized towards the hydrogen, so they have a strong sensitivity to additional H-bonds [181]. The features that constitute the post-edge region have been related to states that are localized along the H-bond. These states give rise to the conduction band in the ice phase with a strong intensity due to the fact that both $\mathrm{OH}$ groups are involved in donor hydrogen bonds [182]. The three different regions of the water spectra are affected by several factors such as a change in temperature, different solvents and isotope effects. Heating the liquid will cause the post edge region to lose intensity while the pre-edge and the main-edge will increase their intensity. The study of Wernet et al. [183] gave rise to a huge debate on the local structure of liquid water at different temperatures.

It has been concluded that the spectrum of liquid water is composed of two different species: a species with two hydrogen bonded configurations with one strong donor and one strong acceptor bond donating H-bond and a second species that is more symmetric with a tetrahedral-like coordination close to the ice coordination.

Increasing the temperature, the water structure diverges from the tetrahedral like coordination. Also different solvents such as benzene, acetonitrile and chloroform [184] can be seen as hydrogen bonding network perturba- 
tions that allow symmetrical bonding and promote ice-like structures with a consequent increase in the post edge structure.

Fuchs at al. $[185,186]$ observed a significant isotope influence in the spectra of liquid water: the pre edge peak shows a blue shift in the $\mathrm{D}_{2} \mathrm{O}$ spectra due to the different zero point energies of the ground states and a reduced intensity in the pre edge region of the deuterated species due to smaller distortion of the bonding network.

\subsubsection{Bio-organic molecules}

The general interpretation of the oxygen $\mathrm{K}$ edges of large molecules is similar to the small molecule discussions above. The peaks in the oxygen $\mathrm{K}$ edge are analysed in terms of the lowest empty orbitals, where in case of two or more non-equivalent oxygen atoms in a molecule, the individual spectra of the oxygen atoms are added, taking into account that there can be different excitation energies into the same orbital. A large number of oxygen $\mathrm{K}$ edge spectra are given in the COREX database [187].

XAS studies of bio-organic molecules are difficult due to the risk of beam
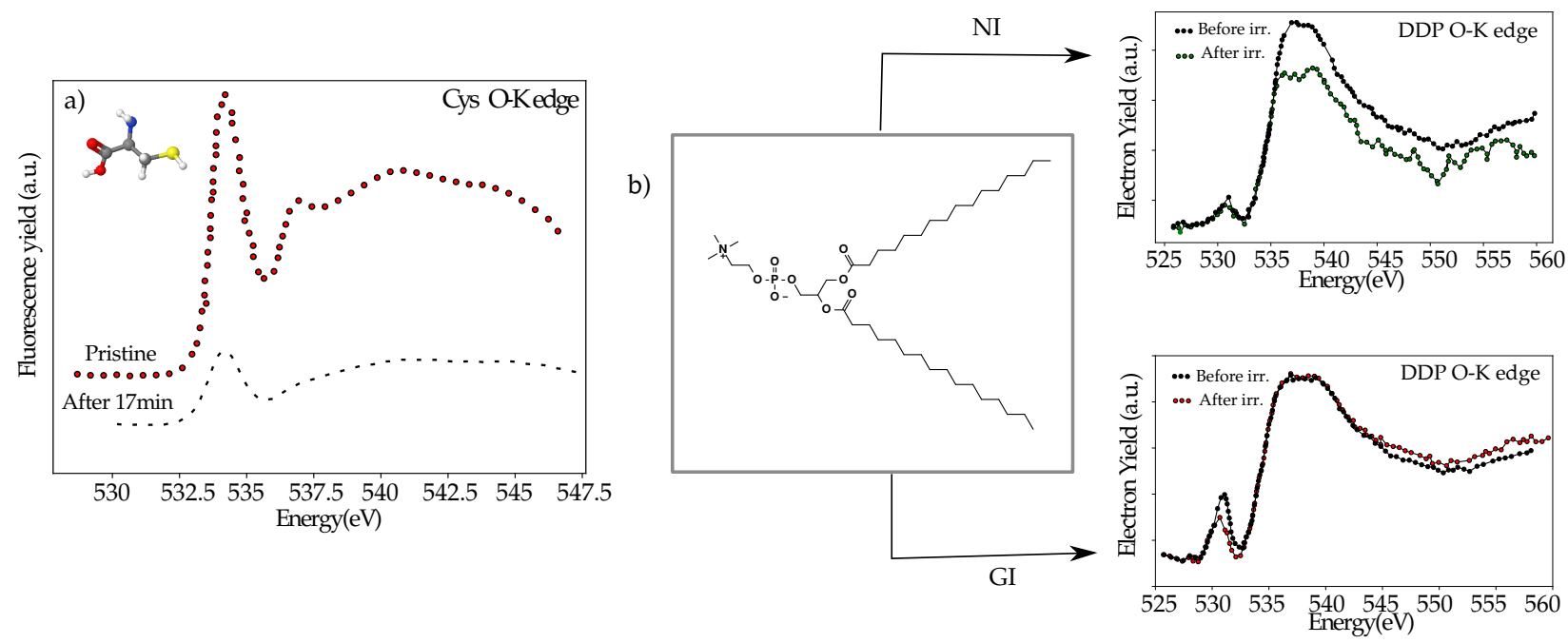

Figure 14: (a) Time evolution of the oxygen K edge spectra of cysteine at time zero and after 17 minutes without any rescaling [188]. (b) Oxygen K edge of DPPC at grazing incidence (GI) and normal incidence(NI) before and after the irradiation [189].

damage [188]. The main degradation processes that can occur when measuring the oxygen $\mathrm{K}$ edge can be grouped as [188]: (a) dehydrogenation due to the $\mathrm{O}-\mathrm{H}$ group, (b) dehydration, i.e. the loss of water molecules because of 
the $\mathrm{C}-\mathrm{OH}$ bond, (c) decarboxylation (loss of molecular $\mathrm{CO}_{2}$ ) and (d) decarbonylation, the loss of molecular $\mathrm{CO}$.

Zubachius et al. [188] investigated the radiation induced modification in the oxygen $\mathrm{K}$ edge spectra of cysteine (Figure 14 ). The spectral shape is less affected by the beam damage than the intensity, the spectra recorded after 17 min of exposure has an intensity of one order of magnitude lower than the pristine system. This implies that at least $90 \%$ of the oxygen present in the molecule has been detached as $\mathrm{H}_{2} \mathrm{O}$ or $\mathrm{CO}_{2}$. Radiation damage can manifest itself in the oxygen $\mathrm{K}$ edge measurements also as perturbation of the supramolecular structure [190, 191].

Panajotović et al. investigated the radiation damage on phospholipidic membranes and they tested the impact of different incidence modes on the spectra: many broken $\sigma$ bonds especially in normal mode and new features appeared [189].

Other common chemical modifications induced by ionizing radiation are the formation of free radicals [192, 193], where the reduction product is usually formed by the addition of an electron to the carbonyl oxygen of the carboxyl group [192, 194-196] and mass loss phenomena, [197, 198] depending on factors such as the initial mass, the dose rate and the area of irradiation [197].

Amino acids, polypeptides and proteins: The measurement of the oxygen $\mathrm{K}$ edge $\mathrm{XAS}$ in amino acids is a powerful tool since oxygen can be in different bonding environments and different chemical shifts for each oxygen in amino acids are observed [200]. In Figure 15 the oxygen K edge spectra of all the 22 amino acids are reported [199]. The spectra show a dominant peak that has been assigned to the $\pi^{*}$ resonance followed by the $\sigma^{*}$ resonance at higher energy. In the aspartic acid spectra, a peak around 534.0$534.5 \mathrm{eV}$, with an energy difference from the main peak of $1.5 \mathrm{eV}$ has been reported and assigned to the is $\rightarrow \pi^{*}$ excitation of the $\mathrm{OH}$ oxygen in carboxy group [199-201]. Also the glutamic acid shows a similar feature [199]. From the hydroxyl group of serine, threonine and hydroxyproline, a $\sigma^{*}(\mathrm{O}-\mathrm{C})$ transition occurs in the range $\approx 538-540 \mathrm{eV}$ [199]. A probable contribution from residual water is expected in the energy range 537-540 eV [199, 202]. Even if isolated amino acids in gas phase are always neutral, in solution any change in $\mathrm{pH}$ can bear dramatic changes in their charge state as they can exist as cations at low $\mathrm{pH}$ values, charge neutral zwitterions at intermediate $\mathrm{pH}$ values, and anions in basic solutions.

The addition or removal of a proton affects the local symmetry of the electric 


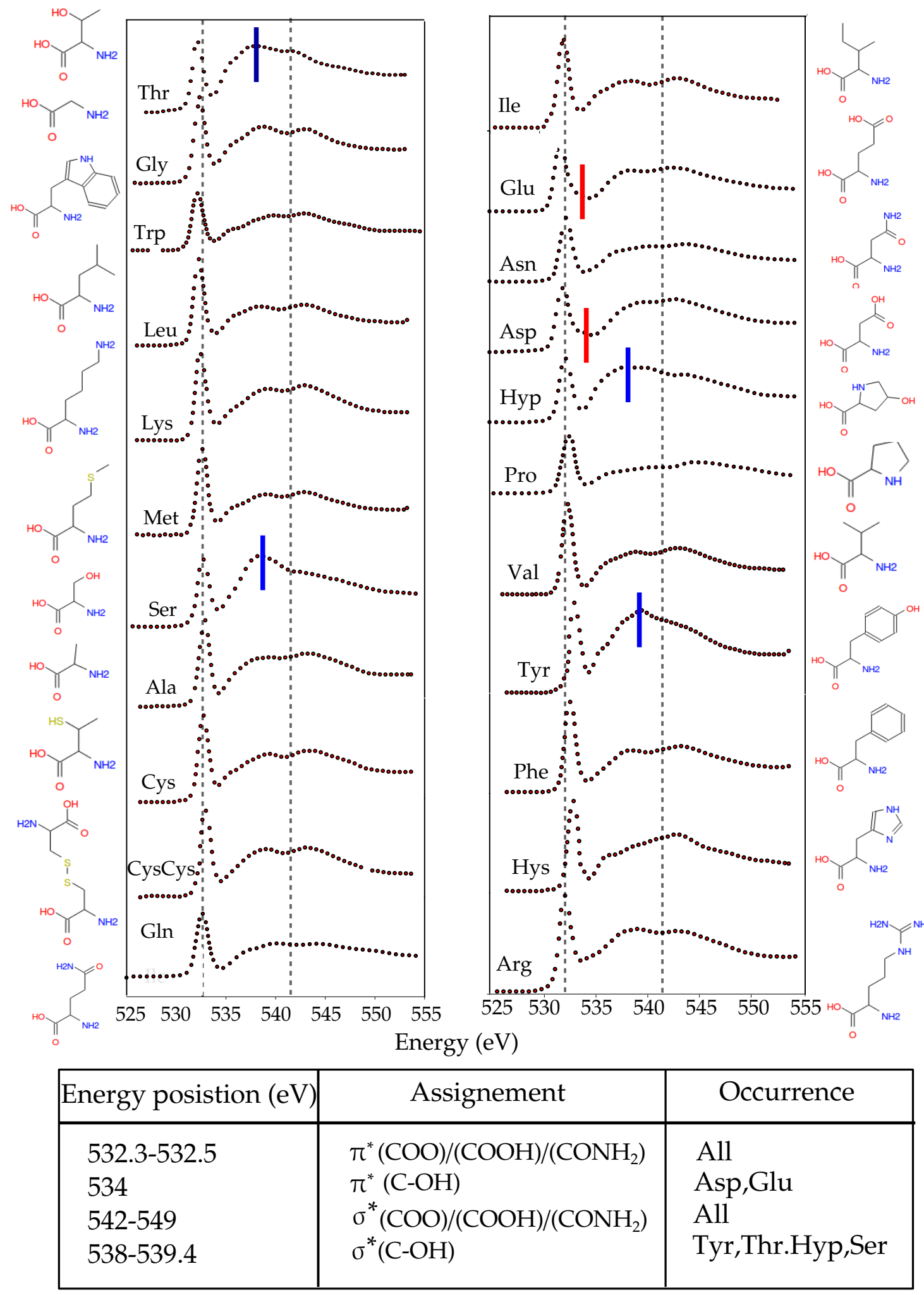

Figure 15: Experimental oxygen K-edge spectra of the 22 amino acids and their molecular formula [199]. In the table the peak position together with their assignment are reported. 
field surrounding the terminal oxygen [202], so the energy levels of molecular orbitals at the carboxylic group would be modified by any $\mathrm{pH}$ change.

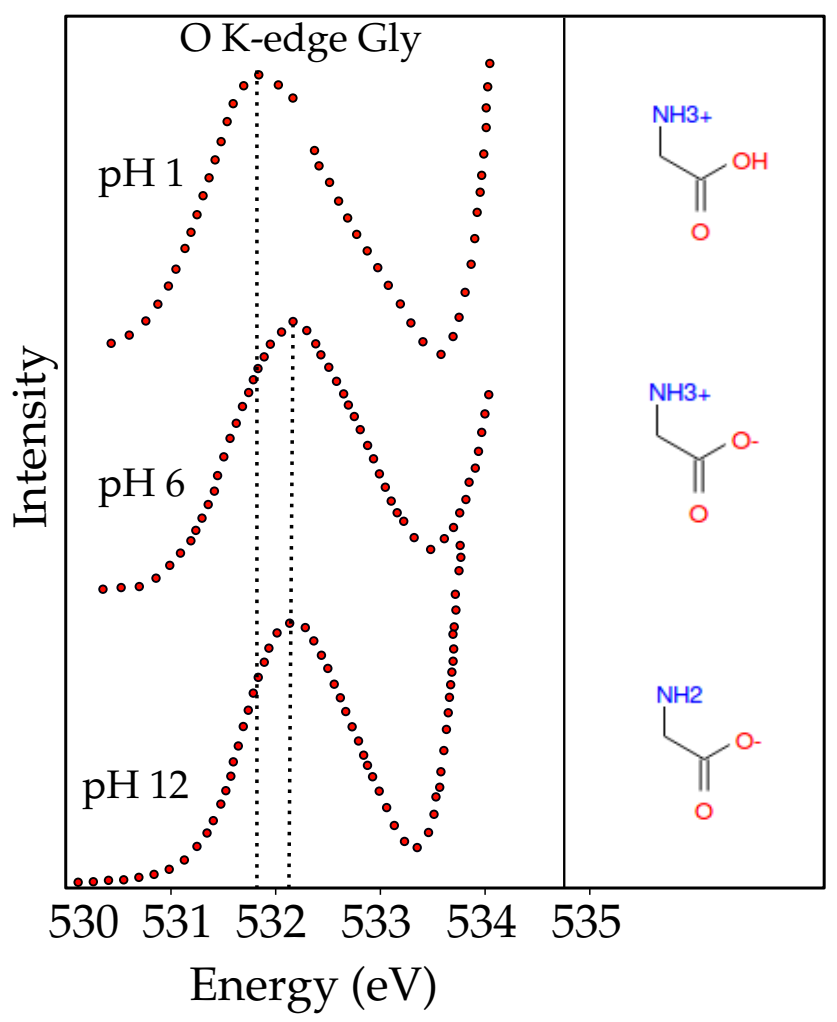

Figure 16: Oxygen K edge spectra of $0.6 \mathrm{M}$ glycine (aq) as a function of $\mathrm{pH}$. A small red shift is observed for the acidic solution, which has been illustrated by a dotted line through the center of each peak. This shift is caused by the protonation of the carboxylate subgroup at low $\mathrm{pH}$, and the resultant breaking of the degeneracy in the O1s $\rightarrow \pi_{\mathrm{CO}}^{*}$ transition[202].

In Figure 16 the spectra in solution of glycine at different $\mathrm{pH}$ values is reported. The terminal carboxyl group of glycine is protonated in an acid environment so a lift of the degeneracy $\mathrm{CO}$ double bond and a feature around $535 \mathrm{eV}$ due to the $\mathrm{C}-\mathrm{O}$ bond is expected. The strong background from the aqueous solution cannot be easily subtracted and makes the attempt to identify further resonances difficult[202].

DNA components: In Figure 17 the oxygen K edge XAS spectra of uracil $(\mathrm{U})$, thymine $(\mathrm{T})$, cytosine $(\mathrm{C})$ and guanine $(\mathrm{G})$ are reported[203]. The bases show a strong feature around $532 \mathrm{eV}$ arising from the carbonyl group $\left(\pi_{\mathrm{CO}}^{*}\right)$, and a $\sigma *$ resonance above $535 \mathrm{eV}$, thymine shows a splitting of the $\pi^{*}$ main resonance due to an energy differnce of teh two $\mathrm{C}=\mathrm{O}$ bonds [204-206]. Also the uracil spectra has a splitting in the first $\pi^{*}$ peak, but less pronounced; the stronger blue shift of the $\pi *_{2}$ of thymine is related to the substitution effect of the methyl group. DNA is built up by nucleobases 
attached to ribose sugar and phosphate groups. The way the sugar and the phosphate group effects the spectra of oxygen K edge of thymine is shown in Figure 17. With the addition of sugar and phosphate the spectrum acquires features characteristic of the phosphate group as the enhancement of the $\sigma *(\mathrm{C}-\mathrm{O})$ structure $[203,204]$. The small peak in the $\pi^{*}$ region comes only from the base [203].
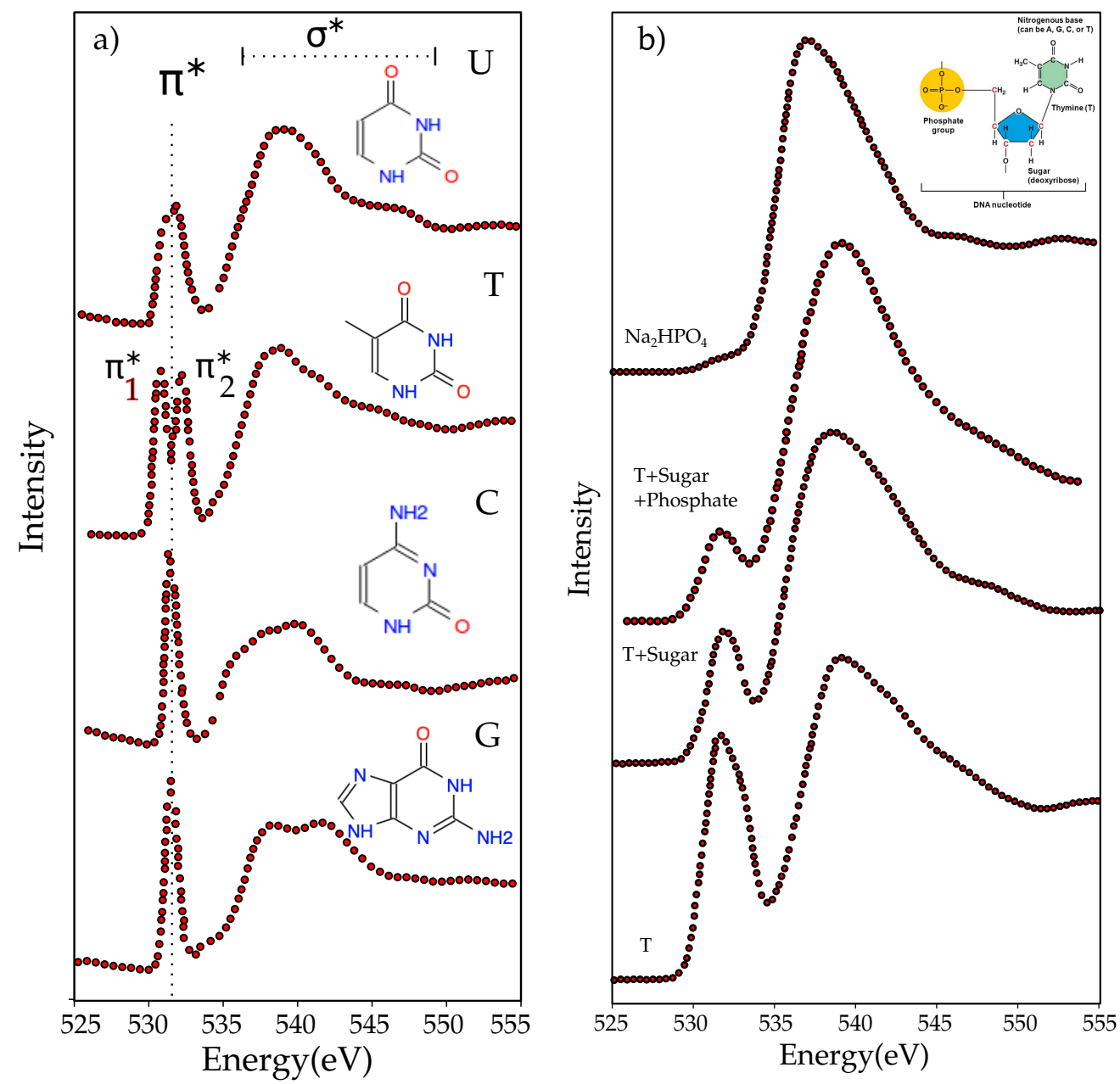

Figure 17: Figure a) Oxygen $\mathrm{K}$ edge spectra of uracile, thymine, citosine and guanine together with their molecular structure and the peak assignment[203]. Figure b) Effect on the oxygen K edge spectra from adding sugar and phosphate groups of DNA to thymine[204]

\subsection{ADSORPTION}

\subsubsection{Atomic adsorption}

Molecules adsorbed on a surface change their electronic structure and the oxygen $\mathrm{K}$ edge is a useful tool to study the adsorption processes of oxygen- 
containing molecules. The adsorption phenomena of small molecules are particularly important as the fundamental steps in catalytic processes.

The interaction of oxygen atoms with a surface is strong due to the bonding of the oxygen $2 p$ orbitals with the metal orbitals near the Fermi level. Three $2 p$ orbitals are involved in the surface chemical bond with an adsorbing atom.

The interaction with the $2 \mathrm{p}_{z}$ orbital has $\sigma$ symmetry with respect to the surface. The $2 p_{x}$ and $2 p_{y}$ orbitals are parallel to the surface and can only interact with the surface $\mathrm{s}$ atomic orbitals in high coordination sites. This bonding interaction contributes significantly to the total surface bond energy implying that bonding to high coordination sites is favoured. The $2 p$ orbitals form bonding and anti-bonding orbitals with the metal orbitals.

Figure 18 shows the oxygen $\mathrm{K}$ edge of oxygen adsorbed on a $\mathrm{Cu}(100)$ sur-

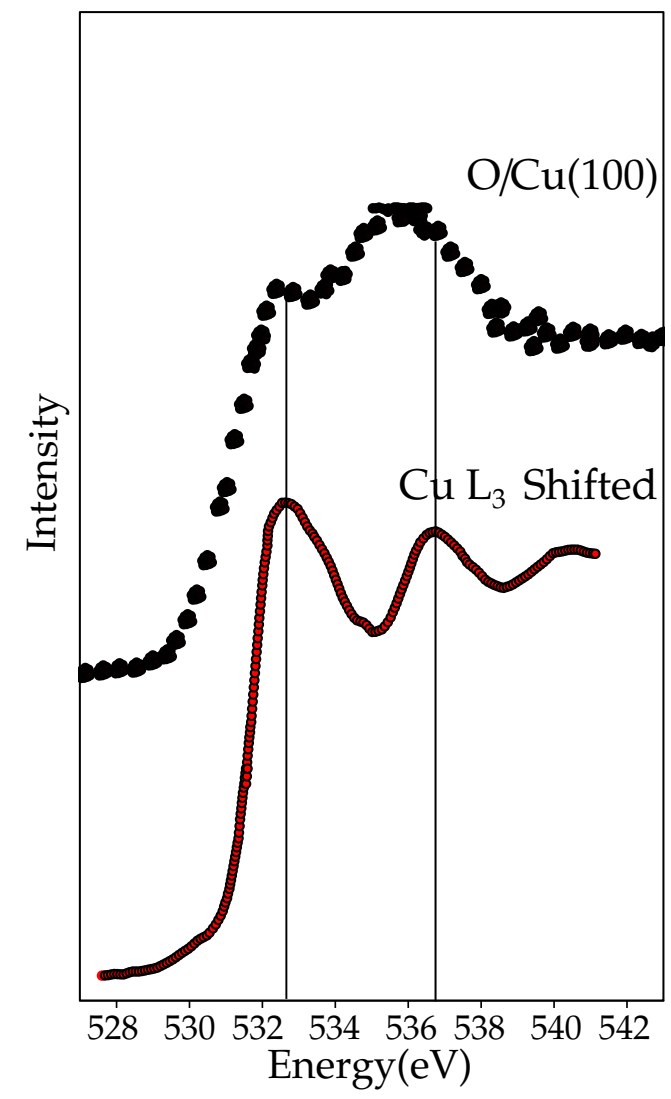

Figure 18: The oxygen $\mathrm{K}$ edge of oxygen adsorbed on a $\mathrm{Cu}(100)$ surface (points) [207] compared with the $\mathrm{Cu} \mathrm{L}_{3}$ edge shifted by $401 \mathrm{eV}$ [208]

face (points) compared with the energy shifted copper $2 p$ XAS spectrum of copper metal.

The atomic energy of the $2 p$ orbital is below the Fermi level, implying that the anti-bonding combinations will have mainly metal character. The oxygen $\mathrm{K}$ edge in first approximation maps the empty metal states, slightly shifted 
to higher energy due to the anti-bonding character. From this analysis, one would expect that if an oxygen atom is adsorbed on a copper surface, in first approximation the oxygen $\mathrm{K}$ edge will map the empty metal states and as such it will look similar to the copper $2 p$ XAS spectrum that maps the empty copper s states.

\subsubsection{Molecular adsorption}

X-ray absorption of the adsorption of molecules to surfaces has been discussed in detail in the book Chemical bonding at surfaces and interfaces by Nilsson, Pettersson and Norskov [209]. The adsoption of oxygen-containing molecules to a surface is weaker than that of oxygen atoms.

In a molecule the atom that binds to the surface is still bonded to other atoms from the molecule. The adsorbed atom divides its bonding strength between the molecule and the surface, with the consequence of weaker bonding than an isolated oxygen atom. One can distinguish physisorption and chemisorption. In chemisorption there is a chemical bond between the molecule and the surface, while in physisorption the molecule is attached to the surface without a chemical bond, for example via van der Waals interactions.

CO on metal surfaces: The bonding of $\mathrm{CO}$ on $\mathrm{Ni}(100)$ was studied in detail in 1982 by Stohr and Jaeger [210]. One observes the transitions to the $2 \pi^{*}$ orbital at $534.0 \mathrm{eV}$ and to the $\sigma^{*}$ orbital at $550.0 \mathrm{eV}$. In the free $\mathrm{CO}$ molecule, these excitation energies are respectively $534.2 \mathrm{eV}$ and $550.9 \mathrm{eV}$ (see Table 3), implying that the $2 \pi^{*}$ excitation energy does not shift while the $\sigma^{*}$ orbital shifts by $0.9 \mathrm{eV}$. Thus, the energy difference between the $2 \pi^{*}$ and the $\sigma^{*}$ orbitals is reduced from $16.8 \mathrm{eV}$ in free $\mathrm{CO}$ to $16.0 \mathrm{eV}$ in $\mathrm{CO} / \mathrm{Ni}(100)$. This energy reduction can be ascribed to the shift to higher energy by the antibonding combination of the $2 \pi^{*}$ orbital and the Ni $3 \mathrm{~d}$ orbitals.

Figure 19 shows an angular dependence. At normal incidence the $2 \pi^{*}$ peak is strong because the electric field is aligned with the $2 \pi^{*}$ resonance. At grazing incidence the electric field is parallel to the $\mathrm{CO}$ axis, hence perpendicular to the $2 \pi^{*}$ orbital and the $2 \pi^{*}$ resonance is small. The argument is reversed for the $\sigma^{*}$ orbital that has the highest intensity when the electric field is parallel to the $\mathrm{CO}$ axis. This angular dependent effect, also known as linear dichroism, indicates the bonding orientation of the adsorbed molecule.

Tillborg et al. studied the oxygen K edge XAS spectra of $\mathrm{CO}$ adsorbed on three different sites on a $\mathrm{Ni}(100)$ surface, respectively hollow sites, bridge 
sites and on-top sites. The energy position and relative intensity of the oxygen $\mathrm{K}$ edge indicates the difference in chemical bonding [211].

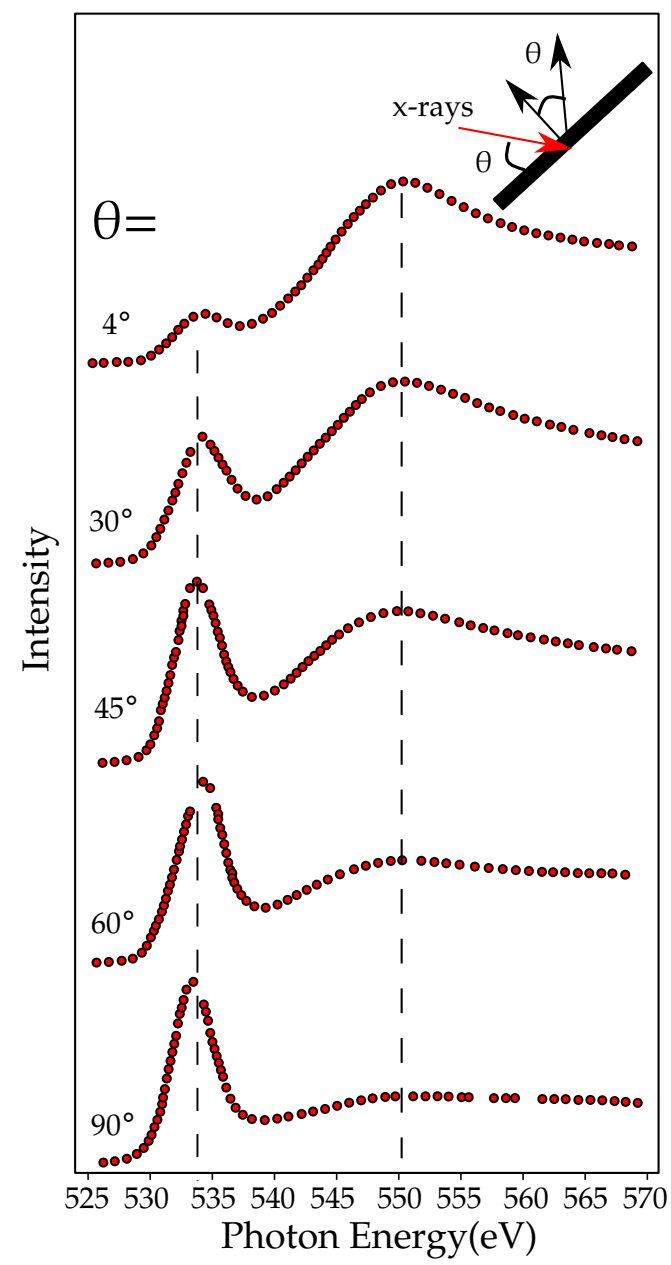

Figure 19: The oxygen $\mathrm{K}$ edge of $\mathrm{CO}$ on $\mathrm{Ni}(100)$ [210]. The $2 \pi^{*}$ orbital is visible at $534.0 \mathrm{eV}$ and to the $\sigma^{*}$ orbital at $550.0 \mathrm{eV}$.

\section{Linear Dichroism}

Linear Dichroism or Polarization Dependent x-ray absorption concerns the difference in XAS spectral shape of a system as a function of its angle with respect to the x-rays.

At a bending magnet beamline, the $\mathrm{x}$-ray is linearly polarized in the plane of the synchrotron. This implies that the $x$-ray excites the electric dipoles that are aligned in this plane. Assuming a planar molecule lying on a flat surface, one can excite the planar bonds if the $\mathrm{x}$-ray polarization vector lies in this plane.

The bonds perpendicular to the plane are excited when the sample is turned with the electric vector perpendicular to the molecular plane. This yields two polarization dependent 
spectra and the difference spectrum is the Linear Dichroism spectrum. If the symmetry of the system is broken by a magnetic field, there will also be linear dichroism, which then is called Magnetic Linear Dichroism (MLD).

\subsection{ANALYSIS OF CATALYTIC REACTIONS}

The information on the adsorbed species can be used in the analysis of oxygen $\mathrm{K}$ edges of catalytic reactions.

One can study the oxygen K edge XAS spectra under in-situ conditions. The most common approach uses conversion electron yield, i.e. the excited electrons ionise the gas phase and the resulting ionised ions are detected. Often these experiments are performed in so-called near-ambient-pressure XPS set-ups, implying that the gas pressure is in the mbar range [82], but one could also use 1 bar pressures in dedicated in-situ XAS reactors.

Knop-gericke et al. studied methanol oxidation to formaldehyde over copper metal [82]. One detector tracks the oxygen components in the gas phase and a second detector tracks the oxygen components of the surface and gas phase combined, allowing the determination of the surface species by subtracting the pure gas phase spectrum. The oxygen $\mathrm{K}$ edge as a function of temperature indicates a transition from $\mathrm{Cu}_{2} \mathrm{O}$ at $540 \mathrm{~K}$ to a species that is described as adsorbed oxygen atoms that mainly interact with the copper $\mathrm{s}$ states. The different oxygen species are then correlated with the catalytic reactions [82]. Pfeifer et al. performed a detailed study on the comparison between amorphous and crystalline rutile $\mathrm{IrO}_{2}$, which is important regarding its behaviour as electrocatalyst for the oxygen evolution reaction [212]. The rutile phase is a pure $\mathrm{Ir}^{4+} \mathrm{O}^{2-}$ oxide and the oxygen $\mathrm{K}$ edge shows two peaks: a sharp peak at $530 \mathrm{eV}$ related to the empty Ir $5 \mathrm{~d}$ states and a broad peak at $533 \mathrm{eV}$ due to the sp-band. The amorphous $\mathrm{IrO}_{x}$ shows additional fine structure in the $5 \mathrm{~d}$ spectral region, which in the paper is assigned to partial reduction to $\mathrm{Ir}^{3+}$, due to the formation of Ir vacancies. In our opinion, a shift to lower energy in the oxygen $\mathrm{K}$ edge should be related to an oxidation to $\mathrm{Ir}^{5+}$, in other words the $\mathrm{Ir}$ vacancy creates $\mathrm{Ir}^{5+}$ sites at neighbouring positions, which causes a shift to lower binding energy.

Tesch et al. studied the behaviour of $\mathrm{MnO}_{x}$ electrocatalysts as a function of the potential [213]. The oxygen $\mathrm{K}$ edge of the $\mathrm{MnO}_{x}$ system changes as a function of the potential, which is analysed as a change in the Mn-O hybridization. In our opinion what is visible in the oxygen $\mathrm{K}$ edge is mainly the change of the ratio of $\mathrm{MnO}_{x}$ and nafion/graphene background and the 
oxygen $\mathrm{K}$ edge related to the $\mathrm{MnO}_{x}$ species remains the same for all potentials. 
COUPLED CLUSTER STUDY OF THE X-RAY

ABSORPTION SPECTRA OF FORMALDEHYDE

DERIVATIVES

Calculations of core excitations are challenging because they require computational methods that explicitly account for the excitation of core-level electrons, including relaxation and electron correlation effects (see Chapter 1 ).

In the realm of electronic structure wave-function based methods, the methods rooted in a coupled cluster ansatz for the wave function [214-217] where the correct description of the quantum mechanical system can be approached in a systematic manner, are generally considered among the most accurate. Implemented within response theory, coupled cluster (CC) methods give a reliable theoretical framework in which relaxation effects are taken into account by means of an accurate treatment of electron correlation in both the ground and excited states.

In this chapter the performance of the coupled cluster hierarchy of methods CCS (coupled cluster singles), CC2 (coupled cluster singles and approximate doubles) and CCSD (coupled cluster singles and doubles) with a corevalence separation (CVS) scheme applied within the manifold of excited states (in the following labelled eCVS) [218] is evaluated on the carbon, oxygen and fluorine $\mathrm{K}$ edge XAS spectra of the molecules formaldehyde $\left(\mathrm{CH}_{2} \mathrm{O}\right)$, carbonyl fluoride $\left(\mathrm{CF}_{2} \mathrm{O}\right)$, formyl fluoride $(\mathrm{CHFO})$ and formic acid $(\mathrm{CHOOH})$. The analysis covers the entire $\mathrm{K}$ edge region from the first absorption peak to the ionization limit, i.e. including the Rydberg excitations. For the lowest energy core-excited electronic transition at both the $\mathrm{C}$ and $\mathrm{O}$ $\mathrm{K}$ edges of formaldehyde, has been performed the simulation of the associated vibrational progressions since this allows for a more direct comparison with the high-resolution experiments [219]. The vibronic calculations (of the polyatomic molecule formaldehyde) also represent a more compelling stress test for the level of theory since their quality also depend on the quality of the gradients and Hessians, and hereby constitute a more detailed investigation on the accuracy of the computational method. 


\subsection{COUPLED CLUSTER THEORY}

Coupled cluster methods are built upon the exponential ansatz of the wave function,[220]:

$$
|\Psi\rangle=e^{\hat{\top}}|0\rangle
$$

where $|0\rangle$ stands for the reference wave function and $\hat{T}=\sum_{\mu} t_{\mu} \tau_{\mu}$ is the cluster operator, which is a linear combination of the excitation operators $\tau_{\mu}$, each weighted by the corresponding CC amplitude, $t_{\mu}$. The cluster operator includes excitations established by a hierarchy of approximations given by the partion of the of the cluster operator into ranks comprising single excitations, double excitations and triple excitations. The cluster operator can be rewritten as:

$$
\hat{\mathrm{T}}=\hat{\mathrm{T}}_{1}+\hat{\mathrm{T}}_{2}+\ldots+\hat{\mathrm{T}}_{\mathrm{N}}
$$

Each excitation operators excites at least one electron from an occupied orbital to a virtual one and since there are $\mathrm{N}$ elctrons in the system, the expansion terminates at $\hat{T}_{\mathrm{N}}$. The one-electron part is given as:

$$
\hat{\mathrm{T}}_{1}=\sum_{\mathrm{AI}} \mathrm{t}_{\mathrm{I}}^{\mathrm{A}} \mathrm{a}_{\mathrm{A}}^{\dagger} \mathrm{a}_{\mathrm{I}}
$$

while the two electron part can be written as:

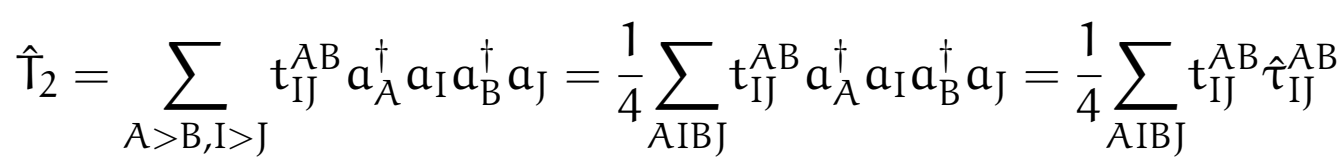

The indices I an J are used for the occupied Hartree Fock orbitals while $A$ and $B$ stand for the virtual ones. The cluster amplitudes $t_{I J}^{A B}$ are antisymmetric respect to orbital permutations [221]. At this point it can be checked that the expansion of the exponential operator $e^{\hat{T_{0}}}$ leads to:

$$
\begin{array}{r}
e^{\hat{T_{0}}}=1 \\
e^{\hat{T}_{1}}=\hat{T_{1}} \\
e^{\hat{T_{2}}}=\hat{T_{2}}+\frac{1}{2} \hat{T_{1}^{2}} \\
e^{\hat{T_{3}}=\hat{T}_{3}}+\hat{T_{1}} \hat{T_{2}}+\frac{1}{6} \hat{T_{1}^{3}}
\end{array}
$$

So excited states of a higher order also includes simultaneous excitations of lower order, for example the doubly excited states includes not only the 
connected double excitations, but also the disconnected simultaneous single excitations. This leads to the size-extensivity property of the coupled cluster, ensuring consistent results for arbitrary numbers of non-interacting molecules. The ground-state energy and CC amplitudes can be obtained by projection of the time-independent Schrödinger equation for the nonHermitian similarity transformed Hamiltonian, $\hat{\mathrm{H}}^{\hat{\top}}=e^{-\hat{\top}} \hat{\mathrm{H}} e^{\hat{\hat{T}}}$, onto the reference state $|0\rangle$ and the manifold of excited states $|\mu\rangle$

$$
\left\langle 0\left|\hat{\mathrm{H}}^{\hat{T}}\right| 0\right\rangle=\mathrm{E} ; \quad\left\langle\mu\left|\hat{\mathrm{H}}^{\hat{T}}\right| 0\right\rangle=0
$$

Within CC response theory, $[216,222]$ the CC excitation energies can be computed by determining the eigenvalues of the CC Jacobian matrix A, whose elements are $A_{\mu \nu}=\left\langle\mu\left|\left[\hat{H}^{\top}, \tau_{v}\right]\right| 0\right\rangle$.

Since the CC Jacobian is non-symmetric, the left and right eigenvectors are not each other adjoint, so two eigenvalue equations are solved

$$
\mathbf{A R}_{j}=\omega_{j} R_{j} ; \quad L_{j} \mathbf{A}=\omega_{j} L_{j}
$$

with the biorthogonality condition $L_{k} R_{j}=\delta_{j k}$. In order to solve the above large scale non-symmetric eigenvalue equations, variants of the Davidson [223] or Lanczos [224, 225] algorithms can be adopted. In the present study, the Davidson [223] algorithm is used. Transition strengths (for electric dipole moment component $\alpha$ ) can be determined as

$$
S_{0 \rightarrow j}^{\alpha \alpha}=\frac{1}{2}\left\{T_{0 j}^{\alpha} T_{j 0}^{\alpha}+\left(T_{0 j}^{\alpha} T_{j 0}^{\alpha}\right)^{*}\right\}
$$

where the left and right transition moments are given by

$$
\mathrm{T}_{0 j}^{\alpha}=\eta^{\alpha} \mathrm{R}_{j}+\bar{M}^{j}\left(\omega_{j}\right) \xi^{\alpha} ; \quad \mathrm{T}_{j 0}^{\alpha}=\mathrm{L}_{j} \xi^{\alpha}
$$

and the auxiliary Lagrangian multipliers $\bar{M}^{j}\left(\omega_{j}\right)$ are obtained from the solution of the linear equation

$$
\bar{M}^{j}\left(\mathbf{A}+\omega_{j} \mathbf{I}\right)=-\mathbf{F R}_{\mathbf{j}} .
$$

See e.g. Refs. $[222,226]$ for a definition of the $\mathbf{F}$ matrix and of the vectors $\xi^{\alpha}$ and $\eta^{\alpha}$. In order to obtain excitation energies and strengths of core-excited states, it is convenient to apply a core-valence separated scheme [227] to decouple the energetically high-lying core excited states from valence continuum states. In practice, this also avoids solving for the exceedingly large number of lower-lying valence excitations. 
Here, the projection scheme proposed in ref. [218] has been used, that enables the calculations of energies and analytic gradients with only a simple modification of a well-developed EOM-CCSD energy and gradient code. A projector $\mathcal{P}_{\mathrm{I}}^{v}$ is applied at each iteration in the iterative solvers to remove all vector elements not referencing at least one core orbital (or a set of selected core orbitals) I, e.g. in the Davidson case,

$$
\mathcal{P}_{I}^{v}\left(\mathbf{A P}_{I}^{v} R_{j}\right)=\omega_{j} \mathcal{P}_{I}^{v} R_{j}
$$

and similarly for the left eigenvectors. Further details can be found in ref. [218]. We should mention that the equations that determine the Lagrange multipliers for the cluster amplitudes correlate all electrons, to be consistent with the all-electron CC treatment for the ground state. This proved to be particularly important during the determination of the multipliers for the core excited state gradient, to be able to reproduce the results of a finite difference determination of the gradient. In the Born-Oppenheimer approximation, vibronic spectra arise from the contribution of the transitions from all populated vibrational states in the initial electronic state, $\left|\boldsymbol{v}_{1}\right\rangle$, to those in the final electronic state, $\left|\boldsymbol{v}_{2}\right\rangle$. The spectrum as function of the radiation frequency, $\omega$, takes the following form: [228]

$$
\begin{aligned}
\mathrm{S}(\omega)= & \mathrm{C} \omega \sum_{v_{1}, v_{2}} \rho_{v_{1}}(\mathrm{~T})\left(\left|\left\langle\boldsymbol{v}_{1}\left|\boldsymbol{\mu}_{12}\right| \boldsymbol{v}_{2}\right\rangle\right|^{2}\right) \\
& \times \delta\left(\omega_{v_{2}}-\omega_{v_{1}}+\left(\hbar^{-1} \Delta \mathrm{E}-\omega\right)\right)
\end{aligned}
$$

here $\rho_{v_{1}}$ are the Boltzmann weights associated to each vibrational level in the initial electronic state, $\mu_{12}$ is the electronic transition dipole moment connecting both electronic states, $\omega_{v_{1}}$ and $\omega_{v_{2}}$ are the frequencies of each vibrational state on either the initial or final electronic states and $\Delta \mathrm{E}$ is the energy difference between the minima of each state (adiabatic energy). $C$ is a constant whose value is $\sim 703.3$ when all quantities are in atomic units in order to provide the spectrum as molar extinction coefficient $(S(\omega) \equiv \varepsilon(\omega))$ in the usual experimental units: $\mathrm{M}^{-1} \mathrm{~cm}^{-1}$. The above Time-Independent (TI) expression implies a sum-over-states that can become unfeasible for large systems and/or high temperatures [228]. In these cases, the spectrum can be obtained more efficiently from an alternative, Time-Dependent (TD), formulation derived by taking the Fourier transform of the Dirac delta function, [229] which leads to

$$
S(\omega)=\frac{C \omega}{2 \pi Z_{v_{1}}} \int \chi(t, T) e^{-i t(\Delta E / \hbar-\omega)} d t
$$


where $Z_{v_{1}}$ is the vibrational partition function of the initial states, $\left|\boldsymbol{v}_{1}\right\rangle$, and $\chi(t, T)$ is the correlation function, given by

$$
\chi(t, T)=\operatorname{Tr}\left[\mu_{12} e^{-i t H_{2} / \hbar} \mu_{12} e^{-(\beta-i t) H_{1} / \hbar}\right]
$$

where Tr refers to the trace operation, $\beta=\left(K_{B} T\right)^{-1}, K_{B}$ is the Boltzmann constant, $\mathrm{T}$ the absolute temperature, and $\mathrm{H}_{1}$ and $\mathrm{H}_{2}$ are the initial-state and final-state Hamiltonians respectively.

\subsubsection{Computational Details}

Four different systems have been considered: formaldehyde $\mathrm{CH}_{2} \mathrm{O}$, formic acid $\mathrm{CHOOH}$, carbonyl fluoride $\mathrm{CF}_{2} \mathrm{O}$, and formyl fluoride $\mathrm{CHFO}$. Experimental geometries from refs. [230-233] have been used for all molecules. The hierarchy CCS (coupled-cluster singles), CC2 (coupled cluster singles and approximate doubles) and CCSD (coupled cluster singles and doubles) with the eCVS scheme, [218] available in the Dalton code, [234] has been used to compute both core excitation energies, intensities and ionization potentials.

Different correlation consistent basis sets, [235] further enriched with Rydbergtype basis functions, [236] were used. For formaldehyde, two sets of Rydberg functions were tested, namely a $3 \mathrm{~s} 3 \mathrm{p} 3 \mathrm{~d}$ set (with quantum number $\mathrm{n}=3-$ 4 ), and a $7 \mathrm{~s} 7 \mathrm{p}$ set (with $n=2-5$ ), both obtained according to Kaufmann's prescription. [236] The notation $(3 \mathrm{~s} 3 p 3 d)_{\mathfrak{n}=3-4}(7 s 7 p)_{\mathfrak{n}=2-5}$ will be used in the follwowing when referring to such sets.

In all the plots shown in the next sessions, a rigid shift has been applied in order to align each simulated spectrum with the experimental one. The shift was determined from the energy difference $\Delta$ between the first computed and the first experimental peak. For the vibrational structure, optimized structures for both ground and core-excited states were obtained at the (EOM-)CCSD/aug-cc-pCVTZ level using the same eCVS scheme, implemented in CFOUR [237]. The ground and excited state Hessians were also obtained, using a fully analytical approach for the ground state [238-241] and by using numerical differentiation of analytical gradient $[242,243]$ for the excited states.

Vibronic spectra have been obtained adopting the harmonic approximation. For the vibrational analysis, the potential energy surfaces (PES) of the ground state and the core excited states have been modeled with a quadratic expansion around their minima (i.e. with the so-called adiabatic Hessian approach, $\mathrm{AH}[244])$. Moreover, the absorption spectra have been computed 
in Franck-Condon approximation (i.e., the electronic transition dipole moment $\mu_{12}$ was assumed independent of nuclear coordinates), adopting both the time-independent [228] and the time-dependent [229] methods implemented in version 3.0 of the FCclasses code [245].

\subsection{FORMALDEHYDE}

In order to determine the basis set requirements, we considered in Figure. 20, the oxygen and carbon $\mathrm{K}$ edge spectra of $\mathrm{CH}_{2} \mathrm{O}$ in comparison with the experimental spectra. [219] The main spectral features for $\mathrm{CH}_{2} \mathrm{O}$ at both the carbon and oxygen $\mathrm{K}$ edge is the $1 \mathrm{~s} \rightarrow \pi^{*}$ transition, reported in the literature at $285.59 \mathrm{eV}$ and $530.82 \mathrm{eV}$, respectively. They are followed, in the higher energy part of the the spectra, by a series of Rydberg transitions [219]. The family of correlation consistent basis sets [246] (cc-pVXZ) has been widely employed in accurate ab-initio calculations because of their systematic convergence of the calculated properties towards the complete basis set limit when increasing the cardinal number X. [247]

Since a large fraction of the correlation energy needs to be recovered, one may expect that large basis sets are needed, [248] at least of triple zeta quality.

The relaxation of the core is addressed by polarization and core-correlating functions. One can also expect that the description of excited states of diffuse character will benefit from the inclusion of augmentation functions, and that the addition of center-of-mass Rydberg-type functions in particular will allow a better description of the Rydberg region [236].

Using the above prescriptions, the cardinal number of basis set was varied as $\mathrm{X}=\mathrm{D}, \mathrm{T}, \mathrm{Q}$, and, in Figure 20, the oxygen and carbon $\mathrm{K}$ edge spectra of $\mathrm{CH}_{2} \mathrm{O}$ obtained at the CCSD level with different basis sets are shown. The spectra were generated by applying a Lorentzian lineshape with $\mathrm{HWHM}=0.2 \mathrm{eV}$ to the electronic stick-transitions. The oxygen $\mathrm{K}$ edge spectra in the double $\zeta$ basis shows a positive deviation, $\Delta$, of $3.68 \mathrm{eV}$ (i.e., the core excitation energy of the reference peak is overestimated), while the triple and quadruple $\zeta$ sets have deviations of $1.72 \mathrm{eV}$ and $1.59 \mathrm{eV}$, respectively. The computed spectra were therefore shifted by $-\Delta$. The spectral shape is less affected than the absolute energy by the basis set, so that even a small basis set can reasonably reproduce the experimental features.

In Figure 20, the energy separations between the $1 s \rightarrow \pi^{*}$ and the first Rydberg transition in the oxygen $\mathrm{K}$ edge are also reported, and compared to the experimental reference value of $4.61 \mathrm{eV}$. The peak separations are less 

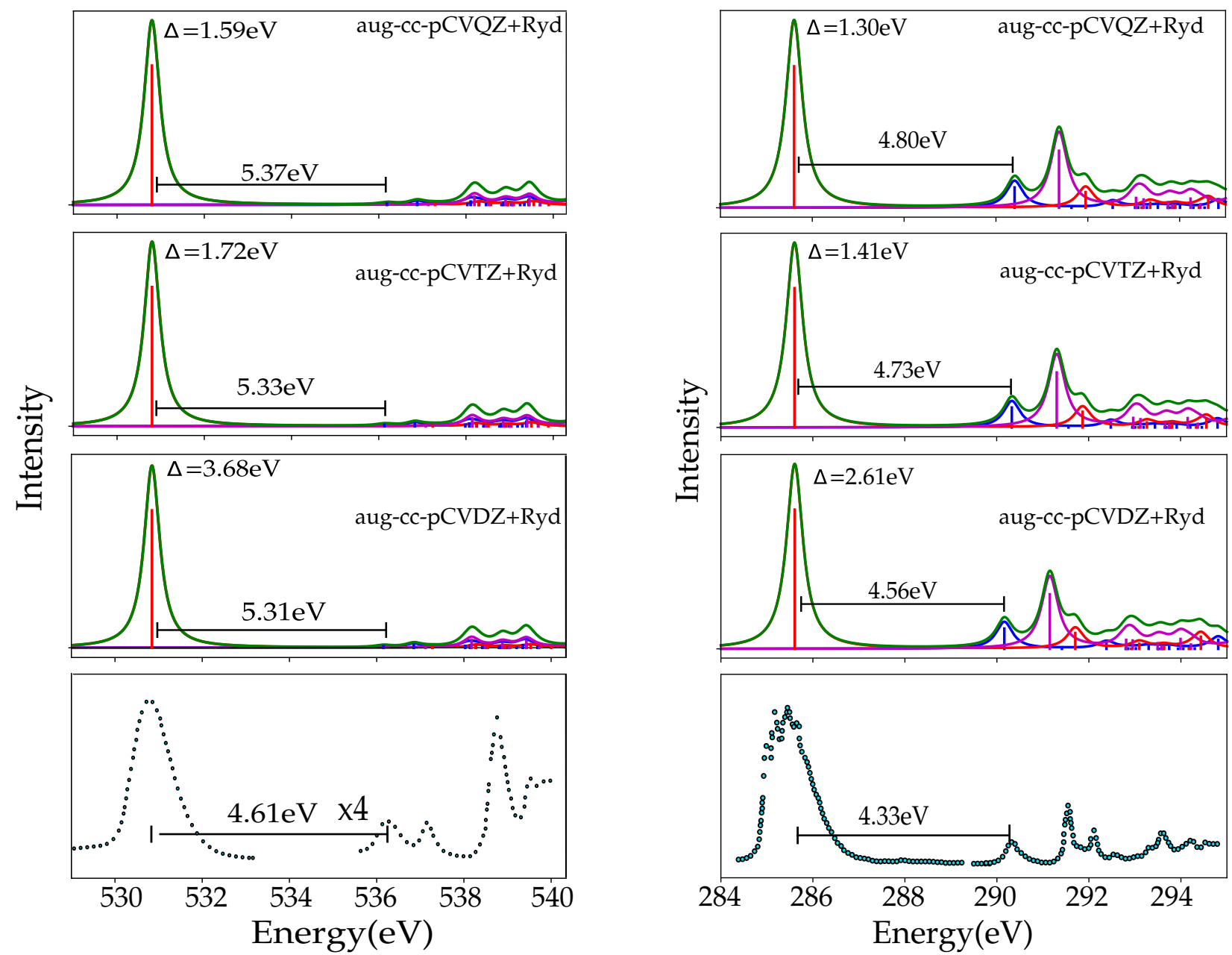

Figure 20: Formaldehyde: eCVS-CCSD oxygen (left) and carbon (right) K edge spectra in different correlation consistent basis sets supplemented with (3s3p3d) Rydberg functions. A parallel shift $-\Delta$ was applied to the computed spectra to align them with the first experimental peak. The total spectra are reported in green; blue lines and sticks refer to transitions of $A_{1}$ symmetry, red lines to those of $B_{1}$ symmetry and magenta lines to those of $B_{2}$ symmetry, for the molecule placed on the $y z$ plane and the $\mathrm{C}_{2}$ axis along $z$. A Lorentzian broadening of $\mathrm{HWHM}=0.2 \mathrm{eV}$ has been applied. The experimental spectra are taken from ref. [219]. The spectral profiles shown have been generated by broadening the raw spectral data reported in Table 6 and Table 7 of the Appendix. 
affected than the absolute energy by the variation in cardinal number, and they are overestimated by approx. $0.6 \mathrm{eV}$.

We expect that this somewhat large separation is reduced if triple and higher excitations are taken into account, which would be of interest for future investigations. In the right panels of Figure 20, the same basis sets used for the oxygen $\mathrm{K}$ edge are tested for the carbon $\mathrm{K}$ edge. The high-resolution experimental spectrum [219] shows a rich vibrational progression that will be discussed later on. Also in this case, the double $\zeta$ basis set shows the larger shift with respect to the reference experimental value $(2.61 \mathrm{eV})$, and the overall shift is smaller than in the oxygen case.

This appears to be a general trend, as revealed by inspection of the results for the $1 \mathrm{~s} \rightarrow \pi^{\star}$ excitation of all molecules here considered, collected in Table 17: the higher the energy to excite the core electron is, the larger is the shift of the computed peak from the experimental counterpart. In the case of $\mathrm{CH}_{2} \mathrm{O}$, both edges show a small difference in the absolute energy shift between the triple and the quadruple $\zeta$ basis set.

As the overall spectral shape before the ionization limits $(294.3 \mathrm{eV}$ for the carbon and 539.3 $\mathrm{eV}$ for the oxygen threshold [219]) are not largely affected by the size of the basis set, one can possibly use a basis set of double- $\zeta$ quality if the results are then shifted accordingly. It should be noted, however, that this is partly due to the fact that all basis sets considered above include additional Rydberg functions, which play a fundamental role in the correct description of the weak bands in the spectra. This is clearly evident in Figure 21 , where the spectra obtained at both carbon and oxygen $\mathrm{K}$ edges using the core-valence triple $\zeta$ basis with and without Rydberg and augmentation functions are compared.

While the position of the $1 s \rightarrow \pi^{*}$ peak is roughly the same for all three basis sets, a correct description of the Rydberg region clearly requires the inclusion of diffuse functions (sufficient to describe the lower lying Rydberg states) and of Rydberg-type functions (for the higher lying Rydberg states).

In Figure 22,the spectra obtained for $\mathrm{CH}_{2} \mathrm{O}$ using triple $\zeta$ basis sets with and without core polarization and/or augmentation functions, combined with two different choices of Rydberg-type basis sets are shown.

At both edges, the smallest systematic shift is found for the aug-cc-pVTZ basis set, whereas in the core-valence bases with and without augmenting functions the shifts are roughly the same. The two selected Rydberg-type basis sets yield similar spectral profiles at frequencies above the $1 \mathrm{~s} \rightarrow \pi^{*}$ peak, though with some differences in the intensities and in the separation from the $1 \mathrm{~s} \rightarrow \pi^{*}$ band, in particular when combined with the cc-pCVTZ basis set. 

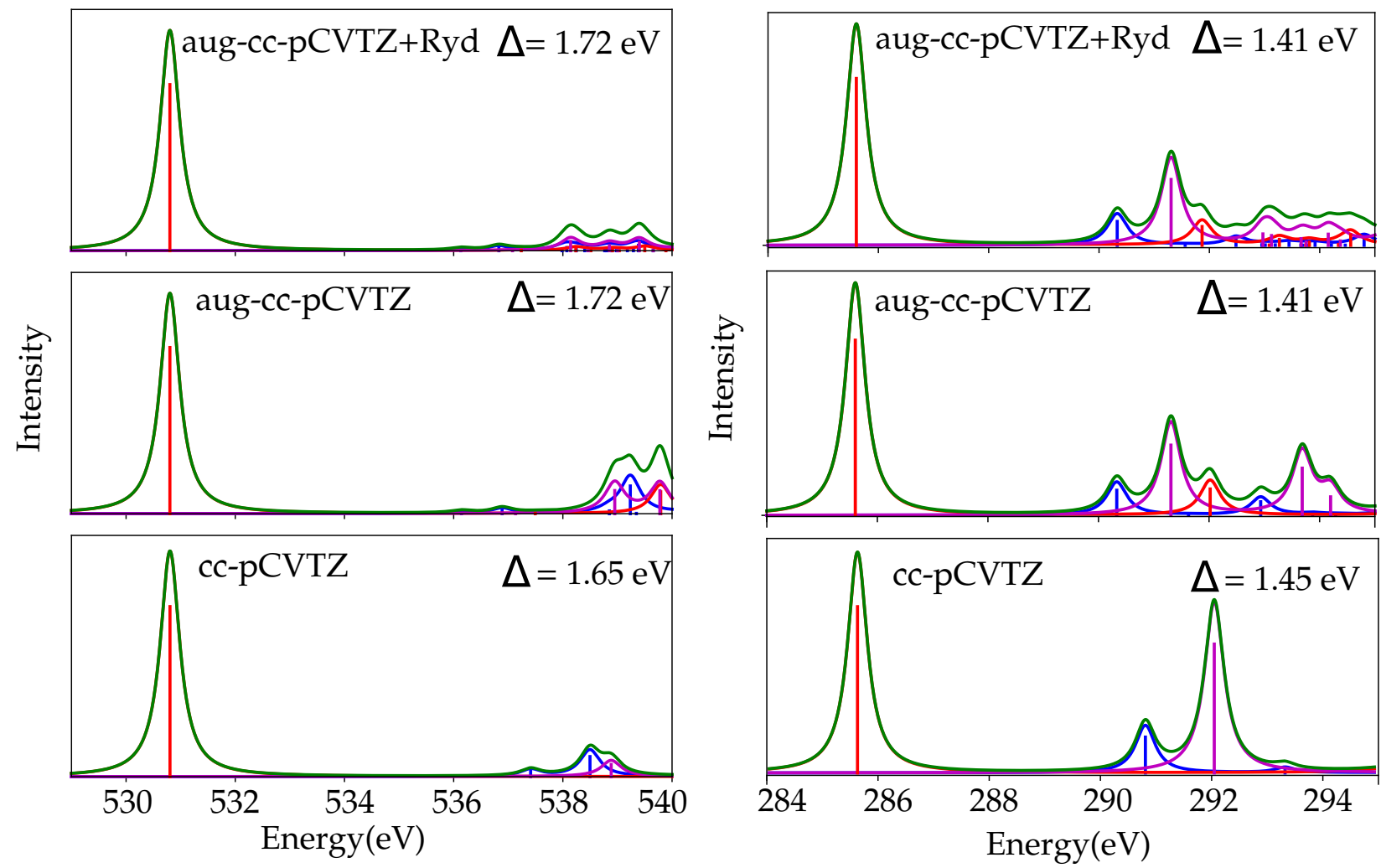

Figure 21: Formaldehyde: eCVS-CCSD carbon (left) and oxygen (right) K edge spectra using the cc-pCVTZ basis, the aug-cc-pCVTZ and the aug-cc-pCVTZ basis plus the (3s3p3d) Rydberg set. The parallel shifts $-\Delta$ applied to the computed spectra to align them with the first experimental peak are indicated. The total spectra are reported in green; blue lines and sticks refer to transitions of $A_{1}$ symmetry, red lines to those of $B_{1}$ symmetry and magenta lines to those of $B_{2}$ symmetry, for the molecule placed on the $y z$ plane and the $C_{2}$ axis along $z$. A Lorentzian broadening of $\mathrm{HWHM}=0.2 \mathrm{eV}$ has been applied. 
Depending on the basis set adopted, the separation between the first peak in the Rydberg region and the strong $1 \mathrm{~s} \rightarrow \pi^{*}$ band is overestimated by approximately $0.6-0.9 \mathrm{eV}$ in the case of oxygen and by ca. $0.3^{-0.6 \mathrm{eV}}$ in the case of carbon. It can be noted that, since core electrons are involved, one can expect relativistic effects to play a role in the energetic of the excitation. The inclusion of the relativistic effects by means of the Douglas-Kroll-Hess scalar relativistic Hamiltonian [249, 250] and by the spin-free exact two-component theory in its one-electron variant $[251,252]$ has been shown to give a small (positive) shift in light elements, [225, 253-256] consistent with the relativistic contraction of inner orbitals.

Having analyzed, for $\mathrm{CH}_{2} \mathrm{O}$, the role of the basis set at the CCSD level, we now turn our attention to the importance of the accurate description of electron correlation within the CC hierarchy and show, in Figure 23, the spectral profiles obtained at the $\mathrm{O}$ and $\mathrm{C}$ edges of formaldehyde within the eCVSCCS, eCVS-CC2 and eCVS-CCSD hierarchy in the aug-cc-pCVTZ basis set supplemented with the $(3 \mathrm{~s} 3 \mathrm{p} 3 \mathrm{~d})_{\mathrm{n}=3-4}$ Rydberg basis functions.

The computed spectra are once again compared with the experimental counterparts from ref. [219]. At the CCS level of theory (which is equivalent to configuration interaction singles, CIS), the position of the XAS first band is overestimated by as much as $15 \mathrm{eV}$ at the $\mathrm{O}$ edge and $8.7 \mathrm{eV}$ at the $\mathrm{C}$ edge. The next band is found at $\approx 9.8 \mathrm{eV}$ for oxygen and $\approx 8.07 \mathrm{eV}$ for carbon, respectively, and its intensity, relative to the first band, is too large.

It is thus clear that CCS does not give a satisfactory description of the energetics of the excitations, of their relative intensities and therefore of the overall spectral features.

The inadequacy of the CCS method is mainly due to the inability of the method to describe the strong orbital relaxation effects that follow the excitation of core electrons, due to the complete lack of double excitations in both ground and excited state descriptions.

Indeed, when orbital relaxation is explicitly taken into account, for instance by using as basis the electron-attached states of independently optimized, core-ionized references, (non-orthogonal) CIS-based methods have been shown to yield good agreement with experiment, at least for the $1 \mathrm{~s} \rightarrow \pi^{*}$ excitations. [257, 258] In CC2, the (ground state) doubles amplitudes are correct to first order and the singles to second order. The single-single block of the CC2 Jacobian is correct to second order, whereas the double-single and single-double blocks are correct to first order, since the lowest-order coupling to the singles spectrum is retained. This ensures that the CC2 single replacement dominated excitation energies are correct through second 

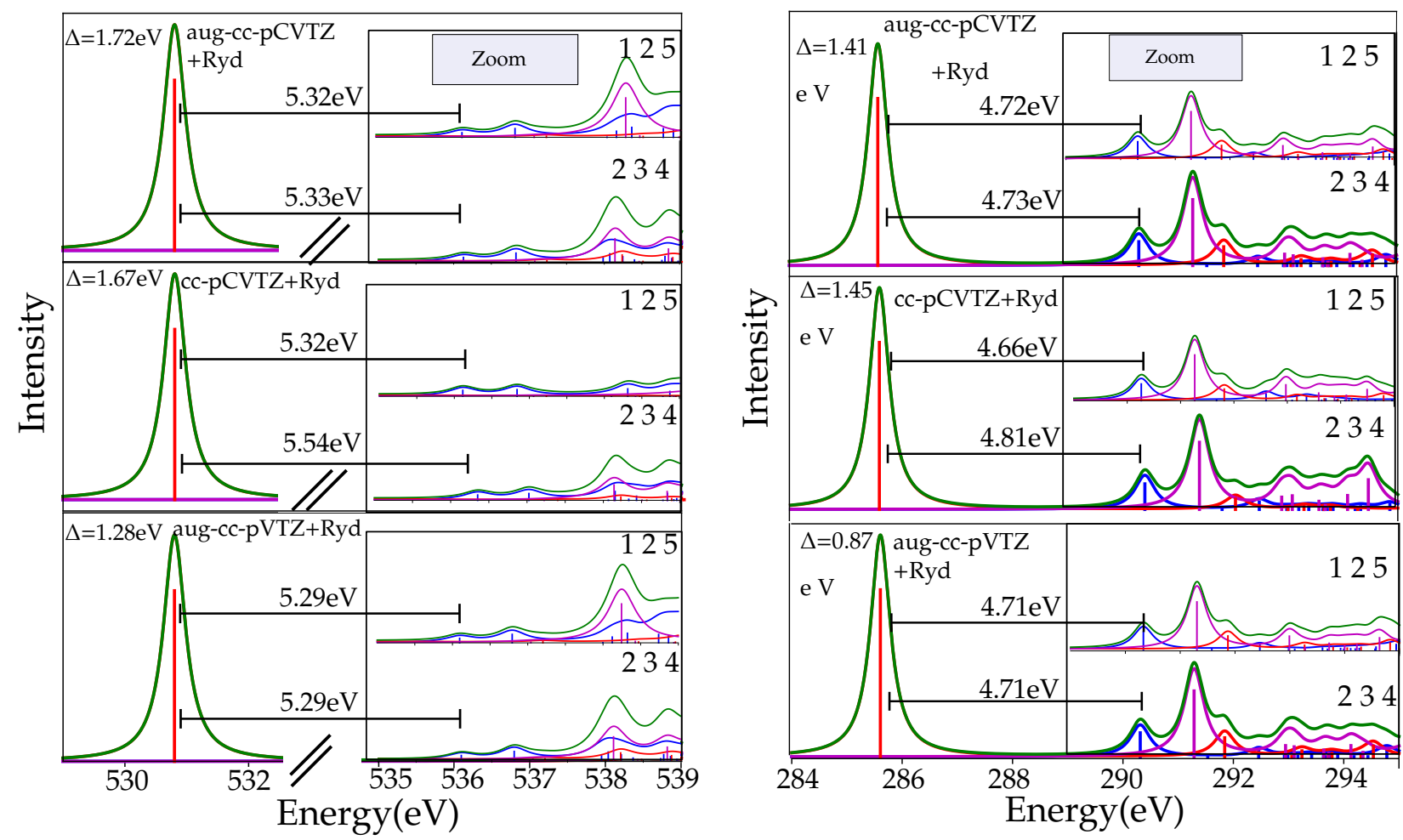

Figure 22: Formaldehyde: eCVS-CCSD oxygen (left) and carbon (right) K edge using $\zeta$ zeta basis sets with both augmentation and core correlation functions, with core correlation functions only, and with augmentation functions only. Two different Rydberg spaces are reported. A parallel shift $-\Delta$ was applied to align the computed spectra with the first experimental peak. The reported value between the first and the second peak is the difference in energy between these two features. A Lorentzian broadening of HWHM=0.2 eV has been applied.

The total spectra are in green; blue lines and sticks refer to transitions of $A_{1}$ symmetry, red lines to those of $B_{1}$ symmetry and magenta lines to those of $B_{2}$ symmetry, for the molecule placed on the $y z$ plane and the $\mathrm{C}_{2}$ axis along $z$. 

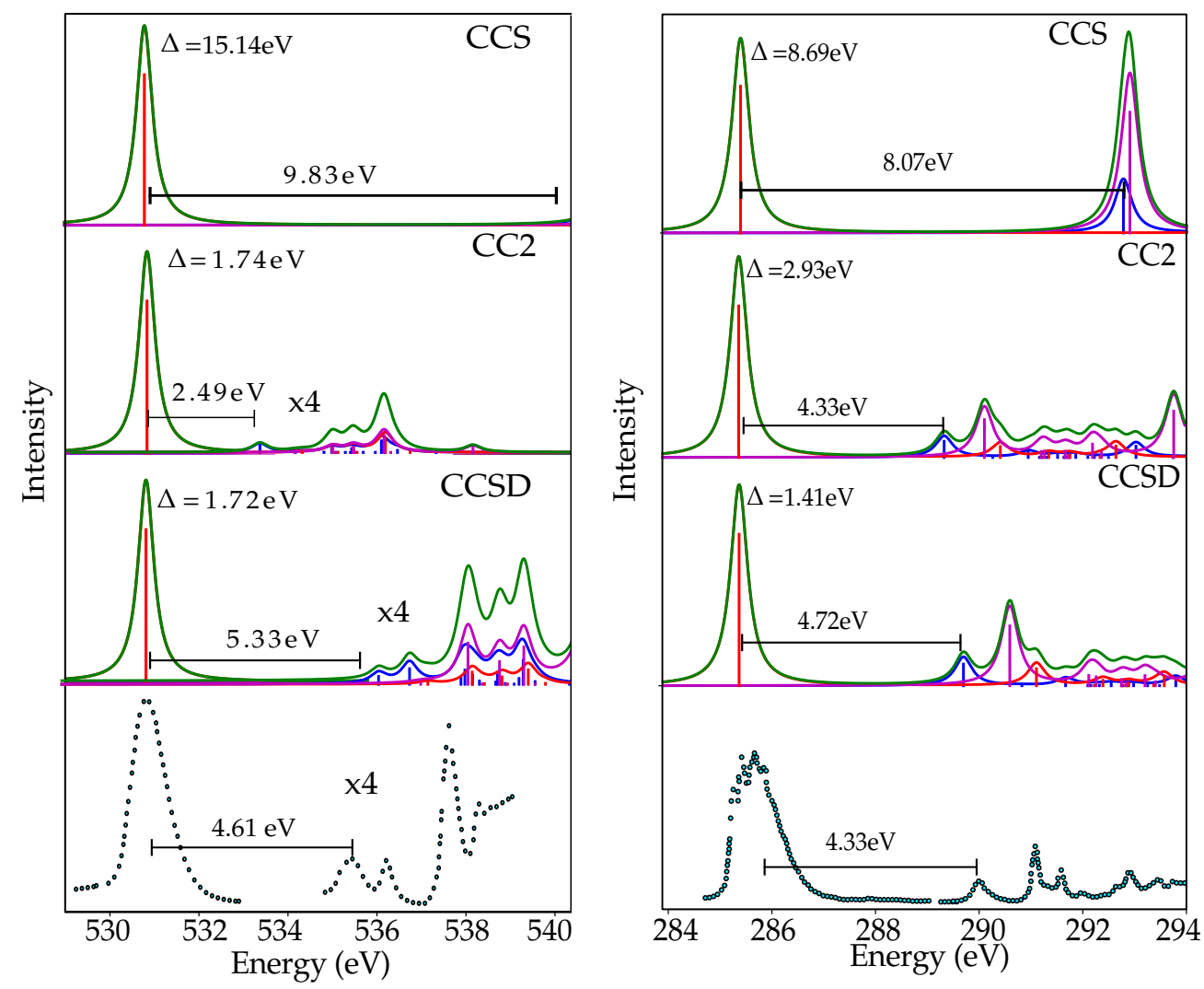

Figure 23: Formaldehyde: eCVS-CCS, eCVS-CC2 and eCVS-CCSD oxygen (left) and carbon (right) $\mathrm{K}$ edge spectra in the aug-cc-pCVTZ basis set supplemented with Rydberg functions, compared with the experimental results from ref. [219]. The total spectra are in green; blue lines and sticks refer to transitions of $A_{1}$ symmetry, red lines to those of $B_{1}$ symmetry and magenta lines to those of $B_{2}$ symmetry, for the molecule placed on the $y z$ plane and the $C_{2}$ axis along $z$.The spectral profiles shown have been generated by broadening the raw spectral data reported in Table 6, 8 and 9 of the Appendix. 
order. Double replacement dominated excitations, on the other hand, cannot be described by CC2 [259, 260]. The CC2 oxygen K edge spectrum of formaldehyde in Figure 23 shows a shift of around $1.7 \mathrm{eV}$, which is basically the same as in the CCSD case. It was observed earlier [224] that CC2 can sometimes give better absolute core energies than CCSD, but this can be related to some error cancellation effects between the incomplete treatment of the double excitations and the lack of triple excitations [225]. Despite the good alignment of the first excitation energy, however, the remaining peaks in the eCVS-CC 2 oxygen $\mathrm{K}$ edge spectrum are too close, the second peak being located at only $\approx 2.5 \mathrm{eV}$ from the first one.

At the carbon K edge, on the other hand, the rigid shift of the CC2 spectrum with respect to the experimental data is $2.93 \mathrm{eV}$, almost twice the value of $1.41 \mathrm{eV}$ found for CCSD. Thus, at the carbon K edge edge, the overall spectral profiles yielded by both CC2 and CCSD match rather well the experimental one, with a separation between the first and second peak reasonably close to the (estimated) experimental value.

Turning the attention to the ionisation energies (IE) and (relative) term values $(\mathrm{T})$ reported in Table 17 for formaldehyde, the eCVS-CCSD core ionisation energy of $\mathrm{C}$ is $\approx 1.5 \mathrm{eV}$ higher than experiment, which yields a slightly overestimated term value ( $9.05 \mathrm{eV} v$ s the experimental value of $8.93 \mathrm{eV})$. The eCVS-CC2 core ionisation energy is almost the same, but the resulting $\mathrm{T}$ is underestimated $(7 \cdot 5 \mathrm{eV})$. The computed oxygen $\mathrm{K}$ edge ionisation energy at the CCSD level yield a term value for the first excitation of $9.16 \mathrm{eV}$, versus the experimental value of $9 \mathrm{eV}$. The term value is, on the other hand, underestimated by $3.5 \mathrm{eV}$ at the $\mathrm{CC} 2$ level, mainly because of the underestimation of the core ionisation energy at this level of theory. The core ionisation energies and term values at both edges are strongly overestimated by eCVS-CCS. To conclude the analysis of the purely electronic XAS spectra of formaldehyde, in Figures 24 and 25 is reported a characterization of the XAS bands obtained at the eCVS-CCSD/aug-cc-pCVTZ+(3s3p3d $)_{n=3-4}$ Rydberg level by means of the natural transition orbitals (NTO) for the underlying core excitations. The assignments originally done by [219] are also indicated in the figures. 


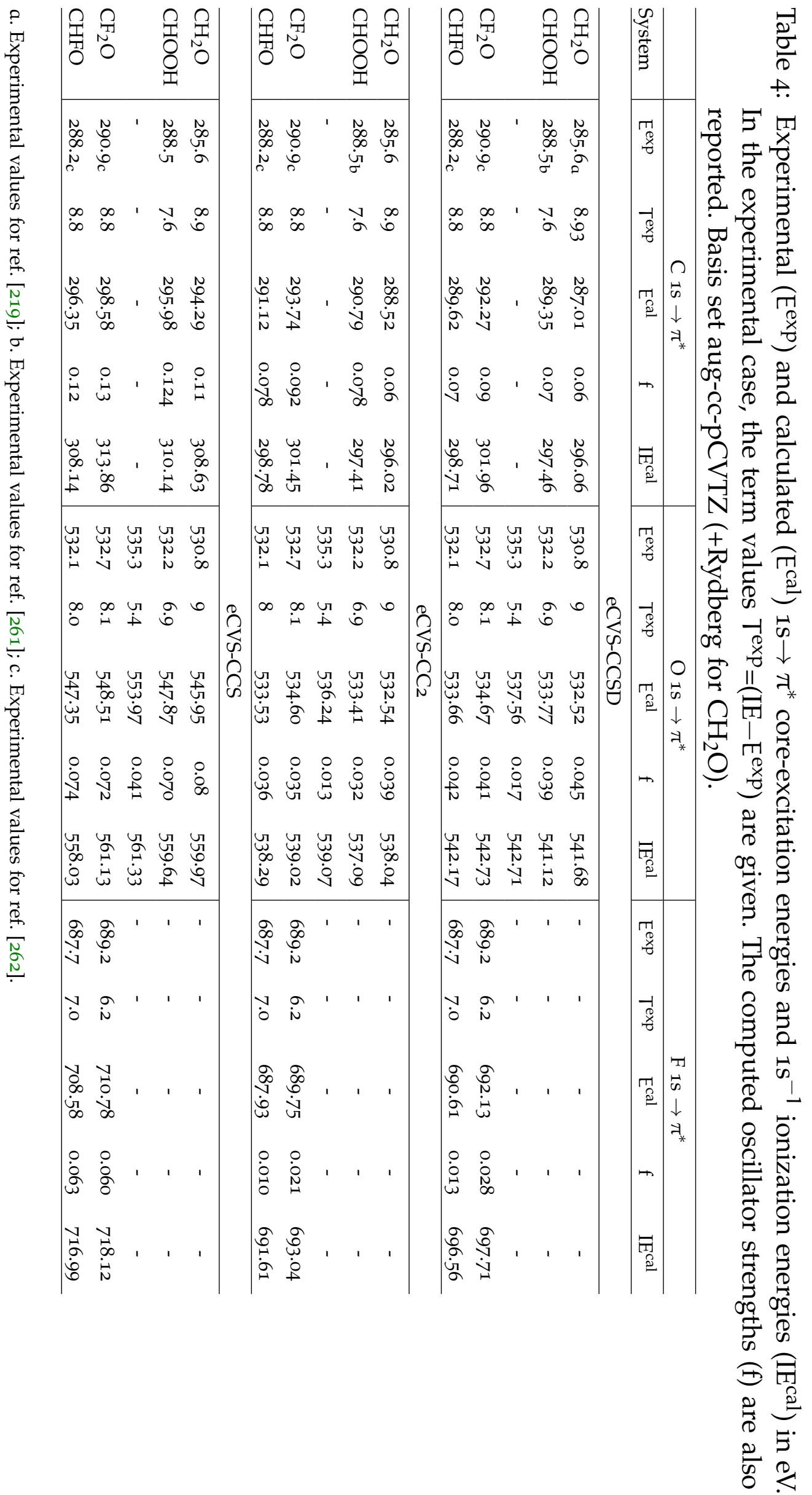




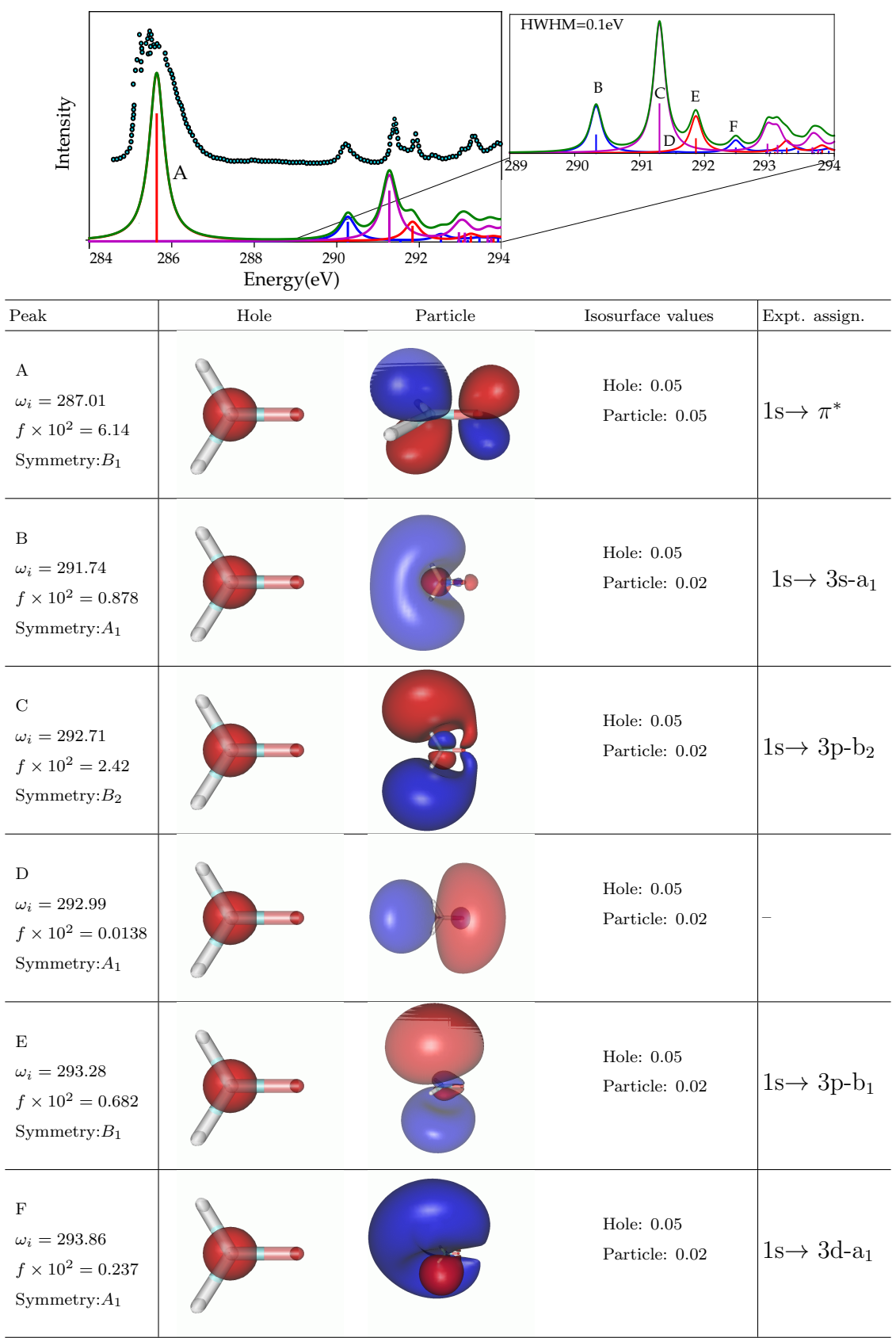

Figure 24: Formaldehyde: eCVS-CCSD NTOs of the lowest C K edge excitations. The computed spectrum has been shifted to align with experiment [219]. 


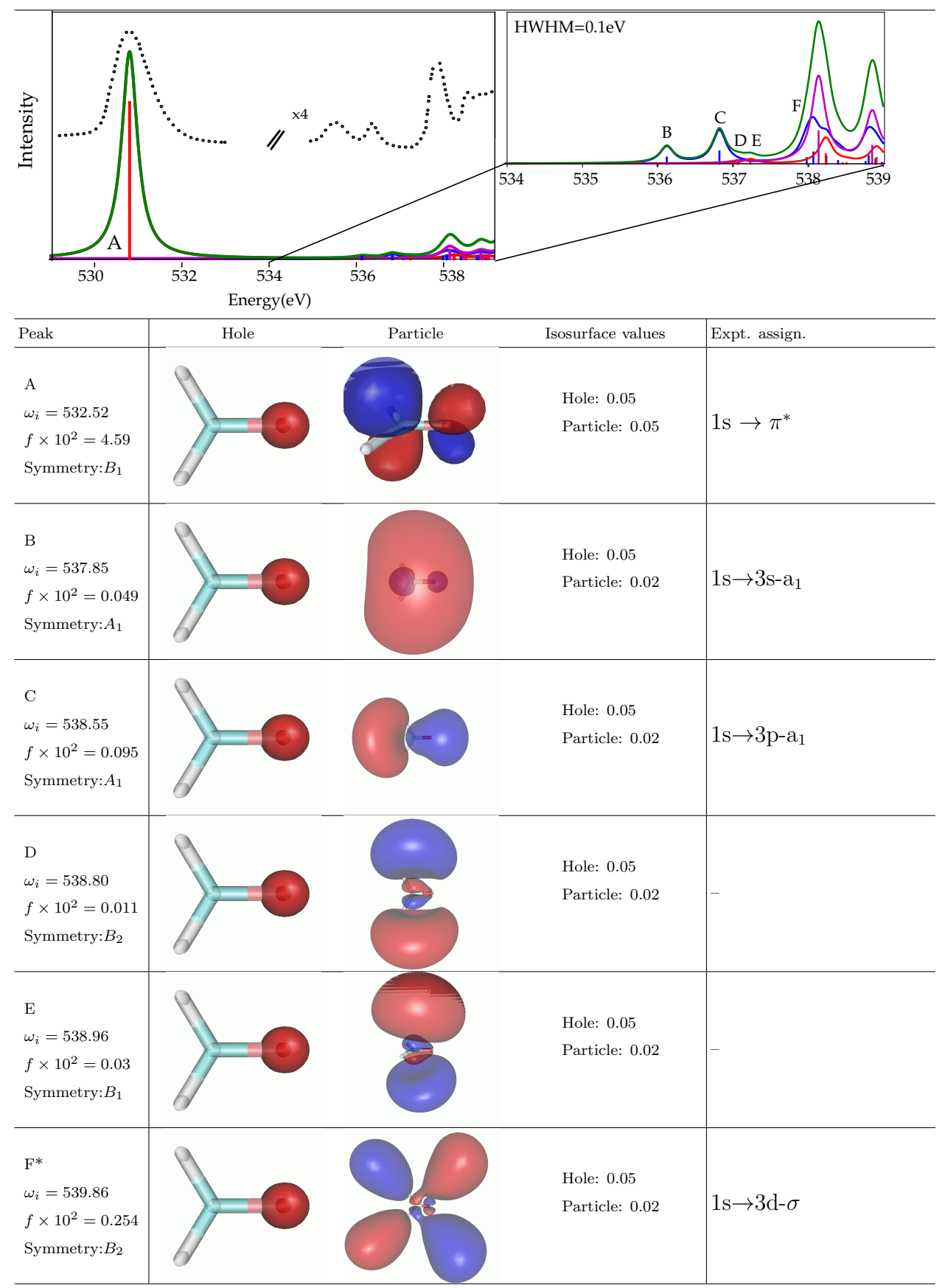

Figure 25: Formaldehyde: eCVS-CCSD NTOs of the lowest oxygen K edge excitations. For the $\mathrm{F}$ band, only the NTO of the dominant $\mathrm{B}_{2}$ component is reported. The computed spectrum has been shifted to align with experiment [219]. 
For each transition, the dominant hole/particle pair is indicated. The symmetry labelling indicated for each transition is the one resulting from the calculations [219]. The assignments of the peaks are mostly in line with the ones of [219], [219] (and [263] [263]).

\subsection{FORMIC ACID, FORMYL FLUORIDE AND CARBONYL FLUORIDE}

Having discussed in greater detail both basis set and correlation effects in the case of the formaldehyde molecule in the previous section, here are briefly considered the other three molecular systems, for which experimental data are either more scarce or less resolved.

As anticipated, in Table 17 have been collected, the eCVS-CCS, eCVS-CC2 and eCVS-CCSD results for the $1 \mathrm{~s} \rightarrow \pi^{*}$ excitations, oscillator strengths, and core-ionization energies of all edges in formaldehyde, carbonyl fluoride, formyl fluoride and formic acid obtained in the aug-cc-pCVTZ basis set (plus Rydberg for formaldehyde). Experimental absolute energies and term values $\mathrm{T}$ are also included. The full spectral profiles yielded by the three coupled cluster methods for $\mathrm{CHOOH}, \mathrm{CHFO}$ and $\mathrm{CF}_{2} \mathrm{O}$ are reported in Figures 26 to Figure 28.

At CCS level of theory, the deviation from experiment of the absolute energy of the first excitation at the $\mathrm{C}$ edges varies in between 7 and $16 \mathrm{eV}$, depending on the molecules; for the oxygen edge, it is around $15^{-16} \mathrm{eV}$ and for fluorine it is of the order of $21 \mathrm{eV}$.

From Table 17 and Figures 26-27, it is again clear that CCS completely fails in describing both the energetics and the overall spectral features for all systems, due to its inability to describe core orbital relaxation. In the case of formic acid, see Figure 26, the eCVS-CC2 systematic shift $\Delta$ is smaller than the eCVS-CCSD one at the oxygen $\mathrm{K}$ edge, but more than twice as large at the carbon $\mathrm{K}$ edge. The eCVS-CC2 spectrum at the oxygen $\mathrm{K}$ edge is again more compressed than the eCVS-CCSD one, whereas at the carbon K edge the eCVS-CC 2 and eCVS-CCSD spectral profiles are very similar, at least up to $294 \mathrm{eV}$.

Figures 27 and 28 show the XAS spectra at the carbon, oxygen and fluorine $\mathrm{K}$ edges in formyl fluoride and carbonyl fluoride, respectively, obtained with the eCVS-CCS, eCVS-CC2 and eCVS-CCSD hierarchy and the aug-ccpCVTZ basis set. As in the previous cases, eCVS-CC2 yields spectral profiles very similar to eCVS-CCSD at the carbon $\mathrm{K}$ edge, whereas at the oxygen $\mathrm{K}$ edge the peak separations are underestimated and the spectra appear com- 


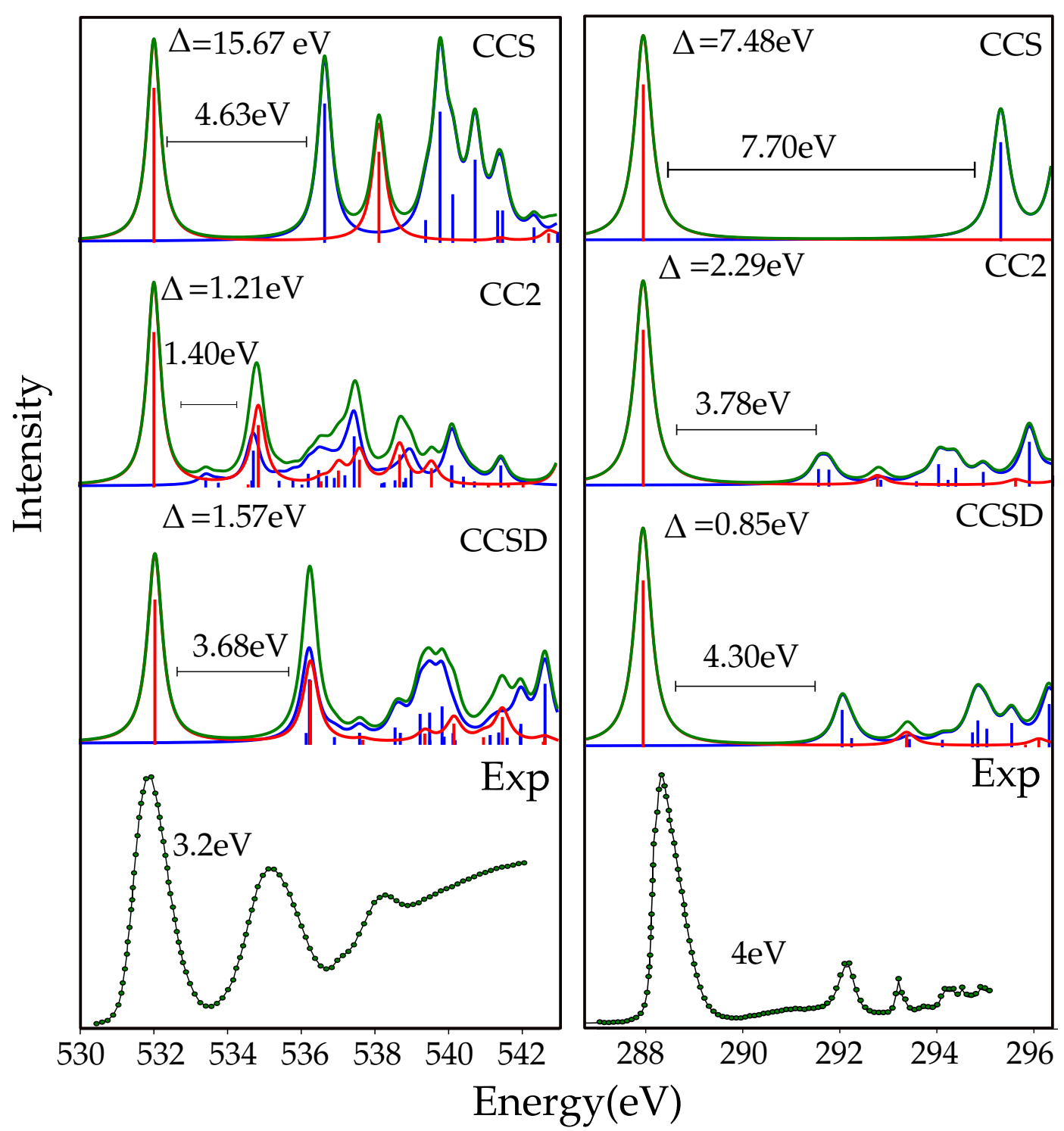

Figure 26: Formic acid: eCVS-CCS, eCVS-CC2 and eCVS-CCSD oxygen (left) and carbon (right) $\mathrm{K}$ edge spectra using, respectively, triple $\zeta$ basis sets with augmentation and core correlation functions, without Rydberg functions. The computed spectra were shifted by $-\Delta$ in order to align them with the first experimental peak. The experimental data are taken from ref. [261].The total spectra are in green; blue lines and sticks refer to transitions of $A^{\prime}$ symmetry and red lines to those of $A^{\prime \prime}$ symmetry for the molecule with $C_{s}$ symmetry. The spectral profiles shown have been generated by broadening the raw spectral data reported in Table 14 and 15 of the Appendix 

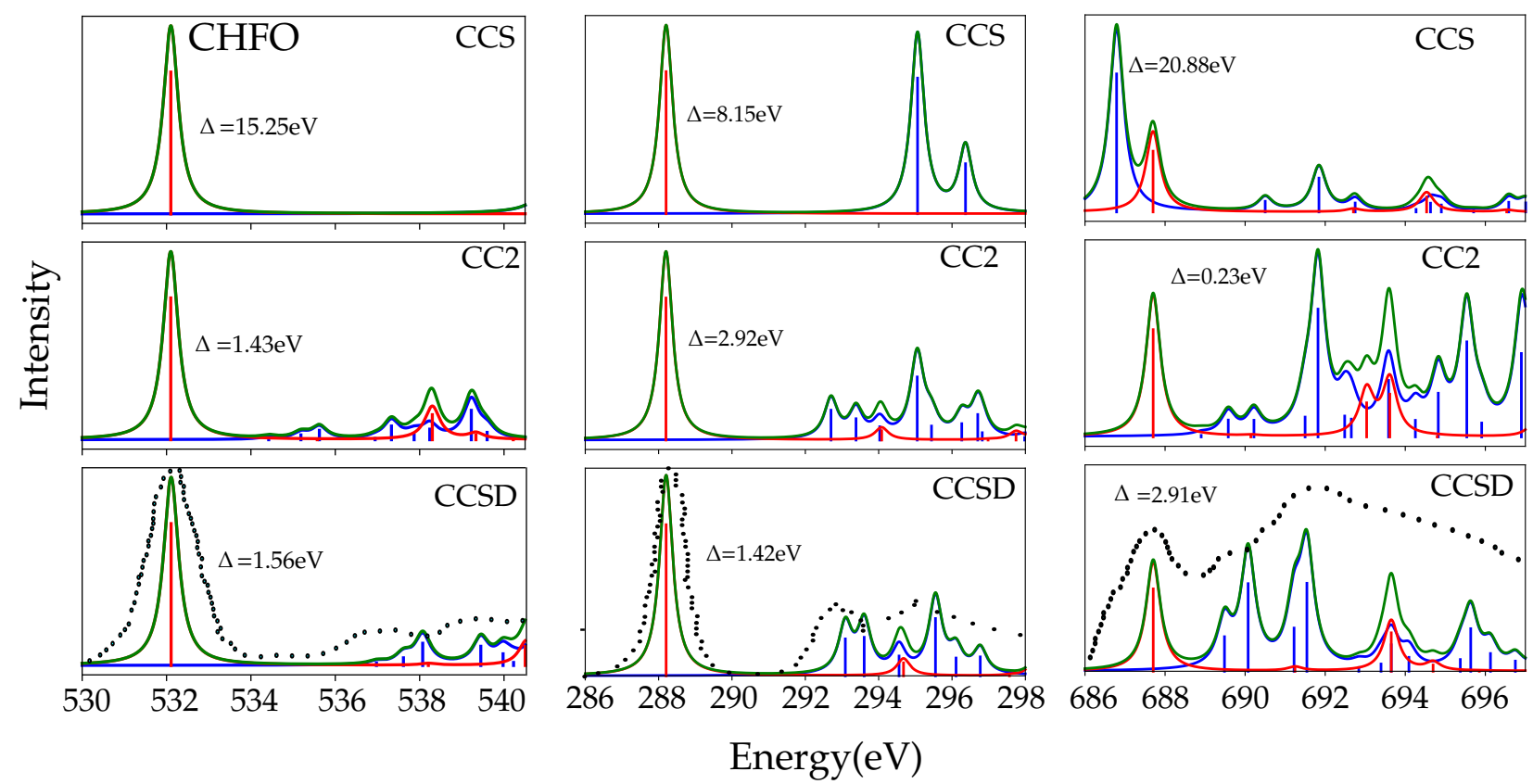

Figure 27: CHFO: eCVS-CCSD, -CC2 and -CCS carbon, oxygen and fluorine K edge spectra using the aug-cc-pCVTZ basis set. The symbol $\Delta$ indicates the shift applied to align the computed spectra with the first experimental peak. The experimental data are taken from ref. [264]. The total spectra are in green; blue lines and sticks refer to transitions of $\mathrm{A}^{\prime}$ symmetry and red lines to those of $\mathrm{A}^{\prime \prime}$ symmetry for the molecule with $\mathrm{C}_{s}$ symmetry.The spectral profiles shown have been generated by broadening the raw spectral data reported in Table 10 and 11 of the Appendix. 

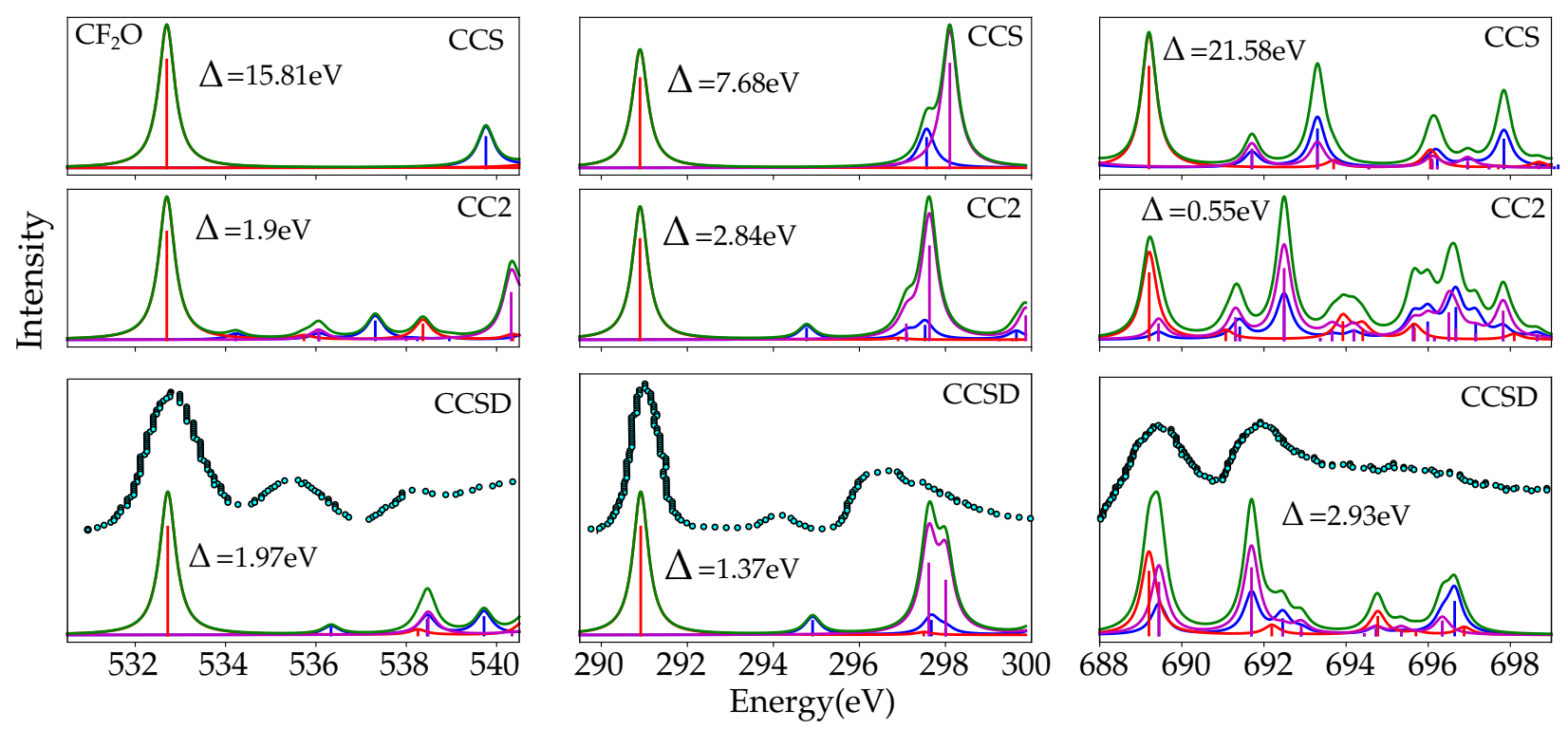

Figure 28: $\mathrm{CF}_{2} \mathrm{O}$ : eCVS-CCSD, eCVS-CC2 and eCVS-CCS carbon, oxygen and fluorine $\mathrm{K}$ edge spectra using the aug-cc-pCVTZ basis set. The symbol $\Delta$ indicates the shift applied to align the computed spectra with the first experimental peak. The experimental data are taken from ref. [262]. The total spectra are in green; blue lines and sticks refer to transitions of $A_{1}$ symmetry, red lines to those of $B_{1}$ symmetry and magenta lines to those of $B_{2}$ symmetry, for the molecule placed on the $y z$ plane and the $C_{2}$ axis along $z$.The spectral profiles shown have been generated by broadening the raw spectral data reported in Table 12 and 13 of the Appendix. 
pressed compared to eCVS-CCSD. The most striking differences between eCVS-CC 2 and eCVS-CCSD are observed for the fluorine K edges where, within the limits of the rather low experimental resolution, only eCVS-CCSD yields spectra in reasonably good agreement with the experimental ones.

Finally, as observed for formaldehyde, the core ionisation energies yielded by eCVS-CCSD are always overestimated by 1.5-3.1 eV, and the eCVS-CC2 ones are underestimated by $2-4 \mathrm{eV}$, depending on the edge. The deviations are not exactly the same as the systematic shifts $\Delta$, resulting in slightly higher (for eCVS-CCSD) or remarkably smaller (for eCVS-CC2) term values than the experimental counterparts, the difference increasing at higher energy K edges.

\subsection{VIBRONIC STRUCTURE OF THE BANDS}

As any other electronic spectroscopy, the simulation of XAS spectra directly comparable with experiments should account for the shape of the bands, which can arise from different mechanisms.

The finite lifetime of the excited states induces a natural broadening, especially relevant for high energy states, as it is the case in XAS spectroscopy. This contribution can be properly modelled by a Lorentzian convolution, as done in the spectra presented so far. In addition, the existence of quantum vibrational states of the chromophore leads to number of vibronic transitions that confers a well defined structure to the bands. Finally, interaction with the environment results in the so-called inhomogeneous broadening, which can be accounted for through a Gaussian convolution.

We will focus the analysis of the spectral shape on the $C 1 \mathrm{~s} \rightarrow \pi^{*}$ and $\mathrm{O} 1 \mathrm{~s} \rightarrow \pi^{*}$ bands in formaldehyde, which have been well characterized experimentally, including high resolution spectra [219]. The experimental results, shown in the bottom panels of Figures 20 and 23, indicate that such band is characterized by a of $\sim 1.5 \mathrm{eV}$. The shape seems structureless with this resolution, but in any case it is clearly different from the Lorentzian shape adopted to convolute the electronic sticks in the upper panels of Figure 23. As will shown below, such broadening mainly arises from the vibrational progressions.

Let us first address the simulation of the $\mathrm{C} 1 \mathrm{~s} \rightarrow \pi^{*}$ band. In this case, the high resolution experiments conducted by Remmers and coworkers,[219] not only confirm the broadening reported in Figures 20 and 23, but they also reveal a well resolved vibrational shape. In order to simulate the band, the vibronic spectrum have been computed at the harmonic approximation 
adopting the AH model, and computing the Hessian at ground and excited states minima with CCSD and equation of motion(EOM)-CCSD respectively. The symmetry and frequencies of the most important normal modes, together with a qualitative descriptions in terms of valence internal modes, are reported in Table 5.

All normal modes exhibit strong frequency changes in the different states. Moreover, while $v_{1}$ and $v_{4}$ correspond a unique internal coordinate, namely the symmetric $\mathrm{CH}$ stretching and the out-of-plane (oop) bending, $v_{2}$ and $v_{3}$ arise from the combination of two modes, the $\mathrm{CO}$ stretch and the in-plane $\mathrm{HCH}$ bending, with weights markedly dependent on the electronic state. In particular, while in the ground state the $\mathrm{CO}$ stretching mainly contributes to $v_{2}$, in the excited states, due to the decrease of its frequency it mixes remarkably with the $\mathrm{HCH}$ bending. This results in a strong Duschinsky mixing (A mode mixing by rotation of the vibrational normal coordinates in the electronic manyfolds [265]) of the corresponding normal modes. In the deuterated compound, the frequency of the $\mathrm{HCH}$ bending is remarkably lower, so that even in the excited states this mode mixes less effectively with the CO stretching.

In Figure 29, the spectra simulated at oK for both the deuterated and non-

Table 5: Formaldehyde: Most important vibrational modes and their frequencies in $\mathrm{cm}^{-1}$.

\begin{tabular}{c|c|c|ccc}
\hline Vib. & Sym. & Description $^{\mathrm{a}}$ & GS & $\mathrm{C} 1 \mathrm{~s} \rightarrow \pi^{*}$ & $\mathrm{O} 1 \mathrm{~s} \rightarrow \pi^{*}$ \\
\hline$v_{1}$ & $\mathrm{~A}_{1}$ & CH stretching & 2965 & 3582 & 3235 \\
$v_{2}$ & $\mathrm{~A}_{1}$ & CO stretch. +HCH bend. & 1823 & 1703 & 1564 \\
$v_{3}$ & $\mathrm{~A}_{1}$ & CO stretch. +HCH bend. & 1550 & 1529 & 1465 \\
$v_{4}$ & $\mathrm{~B}_{1}$ & oop bending & 1209 & 383 & $\mathrm{i}_{114}$ \\
\hline
\end{tabular}

${ }^{\mathrm{a}}$ Note that $v_{2}$ and $v_{3}$ undergo strong Duschinsky mixing

deuterated species are shown.

As observed in this figure, the simulations do provide similar vibrational features as compared with the experimental spectra of Remmers and coworkers, although with significant differences in the relative intensity along the progression. According to the simulations, the main vibrational progressions that characterize the spectrum arise from the $\mathrm{CH}$ stretching (mode $v_{1}$ ) and the stretching of the $\mathrm{C}=\mathrm{O}$ bond (contributing to both $v_{2}$ and $v_{3}$ ). In this sense, the differences between the simulated and observed progressions can be explained by the equilibrium values of such bond. At the (EOM-)CCSD 
level, the $\mathrm{C}=\mathrm{O}$ equilibrium bond length at the ground and excited states are 1.202 and $1.266 \AA$, respectively. These values should be compared with those estimated by [219], [219] which are 1.207 and $1.316 \AA$ for ground and C $1 \mathrm{~s} \rightarrow \pi^{*}$ states, respectively.

It is thus evident that while the simulated and experimentally derived values at the ground state are in reasonable agreement, the elongation in the excited state is considerably underestimated in the EOM-CCSD calculation, which is consistent with the less pronounced progression observed in the simulated spectrum.

This error can be attributed to the limitations of EOM-CCSD to properly reproduce the excited state PES. This indicates that the inclusion of triples and quadruples corrections would be of interest to further improve the results. This, however, goes beyond the present work that focuses on the assessment of the performance of practical CCSD approach.

Trofimov et al. [266] explored the effect on the spectral shape of a coupling, promoted by the $v_{4}$ out-of-plane bending motion, between $\mathrm{C} 1 \mathrm{~s} \rightarrow \pi^{*}$ and $\mathrm{C} 1 \mathrm{~s} \rightarrow 3 \mathrm{~s}$ state, lying $\sim 4.6 \mathrm{eV}$ above the lower one. They adopted a linear vibronic coupling model (LVC) parametrized with ADC(2) and MRCI data, while the inter-state coupling was fitted to reproduce a double-well profile of C $1 \mathrm{~s} \rightarrow \pi^{*}$ along $v_{4}$ mode, predicted with ROHF calculations [267]. They found that interstate coupling only slightly affects the spectral shapes, introducing a significant progression along the coupling mode $v_{4}$.

According to the present calculations, there is a single minimum along $v_{4}$ that preserves the $C_{2 v}$ symmetry. Notwithstanding this, it can be also predicted a progression with even quantum numbers along the $B_{1}$ mode $v_{4}$ due to the strong decrease of its frequency (see Figure 29). The overall shape of [263]'s spectrum is therefore very similar even if the coupling is neglected, as it is done in this case. As far as the relative intensity of the main bands is concerned, the predictions of [263] [266] are in better agreement with the experiment with respect to the present results. This is due to the larger displacement of the equilibrium distance of the $\mathrm{CO}$ bond predicted at ADC(2) level of theory (1.28o $\AA$ in the C $1 \mathrm{~s} \rightarrow \pi^{*}$ state) [266]. On the other side, the displacement of the $\mathrm{CH}$ stretching is pretty similar: in the excited state it is $1.02 \AA$ according to the present calculations and $1.03 \AA$ in Trofimov's model. Our calculations accurately reproduce the spacings of the vibronic bands, thanks to the fact that the $\mathrm{AH}$ model explicitly accounts for the large frequency changes undergone by $\mathrm{CO}$ and $\mathrm{CH}$ stretching (see Table 5) and for the Duschinsky mixings. A calculation with AS model [244] that, analogously to the LVC model adopted by Trofimov et al., assumes 
that the excited-state normal modes and frequencies are equal to those of the ground state, leads to clearly less satisfactory results (see Figure 31 of the Appendix).

The agreement with the spectrum of the deuterated species is also satisfactory. In particular, the enhancement of the $I^{1}$ band has been reproduced since, due to the larger mass of the $\mathrm{D}$ isotope, the dimensionless displacement increases from 1.10 ( $\mathrm{CH}$ stretch) to 1.38 (CD stretch), and, at the same time, its frequency reduces from $3582 \mathrm{~cm}^{-1}$ (Table 5) to $2593 \mathrm{~cm}^{-1}$. On the contrary, the $\mathrm{C}=\mathrm{O}$ stretching mode is practically unaltered, but, due to the decoupling with the DCD bending, the Duschinsky mixing is very limited and a single $2^{1}$ band substitutes the $2^{1}, 3^{1}$ pair in the non-deuterated species.

The attention can now be turned to the $\mathrm{O} 1 \mathrm{~s} \rightarrow \pi^{*}$ band.

In this case, the excited state geometry has a lower symmetry $\left(C_{s}\right)$ as compared to the ground state minimum $\left(\mathrm{C}_{2 v}\right)$. Concretely, whilst the structure is planar in the ground state, it is partially pyramidalised along the $v_{4}$ oop bending mode in the excited state, and the potential energy profile along the improper dihedral that breaks the planarity is characterized by a doublewell. This scenario prevents the adoption of the harmonic approximation, at least to describe the PES along such degree of freedom.

Trofimov et al. [263] attributed this feature to a coupling with a $\mathrm{O} 1 \mathrm{~s} \rightarrow 3 \mathrm{~s}$ state and computed the spectra with a LVC vibronic model. In this case the effect of vibronic coupling is larger than for $\mathrm{C} 1 \mathrm{~s} \rightarrow \pi^{*}$ but it remains moderate, as it mainly introduces a broadening, smearing out the main vibronic bands. The parametrization of a full dimensionality LVC model on the grounds of EOM-CCSD data might be done generalizing the strategies have been adopted in combination with TD-DFT for some nucleobases, [268270] but this lies beyond the scope of this work. Therefore, a simpler strategy has been adopted and removed the mode $v_{4}$ from the harmonic calculations. This implicitly constrains the equilibrium geometry to $\mathrm{C}_{2 v}$ symmetry, analogously to what is obtained with a LVC model neglecting the inter-state coupling.

The computed spectrum is shown in Figure 30, where vibronic progressions along modes $v_{2}$ and $v_{3}$, both connected to the CO stretching, are clearly visible. Figure $30 \mathrm{~b}$ compares the computed spectrum (red shifted by $1.72 \mathrm{eV}$ ) to the experimental one taken from ref. [263], showing that the predicted shape is remarkably narrower than the experimental one (and the LVC one computed in ref. [263]). Similar to what happens for the C $1 \mathrm{~s} \rightarrow \pi^{*}$ spectrum, the main difference of the present calculations with respect to those 


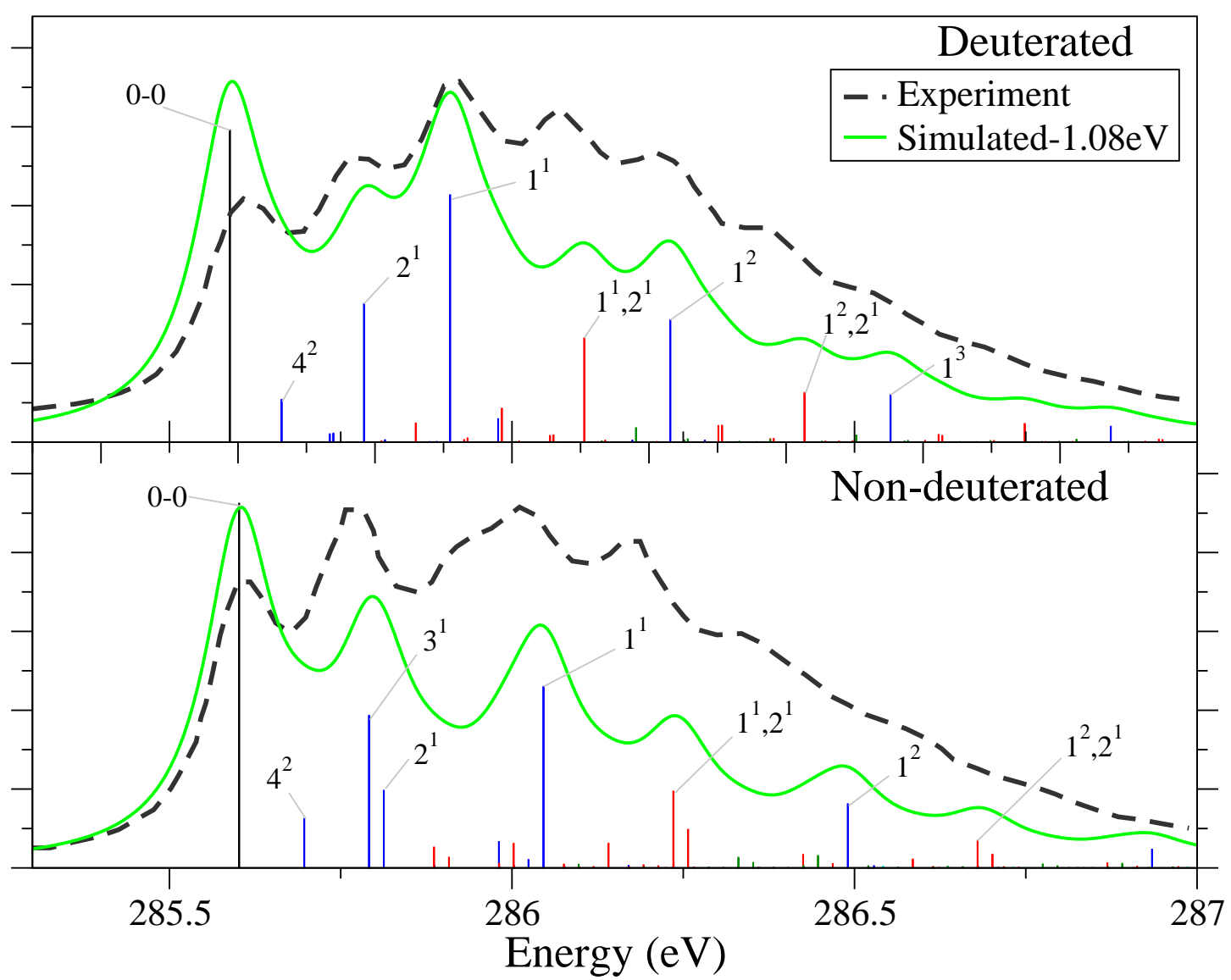

Figure 29: Formaldehyde: Simulation of the $\mathrm{C} 1 \mathrm{~s} \rightarrow \pi^{*}$ band at oK for deuterated and non-deuterated species, computed on the grounds of EOM-CCSD PES, adopting a Lorentzian broadening of $=0.12 \mathrm{eV}$. Note that the simulated spectra are red-shifted by $1.08 \mathrm{eV}$ so as to match the position of the experimental bands, taken from ref. [219]. Vertical lines show the vibronic transitions from the vibrational ground state in the initial electronic state. The labels correspond to the vibrational state in the final electronic states, represented as $X^{y}$, where $X$ is the normal mode index and $y$ the number of quanta. 
of [263] is connected to the underestimation of the elongation of the $C=O$ bond-length - that is, $1.30 \AA$ in the present calculations versus $\sim 1.34 \AA$ in their LVC model. This is proven in Figure $30 \mathrm{~b}$ recomputing the spectrum by artificially increasing the CO bond length of the excited state up to $\sim 1.34 \AA$ and showing that the spectral shape obtained in this case is in fair agreement with experiment.

The LVC assumption of equal frequencies in the ground and excited states makes the bands much more spaced and therefore resolved, giving overall a not satisfactory agreement with experiment [263] . In ref. [263],phenomeno-
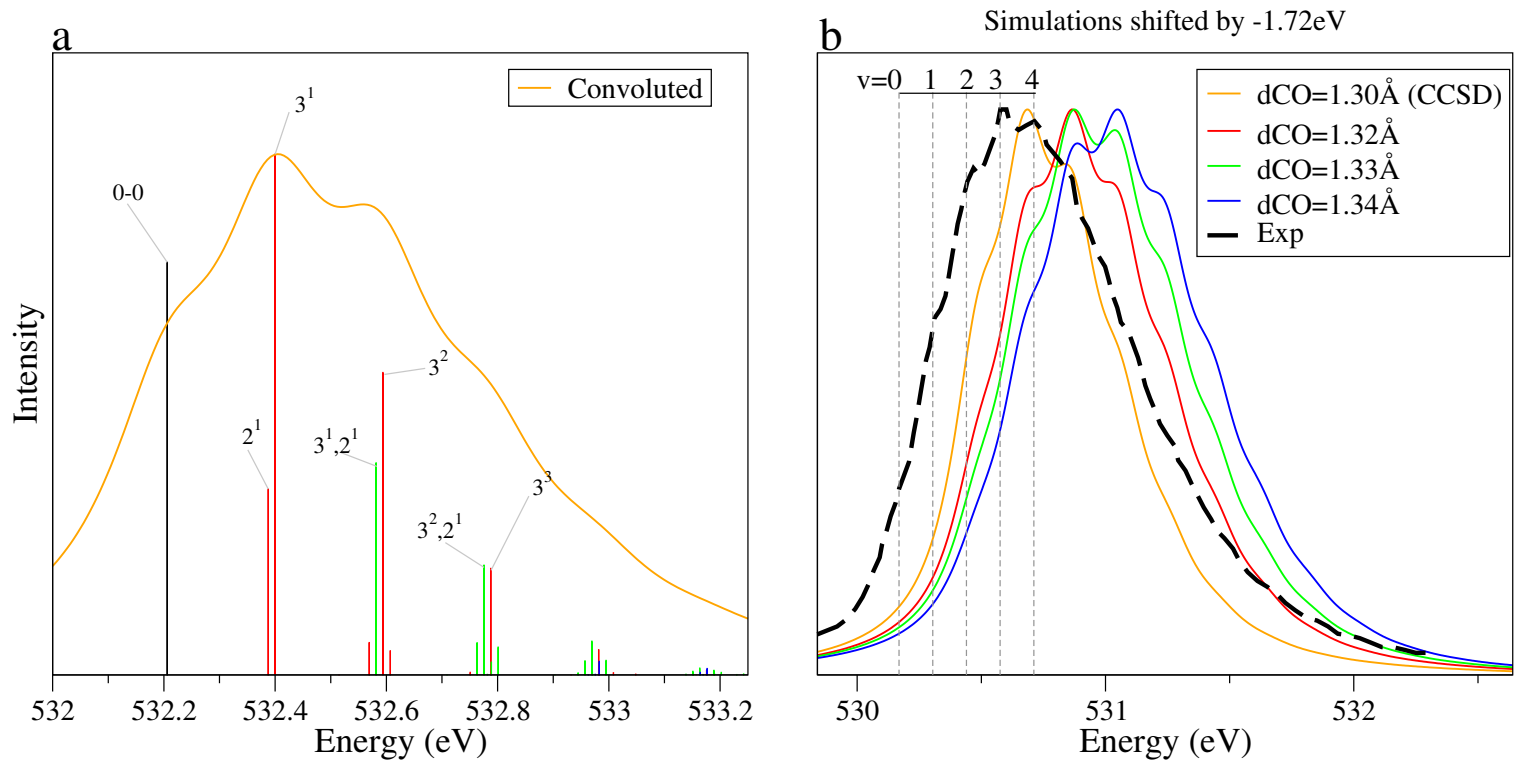

Figure 30: Formaldehyde. Panel a: Simulation of the O1s $\rightarrow \pi^{*}$ band at oK, on the grounds of EOM-CCSD PES, adopting a Lorentzian broadening of $\mathrm{HWHM}=0.13 \mathrm{eV}$. The spectra are computed at the $\mathrm{C}_{2 v}$ stationary point in the excited state, removing the out-of-plane bending which presents a double-well type anharmonic profile in the excited state. Vertical lines show the vibronic transitions, similar to what presented in Figure 29. Modes 2 and 3 correspond to a combination of $\mathrm{C}=\mathrm{O}$ stretching and $\mathrm{H}-\mathrm{C}-\mathrm{H}$ bending. Panel b: Comparison with experiment. Different computed spectra have been obtained by artificially increasing the $\mathrm{CO}$ bond length in the excited state up to $1.34 \AA$. Computed spectra have been all red-shifted by $1.72 \mathrm{eV}$. This value was chosen to better superimpose the spectrum directly obtained by CCSD data (i.e. with $\mathrm{dCO}=1.30 \AA$ ) to the experiment. Vertical dashed lines show the main progression as assigned from the experiment [263].

logically corrected for this error with a modified LVC model, where excited state frequencies were scaled. For example, while the CO stretch frequency 
is $1751 \mathrm{~cm}^{-1}$ in the ground state, it matches the experimental value, 1079 $\mathrm{cm}^{-1}$, in the $\mathrm{O} 1 \mathrm{~s} \rightarrow \pi^{*}$ state. In principle the AH model accounts for frequency changes and, in fact, it is predicted a remarkable decrease of frequency of 260 and $85 \mathrm{~cm}^{-1}$ for the two modes $v_{2}$ and $v_{3}$ with contributions from the $\mathrm{CO}$ stretch. This goes in the direction of the experimental observation, but the frequency change is underestimated. As a consequence the spacing of the computed vibronic bands is still too large with respect to experiment (check the vertical lines plotted in Figure 3ob). This finding is in line with the underestimation of the elongation of the $\mathrm{CO}$ bond length. In fact it is reasonable that the longer the bond becomes the lower is its vibrational frequency. It is also possible that remarkable anharmonic effects play a role in the excited state.

Going back to the broadening induced by progressions along mode $v_{4}$, neglected in the present calculations, in the Figure 32 of the Appendix it is also reported, a one-dimensional scan of the $\mathrm{O} 1 \mathrm{~s} \rightarrow \pi^{*}$ along the $v_{4}$ mode in internal coordinates which shows that, at the adopted level of theory, the $\mathrm{C}_{2 v}$ barrier is quite low $\left(0.3-0.5 \mathrm{~cm}^{-1}\right)$, not supporting any bound state. Moreover, the data in Table 16(see Appendix) indicate that, apart from the value of the oop angle, all other geometrical parameters are practically identical in the $\mathrm{C}_{2 v}$ transition-state and in the true $\mathrm{C}_{s}$ minimum. In this situation the energy profile might be approximately substituted by a harmonic curve. We therefore pragmatically checked the possible effect of a frequency change on $v_{4}$ mode by replacing the double-well profile with a harmonic one in the vibronic calculations, attributing to $v_{4}$ different harmonic frequencies, from $\sim 100$ to $\sim 2000 \mathrm{~cm}^{-1}$ (see Figure 33 of the Appendix). The predicted spectrum changes only slightly, confirming that for this transition, a full LVC treatment including inter-state couplings is necessary to capture the effect $v_{4}$ of on the spectrum.

\section{$3 \cdot 5$ CONCLUSIONS}

The capability of the core-valence-separated CC methods eCVS-CCS, eCVSCC2 and eCVS-CCSD to yield reliable core spectra has been tested at the carbon, oxygen and fluorine $\mathrm{K}$ edges of formaldehyde, formic acid, formyl fluoride and carbonyl fluoride, with some emphasis on the first is $\rightarrow \pi^{*}$ transition as well as the region of Rydberg transitions (in the case of formaldehyde).

Different basis sets of double, triple and quadruple- $\zeta$ quality have been tested with augmenting diffuse functions, core polarization functions and 
Rydberg functions. While eCVS-CCS, not surprisingly, always fails in describing the core excitations at all $\mathrm{K}$ edges, the eCVS-CC2 approach yields XAS spectra quite similar to eCVS-CCSD at the carbon K edge, apart from a different systematic shift compared to experiment. At the oxygen $\mathrm{K}$ edge, the eCVS-CC2 spectra are typically "compressed" compared to the eCVSCCSD ones, i.e., the higher energy excitations fall too close to the dominant is $\rightarrow \pi^{*}$ band. At the fluorine K edge, eCVS-CC 2 and eCVS-CCSD spectra are very different, and using eCVS-CC2 is not advisable.

The eCVS-CCSD scheme is found to yield spectra in satisfactory agreement with experiment at all edges, once a systematic realignment has been applied and an appropriate basis set is chosen.

The eCVS-CCSD IEs are also found in satisfactory agreement with experiment, though at instances slightly misaligned with respect to the systematic shift of the absorption spectrum, yielding term values which are usually slightly overestimated. The eCVS-CC2 IEs are always too low, yielding too low term values. Also the $\mathrm{C} 1 \mathrm{~s} \rightarrow \pi^{*}$ and $\mathrm{O} 1 \mathrm{~s} \rightarrow \pi^{*}$ vibrationally resolved spectra of formaldehyde have been computed, with the AH harmonic model, on the grounds of (equation of motion)EOM-CCSD quadratic expansions of the ground and excited states. The overall agreement with experiment is reasonable and qualitatively reproduces the observed isotopic effects. Accounting for the differences of the energy Hessians of the initial and final states of the transitions, introduces frequency changes and Duschinsky mixings that remarkably improve the spacings of the main vibronic bands, although for the $\mathrm{O} 1 \mathrm{~s} \rightarrow 3 \mathrm{~s}$ state there are still too large probably due to important anharmonic effects. Although the inter-state electronic couplings are neglected, which according to Trofimov et al. [263, 266, 267] have some moderate effects on the spectral shapes, most of the inaccuracies of our computed spectra can be traced back to the underestimation of the elongation of the $\mathrm{CO}$ bond in the excited states. This indicates that for accurate vibronic lineshapes of these core-excitations inclusion of the effect of triple excitations scheme may be necessary. 


\section{APPENDIX CHAPTER III}

Table 6: Formaldehyde: eCVS-CCSD oxygen core-excitation energies (eV) and oscillator strengths $f$ in different correlation consistent basis sets supplemented with (3s3p3d) Rydberg functions

\begin{tabular}{|c|c|c|c|c|c|c|}
\hline \multirow[t]{3}{*}{ Symm. } & \multirow{2}{*}{\multicolumn{2}{|c|}{$\begin{array}{c}\text { Energy } \quad f \\
\text { aug-cc-pCVDZ+Ryd }\end{array}$}} & \multirow{2}{*}{\multicolumn{2}{|c|}{$\begin{array}{cc}\text { Energy } & f \\
\text { aug-cc-pCVTZ+Ryd }\end{array}$}} & \multirow{2}{*}{\multicolumn{2}{|c|}{$\begin{array}{l}\text { Energy } \quad f \\
\text { aug-cc-pCVQZ+Ryd }\end{array}$}} \\
\hline & & & & & & \\
\hline & & & Oxygen & K-edge & & \\
\hline \multirow{14}{*}{$\mathrm{A}_{1}$} & $539 \cdot 78885$ & 0.0004846 & 537.85000 & 0.0004889 & $537 \cdot 77246$ & 0.0004818 \\
\hline & 540.48393 & 0.0010109 & 538.54770 & 0.0009664 & 538.46686 & 0.0010093 \\
\hline & 540.48393 & 0.0005714 & $539 \cdot 70494$ & 0.0004462 & 539.61665 & 0.0002978 \\
\hline & 541.66363 & 0.0007018 & $539 \cdot 79343$ & 0.0008833 & 539.69237 & 0.0010840 \\
\hline & 541.77237 & 0.0004769 & 539.96586 & 0.0005671 & 539.88850 & 0.0005848 \\
\hline & 542.05616 & 0.0002338 & 540.12126 & 0.0002083 & 540.04564 & 0.0002194 \\
\hline & 542.43577 & 0.0002577 & 540.48577 & 0.0001396 & 540.40094 & 0.0000542 \\
\hline & 542.48744 & 0.0004902 & 540.52640 & 0.0005904 & 540.43895 & o.0006877 \\
\hline & 542.56060 & 0.0003122 & 540.61669 & 0.0003609 & 540.53960 & 0.0003803 \\
\hline & 542.63597 & 0.0000433 & 540.70035 & 0.0000553 & 540.62499 & 0.0000634 \\
\hline & 542.84149 & 0.0000482 & 540.90255 & 0.0000170 & 540.82765 & 0.0000204 \\
\hline & 542.98268 & 0.0008530 & 541.01203 & 0.0003641 & 540.92358 & 0.0002993 \\
\hline & 543.06691 & 0.0010128 & 541.10496 & 0.0014818 & 541.02286 & 0.0015575 \\
\hline & $543 \cdot 32126$ & 0.0002949 & 541.37434 & 0.0001722 & 541.28784 & 0.0001394 \\
\hline \multirow{8}{*}{$\mathrm{B}_{1}$} & 534.47942 & 0.0450774 & 532.52234 & o.0459095 & 532.38615 & 0.0461831 \\
\hline & 540.90973 & 0.0001107 & 538.95845 & 0.0001288 & 538.87814 & 0.0001349 \\
\hline & 541.90138 & 0.000708o & 539.95757 & o.0007574 & 539.87716 & 0.0007866 \\
\hline & 542.17684 & 0.0000442 & 540.23419 & 0.0000476 & 540.15756 & 0.0000486 \\
\hline & 542.57315 & 0.0004232 & 540.63038 & 0.0004597 & 540.55091 & 0.0004734 \\
\hline & 542.69044 & 0.0000187 & 540.75332 & 0.0000214 & 540.67962 & 0.0000208 \\
\hline & 543.16330 & 0.0450774 & 541.22039 & o.0008989 & 541.14145 & 0.0008953 \\
\hline & 543.63052 & 0.0001107 & 541.60775 & 0.0000620 & 541.48377 & 0.0000510 \\
\hline \multirow{7}{*}{$\mathrm{B}_{2}$} & 540.74849 & 0.0000601 & 538.79814 & 0.0000669 & 538.71968 & 0.0000675 \\
\hline & 541.80833 & 0.0024765 & 539.86227 & 0.0025457 & $539 \cdot 77951$ & 0.0026824 \\
\hline & 542.12381 & 0.0000286 & 540.18306 & 0.0000293 & 540.10780 & 0.0000292 \\
\hline & 542.51562 & 0.0013708 & 540.57297 & 0.0014087 & 540.49286 & 0.0014695 \\
\hline & 542.66183 & 0.0000309 & 540.72367 & 0.0000224 & 540.64883 & 0.0000200 \\
\hline & 543.06553 & 0.0022366 & 541.12097 & 0.0023012 & 541.04037 & 0.0023319 \\
\hline & $543 \cdot 35943$ & 0.0090399 & 542.69119 & 0.0090726 & 541.27970 & 0.0068934 \\
\hline
\end{tabular}


Table 7: Formaldehyde: eCVS-CCSD carbon core-excitation energies (eV) and oscillator strengths $f$ in different correlation consistent basis sets supplemented with (3s3p3d) Rydberg functions

\begin{tabular}{|c|c|c|c|c|c|c|}
\hline \multirow[t]{2}{*}{ Symm. } & \multicolumn{2}{|c|}{ Energy $\quad f$} & \multicolumn{2}{|l|}{ Energy } & \multicolumn{2}{|l|}{ Energy } \\
\hline & \multicolumn{2}{|c|}{ aug-cc-pCVDZ+Ryd } & \multicolumn{2}{|c|}{ aug-cc-pCVTZ+Ryd } & \multicolumn{2}{|c|}{ aug-cc-pCVQZ+Ryd } \\
\hline \multirow{19}{*}{$\mathrm{A}_{1}$} & \multicolumn{6}{|c|}{ Carbon K-edge } \\
\hline & 292.77150 & 0.0084936 & 291.73933 & 0.0087741 & 291.70546 & 0.0089769 \\
\hline & 294.02327 & 0.0001210 & 292.97116 & 0.0001355 & 292.94415 & 0.0001498 \\
\hline & 294.99137 & 0.0022979 & 293.89225 & 0.0023399 & 293.84167 & 0.0022952 \\
\hline & 295.40949 & 0.0001463 & $294 \cdot 36064$ & 0.0002146 & 294.32960 & 0.0002590 \\
\hline & 295.46132 & 0.0001394 & 294.42255 & 0.0001544 & $294 \cdot 39754$ & 0.0001687 \\
\hline & 295.62989 & 0.0001540 & 294.60478 & 0.0001580 & 294.58631 & 0.0001710 \\
\hline & 295.90913 & 0.0008713 & 294.85382 & 0.0008016 & 294.82535 & 0.0007668 \\
\hline & 296.10621 & 0.0000321 & 295.06801 & 0.0000782 & 295.04310 & 0.0001031 \\
\hline & 296.12877 & 0.0000311 & 295.09847 & 0.0000447 & 295.07714 & 0.0000470 \\
\hline & 296.22621 & 0.0000890 & 295.20351 & 0.0000901 & 295.18522 & 0.0000950 \\
\hline & 296.35476 & 0.0007481 & $295 \cdot 32593$ & 0.0006637 & $295 \cdot 30733$ & 0.0006798 \\
\hline & 296.54712 & 0.0000079 & 295.48307 & 0.0000001 & 295.45633 & 0.0000004 \\
\hline & 296.66051 & 0.0000709 & 295.62165 & 0.0000379 & $295 \cdot 59782$ & 0.0000321 \\
\hline & 296.91096 & 0.0000918 & 295.87289 & 0.0000591 & 295.84198 & 0.0000397 \\
\hline & $297 \cdot 42066$ & 0.0037294 & 296.21453 & 0.0028474 & 296.14422 & 0.0026116 \\
\hline & & & 296.91342 & 0.0003544 & 296.75803 & 0.0002527 \\
\hline & & & $297 \cdot 50282$ & 0.0000310 & 297.21275 & 0.0000063 \\
\hline & & & & & 297.67580 & 0.0033925 \\
\hline \multirow{9}{*}{$\mathrm{B}_{1}$} & 288.21431 & 0.0582141 & 287.01086 & 0.0613778 & 286.90420 & 0.0624286 \\
\hline & $294 \cdot 31367$ & 0.0066376 & 293.27771 & 0.0069033 & 293.25083 & 0.0069196 \\
\hline & $295 \cdot 52454$ & 0.0000683 & 294.49498 & 0.0000905 & 294.46949 & 0.0001078 \\
\hline & 295.70464 & 0.0021269 & 294.67824 & 0.0021814 & 294.65805 & 0.0021627 \\
\hline & 296.18315 & 0.0000482 & 295.15535 & 0.0000732 & 295.13168 & 0.0000865 \\
\hline & 296.24441 & 0.0010224 & 295.22284 & 0.0012007 & 295.20549 & 0.0011698 \\
\hline & 296.78252 & 0.0002852 & 295.74891 & 0.0003197 & $295 \cdot 72082$ & 0.0003322 \\
\hline & 297.04305 & 0.0050630 & 295.97156 & 0.0038880 & 295.92235 & 0.0034590 \\
\hline & & & & & 297.72331 & 0.0001569 \\
\hline \multirow{11}{*}{$\mathrm{B}_{2}$} & 293.76042 & 0.0227911 & 292.71620 & 0.0242282 & 292.67279 & 0.0251353 \\
\hline & $295 \cdot 41986$ & 0.0037092 & $294 \cdot 38051$ & 0.0041841 & $294 \cdot 34989$ & 0.0043190 \\
\hline & $295 \cdot 56145$ & 0.0034776 & 294.53610 & 0.0035925 & $294 \cdot 51428$ & 0.0036537 \\
\hline & 296.10577 & 0.0022820 & 295.07344 & 0.0024529 & 295.04802 & 0.0024327 \\
\hline & 296.18730 & 0.0010990 & 295.16360 & 0.0011782 & 295.14375 & 0.0012328 \\
\hline & 296.60108 & 0.0041133 & 295.56460 & 0.0040992 & 295.53670 & 0.0040194 \\
\hline & 296.82701 & 0.0017933 & $295 \cdot 78433$ & 0.0015947 & $295 \cdot 75436$ & 0.0014801 \\
\hline & $297 \cdot 74724$ & 0.0002083 & 296.93597 & 0.0143566 & 296.76582 & 0.0124277 \\
\hline & & & $297 \cdot 37434$ & 0.0005656 & 297.11395 & 0.0003308 \\
\hline & & & $297 \cdot 55816$ & 0.0005884 & $297 \cdot 57444$ & 0.0005168 \\
\hline & & & & & 297.66745 & 0.0000269 \\
\hline
\end{tabular}


Table 8: Formaldehyde: eCVS-CC2 oxygen and carbon core-excitation energies (eV) and oscillator strengths $f$ in the aug-cc-pCVTZ basis set supplemented with Rydberg functions

\begin{tabular}{|c|c|c|c|c|}
\hline \multirow{3}{*}{ Symm. } & \multicolumn{2}{|c|}{ O K-edge } & \multicolumn{2}{|c|}{ C K-edge } \\
\hline & Energy $(\mathrm{eV})$ & $f$ & Energy $(\mathrm{eV})$ & $f$ \\
\hline & \multicolumn{4}{|c|}{$\mathrm{CC}_{2}$} \\
\hline \multirow{17}{*}{$\mathrm{A}_{1}$} & 535.02873 & 0.0005221 & 292.85609 & 0.0071903 \\
\hline & 535.83400 & 0.0000667 & 293.87971 & 0.0000498 \\
\hline & 536.47966 & 0.0000265 & 294.62746 & 0.0021415 \\
\hline & 536.61152 & 0.0000000 & 294.86386 & 0.0000334 \\
\hline & 536.65520 & 0.0002718 & 294.91252 & 0.0000683 \\
\hline & 536.75204 & 0.0000129 & 295.02242 & 0.0000497 \\
\hline & 536.96487 & 0.0000071 & 295.24744 & 0.0009027 \\
\hline & 537.08040 & 0.0000006 & 295.37929 & 0.0000029 \\
\hline & $537 \cdot 10767$ & 0.0002913 & $295 \cdot 40871$ & 0.0000408 \\
\hline & 537.19607 & 0.0000005 & $295 \cdot 49756$ & 0.0000070 \\
\hline & $537 \cdot 36907$ & 0.0000102 & 295.62976 & 0.0010843 \\
\hline & 537.64318 & 0.0000068 & 295.88434 & 0.0000910 \\
\hline & $537 \cdot 78172$ & 0.0008855 & 296.02217 & 0.0001132 \\
\hline & 538.14518 & 0.0001566 & 296.31935 & 0.0000095 \\
\hline & & & 296.89955 & 0.0050224 \\
\hline & & & 297.68034 & 0.0000700 \\
\hline & & & 298.26063 & 0.0003951 \\
\hline \multirow{9}{*}{$\mathrm{B}_{1}$} & 532.53777 & 0.0402327 & 288.52602 & 0.0673590 \\
\hline & 535.98693 & 0.0000406 & 294.04041 & 0.0048693 \\
\hline & 536.67589 & 0.0003517 & 294.95511 & 0.0000715 \\
\hline & 536.79768 & 0.0000093 & 295.06089 & 0.0015309 \\
\hline & $537 \cdot 15425$ & 0.0003782 & $295 \cdot 46749$ & 0.0001633 \\
\hline & 537.22968 & 0.0000027 & $295 \cdot 50890$ & 0.0011809 \\
\hline & 537.87139 & 0.0011250 & 296.14221 & 0.0004014 \\
\hline & 538.43248 & 0.0000037 & 296.47658 & 0.0050810 \\
\hline & & & 298.94865 & 0.0002827 \\
\hline \multirow{9}{*}{$\mathrm{B}_{2}$} & 535.82141 & 0.0000027 & 293.70487 & 0.0168184 \\
\hline & 536.66839 & 0.0003574 & 294.90079 & 0.0026081 \\
\hline & 536.74510 & 0.0000005 & 294.97198 & 0.0028936 \\
\hline & 537.13930 & 0.0003808 & 295.40330 & 0.0027295 \\
\hline & $537 \cdot 17774$ & 0.0000135 & $295 \cdot 46283$ & 0.0008391 \\
\hline & 537.84331 & 0.0012872 & 295.98310 & 0.0059466 \\
\hline & 537.99267 & 0.000002 & 296.17061 & 0.0023482 \\
\hline & & & 297.69462 & 0.0205921 \\
\hline & & & 298.15698 & 0.0018760 \\
\hline
\end{tabular}


Table 9: Formaldehyde: eCVS-CCS oxygen and carbon core-excitation energies (eV) and oscillator strengths $f$ in the aug-cc-pCVTZ basis set supplemented with Rydberg functions

\begin{tabular}{|c|c|c|c|c|}
\hline \multirow{3}{*}{ Symm. } & \multicolumn{2}{|c|}{ O K-edge } & \multicolumn{2}{|c|}{ C K-edge } \\
\hline & Energy $(\mathrm{eV})$ & $f$ & Energy $(\mathrm{eV})$ & $f$ \\
\hline & \multicolumn{4}{|c|}{ CCS } \\
\hline \multirow{14}{*}{$\mathrm{A}_{1}$} & $555 \cdot 78069$ & 0.0001735 & 302.35479 & 0.0311222 \\
\hline & 556.51554 & 0.0324728 & 305.22567 & 0.0036570 \\
\hline & 557.67707 & 0.0116590 & 305.98473 & 0.0029548 \\
\hline & 558.31862 & 0.0017732 & 306.90455 & 0.0002308 \\
\hline & 558.39625 & 0.0006101 & 307.06415 & 0.0011212 \\
\hline & 558.56919 & 0.0012852 & 307.25352 & 0.0012207 \\
\hline & 558.81125 & 0.0028543 & $307 \cdot 37603$ & 0.0007665 \\
\hline & 559.04457 & 0.0005898 & 307.68271 & 0.0001031 \\
\hline & 559.08841 & 0.0003333 & $307 \cdot 74671$ & 0.0006441 \\
\hline & 559.17802 & 0.0003851 & 307.87131 & 0.0010469 \\
\hline & $559 \cdot 32831$ & 0.0024440 & 307.92236 & 0.0005622 \\
\hline & 559.42769 & 0.0005115 & 308.09276 & 0.0000638 \\
\hline & 559.58829 & 0.0001870 & 308.23708 & 0.0004612 \\
\hline & $559 \cdot 76712$ & 0.0001666 & 308.49167 & 0.0028123 \\
\hline \multirow{8}{*}{$\mathrm{B}_{1}$} & 545.94644 & 0.0790750 & 294.28606 & 0.1121557 \\
\hline & $557 \cdot 41551$ & 0.0004265 & 305.44159 & 0.0081316 \\
\hline & 558.43586 & 0.0008663 & 307.20643 & 0.0013270 \\
\hline & 558.72844 & 0.0001239 & 307.21499 & 0.0009624 \\
\hline & 559.11190 & 0.0005291 & 307.82701 & 0.0011025 \\
\hline & 559.26116 & 0.0000353 & 307.85470 & 0.0000000 \\
\hline & 559.66828 & 0.0009816 & 308.40733 & 0.0018502 \\
\hline & & & 308.49261 & 0.0008178 \\
\hline \multirow{7}{*}{$\mathrm{B}_{2}$} & 557.13556 & 0.0110047 & 302.49133 & 0.0922398 \\
\hline & 557.86638 & 0.0135577 & 306.72154 & 0.0061949 \\
\hline & 558.68004 & 0.0005234 & 307.18340 & 0.0000967 \\
\hline & 558.89857 & 0.0033531 & 307.62001 & 0.0020590 \\
\hline & 559.23403 & 0.0001138 & 307.83174 & 0.0000718 \\
\hline & 559.41334 & 0.0029258 & 308.09048 & 0.0017635 \\
\hline & 559.92357 & 0.0000002 & 308.44481 & 0.0003277 \\
\hline
\end{tabular}


Table 10: CHFO: eCVS-CCSD, eCVS-CC 2 and eCVS-CCS oxygen core-excitation energies (eV) and oscillator strengths in the aug-cc-pCVTZ basis set

\begin{tabular}{|c|c|c|c|c|c|c|}
\hline & \multicolumn{2}{|c|}{ eCVS-CCSD } & \multicolumn{2}{|c|}{ eCVS-CC2 } & \multicolumn{2}{|c|}{ eCVS-CCS } \\
\hline \multicolumn{7}{|c|}{ Oxygen K-edge } \\
\hline \multirow{15}{*}{$A^{\prime}$} & 538.52650 & 0.0007129 & 535.86168 & 0.0004991 & 556.16517 & 0.0055993 \\
\hline & 539.16558 & 0.0022780 & 536.62495 & 0.0012301 & 556.74043 & 0.0304170 \\
\hline & 539.62247 & 0.0066394 & 537.06353 & 0.0024191 & 557.26000 & 0.0336164 \\
\hline & 540.99934 & 0.0057395 & 538.38412 & 0.0003075 & 558.17388 & 0.0169250 \\
\hline & 541.52739 & 0.0034845 & 538.77129 & 0.0035268 & $559 \cdot 78856$ & 0.0049805 \\
\hline & 541.77626 & 0.0008921 & $539 \cdot 30878$ & 0.0012317 & 560.05149 & 0.0007838 \\
\hline & 542.24787 & $0.0055^{141}$ & 539.67948 & 0.0027253 & 560.14322 & 0.0082407 \\
\hline & 542.87934 & 0.0053812 & 540.66459 & 0.0075874 & 560.82511 & 0.0023399 \\
\hline & $543 \cdot 51093$ & 0.0031379 & 541.03950 & 0.0018885 & 561.69478 & 0.0054101 \\
\hline & $544 \cdot 72037$ & 0.0001320 & 542.00714 & 0.0000081 & 562.30699 & 0.0147092 \\
\hline & 545.06407 & 0.0051406 & & & & \\
\hline & 545.17104 & 0.0019215 & & & & \\
\hline & 546.34016 & 0.0060640 & & & & \\
\hline & 547.12313 & 0.0020178 & & & & \\
\hline & $547 \cdot 41781$ & 0.0021091 & & & & \\
\hline \multirow{7}{*}{$\mathrm{A}^{\prime \prime}$} & 533.65846 & 0.0424591 & 533.53995 & 0.0366370 & $547 \cdot 35169$ & 0.0743963 \\
\hline & $539 \cdot 75619$ & 0.0004117 & 537.01297 & 0.0000101 & 558.10673 & 0.0009565 \\
\hline & 542.08096 & 0.0056348 & $539 \cdot 74035$ & 0.0064621 & 559.98328 & 0.0045991 \\
\hline & 543.44189 & 0.0005308 & 540.77400 & 0.0011957 & 562.08505 & 0.0004011 \\
\hline & 544.49380 & 0.0000430 & 541.65886 & 0.0000021 & & \\
\hline & $546.4345^{1}$ & 0.0000059 & & & & \\
\hline & $547 \cdot 17102$ & 0.0042027 & & & & \\
\hline
\end{tabular}


Table 11: CHFO: eCVS-CCSD, eCVS-CC 2 and eCVS-CCS carbon and fluorine coreexcitation energies (eV) and oscillator strengths in the aug-cc-pCVTZ basis set

\begin{tabular}{|c|c|c|c|c|c|c|}
\hline & \multicolumn{2}{|c|}{ eCVS-CCSD } & \multicolumn{2}{|c|}{ eCVS-CC2 } & \multicolumn{2}{|c|}{ eCVS-CCS } \\
\hline \multicolumn{7}{|c|}{ Carbon K-edge } \\
\hline \multirow{13}{*}{$A^{\prime}$} & 294.51050 & 0.0177774 & 295.12077 & 0.0168468 & 303.21005 & 0.1167592 \\
\hline & 295.02495 & 0.0185479 & 295.80709 & 0.0118080 & 304.51768 & 0.0430655 \\
\hline & 295.97566 & 0.0095416 & 296.45579 & 0.0074091 & 307.81809 & 0.0075612 \\
\hline & 296.96877 & 0.0275966 & $297 \cdot 47855$ & 0.0352343 & 308.77256 & 0.0052942 \\
\hline & $297 \cdot 52923$ & 0.0085666 & 297.87092 & 0.0078423 & 309.49283 & 0.0064490 \\
\hline & 298.18962 & 0.0091173 & 298.69746 & 0.0096848 & 310.22810 & 0.0030432 \\
\hline & 298.70402 & 0.0086144 & 299.13125 & 0.0135489 & 311.19617 & 0.0015608 \\
\hline & 298.87887 & 0.0022894 & 299.25610 & 0.0044325 & 311.42084 & 0.0148011 \\
\hline & 299.93712 & 0.0026914 & 300.41443 & 0.0014695 & 311.77485 & 0.0113018 \\
\hline & 300.86385 & 0.0094459 & 301.28190 & 0.0106421 & 313.00726 & 0.0068836 \\
\hline & $301 \cdot 37171$ & 0.0013593 & & & & \\
\hline & 301.42761 & 0.0015884 & & & & \\
\hline & 301.74722 & 0.0002432 & & & & \\
\hline \multirow{5}{*}{$\mathrm{A}^{\prime \prime}$} & 289.62475 & 0.0727809 & 290.61605 & 0.0790657 & 296.35349 & 0.1223200 \\
\hline & 296.09720 & 0.0062365 & 296.50456 & 0.0052737 & 308.11618 & 0.0059235 \\
\hline & 298.98543 & 0.0002213 & 299.41175 & 0.0002180 & 311.51217 & 0.0053391 \\
\hline & $299 \cdot 57645$ & 0.0033533 & 300.16639 & 0.0034155 & 311.84648 & 0.0001788 \\
\hline & 300.53061 & 0.0062865 & 300.96951 & 0.0065391 & 312.71161 & 0.0037940 \\
\hline \multicolumn{7}{|c|}{ Fluorine K-edge } \\
\hline \multirow{14}{*}{$\mathrm{A}^{\prime}$} & 692.39381 & 0.0055812 & 689.13205 & 0.0001470 & $708.5795^{1}$ & 0.0634556 \\
\hline & 692.98496 & 0.0141520 & 689.80849 & 0.0016355 & 712.29108 & 0.0048860 \\
\hline & 694.13204 & 0.0069800 & 690.45042 & 0.0016173 & 713.63387 & 0.0155450 \\
\hline & 694.45278 & 0.0142131 & 691.72799 & 0.0019225 & 714.53843 & 0.0041029 \\
\hline & $695 \cdot 74972$ & 0.0006074 & 692.05036 & 0.0123871 & 716.05922 & 0.0011578 \\
\hline & 696.30436 & 0.0011727 & 692.71695 & 0.0020241 & 716.42056 & 0.0040269 \\
\hline & 696.56018 & 0.0042773 & 692.88214 & 0.0017271 & 716.69113 & 0.0031824 \\
\hline & 697.00126 & 0.0022495 & 693.81329 & 0.0054768 & $717 \cdot 49741$ & 0.0004426 \\
\hline & 698.28514 & 0.0018959 & 694.48224 & 0.0016087 & 718.37478 & 0.0044433 \\
\hline & 698.54513 & 0.0068786 & 695.05454 & 0.0042343 & 718.79806 & 0.0041661 \\
\hline & 699.03809 & 0.0028710 & 695.76708 & 0.0092152 & & \\
\hline & 699.65976 & 0.0016427 & 696.13878 & 0.0013452 & & \\
\hline & 700.48445 & 0.0031028 & 697.13012 & 0.0080867 & & \\
\hline & 700.80757 & 0.0001553 & 697.26341 & 0.0023598 & & \\
\hline \multirow{6}{*}{$\mathrm{A}^{\prime \prime}$} & 690.61442 & 0.0133013 & 687.93265 & 0.0103972 & $709 \cdot 49003$ & 0.0279289 \\
\hline & 694.15522 & 0.0004964 & 690.37207 & 0.0000786 & 714.49475 & 0.0010644 \\
\hline & 696.55986 & 0.0062080 & 693.26534 & 0.0033027 & 716.32361 & 0.0068014 \\
\hline & 697.60493 & 0.0010132 & 693.84397 & 0.0041445 & 718.32323 & 0.0006738 \\
\hline & 698.76060 & 0.0000363 & 695.02717 & 0.0000211 & & \\
\hline & 700.93628 & 0.0005710 & $697 \cdot 41945$ & 0.0008202 & & \\
\hline
\end{tabular}


Table 12: $\mathrm{CF}_{2} \mathrm{O}$ : calculated eCVS-CCSD, eCVS-CC2 and eCVS-CCS oxygen, carbon and fluorine core-excitation energies $(\mathrm{eV})$ and oscillator strengths in the aug-cc-pCVTZ basis set.

\begin{tabular}{|c|c|c|c|c|c|c|}
\hline & \multicolumn{2}{|c|}{ CCSD } & \multicolumn{2}{|c|}{$\mathrm{CC}_{2}$} & \multicolumn{2}{|c|}{ CCS } \\
\hline \multicolumn{7}{|c|}{ Oxygen K-edge } \\
\hline \multirow{8}{*}{$A_{1}$} & 538.30317 & 0.0025985 & 536.12606 & 0.0015197 & $555 \cdot 57279$ & 0.0202999 \\
\hline & 540.43388 & 0.0055146 & 537.96868 & 0.0014546 & $557 \cdot 44788$ & 0.0316689 \\
\hline & 541.69100 & 0.0066264 & 539.21374 & 0.0059033 & 559.65975 & 0.0062236 \\
\hline & 542.52184 & 0.0002601 & 540.14441 & 0.0001350 & 560.63968 & 0.0000012 \\
\hline & 543.60375 & 0.0010352 & 540.85411 & 0.0004348 & & \\
\hline & $544 \cdot 55565$ & 0.0023579 & 542.57846 & 0.0017892 & & \\
\hline & & & 544.00357 & 0.0001652 & & \\
\hline & & & 544.65035 & 0.0000014 & & \\
\hline \multirow{4}{*}{$\mathrm{B}_{1}$} & 534.68935 & 0.0407918 & 534.59832 & 0.0354545 & 548.50736 & 0.0719610 \\
\hline & 540.22919 & 0.0015339 & 537.63594 & 0.0009842 & 558.40681 & 0.0018279 \\
\hline & 542.58293 & 0.0040666 & 540.26936 & 0.0049378 & 560.55954 & 0.0031818 \\
\hline & 544.96066 & 0.0012162 & 542.25882 & 0.0013375 & & \\
\hline \multirow{6}{*}{$\mathrm{B}_{2}$} & 540.45471 & 0.0065342 & 537.95901 & 0.0024630 & $557 \cdot 40398$ & 0.0387235 \\
\hline & 542.31182 & 0.0002219 & 539.89877 & 0.0000315 & 560.42949 & 0.0152497 \\
\hline & 543.61870 & 0.0236223 & 542.22230 & 0.0152995 & 560.70339 & 0.0025089 \\
\hline & & & 542.52240 & 0.0058118 & & \\
\hline & & & 543.29179 & 0.0019013 & & \\
\hline & & & 544.85778 & 0.0000010 & & \\
\hline \multicolumn{7}{|c|}{ Carbon K-edge } \\
\hline \multirow{6}{*}{$\mathrm{A}_{1}$} & 296.28199 & 0.0109573 & 297.61226 & 0.0098335 & 305.24621 & 0.0440540 \\
\hline & 299.04246 & 0.0109977 & 299.93623 & 0.0053104 & 310.36740 & 0.0160997 \\
\hline & $299 \cdot 37188$ & 0.0037384 & 300.36653 & 0.0124248 & 311.86893 & 0.0006219 \\
\hline & 301.25454 & 0.0000002 & 302.09806 & 0.0000068 & & \\
\hline & 301.54191 & 0.0059827 & 302.48931 & 0.0059962 & & \\
\hline & 303.43210 & 0.0050068 & 304.38447 & 0.0057456 & & \\
\hline \multirow{4}{*}{$\mathrm{B}_{1}$} & 292.28691 & 0.0861406 & 293.74057 & 0.0924445 & 298.58423 & 0.1335001 \\
\hline & 298.86607 & 0.0017576 & 299.73859 & 0.0011963 & 311.12491 & 0.0013903 \\
\hline & 301.51148 & 0.0000038 & 302.39623 & 0.0000097 & & \\
\hline & 303.31290 & 0.0030330 & 304.21423 & 0.0023722 & & \\
\hline \multirow{4}{*}{$\mathrm{B}_{2}$} & 298.98168 & 0.0567946 & 299.93015 & 0.0137345 & 305.78540 & 0.1551998 \\
\hline & $299 \cdot 37649$ & 0.0430463 & 300.46794 & 0.0857296 & & \\
\hline & 301.79483 & 0.0129094 & 302.70599 & 0.0213229 & & \\
\hline & 303.60924 & 0.0001017 & 304.38038 & 0.0002430 & & \\
\hline
\end{tabular}


Table 13: $\mathrm{CF}_{2} \mathrm{O}$ : calculated eCVS-CCSD, eCVS-CC2 and eCVS-CCS fluorine coreexcitation energies (eV) and oscillator strengths in the aug-cc-pCVTZ basis set.

\begin{tabular}{|c|c|c|c|c|c|c|}
\hline & \multicolumn{2}{|c|}{ CCSD } & \multicolumn{2}{|c|}{$\mathrm{CC}_{2}$} & \multicolumn{2}{|c|}{ CCS } \\
\hline \multicolumn{7}{|c|}{ Fluorine K-edge } \\
\hline \multirow{15}{*}{$\mathrm{A}_{1}$} & 692.37257 & 0.0104163 & 689.98183 & 0.0018020 & 708.27242 & 0.0440596 \\
\hline & 694.62819 & 0.0141310 & 691.85894 & 0.0008410 & 713.28674 & 0.0069022 \\
\hline & $695 \cdot 38607$ & 0.0068776 & 691.96115 & 0.0038558 & 714.88277 & 0.0228435 \\
\hline & 695.83799 & 0.0016392 & 693.04121 & 0.0109656 & 716.13399 & 0.0000265 \\
\hline & $697 \cdot 37298$ & 0.0000177 & 693.92286 & 0.0000007 & 717.67629 & 0.0031593 \\
\hline & 697.65933 & 0.0024297 & 694.20968 & 0.0008612 & 717.80189 & 0.0053190 \\
\hline & 698.27574 & 0.0007236 & 694.73842 & 0.0015425 & 718.53966 & 0.0024669 \\
\hline & 699.27432 & o.0050788 & 696.18647 & 0.0050738 & 719.06237 & 0.0000969 \\
\hline & 699.57408 & 0.0146471 & 696.53781 & 0.0054545 & 719.42327 & 0.0167716 \\
\hline & 700.19373 & 0.0004913 & 696.70115 & 0.0006166 & & \\
\hline & 700.38642 & 0.0023104 & 697.05198 & 0.0013915 & & \\
\hline & 700.66519 & 0.0009814 & 697.22238 & 0.0101090 & & \\
\hline & 701.14996 & 0.0010041 & $697 \cdot 70664$ & 0.0051520 & & \\
\hline & 701.68157 & 0.0001285 & 698.37509 & 0.0026472 & & \\
\hline & & & 699.20068 & 0.0014234 & & \\
\hline \multirow{7}{*}{$\mathrm{B}_{1}$} & 692.13018 & 0.0281370 & 689.75488 & 0.0212701 & 710.77954 & 0.0604176 \\
\hline & 695.12002 & 0.0032298 & 691.62041 & 0.0021071 & 715.27665 & 0.0032726 \\
\hline & $697 \cdot 70270$ & 0.0079339 & 694.47165 & 0.0055627 & 717.63192 & 0.0081407 \\
\hline & 698.62926 & o.0009675 & 694.95574 & 0.0033229 & 719.28716 & 0.0006422 \\
\hline & 699.80446 & 0.0026205 & 696.21333 & 0.0036041 & & \\
\hline & 701.88634 & 0.0007653 & 698.64502 & 0.0014083 & & \\
\hline & & & 699.47901 & 0.0023296 & & \\
\hline \multirow{15}{*}{$\mathrm{B}_{2}$} & 692.37218 & 0.0281370 & 689.98259 & 0.0049033 & 708.26768 & 0.0859477 \\
\hline & 694.62676 & 0.0032298 & 691.85777 & 0.0068608 & 713.28635 & 0.0108241 \\
\hline & $695 \cdot 38629$ & 0.0079339 & 691.96207 & 0.0000275 & 714.88193 & 0.0114900 \\
\hline & 695.83807 & 0.0009675 & 693.04093 & 0.0226416 & 716.13418 & 0.0001612 \\
\hline & $697 \cdot 37286$ & 0.0026205 & 693.92218 & 0.0001848 & 717.67599 & 0.0046686 \\
\hline & 697.65995 & 0.0007653 & 694.21055 & 0.0028238 & 717.80197 & 0.0004556 \\
\hline & 698.27598 & 0.0023190 & 694.73898 & 0.0030723 & 718.53979 & 0.0044674 \\
\hline & 699.27424 & 0.0059198 & 696.18615 & 0.0037143 & 719.06237 & 0.0004787 \\
\hline & & & 696.53728 & 0.0038642 & & \\
\hline & & & 696.70085 & 0.0003354 & & \\
\hline & & & 697.05205 & 0.0084438 & & \\
\hline & & & 697.22171 & 0.0030804 & & \\
\hline & & & 697.70703 & 0.0015348 & & \\
\hline & & & 698.37522 & 0.0089743 & & \\
\hline & & & 699.20016 & 0.0001727 & & \\
\hline
\end{tabular}


Table 14: CHOOH: calculated eCV-CCSD, eCVS-CC2 and eCVS-CCS oxygen coreexcitation energies $(\mathrm{eV})$ and oscillator strengths in the aug-cc-pCVTZ basis set.

\begin{tabular}{|c|c|c|c|c|c|c|}
\hline & \multicolumn{2}{|c|}{ CCSD } & \multicolumn{2}{|c|}{$\mathrm{CC}_{2}$} & \multicolumn{2}{|c|}{ CCS } \\
\hline \multicolumn{7}{|c|}{ Oxygen K-edge } \\
\hline \multirow{25}{*}{$\mathrm{A}^{\prime}$} & $537 \cdot 45588$ & 0.0024065 & 534.71465 & 0.0015093 & 552.50169 & 0.0628794 \\
\hline & $537 \cdot 53581$ & 0.0170360 & 535.05775 & 0.0004104 & 555.24379 & 0.0092051 \\
\hline & 538.14426 & 0.0012946 & $535 \cdot 96424$ & 0.0008826 & 555.63432 & 0.0591396 \\
\hline & 538.75643 & 0.0024811 & 536.00715 & 0.0008020 & $555 \cdot 98024$ & 0.0210784 \\
\hline & 539.62224 & 0.0038962 & 536.70638 & 0.0000143 & 556.58572 & 0.0370289 \\
\hline & $539 \cdot 74992$ & 0.0024445 & 537.08106 & 0.0023081 & 557.19784 & 0.0136323 \\
\hline & 540.23215 & 0.0076245 & $537 \cdot 32625$ & 0.0030905 & & \\
\hline & 540.46451 & 0.0079649 & $537 \cdot 49682$ & 0.0014192 & & \\
\hline & 540.47145 & 0.0005513 & $537 \cdot 77680$ & 0.0003059 & & \\
\hline & 540.76759 & 0.0096551 & 537.99353 & 0.0009114 & & \\
\hline & 540.81705 & 0.0014109 & 538.20410 & 0.0005760 & & \\
\hline & 541.03480 & 0.0023641 & 538.49067 & 0.0036069 & & \\
\hline & 541.08649 & 0.0004469 & 538.74377 & 0.0040162 & & \\
\hline & 541.93767 & 0.0018528 & 539.48835 & 0.0006859 & & \\
\hline & 542.14746 & 0.0025463 & $539 \cdot 49616$ & 0.0040730 & & \\
\hline & 542.35421 & 0.0011156 & $539 \cdot 55981$ & 0.0072057 & & \\
\hline & 542.67556 & 0.0035520 & 539.85261 & 0.0011808 & & \\
\hline & 542.68739 & 0.0048933 & 540.08899 & 0.0018594 & & \\
\hline & 543.27624 & 0.0158396 & 540.14038 & 0.0019870 & & \\
\hline & 544.19996 & 0.0014987 & 540.28882 & 0.0102479 & & \\
\hline & & & 541.38757 & 0.0000155 & & \\
\hline & & & 541.39502 & 0.0004653 & & \\
\hline & & & 541.71096 & 0.0014143 & & \\
\hline & & & 542.00812 & 0.0041130 & & \\
\hline & & & 542.72347 & 0.0016996 & & \\
\hline \multirow{12}{*}{$\mathrm{A}^{\prime \prime}$} & $533 \cdot 77008$ & 0.0388251 & 533.30631 & 0.0323501 & 547.86737 & 0.0701167 \\
\hline & $537 \cdot 56157$ & 0.0167675 & 535.86477 & 0.0000548 & 553.97590 & 0.0406467 \\
\hline & 538.83423 & 0.0005605 & 536.14063 & 0.0029904 & 557.26311 & 0.0009732 \\
\hline & 540.34543 & 0.0022372 & 537.84638 & 0.0052807 & & \\
\hline & 541.05496 & 0.0049849 & 538.31661 & 0.0000005 & & \\
\hline & 541.77758 & $0.001245^{2}$ & 538.88683 & 0.0000003 & & \\
\hline & 542.23692 & 0.0067683 & 539.97608 & 0.0000396 & & \\
\hline & 543.23374 & 0.0000409 & 540.32490 & 0.0053211 & & \\
\hline & 543.26561 & 0.0011188 & 540.84119 & 0.0022670 & & \\
\hline & $544 \cdot 55^{241}$ & 0.0000026 & 541.54516 & 0.0002933 & & \\
\hline & 544.97428 & 0.0000365 & 542.38326 & 0.0126185 & & \\
\hline & & & 542.92942 & 0.0007246 & & \\
\hline
\end{tabular}


Table 15: CHOOH: calculated eCV-CCSD, eCVS-CC2 and eCVS-CCS carbon coreexcitation energies $(\mathrm{eV})$ and oscillator strengths in the aug-cc-pCVTZ basis set.

\begin{tabular}{|c|c|c|c|c|c|c|}
\hline & \multicolumn{2}{|c|}{ CCSD } & \multicolumn{2}{|c|}{$\mathrm{CC}_{2}$} & \multicolumn{2}{|c|}{ CCS } \\
\hline \multicolumn{7}{|c|}{ Carbon K-edge } \\
\hline \multirow{10}{*}{$A^{\prime}$} & 293.64766 & 0.0153817 & 294.57469 & 0.0074860 & 303.69535 & 0.0774747 \\
\hline & 293.85172 & 0.0028639 & 294.79952 & 0.0072028 & 304.94314 & 0.0645220 \\
\hline & 295.09954 & 0.0023863 & 295.91847 & 0.0020578 & 306.85089 & 0.0151081 \\
\hline & 295.80931 & 0.0020723 & 296.68862 & 0.0013345 & $307 \cdot 59035$ & 0.0021282 \\
\hline & 296.45926 & 0.0053930 & 297.16479 & 0.0099545 & & \\
\hline & 296.57431 & 0.0107023 & $297 \cdot 37030$ & 0.0020277 & & \\
\hline & 296.76767 & 0.0070312 & 297.53641 & 0.0081024 & & \\
\hline & $297 \cdot 30523$ & 0.0095517 & 298.12966 & 0.0060369 & & \\
\hline & & & 299.12376 & 0.0212794 & & \\
\hline & & & 299.62999 & 0.0042169 & & \\
\hline \multirow{6}{*}{$\mathrm{A}^{\prime \prime}$} & $289 \cdot 35236$ & 0.0729441 & 290.79214 & 0.0779670 & 295.98391 & 0.1239255 \\
\hline & 295.03543 & 0.0044754 & 295.84556 & 0.0036128 & 307.29686 & 0.0042301 \\
\hline & 297.60316 & 0.0000029 & 298.37672 & 0.0000019 & & \\
\hline & 297.89002 & 0.0021570 & 298.82507 & 0.0019877 & & \\
\hline & & & & & 311.40539 & 0.0042791 \\
\hline & & & & & 313.33285 & 0.0003188 \\
\hline
\end{tabular}




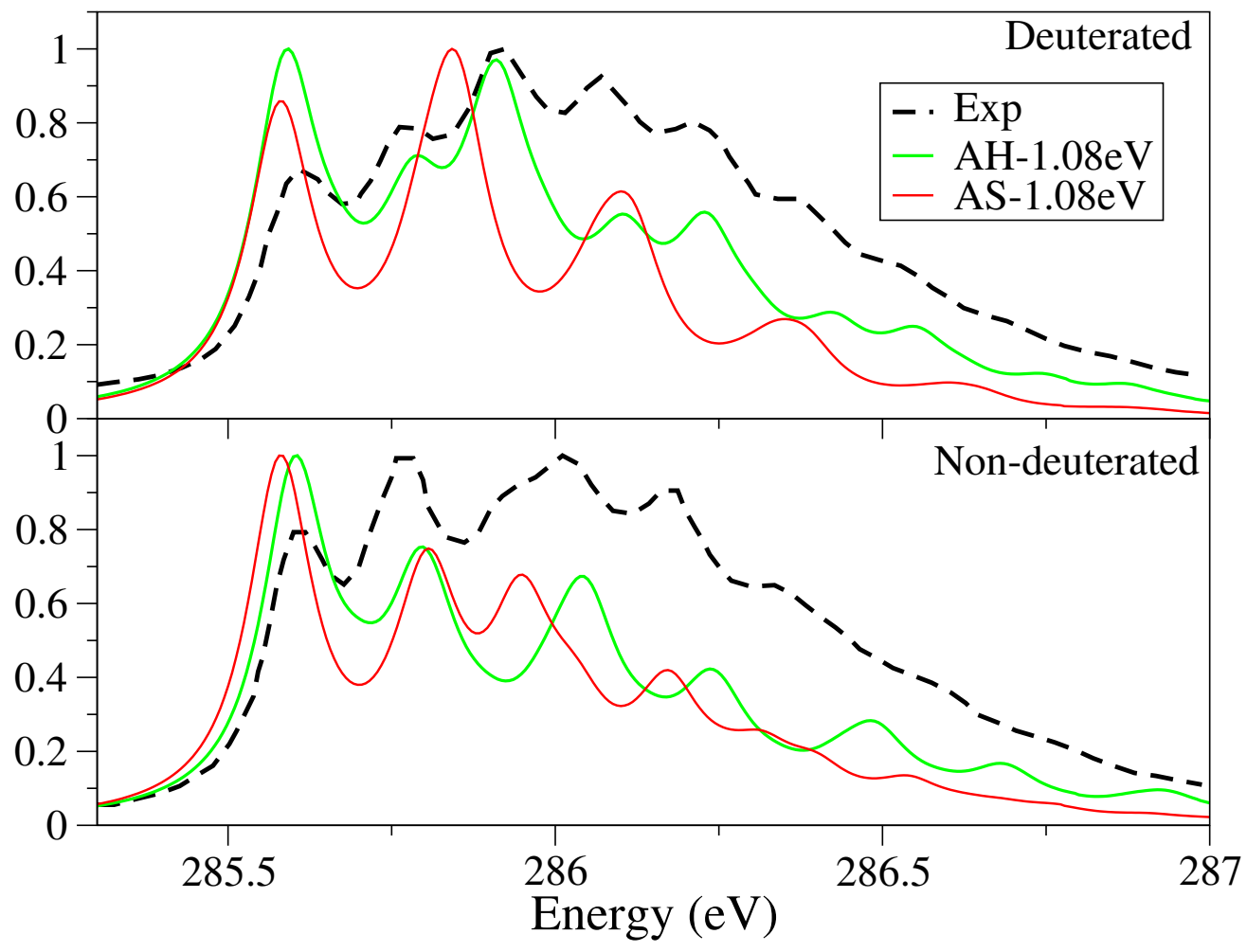

Figure 31: Formaldehyde: $\mathrm{AH}$ and AS simulations for the $\mathrm{C} 1 \mathrm{~s} \rightarrow \pi^{*}$ band at oK for non-deuterated formaldehyde, computed on the grounds of EOMCCSD PES, adopting a Lorentzian broadening of HWHM=0.06 eV, and their comparison to experiments [219]. Note that the simulated spectra are red-shifted by $1.08 \mathrm{eV}$ so as to match the position of the experimental bands. 
Table 16: Formaldehyde: geometrical parameters in different stationary points of the ground and carbon and oxygen K-edge core-excitation states.

\begin{tabular}{ccccc}
\hline Parameter & Ground & C K-edge & O K-edge(planar) & O K-edge(non-planar) \\
\hline $\mathrm{d}(\mathrm{C}=\mathrm{O}) / \AA$ & 1.202 & 1.266 & 1.296 & 1.296 \\
$\mathrm{~d}(\mathrm{C}-\mathrm{H}) / \AA$ & 1.100 & 1.023 & 1.072 & 1.072 \\
$\mathrm{a}(\mathrm{H}-\mathrm{C}-\mathrm{H}) / \mathrm{deg}$ & 116.6 & 119.4 & 126.9 & 126.6 \\
\hline
\end{tabular}




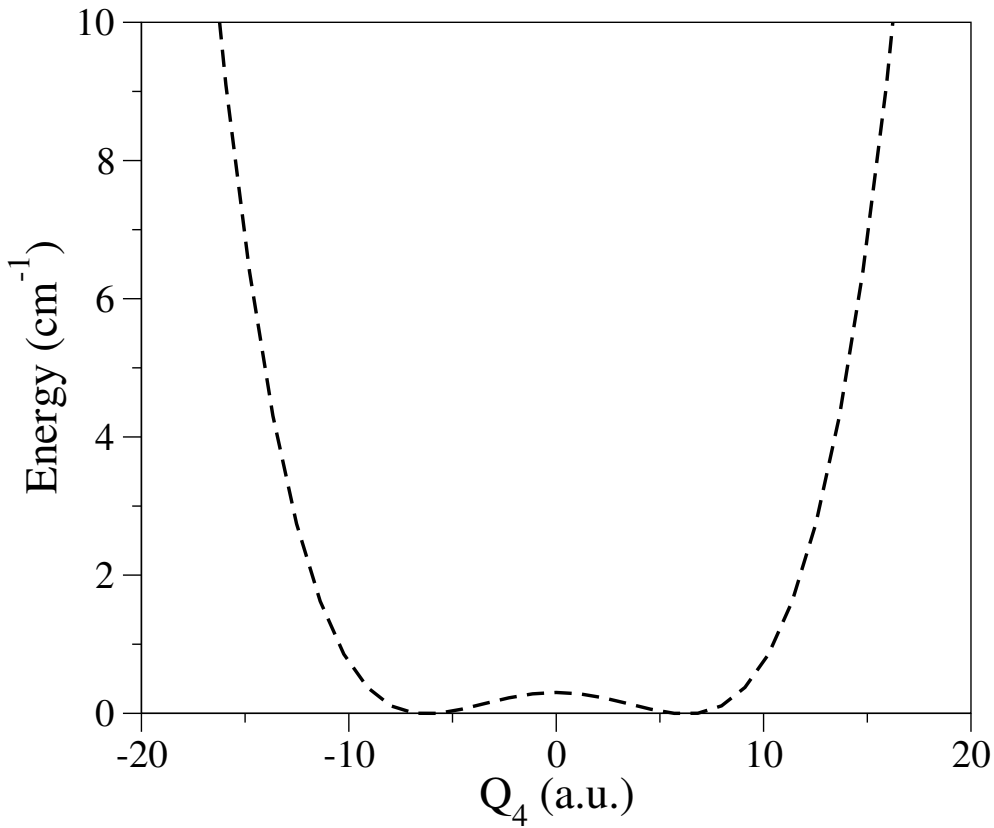

Figure 32: Energy scan along mode $4\left(\mathrm{Q}_{4}\right.$, out of plane-bending $)$ in the $\mathrm{O} 1 \mathrm{~s} \rightarrow \pi^{*}$ state.

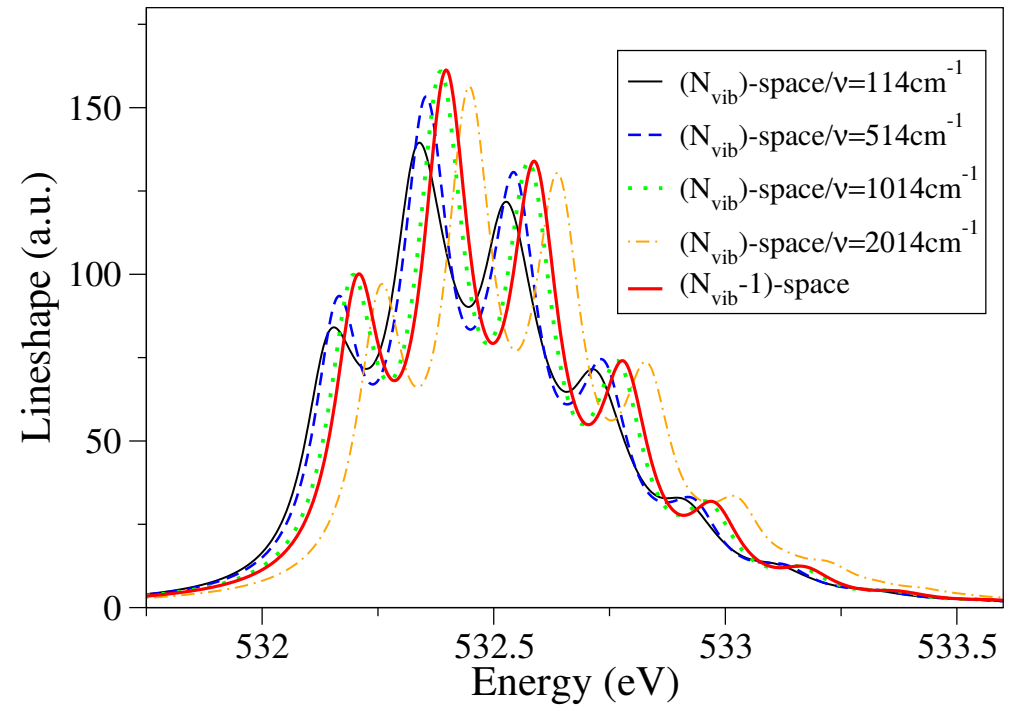

Figure 33: Simulation of the O $1 \mathrm{~s} \rightarrow \pi^{*}$ band at oK, on the grounds of EOM-CCSD PES adopting a Lorentzian broadening of $\mathrm{HWHM}=0.06 \mathrm{eV}$. The spectra are computed at the $\mathrm{C}_{2 v}$ stationary point in the excited state, but substituting the corresponding imaginary frequency $\left(v_{4}^{(E S)}\right)$ by a range of arbitrary values from 114 to $2014 \mathrm{~cm}^{-1}$. 
Table 17: $\mathrm{CHOOH}$ : calculated eCV-CCSD, eCVS-CC2 and eCVS-CCS oxygen and carbon core-excitation energies (eV) and oscillator strengths in the aug-ccpCVTZ basis set.

\begin{tabular}{|c|c|c|c|c|c|c|}
\hline & \multicolumn{2}{|c|}{ CCSD } & \multicolumn{2}{|c|}{$\mathrm{CC}_{2}$} & \multicolumn{2}{|c|}{ CCS } \\
\hline \multicolumn{7}{|c|}{ Oxygen K-edge } \\
\hline \multirow{25}{*}{$A^{\prime}$} & $537 \cdot 45588$ & 0.0024065 & 534.71465 & 0.0015093 & 552.50169 & 0.0628794 \\
\hline & $537 \cdot 53581$ & 0.0170360 & 535.05775 & 0.0004104 & 555.24379 & 0.0092051 \\
\hline & 538.14426 & 0.0012946 & 535.96424 & 0.0008826 & 555.63432 & 0.0591396 \\
\hline & 538.75643 & 0.0024811 & 536.00715 & 0.0008020 & 555.98024 & 0.0210784 \\
\hline & 539.62224 & 0.0038962 & 536.70638 & 0.0000143 & 556.58572 & 0.0370289 \\
\hline & 539.74992 & 0.0024445 & 537.08106 & 0.0023081 & 557.19784 & 0.0136323 \\
\hline & 540.23215 & 0.0076245 & $537 \cdot 32625$ & 0.0030905 & & \\
\hline & 540.46451 & 0.0079649 & $537 \cdot 49682$ & 0.0014192 & & \\
\hline & 540.47145 & 0.0005513 & $537 \cdot 77680$ & 0.0003059 & & \\
\hline & 540.76759 & 0.0096551 & 537.99353 & 0.0009114 & & \\
\hline & 540.81705 & 0.0014109 & 538.20410 & 0.0005760 & & \\
\hline & 541.03480 & 0.0023641 & 538.49067 & 0.0036069 & & \\
\hline & 541.08649 & 0.0004469 & 538.74377 & 0.0040162 & & \\
\hline & 541.93767 & 0.0018528 & $539 \cdot 48835$ & 0.0006859 & & \\
\hline & 542.14746 & 0.0025463 & $539 \cdot 49616$ & 0.0040730 & & \\
\hline & 542.35421 & 0.0011156 & $539 \cdot 55981$ & 0.0072057 & & \\
\hline & 542.67556 & 0.0035520 & 539.85261 & 0.0011808 & & \\
\hline & 542.68739 & 0.0048933 & 540.08899 & 0.0018594 & & \\
\hline & 543.27624 & 0.0158396 & 540.14038 & 0.0019870 & & \\
\hline & 544.19996 & 0.0014987 & 540.28882 & 0.0102479 & & \\
\hline & & & $541 \cdot 38757$ & 0.0000155 & & \\
\hline & & & 541.39502 & 0.0004653 & & \\
\hline & & & 541.71096 & 0.0014143 & & \\
\hline & & & 542.00812 & 0.0041130 & & \\
\hline & & & 542.72347 & 0.0016996 & & \\
\hline \multirow{12}{*}{$\mathrm{A}^{\prime \prime}$} & 533.77008 & 0.0388251 & $533 \cdot 30631$ & 0.0323501 & 547.86737 & 0.0701167 \\
\hline & $537 \cdot 56157$ & 0.0167675 & 535.86477 & 0.0000548 & 553.97590 & 0.0406467 \\
\hline & 538.83423 & 0.0005605 & 536.14063 & 0.0029904 & 557.26311 & 0.0009732 \\
\hline & 540.34543 & 0.0022372 & 537.84638 & 0.0052807 & & \\
\hline & 541.05496 & 0.0049849 & 538.31661 & 0.0000005 & & \\
\hline & $541.7775^{8}$ & 0.0012452 & 538.88683 & 0.0000003 & & \\
\hline & 542.23692 & 0.0067683 & $539 \cdot 97608$ & 0.0000396 & & \\
\hline & 543.23374 & 0.0000409 & 540.32490 & 0.0053211 & & \\
\hline & 543.26561 & 0.0011188 & 540.84119 & 0.0022670 & & \\
\hline & $544 \cdot 55^{241}$ & 0.0000026 & 541.54516 & 0.0002933 & & \\
\hline & $544 \cdot 97428$ & 0.0000365 & 542.38326 & 0.0126185 & & \\
\hline & & & 542.92942 & 0.0007246 & & \\
\hline \multicolumn{7}{|c|}{ Carbon K-edge } \\
\hline \multirow{9}{*}{$A^{\prime}$} & 293.64766 & 0.0153817 & 294.57469 & 0.0074860 & 303.69535 & o.0774747 \\
\hline & 293.85172 & 0.0028639 & 294.79952 & 0.0072028 & 304.94314 & 0.0645220 \\
\hline & 295.09954 & 0.0023863 & $295 \cdot 91847$ & 0.0020578 & 306.85089 & 0.0151081 \\
\hline & 295.80931 & 0.0020723 & 296.68862 & 0.0013345 & $307 \cdot 59035$ & 0.0021282 \\
\hline & 296.45926 & 0.0053930 & 297.16479 & 0.0099545 & & \\
\hline & 296.57431 & 0.0107023 & $297 \cdot 37030$ & 0.0020277 & & \\
\hline & 296.76767 & 0.0070312 & 297.53641 & 0.0081024 & & \\
\hline & $297 \cdot 30523$ & 0.0095517 & 298.12966 & 0.0060369 & & \\
\hline & & & 299.12376 & 0.0212794 & & \\
\hline
\end{tabular}




\section{Part III}

\section{SOLID SYSTEMS}

Part III deals with the experimental results for solid oxides, starting from s- and p-electron oxides presented in Chapter 4 and Chapter 5. Examples of theoretical simulations for these oxides are introduced in order to show how accurate a DFT description can be in case of $\mathrm{s}$ and $\mathrm{p}$ electron overlap. The discussion moves in Chapter 5 on the alkaline earth metal oxides, where a ground state analysis combined with the simulation of the oxygen $\mathrm{K}$ edge absorption spectra is presented in order to clarify discrepancies between the experimental and the theoretical data and further investigate the nature of the metal-oxygen bond. In Chapter 6 we discuss the general analysis of the $3 \mathrm{~d}$ transition metal oxides including discussions of the crystal field effect, trends in oxidation state and covalency. In addition to the general concepts, we give a systematic overview of the oxygen K edges element by element, for the $\mathrm{s}^{-}, \mathrm{p}^{-}, \mathrm{d}-\mathrm{and}$-electron systems. Part III is based on the papers: F. Frati, M. O.J.Y. Hunault, and F.M.F. de Groot. "Oxygen K-edge X-ray Absorption Spectra." Chemical Reviews 120.9 (2020): 4056-4110 and Density functional study of oxygen K edge $x$-ray absorption and electronic structure of alkaline earth metal oxides (in preparation) 



\section{S AND P-OXIDES}

\subsection{ALKALI METAL OXIDES}

The alkali metals lithium, sodium, potassium, rubidium and cesium form a number of different oxides.

Taking lithium as example, lithium forms respectively the oxide $\mathrm{Li}_{2} \mathrm{O}$, the peroxide $\mathrm{Li}_{2} \mathrm{O}_{2}$, the superoxide $\mathrm{LiO}_{2}$. The superoxide $\mathrm{MO}_{2}$ can be considered as a $\mathrm{M}^{+}$ion combined with a negatively charged oxygen molecule $\mathrm{O}_{2}^{-}$. The molecular orbitals of $\mathrm{O}_{2}^{-}$are similar to those of $\mathrm{O}_{2}$ with one more electron occupying the anti-bonding $\pi^{*}$ orbital leaving only one hole. The extra charge on the oxygen molecule makes it easier to excite a is core electron to the empty orbitals. Figure 34 shows the spectra of superoxide $\mathrm{MO}_{2}(\mathrm{M}=\mathrm{Li}$, $\mathrm{K}, \mathrm{Cs}$ ) compared to the molecular $\mathrm{O}_{2}$ [159]. The spectra of the superoxides show a first peak assigned to the $\pi^{*}$ orbital and two peaks assigned the $\sigma^{*}$ orbital and the ionisation potential. The three features shift towards lower energy for the different superoxides [159].

Pedio et al. also measured the superoxide $\mathrm{KO}_{2}$ [271]. The experimental spectrum is equivalent to that shown in Figure 34, though with a $\pi^{*}$ peak at 531.0 $\mathrm{eV}$ instead of 529.0 eV. Kang et al. reproduce the oxygen $\mathrm{K}$ edge of $\mathrm{KO}_{2}$ from band structure calculations using Wien2K [272].

The $\mathrm{M}_{2} \mathrm{O}$ oxides $(\mathrm{M}=\mathrm{Li}, \mathrm{Na}, \mathrm{K}, \mathrm{Rb})$ crystallise in the cubic antifluorite structure, $\mathrm{Cs}_{2} \mathrm{O}$ crystallises in hexagonal anti- $\mathrm{CdCl}_{2}$ structure. Alkaline ions occur mainly as cations $\mathrm{M}^{+}$and have rather weak bonds.

Lithium oxides are an important compounds of many battery systems. This includes lithium-air batteries, where the reactions between lithium and oxygen play an important role. The reversible oxidation of lithium at the anode and reduction of oxygen at the cathode are important parameters that influence the energy capacity of the battery system. $\mathrm{Li}_{2} \mathrm{O}, \mathrm{Li}_{2} \mathrm{O}_{2}$ have been studied as key components forming at the solid electrolyte interphase in lithium batteries and their evolution was studied with the oxygen $\mathrm{K}$ edge by Qiao et al. [273] Figure 34 shows the spectra of $\mathrm{Li}_{2} \mathrm{O}$ and $\mathrm{Li}_{2} \mathrm{O}_{2} \cdot \mathrm{Li}_{2} \mathrm{O}_{2}$ shows a first peak assigned to the $\sigma^{*}$ orbital of the $\mathrm{O}-\mathrm{O}$ bond. It is found that both $\mathrm{Li}_{2} \mathrm{O}_{2}$ and $\mathrm{Li}_{2} \mathrm{CO}_{3}$ evolve towards $\mathrm{Li}_{2} \mathrm{O}$ under the soft $\mathrm{x}$-ray irradiation, where $\mathrm{Li}_{2} \mathrm{CO}_{3}$ is very sensitive [273]. 


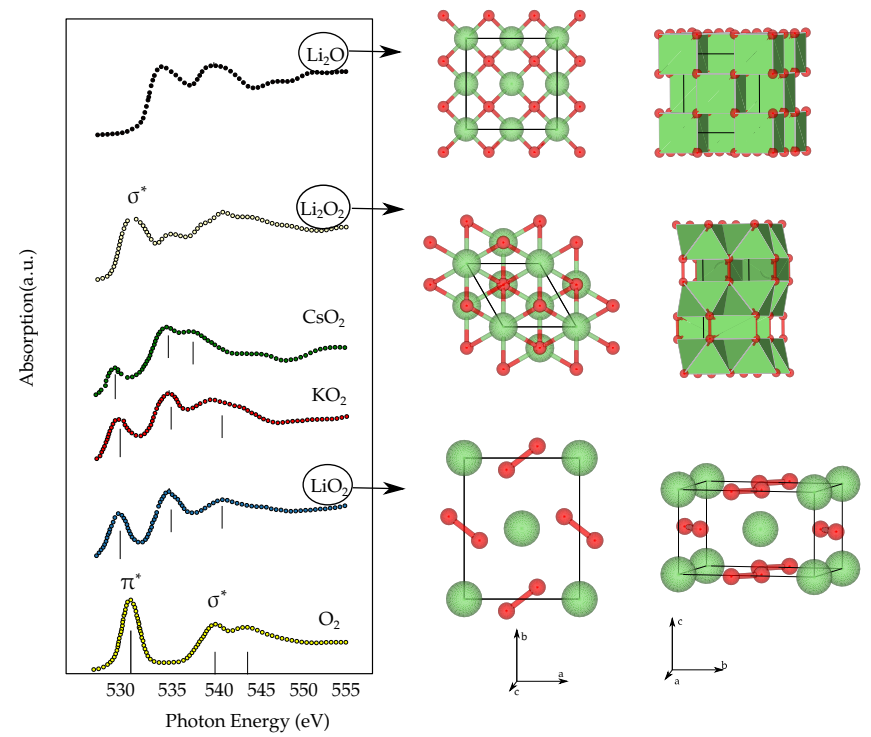

Figure 34: Oxygen $\mathrm{K}$ edge spectra of the oxide $\mathrm{Li}_{2} \mathrm{O}$, the peroxide $\mathrm{Li}_{2} \mathrm{O}_{2}$ [273] and the superoxides $\mathrm{LiO}_{2}, \mathrm{CsO}_{2}, \mathrm{KO}_{2}$ [159] compared to $\mathrm{O}_{2}$. The $\sigma$ and $\pi$ character of the antibonding states are indicated.

\subsection{OXIDES WITH P-ELECTRON SYSTEMS}

\subsubsection{General considerations}

Solid oxides of p-electron element systems form insulators that can be well described by band structure calculations. One of the major issues in the study of p-element oxides is the determination of the atomic structure of the solid. While the average crystalline structure can be determined by $x-$ ray diffraction, variations of the local environment of each atom in the solid can be studied with x-ray absorption using EXAFS, where the EXAFS experiments are usually performed on the hard x-ray cation XAS spectra. Furthermore, amorphous materials do not have a periodic structure and XAS is a key method to determine the local structure of the atoms. The high covalency of the cation-oxygen bond has been a motivation for the investigation of the geometric and electronic structures from the oxygen point of view.

The oxygen $2 p$ orbitals form bonding and anti-bonding bands with the cation $\mathrm{ns}$ and $\mathrm{np}$ orbitals. The oxygen $\mathrm{K}$ edge maps the empty states and shows the cation combined sp-bands. Figure 35 compares the spectra of quartz and rutile polymorphs of $\mathrm{SiO}_{2}$ and $\mathrm{GeO}_{2}$ and rutile $\mathrm{SnO}_{2}$. The spectra show the dependence of the oxygen $\mathrm{K}$ edge on the crystalline structure, the bond angles and the resulting consequences for the mixing of the oxygen $2 p$ orbitals with the ns, $n p$ and nd orbitals.

In the quartz structure, the cation is in four-fold tetrahedral coordination 


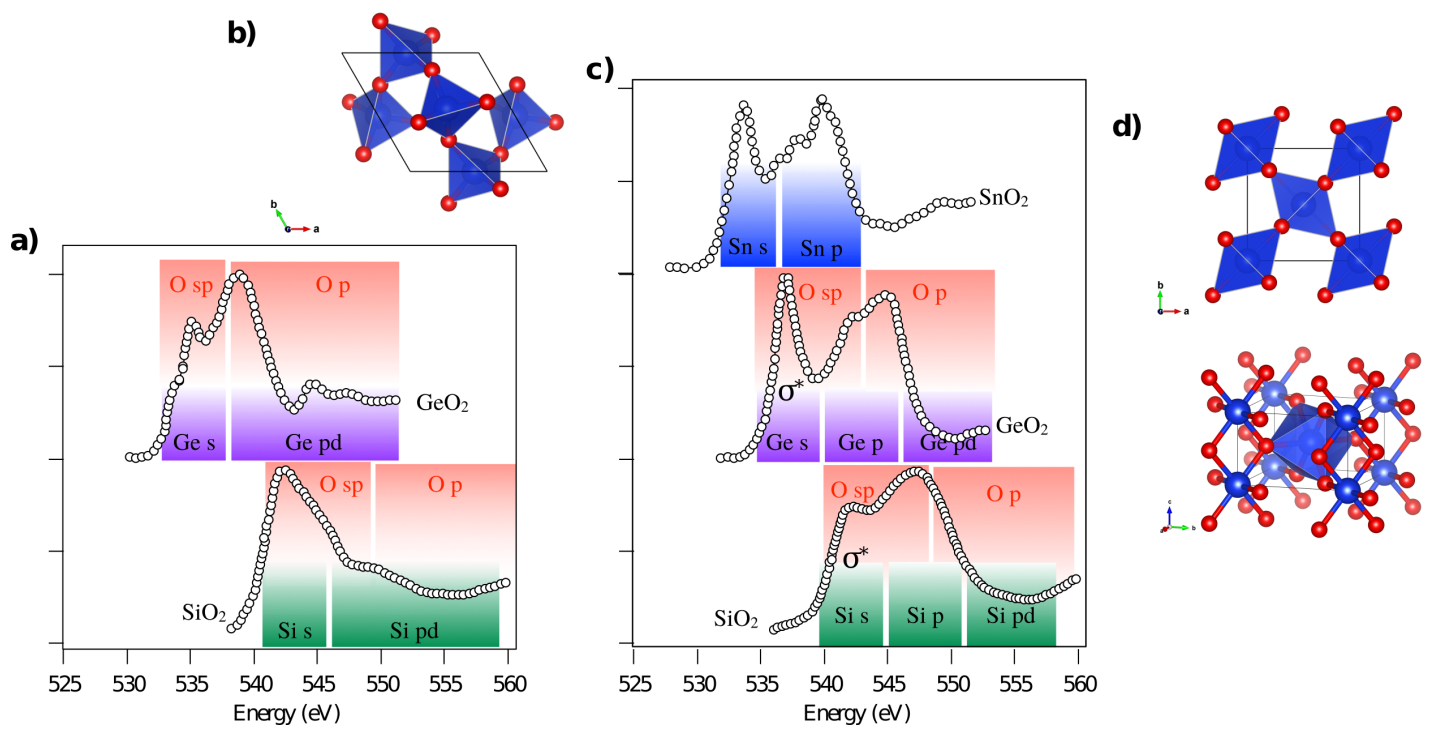

Figure 35: a) Oxygen $\mathrm{K}$ edge spectra of $\mathrm{SiO}_{2}$ [274] and $\mathrm{GeO}_{2}$ [275] in quartz structure (b) and c) oxygen $\mathrm{K}$ edge spectra of $\mathrm{SiO}_{2}$ [274], $\mathrm{GeO}_{2}$ [275] and $\mathrm{SnO}_{2}[276]$ in rutile structure (d). The nature of the final states are assigned based on the DOS calculations.

and the oxygen is two-fold coordinated. In the rutile structure, the cation is in six-fold elongated octahedral coordination and the oxygen is three-fold coordinated. The multiple scattering calculations [277, 278], later confirmed by DFT calculations [279], have allowed to assign the oxygen $\mathrm{K}$ edge features in $\alpha$-quartz $\mathrm{SiO}_{2}$ : the main intense peak at the edge is assigned to the $\mathrm{O} 2 \mathrm{sp}$ hybridized with $\mathrm{Si} 3 \mathrm{~s}$ followed by features assigned to transitions to O $2 p$ hybridized with Si $3 p d$ states split by the crystal field. We note that the cation tetrahedral coordination allows the cation pd state mixing. The spectral shape of quartz- $\mathrm{GeO}_{2}$ is drastically different as a consequence of the different cation-O-cation bond angle (from $143.7^{\circ}$ in $\mathrm{SiO}_{2}$ to $130^{\circ}$ in $\mathrm{GeO}_{2}$ ) on the orbital overlap [280]. However, in both cases, the general final state assignment is similar for $\mathrm{SiO}_{2}$ and $\mathrm{GeO}_{2}$.

Contrary to the quartz compounds, the oxygen $\mathrm{K}$ edge spectra of $\mathrm{SiO}_{2}, \mathrm{GeO}_{2}$ and $\mathrm{SnO}_{2}$ in rutile structure, Figure 35c),show similar shapes: a first sharp peak followed by a broader structured feature. In the case of $\mathrm{SiO}_{2}$, these features are not as well separated as in $\mathrm{GeO}_{2}$ and $\mathrm{SnO}_{2}$.

According to the calculated p-DOS [280, 281], the first sharp feature corresponds to the $\mathrm{O}$ 2sp anti-bonding $\sigma^{*}$ states hybridized with the empty cation ns states ( $\mathrm{Si}: \mathrm{n}=3$, Ge: $\mathrm{n}=4$ and $\mathrm{Sn}: \mathrm{n}=5$ ).

The following broad feature is assigned to $\mathrm{O}$ 2sp states and $\mathrm{O} 2 \mathrm{p}$ states hybridized with the cation $n p$ states. For Ge and $S n$, the contribution at higher 
energy from (n-1)d states is also found.

The p-DOS calculations shows that the missing mixing of the cation $\mathrm{p}$ and $\mathrm{d}$ states as not allowed in the octahedral symmetry. The spectra of the rutile compounds shift to low energy along the series in agreement with the decrease of their respective band gaps.

\subsection{OVERVIEW OF P-ELEMENT OXIDES}

Boron oxides: Boron and borate oxides are formed by two different struc-

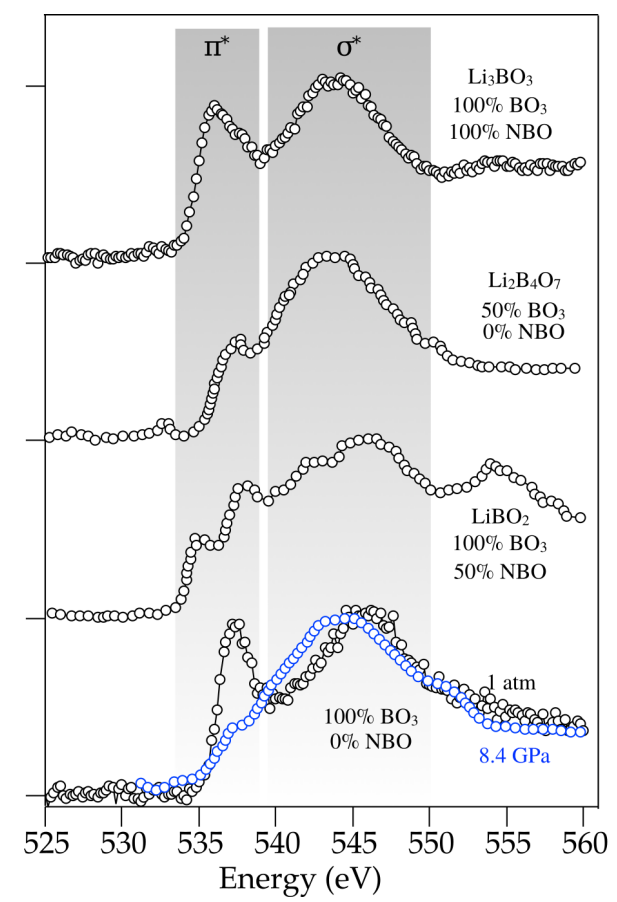

Figure 36: Oxygen $\mathrm{K}$ edge spectra of amorphous $\mathrm{B}_{2} \mathrm{O}_{3}$ at 1 atm [282] and 8.4GPa, $\mathrm{LiBO}_{2}$ [282], $\mathrm{Li}_{2} \mathrm{~B}_{4} \mathrm{O}_{7}$ [282] and $\mathrm{Li}_{3} \mathrm{BO}_{3}$ [282], with the fraction of $\mathrm{BO}_{3}$ units and non-brindging oxygens (NBO) indicated. The formation of NBO induces the shift of the $\pi^{*}$ feature to lower energy and the the conversion from $\mathrm{BO}_{3}$ to $\mathrm{BO}_{4}$ induces the decrease of its intensity.

tural units depending on the nature and content of alkali cations and/or the temperature and pressure conditions: planar $\mathrm{BO}_{3}$ triangles or $\mathrm{BO}_{4}$ tetrahedrons. The coordination of oxygen can be 2 -fold bridging oxygens or 1 -fold coordinated non-bridging oxygens.

In glasses, the addition of alkalis induces the transformation from $\mathrm{BO}_{3}$ to $\mathrm{BO}_{4}$ and a higher connectivity between the $\mathrm{B}$ units. Further increase of the alkali content induces the depolymerization of the glass or crystal network [283]. The determination of the $\mathrm{B}$ and $\mathrm{O}$ coordination is thus a critical 
parameter to understand the properties of borate crystals and glasses in various temperature and pressure conditions. For these systems, the low penetration depth of soft-X-rays and surface sensitivity have been a serious limitation for their investigation, especially because of their particular hygroscopicity. IXS has been used as a good solution to circumvent these limitations. Lelong et al. have investigated of the oxygen $\mathrm{K}$ edge XAS via IXS in a series of crystalline lithium borates [282] and in comparison with glasses [283].

Figure 36 shows the oxygen $\mathrm{K}$ edge of selected boron oxides. The spectra show two main features: the first $5 \mathrm{eV}$ of the oxygen $\mathrm{K}$ edge spectra is assigned to $\pi^{*}$ overlap between $\mathrm{O} 2 p$ and $\mathrm{B} 2 p$ while the next $10 \mathrm{eV}$ range is assigned to $\sigma^{*} \mathrm{O}-\mathrm{B}$ overlap. DFT calculations have further allowed to distinguish the contribution of bridging and non-bridging oxygens according to the energy position of the $\pi^{*}$-peak, which shifts to lower energy in the case of non-bridging oxygens as observed when comparing amorphous $\mathrm{B}_{2} \mathrm{O}_{3}$ and $\mathrm{Li}_{3} \mathrm{BO}_{3}$. Comparison between crystals and glasses has allowed a further understanding of the structural variations in lithium borate glasses with the chemical composition.

Lee and al. have studied $\mathrm{v}-\mathrm{B}_{2} \mathrm{O}_{3}$ glass at high pressure using IXS up to 8.4 GPa. Figure 36 shows the decrease of the $\pi^{*}$-peak related to boroxol rings, i.e. planar structure formed by three $\mathrm{BO}_{3}$ units, upon increasing the pressure together with the increase of the B coordination from 3- to 4-fold suggesting that the unpaired Op orbital gets involved in forming a $\sigma^{*}$ bond with an other B resulting in a 3-fold oxygen [284].

Carbonates: Carbonates are important compounds for the carbon cycle. Carbonate rocks result from the mineralisation of the $\mathrm{CO}_{2}$ from the atmosphere. Figure 37 shows the oxygen $\mathrm{K}$ edge of $\mathrm{BaCO}_{3}, \mathrm{Li}_{2} \mathrm{CO}_{3}, \mathrm{CaCO}_{3}$, $\mathrm{Na}_{2} \mathrm{CO}_{3}$. The oxygen $\mathrm{K}$ edge XAS spectra of carbonate compounds are dominated by the oxygen antibonding states from the $\mathrm{CO}_{3}^{-2}$ units, similarly to the $\mathrm{BO}_{3}$ units for boron (see above). These planar triangular units favour the formation of in-plane $\sigma$ covalent bonds involving $s p^{2}$ mixing. The first intense and sharp peak is assigned to the $\pi *$ anti-bonding state from the $\mathrm{C}=\mathrm{O}$ bonds resulting from out-of-plane $\pi$ bonds involving only $p$ states. The second part of the spectra corresponds to the $\sigma *$ anti-bonding state. Calcium carbonate, $\mathrm{CaCO}_{3}$, is an important biomineral occurring in shells and corals. $\mathrm{CaCO}_{3}$ occurs in three different structures, calcite, aragonite, and vaterite; In addition there are hydrated and amorphous structures.

$\mathrm{DeVol}$ et al. studied the oxygen $\mathrm{K}$ edge of these forms of $\mathrm{CaCO}_{3}$, using the polarization dependence of single crystals [286]. The experiments are simu- 


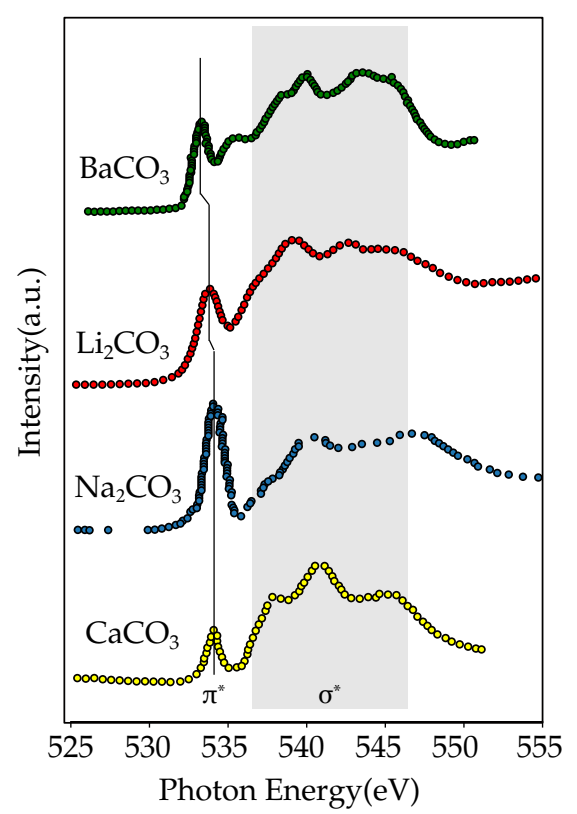

Figure 37: Oxygen $\mathrm{K}$ edge spectra of carbonates: $\mathrm{BaCO}_{3}$ [285], $\mathrm{Li}_{2} \mathrm{CO}_{3}$ [159], $\mathrm{CaCO}_{3}$ [286], $\mathrm{Na}_{2} \mathrm{CO}_{3}$ [287], all showing the $\pi *$ anti-bonding state from the $\mathrm{C}=\mathrm{O}$ bonds of the $\mathrm{CO}_{3}^{-2}$ units.

lated with FEFF calculations. Despite similar isotropic spectral shapes, the $\mathrm{O} \mathrm{K}$ edge polarisation dependance varies with the crystal structure of the polymorph. The polarization dependent contrast is used to study natural species (shells and tunic spicules) with x-ray spectral microscopy.

De Clermont Gallerande et al. analysed the monopole and dipole contributions to the oxygen $\mathrm{K}$ edge spectra of $\mathrm{Li}_{2} \mathrm{CO}_{3}$ measured with IXS, allowing to separate the $\pi$ and $\sigma$ anti-bonding states. The oxygen $\mathrm{K}$ edge of $\mathrm{Na}_{2} \mathrm{Si}_{2} \mathrm{O}_{5}$ shows a sharp peak at $533 \mathrm{eV}$ that is assigned to $\mathrm{Na}_{2} \mathrm{CO}_{3}$ adsorbed at the surface [288]. $\mathrm{Na}_{2} \mathrm{CO}_{3}$ and $\mathrm{NaHCO}_{3}$ have been measured by Espinal et al. [287]. They are used as references to study the interaction of $\mathrm{CO}_{2}$ with amine groups. Recently the oxygen $\mathrm{K}$ edge of barium carbonates and nitrates have been studied, in comparison with the binary oxide.

Karshoglu et al. measured the oxygen $\mathrm{K}$ edge of $\mathrm{BaO}, \mathrm{Ba}(\mathrm{OH})_{2}, \mathrm{BaCO}_{3}$, and $\mathrm{Ba}\left(\mathrm{NO}_{3}\right)_{2}$, prepared in-situ as thin films [289]. In this study they analysed the sensitivity of these materials and they discussed some published spectra of $\mathrm{BaO}$ that turned out to have a different composition as claimed in the original papers.

Aluminum oxides: Aluminium is the third most abundant element of the earth crust [290] after oxygen and silicon and is present as trivalent cations in various biological and mineralogical forms. The oxygen $\mathrm{K}$ edge of $\alpha-\mathrm{Al}_{2} \mathrm{O}_{3}$ (corundum struture), $\mathrm{MgO}$ and $\mathrm{MgAl}_{2} \mathrm{O}_{4}$ (spinel strucutre) are 
compared with their calculated projected DOS by Kostlmeier et al. [291]. Kaneko et al. study the difference between the oxygen $\mathrm{K}$ edge of the center of a $\mathrm{Al}_{2} \mathrm{O}_{3}$ grain with the grain boundary [292]. The features correspond to the anti-bonding $\mathrm{O}$ states hybridized with $\mathrm{Al}$ 3s, $\mathrm{p}$ and sp states [293]. Henderson et al. studied the oxygen $\mathrm{K}$ edge of a series of crystalline calcium aluminates [294] followed by the study of a series of $\mathrm{CaO}-\mathrm{Al}_{2} \mathrm{O}_{3}-\mathrm{SiO}_{2}$ mixed glasses [293].

The oxygen $\mathrm{K}$ edge spectra of the glasses reveal the contribution of both $\mathrm{Ca}$ and $\mathrm{Al}$ hybridized states and the coordination change of $\mathrm{Ca}$ as well as the degree of polymerisation of $\mathrm{AlO}_{4}$ tetrahedral units. The oxygen $\mathrm{K}$ edges of $\mathrm{Cu}-\mathrm{Al}-\mathrm{O}$ thin films have been compared with copper oxide and $\mathrm{Al}_{2} \mathrm{O}_{3}$ references [295].

The oxygen $\mathrm{K}$ edge of crystalline $\alpha-\mathrm{Al}_{2} \mathrm{O}_{3}$ has been compared for different shapes, respectively microparticles, nanowires and nanotubes [296].

\section{Silicon oxides:}

$\mathrm{SiO}_{2}$ is the first most abundant oxide of the earth crust [290] mainly as part of the mineral species like silica and silicates. $\mathrm{O} K$ edge have been used to investigate the atomic structure of crystalline and amorphous silica and silicates.

Marcelli et al. showed the sensitivity of the oxygen $\mathrm{K}$ edge on the Si-O-Si bond angle in glasses [297], which stimulated intense efforts to the development of accurate modelling of the oxygen $\mathrm{K}$ edge using multiple scattering approaches $[277,297,298]$ and later followed by band structure calculations $[274,279]$. The experimental oxygen $\mathrm{K}$ edge spectra of silica crystal polymorphs were investigated by $\mathrm{Wu}$ et al. [277].The influence of the chemical composition of silicates glasses on the structure was investigated [275, 299-301]. The interpretation of the spectra is more complex when considering silicate glasses with also calcium, aluminium and titanium. The oxygen $\mathrm{K}$ edge spectra reveal the bonding with the different cations but is also influenced by the coordination geometry changes. Another important aspect of glass structure is the degree of polymerisation of the glass network. The addition of alkali cations to amorphous $\mathrm{SiO}_{2}$ induces the depolymerisation of the $\mathrm{SiO}_{4}$ network and formation of non-brigding oxygens $(\mathrm{O}$ bond to a single $\mathrm{Si}$ ). It has been shown with the support of full multiple scatering calculations that the threshold of the $\mathrm{O} \mathrm{K}$ edge is dominated by the presence of the non-bridging oxygens in silicates [302], similarly to the case of borates. The multiple scattering calculations [277, 278], and later DFT calculations [279], have allowed reproducing the experimental data. Multiple scattering calculations on clusters of different sizes have allowed to reveal the contribution 
of the multiple scattering to the features due to medium range order. The effect of the core-hole has been discussed in these studies. While it is shown to be very important for the silicon $\mathrm{K}$ edge, [281] the contribution to the oxygen $\mathrm{K}$ edge has been described as small by $\mathrm{Wu}$ et al. [278], but it was shown to be non-negligible by Taillefumier et al. [279].

These authors highlighted that some cautions should be used to compare the calculated oxygen $\mathrm{K}$ edge and the $\mathrm{O} 2 \mathrm{p}$ pDOS, in reference to the work of Mo et al. [274] which presented strong disagreement between the oxygen $\mathrm{K}$ edge spectrum and pDOS calculations. While Mo et al. assigned this mismatch to energy dependence of the dipole matrix element, Taillefumier et al. have pointed out the influence of the calculation method on this energy dependence: the energy independence can be obtained with sufficiently small integration region used for the angular momentum projection [279].

Germanium oxides: The oxygen $\mathrm{K}$ edge of crystalline and amorphous germanates have been investigated to obtain structural information. Based on the study of crystalline references, oxygen $\mathrm{K}$ edge has been used to determine the coordination of $\mathrm{Ge}$ in amorphous systems. The similarity of the oxygen $\mathrm{K}$ edge of amorphous and crystalline (quartz) $\mathrm{GeO}_{2}$ confirmed the two-fold coordination of oxygen and the four-fold coordination of $\mathrm{Ge}$ in both cases as well as similar angles between the $\mathrm{GeO}_{4}$ tetrahedrons.

Five-fold $\mathrm{Ge}$ is observed in the alkali germanate glasses but only in compositions at the germanate anomaly maximum and beyond. It was confirmed that there is no formation of six-fold coordinated Ge within the glass before the germanate anomaly maximum [275].

No study of oxygen $\mathrm{K}$ edges in germanates have reported the contribution from non-bridging oxygens, even for high-alkali content glasses, although these are expected. Such interpretation is indeed subject to great caution because of the surface sensitivity of XAS, but here IXS could provide an alternative interesting probe. By comparison with the crystal references, Lelong et al. demonstrated with IXS measured oxygen $\mathrm{K}$ edge the formation of the five-fold coordinated $\mathrm{Ge}$ at the intermediate high pressure (5 GPa) from amorphous germania and the formation of 6-fold coordinated Ge at the higest pressure ( $18 \mathrm{GPa}$ ) [303]. McLeod et al. used oxygen $\mathrm{K}$ edge in light of calculations with Wienzk to study the oxygen vacancies in $\mathrm{GeO}_{x}$ nanoparticles as electrodes for lithium storage [304]. 


\subsubsection{Other p-element oxides}

Zinc oxides: $\mathrm{ZnO}$ is a common semiconductor oxide. The oxygen $\mathrm{K}$ edge of the hexagonal wurtzite polymorph has been measured by McLeod et al. [305]. Figure 38 shows the $\mathrm{O} \mathrm{K}$ edge spectrum of $\mathrm{ZnO}$. Because all $3 \mathrm{~d}$ orbitals are full, the spectrum reveals only the $\mathrm{O} 2 \mathrm{p}$ states hybridized with the $4 \mathrm{sp}$ states of the $\mathrm{Zn}$.

Guo et al. [306] compared the bulk spectrum with a thin film and nanorods. $\mathrm{ZnO}$ doped with transition metal ions (Fe, $\mathrm{Co}$ ) yields so-called dilute magnetic semiconductors. These systems have ferromagnetic order above room temperature and the combination of charge and spin degrees of freedom makes them useful for devices. Figure 38 shows that the dopping with Fe induce the presence of additional empty $3 \mathrm{~d}$ states at the onset of the $\mathrm{O} \mathrm{K}$ edge. Furthermore, the features observed for pure $\mathrm{ZnO}$ are broaden, probably due to the distortion of the crystal lattice. The oxygen $\mathrm{K}$ edge of $\mathrm{ZnO}$ doped with $\mathrm{Fe}$ and with Co mainly show the same spectral shape as pure $\mathrm{ZnO}$ with an additional peak due to the $\mathrm{Fe}-\mathrm{O}$ and $\mathrm{Co}-\mathrm{O}$ interaction [307309].

The oxygen $\mathrm{K}$ edges of the $[\mathrm{Cd}, \mathrm{Zn}] \mathrm{O}$ mixed oxides are discussed in the section on cadmium oxides.

Phosphates: Calcium phosphates are the main component of human bone. The materials include tricalcium phosphate $\mathrm{Ca}_{3}\left(\mathrm{PO}_{4}\right)_{2}$, and hydroxyapatite $\mathrm{Ca}_{5}\left(\mathrm{PO}_{4}\right)_{3} \mathrm{OH}$.

Rajendran et al. compare the calcium phosphate materials plus $\mathrm{CaCO}_{3}$ and $\mathrm{CaO}$ with the oxygen $\mathrm{K}$ edges from the bones of sheep, deer, chicken, etc. [311]. They use TEY and FY to differentiate between surface and bulk; they find that the surface of dried bone has a larger proportion of carbonate than the interior that is mainly made up of phosphates.

The phosphate systems with transition metal ions, $\left(\mathrm{LiFePO}_{4}, \mathrm{LiNiPO}_{4}\right.$, etc) are discussed in the sections of the respective transition metal ions.

Gallium oxides: The gallium oxide $\mathrm{Ga}_{2} \mathrm{O}_{3}$ exists in five polymorphs ( $\alpha$ to $\epsilon$ ). The $\beta-\mathrm{Ga}_{2} \mathrm{O}_{3}$ trigonal structure has recently got much attention in relation to possible optoelectronic as well as catalytic properties [312]. The oxygen $\mathrm{K}$ edge of $\beta-\mathrm{Ga}_{2} \mathrm{O}_{3}$ has been calculated with the FDMNES code by Hegde et al. [312]. Interestingly they do not find any significant difference between a calculation with and without core hole.

The oxygen $\mathrm{K}$ edge of $\beta-\mathrm{Ga}_{2} \mathrm{O}_{3}$ has also been published by Cocchi et al. [313] who find that their BSE calculation gives an accurate agreement with the experiment, in contrast to the calculation without core hole. 


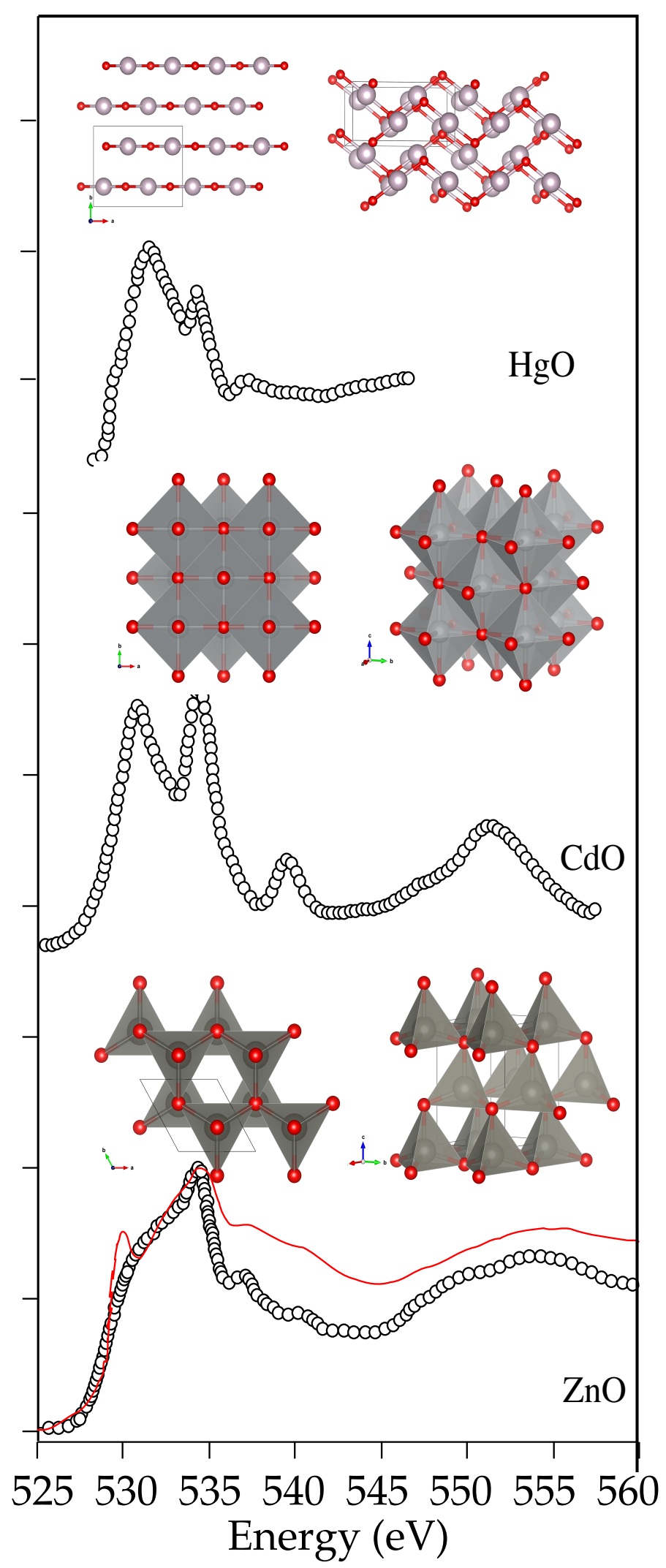

Figure 38: Oxygen $\mathrm{K}$ edge spectra of $\mathrm{ZnO}$ (wurtzite) compared to $\mathrm{Fe}: \mathrm{ZnO}$ (red) [307], $\mathrm{CdO}$ (rocksalt) [310], $\mathrm{HgO}$ (orthorombic, montroydite) [305]. Similarities in the spectrum shape and assignment are highlighted despite the differences in the crystal structures. 
Cadmium oxides: $\mathrm{CdO}$ is a rocksalt structured semiconductor. The oxygen $\mathrm{K}$ edge spectrum is measured by Demchenko et al. [314].

McLeod et al. compare the oxygen K edge with Wien2K calculations [305]. The bulk spectrum of $\mathrm{CdO}$ is compared with thin films that are measured both with electron yield and fluorescence yield. Detert et al. measured the oxygen $\mathrm{K}$ edges of the [Cd,Zn]O mixed oxides for different ratios of cadmium and zinc [310]. The phase transition between wurtzite $\mathrm{ZnO}$ and rocksalt $\mathrm{CdO}$ is visible in the oxygen $\mathrm{K}$ edge between $62 \%$ and $75 \% \mathrm{CdO}$. Figure 38 compares the spectra of $\mathrm{ZnO}$ and $\mathrm{CdO}$.

Indium oxides: Indium oxide $\mathrm{In}_{2} \mathrm{O}_{3}$ (cubic polymorph) is compared to the other $5 \mathrm{~s}^{0}$ oxides by McLeod et al. as reproduced in Figure 39.

The oxygen $\mathrm{K}$ edge of the cubic, rhombohedral, and orthorhombic $\mathrm{In}_{2} \mathrm{O}_{3}$ polymorphs have been measured by de Boer et al. [315] All polymorphs give similar $\mathrm{O} \mathrm{K}$ edge spectra with a first intense sharp peak assigned to the $\mathrm{O} 2 \mathrm{p}$ states hybridized to the cation empty $5 \mathrm{~s}$ states and then a variable second broad feature corresponding to the metal $5 \mathrm{p}$ states.

They calculated the spectra with Wien $2 \mathrm{~K}$ with and without core hole potential. The final state calculations accurately reproduce the observed spectral shapes.

a)

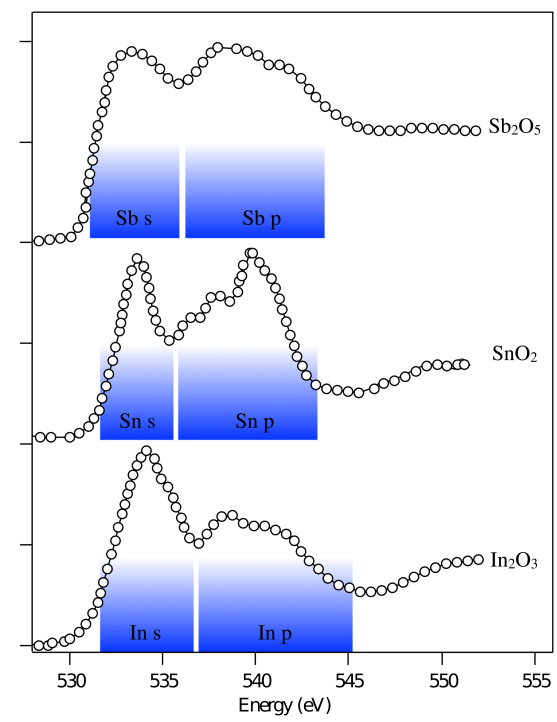

b)

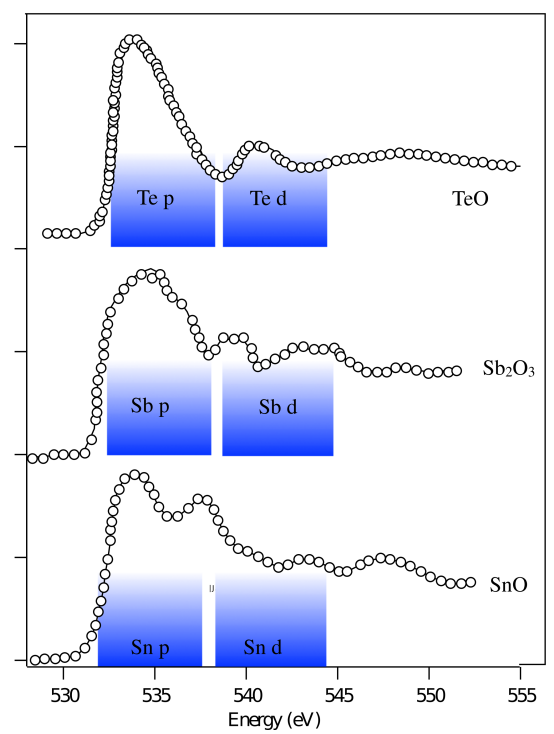

Figure 39: The oxygen $\mathrm{K}$ edge spectra of a) $5_{5}{ }^{0}$ oxides $\left(\operatorname{In}_{2} \mathrm{O}_{3}, \mathrm{SnO}_{2}, \mathrm{Sb}_{2} \mathrm{O}_{5}\right)$ and b) $5 \mathrm{~s}^{2}$ oxides $\left(\mathrm{SnO}, \mathrm{Sb}_{2} \mathrm{O}_{3}, \mathrm{TeO}_{2}\right)$. Data are reproduced from ref. [305] for all but $\mathrm{TeO}_{2}$ [316]. The cation contributions to the $\mathrm{O}$ 2sp anti-bonding states are given according to the p-DOS calculations from McLeod et al. [276] 
Tin oxides: Cassiterite $\mathrm{SnO}_{2}$ has a rutile structure and is the common ore for tin. The oxygen $\mathrm{K}$ edge is shown in Figure 39. It is shifted to lower energies but shows a similar shape than $\mathrm{GeO}_{2}$ and $\mathrm{SiO}_{2}$. This allows transposing the feature assignment from the Ge and $\mathrm{Si}$ well studied systems: the first intense peak corresponds to the $\mathrm{O} 2 \mathrm{p}$ hybridized with the $\sigma^{*}$ of the $\mathrm{Sn} 5 \mathrm{~s}$ states and the higher energy features correspond to the $\mathrm{Sn} \mathrm{p}$ and $\mathrm{pd}$ bands. The monoxide $\alpha$-SnO has a $\mathrm{PbO}$ structure. Upon heating, it converts to an intermediate tin-oxide $\mathrm{SnO}_{x}$, before converting to $\mathrm{SnO}_{2}$.

The EELS oxygen $\mathrm{K}$ edges of the three different oxides $\mathrm{SnO}, \mathrm{SnO}_{x}$ and $\mathrm{SnO}_{2}$ have been measured by Moreno et al. [317]. The oxygen $\mathrm{K}$ edge of the intermediate $\mathrm{SnO}_{x}$ phase has a shape that can be constructed from a linear combination of $\mathrm{SnO}$ and $\mathrm{SnO}_{2}$. Kurganskii measured the oxygen $\mathrm{K}$ edge of $\mathrm{SnO}_{2}$ and the calculated the spectrum with Wien2K [318]. A Wien2K calculation was performed with a core hole potential, but the agreement with experiment is not very good.

We note that the oxygen $\mathrm{K}$ edge of $\mathrm{SnO}_{2}$ is different as measured with EELS and with XAS. The EELS spectrum has two leading sharp peaks and the XAS spectrum only one. A possible explanation is that the EELS spectrum contains $\mathrm{SnO}$ that has its first peak at exactly the same energy.

Kronawitter et al. studied the interface between $\mathrm{SnO}_{2}$ and $\mathrm{TiO}_{2}$ [319]. Their $\mathrm{SnO}_{2}$ spectra are measured with TEY XAS and agree with the spectra from Kurganskii.

Antimony oxides: The oxygen $\mathrm{K}$ edge of antimony oxides, shown in Figure 39, were studied as part of a broader investigation of chemical bonding and hybridization in $5 p$ binary oxides by McLeod et al. [276] The authors further compare $5 \mathrm{~s}^{0}$ oxides $\left(\mathrm{In}_{2} \mathrm{O}_{3}, \mathrm{SnO}_{2}, \mathrm{Sb}_{2} \mathrm{O}_{5}\right)$ with $5 \mathrm{~s}^{2}$ oxides $(\mathrm{SnO}$, $\mathrm{Sb}_{2} \mathrm{O}_{3}, \mathrm{TeO}_{2}$ ).

Comparison of DOS and spectra calculations performed with Wien2k with experimental spectra reveals that in general the hybridization between $\mathrm{O} 2 \mathrm{p}$ states and cations states decreases with increasing cation atomic number. In the $5 \mathrm{~s}^{2}$ oxides the first relatively broad feature of the $\mathrm{O} \mathrm{K}$ edge is due to the antibonding $\mathrm{O} 2 \mathrm{p}$ bands mixed with the cation $5 \mathrm{p}$ while in the $5 \mathrm{~s}^{0}$ oxides, the cation $5 \mathrm{p}$ band is shifted to high energy and the first sharp feature is due to the empty cation $5 \mathrm{~s}$ band mixed with the $\mathrm{O} 2 \mathrm{p}$.

Tellurium oxides: $\mathrm{TeO}_{2}$ is an important building block for tellurite glasses used for nonlinear optics such as in optical fiber amplification. The oxygen $\mathrm{K}$ edge of $\mathrm{TeO}_{2}$ measured by Jiang et al. with EELS [316] is shown in Figure 39. The authors compare the spectrum with a number of different FEFF calculations. The core hole calculation gives equivalent results to the $Z+1$ 
calculation. To improve the correspondence with experiment a Z+2 approximation is used, but the agreement obtained is still not very good.

Mc Leod et al. have compared the $\mathrm{O}$ 2p DOS calculated with Wien $2 \mathrm{k}$ with the experimental spectra. The projected DOS reveal that the first peak in the $\mathrm{TeO}_{2} \mathrm{O} \mathrm{K}$ edge spectrum is dominated by $\mathrm{O} 2 \mathrm{p}$ anti-bonding states hybridized with Te $5 \mathrm{p}$ and the second peak is dominated by $\mathrm{O} 2 \mathrm{p}$ states hybridized with Te $4 \mathrm{~d}$.

Mercury oxides: Mercury the third element of the Group. Mercury oxide is a II-VI-type semiconductors. Divalent mercury ions have full $5 \mathrm{~d}$ orbitals and $6 \mathrm{~s}^{0}$ and $6 \mathrm{p}^{0}$ valence electronic configuration.

The oxygen $\mathrm{K}$ edge of mercury oxide $\mathrm{HgO}$, which structure consists of chains of $\mathrm{Hg}-\mathrm{O}$ in the montroydite form (orthorombic), has been compared with Wien2K calculations [305]. Figure 38 compares the spectral shape of $\mathrm{HgO}$ with $\mathrm{CdO}$ and $\mathrm{ZnO}$. As the cation increases in mass the core hole has a decreasing effect on the local conduction band probed by XAS measurements. There is close agreement between the measured XAS and the calculated DOS for $\mathrm{HgO}$.

Lead oxides: Qamar studied four different types of $\mathrm{PbO}$ samples: $\alpha-\mathrm{PbO}$ or litharge the red tetragonal polymorph, $\beta-\mathrm{PbO}$ or massicot, the yellow orhtorhombic polymorph, amorphous $\mathrm{PbO}$ and polycrystalline $\mathrm{PbO}$ [320]. They compare the experiments with Wien $2 \mathrm{~K}$ DFT calculations with and without the core hole potential. Oxygen $\mathrm{K}$ edges of lead oxides for leadacid batteries were published by Moseley et al. [321] The oxygen K edge of $\mathrm{BaPbO}_{3}$ has been measured in comparison with calculations with and without core hole [322].

The whole series of $\mathrm{Ba}(\mathrm{Pb}, \mathrm{Bi}) \mathrm{O}_{3}$ perovskites has been measured as a function of the bismuth doping. A gradual change from $\mathrm{BaPbO}_{3}$ to $\mathrm{BaBiO}_{3}$ is observed experimentally. Mastelaro et al. used the oxygen $\mathrm{K}$ edge to determine the hybridization between $\mathrm{Pb} 6 \mathrm{p}$ and $\mathrm{O} 2 \mathrm{p}$ orbitals in $\mathrm{Pb}_{1-\chi} \mathrm{La}_{\chi} \mathrm{TiO}_{3}$ compounds [323]. The $\mathrm{Pb} 6 \mathrm{p}$ peak appears in between the two Ti $3 \mathrm{~d}$ bands (see Ti oxides section). A similar approach with the support of FEFF calculations was used by to study the ferroelectric $\mathrm{Pb}_{1-\chi} \mathrm{Ba}_{\chi} \mathrm{Zr}_{0.65} \mathrm{Ti}_{0.35} \mathrm{O}_{3}$ compounds with changing the Ba loading [324]. Eberg et al. evidenced the decrease of the $\mathrm{Pb} 6 \mathrm{p}$ and $\mathrm{O} 2 \mathrm{p}$ and the $\mathrm{PbTiO}_{3} / \mathrm{SrTiO}_{3}$ interfaces with oxygen $\mathrm{K}$ edge and real-space multiple-scattering FEFF calculations [325].

Bismuth oxides: $\mathrm{Bi}_{2} \mathrm{O}_{3}$ exists in four crystallographic phases. Torruella et al. studied the monoclinic $\alpha-\mathrm{Bi}_{2} \mathrm{O}_{3}$ polymorph with EELS [326]. They compared the specral changes of a pellet with powder and nanowires and they studied the effect of defects on the spectral shape. $\mathrm{BaBiO}_{3}$ and the 


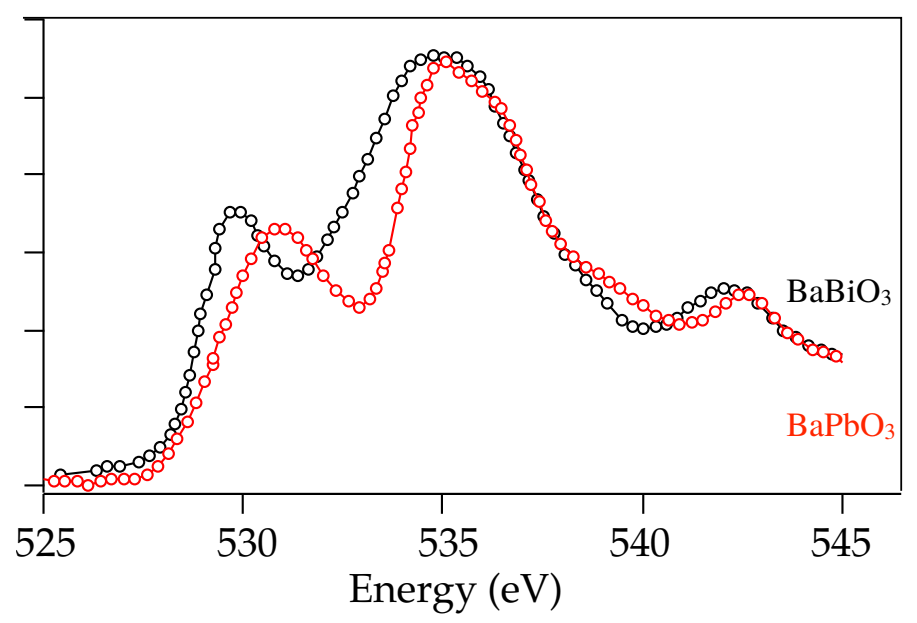

Figure 40: The oxygen $\mathrm{K}$ edge spectra of $\mathrm{BaBiO}_{3}$ and the $\mathrm{BaPbO}_{3} \cdot[322]$

$\mathrm{Ba}(\mathrm{Pb}, \mathrm{Bi}) \mathrm{O}_{3}$ perovskite systems have been discussed in the section on lead. $\mathrm{BiFeO}_{3}$ and $\mathrm{BiVO}_{4}$ are discussed in the sections on the transition metal ions. 


\section{ALKALINE EARTH METAL OXIDES}

Alkaline earth metals combined with oxygen, form oxides with a rocksalt structure and a well ordered surface.

These simple ionic solids have gained a lot of attention in the catalysis field thanks to the irreducibility and the stability of their surface that make them very efficient candidates for high temperature oxidative processes [327].

The basicity of the alkaline earth metal oxides surface has been extensively studied $[327,328]$ and the well established basicity trend follows the order: $\mathrm{MgO}<\mathrm{CaO}<\mathrm{SrO}<\mathrm{BaO}$ [329].

$\mathrm{CO}_{2}$ has been used as a probe for the surface reactivity of the $\mathrm{O}^{2-}$ site on the surface of $\mathrm{MgO}$ and $\mathrm{CaO}$ and the interaction energy decomposition led to the conclusion that $\mathrm{CaO}$ is a stronger base than $\mathrm{MgO}$ [330]. Among these oxides $\mathrm{MgO}$ is the one that obtained more attention because of the high surface area and the ease of preparation respect to $\mathrm{CaO}, \mathrm{SrO}$ and $\mathrm{BaO}$ where a thermal pretreatment of the sample is required [329].

By their chemical nature, the alkaline earth metal oxides should lie between the covalent III-V compounds and the alkali halides with ionic character.

The determination of the degree of covalency of the bond between the metal cation and the oxygen has a great importance as it affects the reactivity of the chemisorbed species [330, 331] and the sticking probabilities of small dipolar molecules [332] and metal atoms [333]. Despite their popularity, many controversy concerning the trend in ionicity through the series oxides are still open.

Two opposite views based on different argument exist: on the one hand, following the electronegativity values of the cations, Philips [334] and Van Veethem [335], the degree of covalency should decrease when going from $\mathrm{MgO}$ to $\mathrm{BaO}$, on the other hand, the bigger stabilization of $\mathrm{O}^{2-}$ ions in $\mathrm{MgO}$ and $\mathrm{BaO}$ due to a larger Madelung potential and the possible participation of empty $d$ orbitals into the covalent bond in heavier atoms seems to be suggest that the covalency increases along the period [336].

No simple way to measure the degree of covalency in ionic compounds is available, but a promising technique to measure the degree of covalent mixing has been the $\mathrm{x}$-ray absorption at the $\mathrm{O}(1 \mathrm{~s})$ edge.

It has already been shown [337] how it is possible to relate the XAS intensity 
distribution to the covalent character of the M-O bond for transition metal oxides. In fact in an ionic model the $2 p$ orbitals of the oxygen are full and the transition $1 s-2 p$ is not allowed. A certain degree of covalency makes states with $\mathrm{O} 2 p$ character available, so it is possible to relate also the intensity of the $\mathrm{O} 1 \mathrm{~s}$ excitation to the degree of covalency of the oxide [337].

Furthermore the oxygen $\mathrm{K}$ edge is one of the best method to obtain fingerprints of the electronic structure and atomic arrangement because the initial $1 \mathrm{~s}$ state is not degenerate and is strongly localized at the scattering center. The main advantage is that the dispersion is negligible and it causes no further crystal splittings [291].

The analysis of the oxygen $\mathrm{K}$ edge $\mathrm{x}$-ray absorption spectra of the alkaline earth metal oxides, taking into account the extent of the core hole effects and the trends of chemical shifts, combined with the study of the ground state properties of the electronic structure help to shed a lights on the nature of the $\mathrm{M}-\mathrm{O}$ bond.

\subsection{THEORY}

Since we are interested in the computation of $\mathrm{K}$ edges a reasonable approximation to the XAS spectra is given by density functional theory. The localized character of the core orbitals makes it possible to define a core excited Kohn-Sham states by constraining the occupation number of the 1s KohnSham orbital (see Chapter 1 ). The transition matrix elements are thus obtained within the single particle approximation by coupling the oxygen is electron of the ground state with the accessible empty states of the final state. The XCH method has been successfully applied to liquid and solid systems $[5,14]$, the underlying idea is that the core hole originating from the x-ray excitation is associate with the corresponding excited electron placed in the lowest unoccupied state. From Chapter 1 we know that in a single particle picture, the $\mathrm{x}$-ray absorption cross section can be written as:

$$
\sigma(\omega)=4 \pi^{2} \alpha_{0} \hbar \omega \sum_{f}\left|M_{i \rightarrow f}\right|^{2} \delta\left(E_{f}-E_{i}-\hbar \omega\right)
$$

where the photon energy and the fine structure constant are respectively $\hbar \omega$ and $\alpha_{0}$. The transition amplitude is written as:

$$
M_{i \rightarrow f}=\left\langle\Psi_{f}|D| \Psi_{i}\right\rangle
$$

$\mathrm{D}$ is the transition operator that can be expressed in the electric dipole or quadrupole approximation (see Chapter 1). Following the Blöch [338] procedure, the matrix element evaluation can be obtained within the projector 
augmented wave formalism [339]. We start transforming the all electron final wave function to the pseudowave function through the linear operator T as:

$$
\Psi_{f}=\mathrm{T}\left|\tilde{\Psi}_{f}\right\rangle
$$

The linear operator is written as:

$$
\mathrm{T}=1+\sum_{\mathrm{R}} \mathrm{T}_{\mathrm{R}}=1+\sum_{\mathrm{R}}\left(\left|\phi_{\mathrm{R}, \mathrm{n}}\right\rangle-\left|\tilde{\phi}_{\mathrm{R}, \mathrm{n}}\right\rangle\right)\left\langle\tilde{\mathrm{p}}_{\mathrm{R}, \mathrm{n}}\right|
$$

The sum is on $R$ where $R$ is the center of a spherical core region defined at the atomic site, also called augmentation region, the index $n$ is the angular momentum quantum number and $\left\langle\tilde{p}_{R n}\right|$ are projector augmented wave (PAW) projectors. $\phi_{R, n}$ and $\tilde{\phi}_{R, n}$ are the all electron and pseudo partial waves that coincide outside the augmentation region.

Combining Equation (2)(3) and (4) we obtain an expression for the transition amplitude as:

$$
M_{i \rightarrow f}=\left\langle\tilde{\psi}_{f}|D| \Psi_{i}\right\rangle+\sum_{R, n}\left\langle\tilde{\psi}_{f} \mid \tilde{p}_{R, n}\right\rangle\left\langle\phi_{R, n}|D| \psi_{i}\right\rangle-\sum_{R, n}\left\langle\tilde{\psi}_{f} \mid \tilde{p}_{R, n}\right\rangle\left\langle\tilde{\phi}_{R, n}|D| \psi_{i}\right\rangle
$$

The initial wave function is localized at the absorbing center $R_{0}$ so we can reduce the summation to only one term and we obtain for the initial state the expression:

$$
\left|\varphi_{R_{0}}\right\rangle=\sum_{n}\left|\tilde{p}_{R_{0, n}}\right\rangle\left\langle\phi_{R_{0}, n}|D| \psi_{i}\right\rangle
$$

So we can rewrite the expression for the absorption cross section as:

$$
\sigma(\omega)=4 \pi^{2} \alpha_{0} \hbar \omega \sum_{f}\left|\left\langle\tilde{\psi}_{f} \mid \tilde{\varphi}_{R_{0}}\right\rangle\right|^{2} \delta\left(E_{f}-E_{i}-\hbar \omega\right)
$$

Where the recursion method simplifies the computation of the spectra ensuring that only the occupied bands need to be calculated [339].

In order to quantify the amount of charge on each ion we performed a Bader analysis of the charge density [340] with VASP [341]. Within this formalism, the gradient paths of the electronic density terminating at an atom define a basin for this particular atom. Then by integrating over each basin, the charge which belongs to the corresponding atom is obtained.

In order to obtain information about the ground state bonding situation, Wannier functions have been computed for the oxygen $2 p$ states of the alkaline earth metal oxides series. Wannier functions can be interpreted as the solid state analog of the localized molecular orbitals for molecules [342]. 
Their construction starts from the Block functions by Fourier transformation. So we can write the Blöch function for an isolated band with index $n$ as:

$$
\Psi_{n, \mathbf{k}}(\mathbf{r})=e^{i \mathbf{k r}} \mathbf{u}_{n \mathbf{k}(\mathbf{r})}
$$

If we Fourier transform the vector $\mathbf{k}$ of the first Brillouin zone to the Bravais lattice vector $\mathbf{R}_{l}$ the Wannier function can be written as:

$$
w_{\mathrm{n}}\left(\mathbf{r}-\mathbf{R}_{l}\right)=\frac{\mathrm{V}}{(2 \pi)^{3}} \int_{\mathrm{BZ}} \mathrm{d} \mathbf{k} e^{-i \mathbf{k} \cdot \mathbf{R}_{l}} \Psi_{\mathrm{n}, \mathbf{k}}(\mathbf{r})
$$

The Fourier transformation is not unique, the freedom in choice of the Wannier function corresponds to the freedom in choice in the phases of Bloch orbitals as function of the wave vector $\mathbf{k}$ [342]. The orthogonality of the Wannier functions is ensured by the transformation properties of Blöch functions under lattice translations. The Wannier and hopping frequencies calculations have been performed with wien2k [343].

\subsubsection{Computational details}

All the DFT XAS calculations for the series $\mathrm{MgO}, \mathrm{CaO}, \mathrm{SrO}$, and $\mathrm{BaO}$ with a $\mathrm{NaCl}$ structure use PBE GGA functionals[344]. Ultrasoft pseudopotentials have been employed [345, 346]. The smoothness of the pseudowave functions is obtained by relaxing the norm conservation constraints and as a result less plane waves are necessary [345]. The kinetic energy cutoff for electronic wave functions and the density is respectively 30 and $240 \mathrm{Ry}$. As periodic boundary conditions are used, the core hole interaction is taken into account in a supercell approach that allows the isolation of the absorbing atom.

The supercell size employed for $\mathrm{MgO}, \mathrm{CaO}, \mathrm{SrO}$ and $\mathrm{BaO}$ is a $2 \times 2 \times 2$, while

Table 18: Crystallographic structure of alkaline earth metal oxides used in XAS calculations from references: a) [347], b) [348], c) [349] and d) [350].

\begin{tabular}{lllll}
\hline & $\mathrm{MgO}^{\mathrm{a}}$ & $\mathrm{CaO}^{\mathrm{b}}$ & $\mathrm{SrO}^{\mathrm{c}}$ & $\mathrm{BaO}^{\mathrm{d}}$ \\
\hline $\mathrm{a}=\mathrm{b}=\mathrm{c}(\AA)$ & 4.216 & $4.8 \mathrm{o} 5$ & $5 \cdot 5.160$ & $5 \cdot 523$ \\
$\alpha=\beta=\gamma$ & $90^{\circ}$ & $90^{\circ}$ & $90^{\circ}$ & $90^{\circ}$ \\
Cryst. syst. & Cubic & Cubic & Cubic & Cubic \\
Space group & $F m-3 m$ & $F m-3 m$ & $F m-3 m$ & $F m-3 m$ \\
\hline
\end{tabular}


Table 19: Crystallographic structure of carbonates and hydroxides used in XAS calculations from references: a) [351], b) [352], c) [353], d) [354] e) [355] and f) $[356]$.

\begin{tabular}{|c|c|c|c|c|c|c|}
\hline & $\mathrm{CaCO}_{3}{ }^{\mathrm{a}}$ & $\mathrm{Ca}(\mathrm{OH})_{2} \mathrm{~b}$ & $\mathrm{SrCO}_{3}{ }^{\mathrm{c}}$ & $\mathrm{Sr}(\mathrm{OH})_{2}{ }^{\mathrm{d}}$ & $\mathrm{BaCO}_{3}{ }^{\mathrm{e}}$ & $\mathrm{Ba}(\mathrm{OH})_{2}{ }^{\mathrm{f}}$ \\
\hline & $a=b=c 6.360$ & $a=b 3.586$ & a 5.090 & $a=b 8.990$ & a 5.280 & a 11.0818 \\
\hline Cell Param. & & c 4.880 & b 8.358 & c 11.566 & b 8.830 & b 16.602 \\
\hline Cell Angle. & $\alpha=\beta=\gamma 46^{\circ}$ & $\begin{array}{c}\alpha=\beta 90^{\circ} \\
\gamma 120^{\circ}\end{array}$ & $\begin{array}{c}\text { C } 5.997 \\
\alpha=\beta=\gamma 90^{\circ}\end{array}$ & $\alpha=\beta=\gamma 90^{\circ}$ & $\begin{array}{c}\text { c } 6.390 \\
\alpha=\beta=\gamma 90^{\circ}\end{array}$ & $\begin{array}{c}\text { c } 7.125 \\
\alpha=\beta=\gamma 90^{\circ}\end{array}$ \\
\hline $\begin{array}{l}\text { Cryst. syst. } \\
\text { Space Group }\end{array}$ & $\begin{array}{c}\text { Trigonal } \\
R-3 c\end{array}$ & $\begin{array}{c}\text { Trigonal } \\
P-3 m 1\end{array}$ & $\begin{array}{l}\text { Orthorhombic } \\
\text { Pmcn }\end{array}$ & $\begin{array}{c}\text { Tetragonal } \\
P_{4} / n c c 1\end{array}$ & $\begin{array}{c}\text { Orthorhombic } \\
\text { Pmcn }\end{array}$ & $\begin{array}{l}\text { Orthorhombic } \\
\text { Pnma }\end{array}$ \\
\hline
\end{tabular}

for the carbonates and the hydroxides, given the big dimension of the system a IXIXI cell has been employed.

The ground state calculations of the partial density of states (PDOS) have been performed in order to obtain a representation of the occupied and unoccupied states by means of Löwdin charge population analysis.

All the XAS calculations have been performed with the Quantum Espresso QE suite[357]. Calculations were carried out from the experimental crystal structures listed in Table 18 and 19. In order to perform the k-point sampling and generate the unoccupied states for the core excited spectra, the Shirley interpolation scheme has been adopted[358]. The transition amplitudes have been obtained within the PAW frozen-core approximation[339]. The spectra have been aligned with the experimental results using a rigid shift of $525.65 \mathrm{eV}$.

Wannier intepolation has been performed on a 40x40x40 mesh in real space. The Wannier functions and hopping frequencies calculations have been performed with Wien2k[343].

\subsection{ELECTRONIC STRUCTURE}

\subsubsection{Partial density of states (PDOS)}

Alkaline earth metal oxides share the same rocksalt structure. The increase in lattice parameter along the series is related to the increase in the cation size (see Table 18). In case of ionic solid the electron localization is high, ensuring little dispersion in the band structure where each band mainly maintain the character of the atomic orbital.

It is well known that band gap, as transport properties, obtained within 

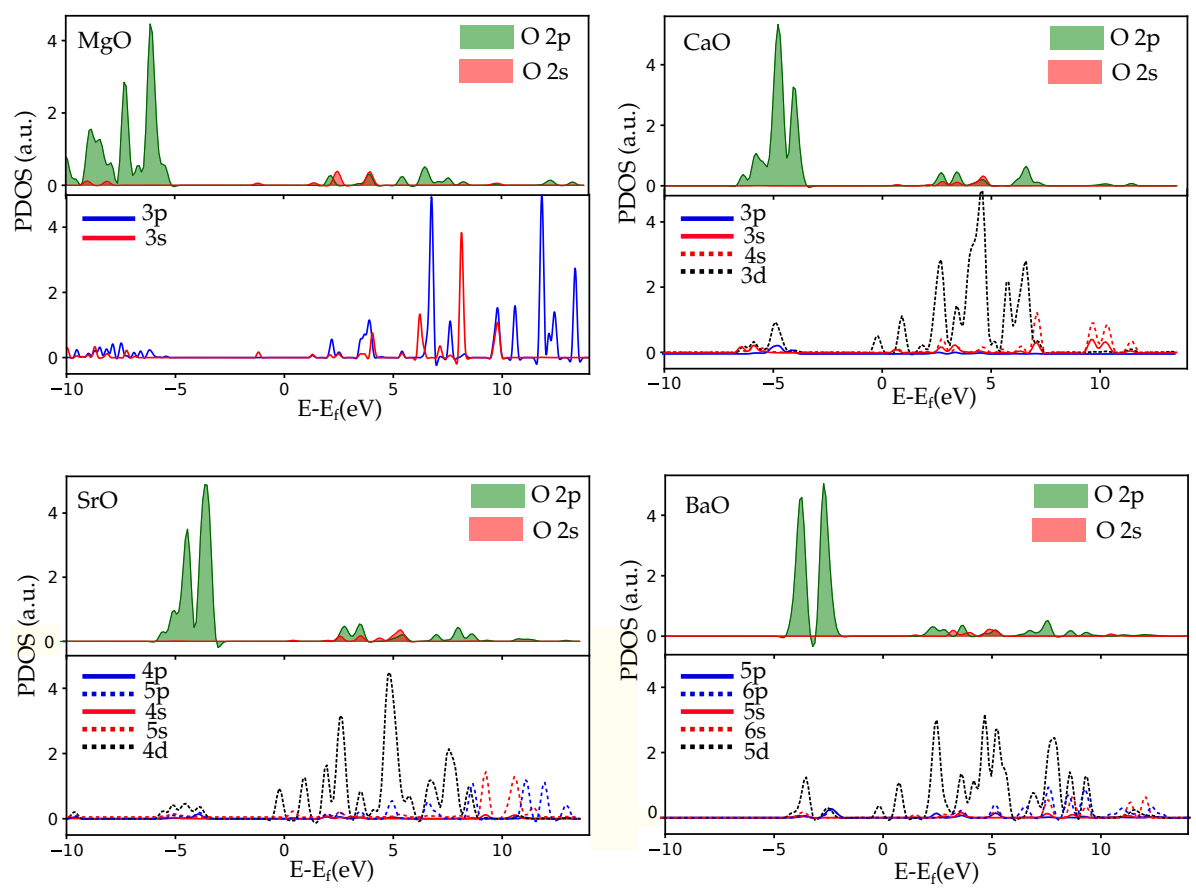

Figure 41: Partial density of states (s,p and d PDOS) for the oxygen(upper panels) and the metal(lower panel). The Fermi energy is taken as 0.

standard DFT calculations are not very well reproduced and it is indeed not surprising the obtained value $6.03 \mathrm{eV}(\mathrm{MgO}), 5.0 \mathrm{eV}(\mathrm{CaO}), 5.04 \mathrm{eV}(\mathrm{SrO})$ and $3.83 \mathrm{eV}(\mathrm{BaO})$ turns to be underestimated respect to the experimental results $((7.8 \mathrm{eV}, 7.1 \mathrm{eV}, 5.9 \mathrm{eV}$, and $4.3 \mathrm{eV}$ respectively [359]), anyway the trend is still reproduced.

Alkaline earth metal oxides show a high degree of ionicity and the outermost valence band turns to be constitute by mainly states originating from the oxygen $2 p$ orbitals. Figure 41 shows the oxygen and metal PDOS. In the plane wave pseudopotential framework, the PDOS is obtained by projecting the pseudowavefunction onto the pseudoatomic partial waves with required symmetry. Going from $\mathrm{MgO}$ to $\mathrm{BaO}$ a narrowing of the oxygen 2p band is reported (Figure 41: $4.6 \mathrm{eV} \mathrm{MgO,} 2.36 \mathrm{eV} \mathrm{CaO,} 1.91 \mathrm{eV} \mathrm{SrO}$ and $1.06 \mathrm{eV} \mathrm{BaO}$ ). The band widths reported are overestimated (in accordance with earlier DFT [360] and Hartree-Fock calculations [361]), respect to the experimental result obtained with electron momentum spectroscopy (EMS)[361], but the clear decreasing trend is still reproduced. The extent of band dispersion is usually linked to the degree of delocalization. Skorodumova et al. [360] linked the shrinking of the oxygen $2 \mathrm{p}$-valence states to a repulsive interaction between the semicore p-metal states and the oxygen $\mathrm{p}$ states by examining the progressive shift toward the top of the valence band of the metal semicore p-states (around 15-20 eV below the Fermi energy not 


\begin{tabular}{llll}
\hline $\mathrm{MgO}$ & $\mathrm{CaO}$ & $\mathrm{SrO}$ & $\mathrm{BaO}$ \\
\hline $\mathrm{Mg}+2$ & $\mathrm{Ca}+1.42$ & $\mathrm{Sr}+1.41$ & $\mathrm{Ba}+1.45$ \\
\hline
\end{tabular}

Table 20: Atomic charge by Bader charge partitioning.

shown in Figure 41). However we can explain the narrowing of the oxygen p-states on the basis of the crystal structure without invoking changes in the metal-oxygen interaction, in fact the distance oxygen-oxygen increases along with the lattice parameter causing a decrease in the repulsion energy and in orbital overlap between the anions. So the decrease in band width is the consequence of a reduction in separation between the bonding and antibonding states within the same oxygen band. Furthermore the Bader charge partitioning results in Table 20 suggests that an unweighed interpretation of the differences in the valence band dispersion in terms of a different extent of localization of the metal-oxygen bond would lead to wrong conclusions. The Bader analysis indeed indicates that all the oxides have an ionic character, with the $\mathrm{MgO}$ being the most ionic in the series. If we turn our attention to the metal states above the Fermi energy we observe a different character of the unoccupied states where the d-states contribution for $\mathrm{Ca}, \mathrm{Sr}$ and $\mathrm{Ba}$ becomes predominant at the bottom of the conduction band. In $\mathrm{MgO}$ the d-states are not included in the calculation since it has been already proven that they are too high in energy to participate in the metal-oxygen bonding interaction [336]. The following examination of the Wannier functions for the alkaline earth metal oxides series reveals important differences in terms of metal-oxygen bond localization.

\subsubsection{Wannier functions}

In order to investigate the nature of the metal-oxygen bond character we analyse the Wannier functions for the bonding state due to metal and oxygen $2 p$ hybridization. Wannier functions are uniquely defined for insulators and semiconductors and provides a description of the hybridization and spatial distribution of the charge density [342].

Figure 42 shows the band structure and the related Wannier functions for the oxygen $2 p$ states, where the spread of the Wannier functions is reported in the caption. The spatial distribution of the Wannier functions increases along the $(\mathrm{a}, \mathrm{O}, \mathrm{o})$ direction going down through the alkaline earth metal oxides series. The reported oxygen $2 p$ spread increases from $0.904 \AA$ to $1.638 \AA$ 

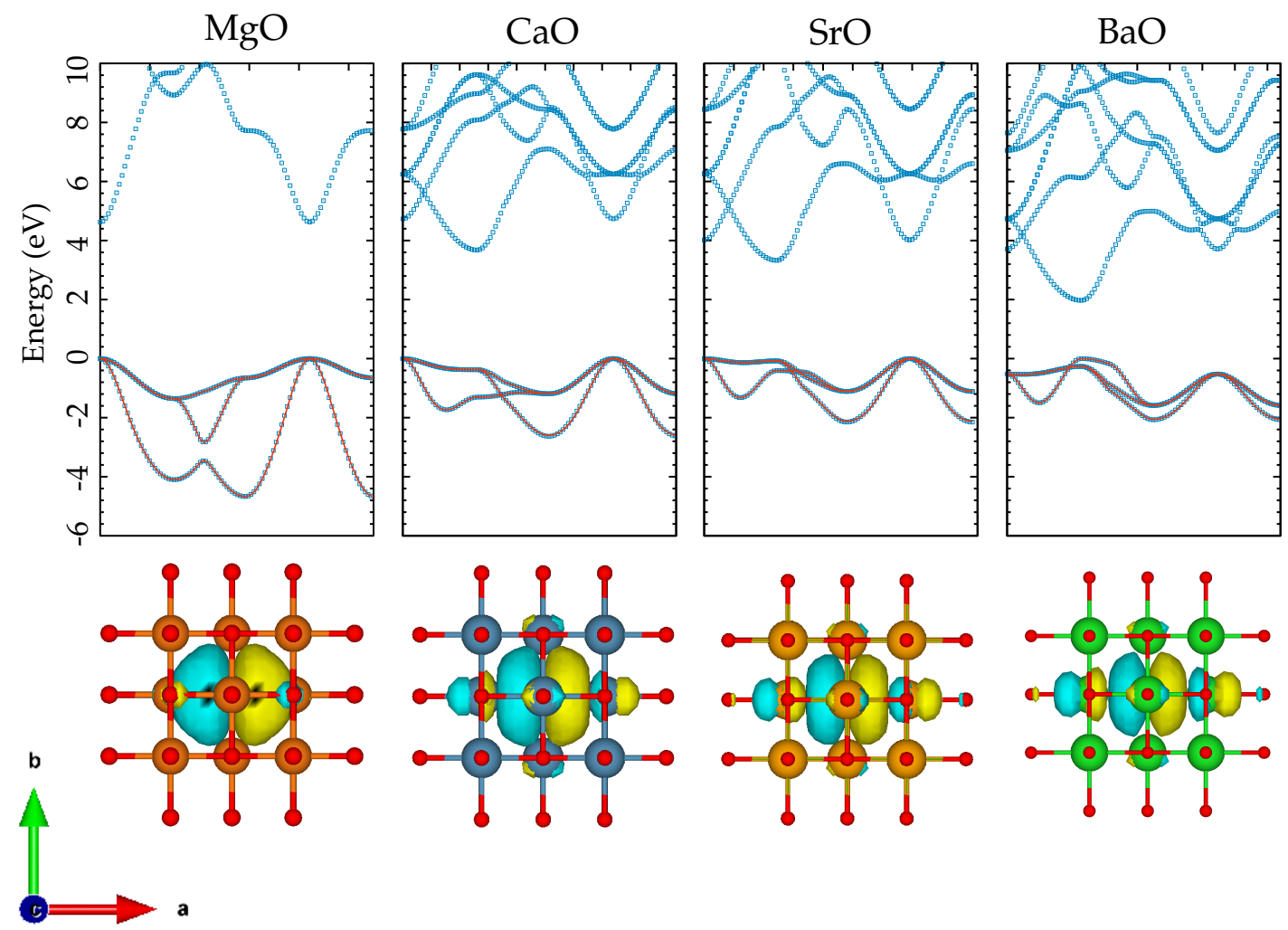

Figure 42: Wannier functions obtained for the oxygen $2 p$ states and the associated band structure. Oxygen atoms are in red. The Wannier function spread is $0.904 \AA$ for $\mathrm{MgO}, 1.147 \AA$ for $\mathrm{CaO}, 1.310 \AA$ for $\mathrm{SrO}$ and $1.638 \AA$ for $\mathrm{BaO}$. The isosurface value is set to 0.001 for all the plots. 


\begin{tabular}{lllll}
\hline & $\mathrm{MgO}$ & $\mathrm{CaO}$ & $\mathrm{SrO}$ & $\mathrm{BaO}$ \\
\hline $\mathrm{t}_{1}$ & 0.2626 & 0.0791 & 0.0210 & -0.0403 \\
$\mathrm{t}_{2}$ & -0.3486 & -0.1260 & -0.0886 & -0.0342 \\
$\mathrm{t}_{3}$ & 0.0603 & 0.2230 & 0.2641 & 0.3090
\end{tabular}

Table 21: Two centers hopping integrals $(\mathrm{eV})$ for the systems $\mathrm{MgO}, \mathrm{CaO}, \mathrm{SrO}$ and $\mathrm{BaO}$. Where the combination of $t_{1}$ and $t_{2}$ gives respectively the ppo $\left(-t_{1}-t_{2}\right)$ and $\mathrm{pp} \pi\left(-\mathrm{t}_{1}+\mathrm{t}_{2}\right)$ Slater-Koster integrals.

going from $\mathrm{MgO}$ to $\mathrm{BaO}$ reinforcing the hypothesis that the localization is higher in the $\mathrm{MgO}$ system. The spread and the shape of the Wannier functions can be rationalized in terms of hopping integrals.

Hopping integrals couple different states and allow the electron hopping from one atom to one of its neighbors, they are the off-diagonal matrix elements that can be extracted from the interaction Hamiltonian. For the alkaline earth metal oxides, we consider the 3 available hopping channels between nearest neighbour oxygen atoms, denoted as $t_{1}, t_{2}$ and $t_{3}$ and defined in Figure 43. From table 21, we see that the $t_{3}$ channel become deter-
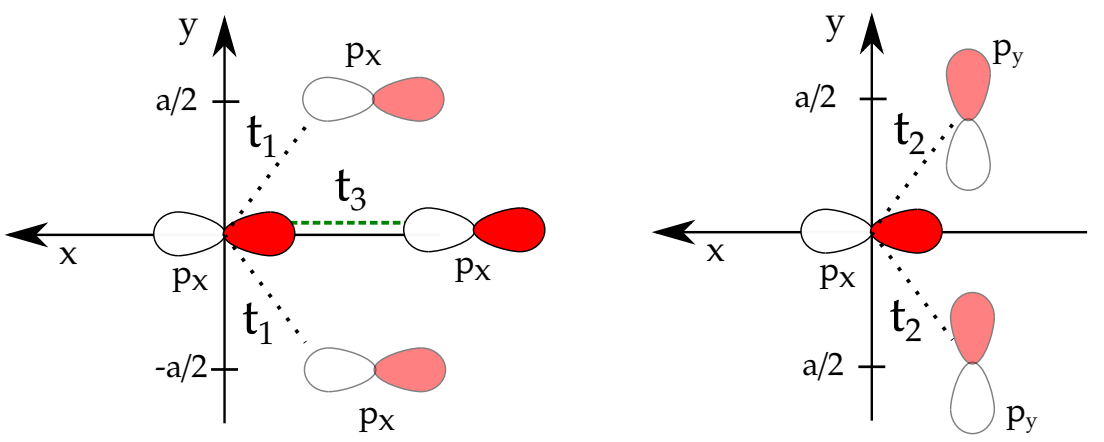

Figure 43: Pictorial representation of the three hopping integrals between nearest neighbour oxygen atoms.

minant in the hopping process going from $\mathrm{MgO}$ to $\mathrm{BaO}$. This behavior is clearly reflected in the shape of the px-like Wannier functions for $\mathrm{SrO}$ and $\mathrm{BaO}$, showing a large spread along $(\mathrm{a}, \mathrm{O}, \mathrm{O})$ direction. From the band structure in Figure 42 we can rationalize this behavior, in fact, going from $\mathrm{MgO}$ to $\mathrm{BaO}$, the metal bands above Fermi level come down in energy and start hybridizing with the oxygen $2 p$ orbitals. 


\subsection{1 $\mathrm{MgO}$}

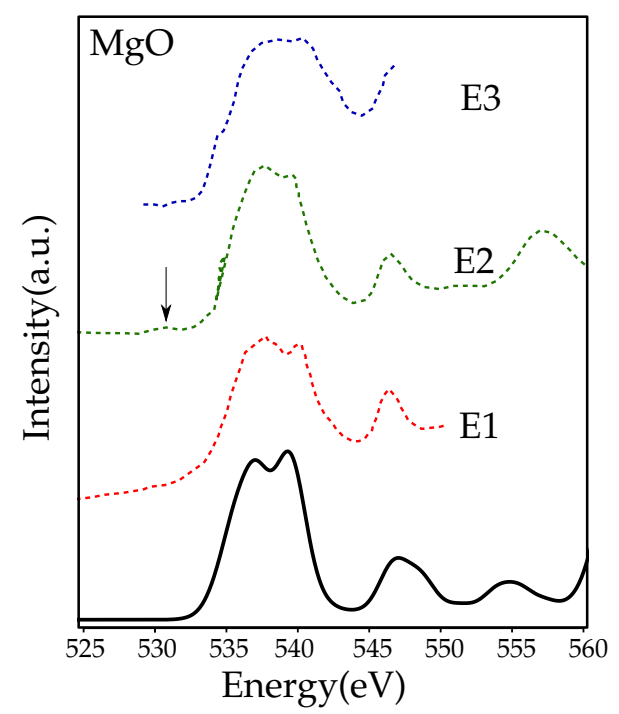

Figure 44: Right panel: Calculated oxygen $\mathrm{K}$ edge spectra of $\mathrm{MgO}$ black line, compared with the experimental results from E3 [305],E2 [362] and E1 [363]. A gaussian broadening of $0.8 \mathrm{eV}$ as been applied to the calculated spectra and the experimental data have been shifted for the comparison.

Figure 44 shows the calculated oxygen $\mathrm{K}$ edge spectra of $\mathrm{MgO}$ compared with experimental spectra from $\operatorname{ref}[305,362,363]$. The calculated and the experimental spectra of $\mathrm{MgO}$ matches well and show four main regions around 536, 540, 545 and $551 \mathrm{eV}$. In the $\mathrm{MgO}$ experimental spectra from [362] a small peak before the near edge structure is observed around $530 \mathrm{eV}$; the peak has been ascribed to the presence of a core exciton. In the theoretical work of Pascual [364], the origin of this peak has been attributed to a surface effect corresponding to the $1 \mathrm{~s} \longrightarrow 3 \mathrm{p}_{z}$, transition polarized out from the surface. The Fermi energy obtained for the $\mathrm{MgO}$ system in our calculations is $11.3264 \mathrm{eV}$ and only the $\mathrm{Mg}$ 3s partial density of state (PDOS) reveals the presence of s-character states at $10.15 \mathrm{eV}$. The presence of peak at 530 $\mathrm{eV}$ suggests that in order to obtain a complete theoretical description of the oxygen $\mathrm{K}$ edge spectra, simulations that take into accounts surface effects are required. 


\section{$5 \cdot 3 \cdot 2 \mathrm{CaO}$}

Figure 45 shows the calculated $\mathrm{CaO}$ oxygen K-edge spectra compared with a set of experimental results from ref. [305, 363, 365, 366]: some discrepancies with the experimental data from $\mathrm{E}_{1}$ and $\mathrm{E}_{3}[305,363]$ can be easily spotted. The intensity of the higher energy shoulder of the spectra does not

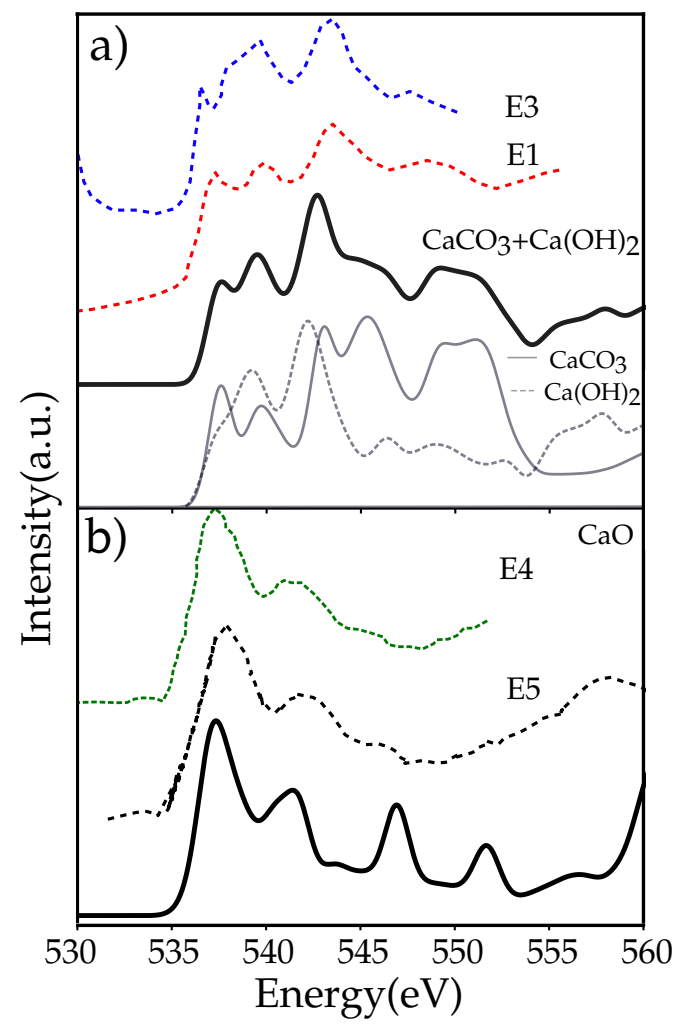

Figure 45: a)Calculated oxygen $\mathrm{K}$ edge spectra of $\mathrm{CaO}$ (black line), compared with the experimental results from: $\mathrm{E}_{4}$ [365], $\mathrm{E}_{5}$ [366]. b)Calculated oxygen $\mathrm{K}$ edge spectra of $\mathrm{Ca}(\mathrm{OH})_{2}+\mathrm{CaCO}_{3}$ (black line), $\mathrm{Ca}(\mathrm{OH})_{2}$ (grey dotted line) and $\mathrm{CaCO}_{3}$ (grey line) compared with the experimental results from: E3 [305], E1 [363].A gaussian broadening of $0.8 \mathrm{eV}$ as been applied to the calculated spectra and the experimental data have been shifted for the comparison.

matches with the calculation and the second peak is not well defined.

It is well known that the surface of $\mathrm{CaO}$ is susceptible to water adsorption leading to formation of $\mathrm{Ca}(\mathrm{OH})_{2}$ via an exothermic reaction [367] because of the higher basicity of the surface respect of $\mathrm{MgO}$. Furthermore commercially available $\mathrm{CaO}$ is usually prepared by thermal decomposition of $\mathrm{Ca}(\mathrm{OH})_{2}$ and the contamination can be also arise from a $\mathrm{H}_{2} \mathrm{O}$ synthesis residual. Also the carbonate contamination cannot be ignored since $\mathrm{CaO}$ has a good affinity for $\mathrm{CO}_{2}$ especially at high temperature [368]. The lack of details on how 
the materials were handled prior to the measurement suggests the main reason for the mismatch is linked to contamination from water and $\mathrm{CO}_{2}$. In order to find the reason of the mismatch, the spectra of $\mathrm{Ca}(\mathrm{OH})_{2}$ and $\mathrm{CaCO}_{3}$ have been computed. A good agreement with the experimental spectra from $\mathrm{E}_{1}$ and $\mathrm{E}_{3}[305,363]$ can be found if the spectra for $\mathrm{Ca}(\mathrm{OH})_{2}$ and $\mathrm{CaCO}_{3}$ are summed up and averaged.

The experimental EELS results $\mathrm{E}_{5}$ [366] and the EELS $\mathrm{E}_{4}$ [365] matches very well with the calculated oxygen $\mathrm{K}$ edge $\mathrm{CaO}$ spectra.

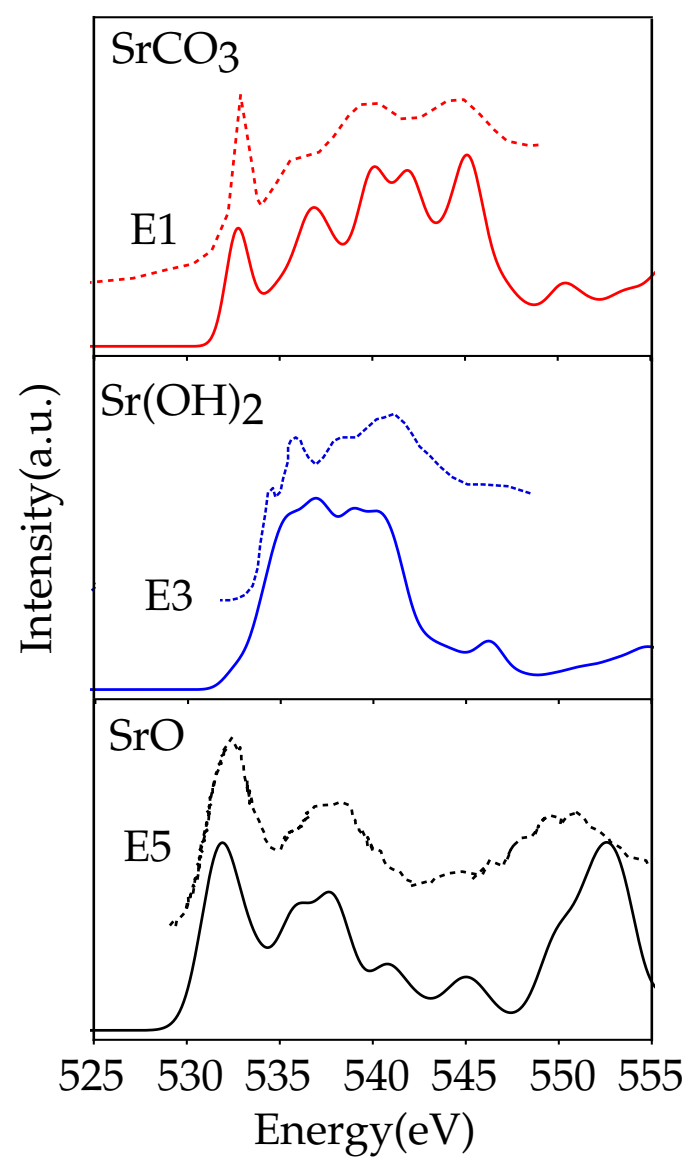

Figure 46: Calculated oxygen $\mathrm{K}$ edge spectra of SrO black line, $\mathrm{Sr}(\mathrm{OH})_{2}$ blue line and $\mathrm{SrCO}_{3}$ red line, compared with the experimental results from references: $\mathrm{E}_{1}$ [363], E3 [305], E5 [366]. A gaussian broadening of o.8eV as been applied to the calculated spectra and the experimental data have been shifted for the comparison.

\section{$5 \cdot 3 \cdot 3 \mathrm{SrO}$}

In Figure 46 a comparison between the experimental and the calculated spectra of $\mathrm{SrO}$ is reported. A good agreement is found between the experimental spectra $E_{5}$ [366] and the calculated spectra, while the experimental results 
E1 and $E_{3}[305,363]$ show a pronounced first peak [363] and a high energy bump missing in the calculated one.

In order to investigate the origin of the mismatch, the spectra of $\mathrm{SrCO}_{3}$ and $\mathrm{Sr}(\mathrm{OH})_{2}$ have been computed. In Figure 46 the experimental spectra for $\mathrm{SrO}$ are compared with the calculated spectra of $\mathrm{SrCO}_{3}$ and $\mathrm{Sr}(\mathrm{OH})_{2}$. In these cases we can see a match between the experiment and the theoretical data. Commercially available $\mathrm{SrO}$ is prepared from $\mathrm{SrCO}_{3}$ and if a commercial $\mathrm{SrO}$ has been exposed to the atmosphere its surfaces may be covered with carbonates, hydroxides, and in some cases, peroxides [329]. Removal of carbon dioxide, water and oxygen from the surfaces is required to reveal the oxide surfaces. So also in this case the reason of the mismatch has been linked to a carbonate and hydroxide contamination.

\section{$5 \cdot 3 \cdot 4 \quad \mathrm{BaO}$}

In Figure 47 the oxygen $\mathrm{K}$ edge spectra for $\mathrm{BaO}$ is compared with the experimental results E1,E3 and E6 [289, 305, 363]. The comparison with all the experimental data is rather poor.

Figure 47 shows also the spectra for $\mathrm{Ba}(\mathrm{OH})_{2}$ and $\mathrm{BaCO}_{3}$ compared with the experimental data: the shape of the carbonate and the hydroxide spectra present a good agreement with the experimental results E1 and E3 [305, 363]. The experimental spectra from ref. [289] has been collected is a controlled atmosphere in order to prevent any possible $\mathrm{H}_{2} \mathrm{O}$ and $\mathrm{CO}_{2}$ contamination from the chamber and possible chemical reduction reactions.

The agreement with the calculated and the measured [285] spectra is poor, the first peak is not sharp and too much emphasis appears in the second peak. We do not try to improve here the calculations for $\mathrm{BaO}$ since we want to use a consistent method for all the computed XAS but anyway even in this case a possible contamination cannot be excluded.

The good agreement of the calculations with the some of the available experimental data confirms the good performance of the KS-DFT in reproducing $\mathrm{K}$ edges spectra. A comparison of the spectra obtained for the alkaline earth metal oxides series reveals significant differences in the near edge structure in terms of spectral features and energy positions. The shape of the first peak changes going from $\mathrm{MgO}$ to $\mathrm{BaO}$ and the energy position of the first peak decreases from $\mathrm{MgO}$ to $\mathrm{BaO}$. 


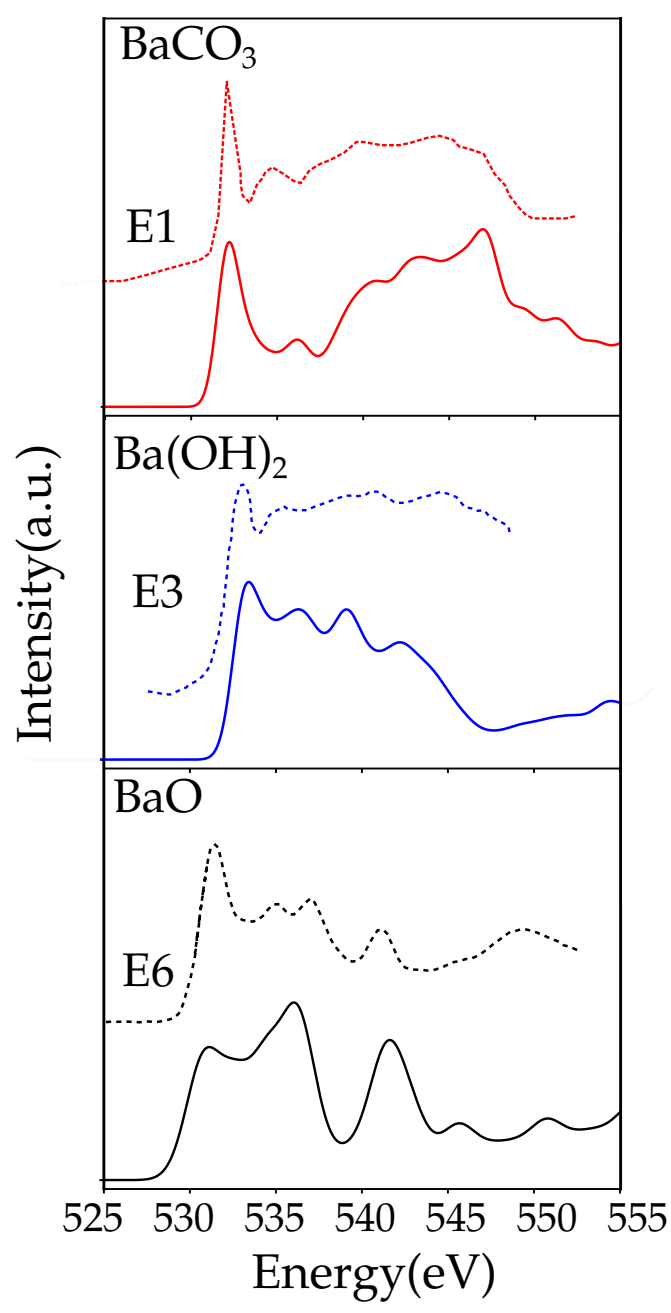

Figure 47: Calculated oxygen $\mathrm{K}$ edge spectra of $\mathrm{BaO}$ black line, $\mathrm{Ba}(\mathrm{OH})_{2}$ blue line and $\mathrm{BaCO}_{3}$ red line, compared with the experimental results from references E1 [363], E2 [305], E6 [289]. A gaussian broadening of $0.8 \mathrm{eV}$ as been applied to the calculated spectra and the experimental data have been shifted for the comparison. 


\subsection{CORE HOLE EFFECTS}

In Figure 48, all the calculated XCH spectra for the alkaline earth metal oxides series are reported compared with the spectra without the inclusion of the core hole treatment.

An estimation of the core hole effects in the oxygen $\mathrm{K}$ edge spectra of the alkaline earth metal oxides provides a useful tool for the interpretation of the spectra and shows the importance of the core hole treatment for a correct description of the experimental spectra.

For all the spectra in Figure 48 different extents of core hole influence can be evinced. The core hole treatment affects more the low energy spectral features because of the localized character of the final electronic states. In the oxygen $\mathrm{K}$ edge spectra of $\mathrm{MgO}$, the omission of the core hole treatment leads to a strong underestimation of the low energy peak.

The inclusion of core hole potential has the effect of drawing unoccupied
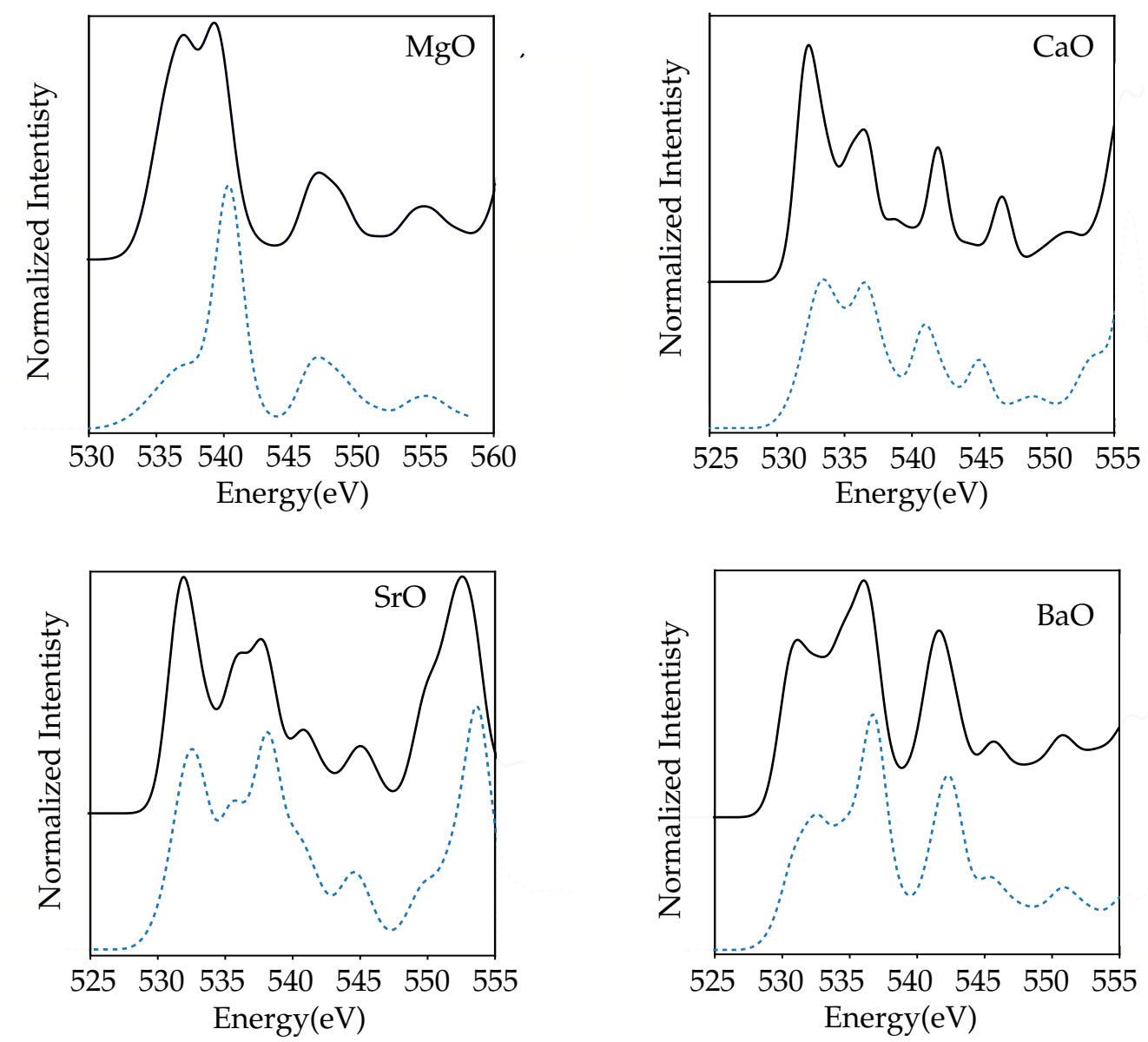

Figure 48: Calculated oxygen $\mathrm{K}$ edge $\mathrm{XCH}$ spectra of $\mathrm{MgO}, \mathrm{CaO}, \mathrm{SrO}$ and $\mathrm{BaO}$ (black line) compared with the no core hole results (blue dotted line).The applied gaussian broadening is $0.8 \mathrm{eV}$. 
electronic states toward the threshold energy, contracting the wave function and increasing the weight of the spectral features near the absorption edge [30]. The spectra without the core hole treatment reported for $\mathrm{MgO}$ shows a good match with the oxygen 2p PDOS Full-potential Linearized Augmented Plane Wave method (FLAPW) in reference [369] suggesting a good equivalency between the ground state PDOS and the no-core-hole spectra.

In the $\mathrm{CaO}$ case, the alteration induced by the absence of the core hole treatment are similar to the $\mathrm{MgO}$ case but less dramatic, in this case the intensity of the first peak is underestimated and the peak position are fairly reproduced. The core hole effects for the oxygen $\mathrm{K}$ edge spectra of $\mathrm{SrO}$ and $\mathrm{BaO}$ are rather similar, in this case the omission of the core-hole treatment does alter less the near edge spectral features compared to the $\mathrm{MgO}$ and $\mathrm{CaO}$ case. As general trend the first feature gains intensity with the inclusion of the core hole.

The extent of the core hole effects can give information about the electron localization [370] and the screening of the valence electrons [30]. Stronger distortions are introduced in $\mathrm{K}$ edges spectra when the core hole treatment is omitted respect to $L$ edges of metals and this behavior is linked to a different degree of electronic distribution localization of $\mathrm{p}$-valence and s-valence states [371].

Within the oxygen $\mathrm{K}$ edges spectra obtain for the alkaline earth metal oxides, the explicit treatment of the core hole potential becomes less influential for the heavier elements of the series suggesting that the electron localization is stronger for the lighter $\mathrm{MgO}$ and $\mathrm{CaO}$ (where the core hole effects are more evident) respect to $\mathrm{SrO}$ and $\mathrm{BaO}$. A consequence of the localization is the decrease in the orbitals overlap that leads to a reduction in the degree of covalency of the M-O bond [370]. The results from the Bader analysis in table 20 support the trend obtained by the analysis of the core hole effects.

\section{$5 \cdot 5$ CHEMICAL SHIFTS}

Another methodology widely adopted [10, 372-374] to obtain information about the ionicity of the materials is based on the inspection of the binding energy.

Binding energies are not just fingerprints of the absorbing atom but contain information about the bonding situation in which the absorbing atom is involved. Binding energies are usually obtained by X-ray photoelectron 
spectroscopy [375-377] and they can be further used to obtain the chemical shift between different systems $[378,379]$. The ionic character of metal oxides has been correlated with the measured oxygen is core level binding energy [380]. In the oxides case, the initial state charge displacement originating from the different electronegativities of the anions and the cations decreases the electrostatic potential felt by the core electrons of the oxygen leading to a decrease in the anion core binding energy depending on the anion charge (from Tab. 20 the decrease goes down through the series ).

Defining the chemical shift for a given atom in different systems reveals information on the chemical surroundings of the probe. In order to obtain the binding energy chemical shifts for the alkaline earth metal oxides series, we look for the energy of the initial ground state without the core hole $(\mathrm{NCH})$ and the energy of the final state with the full inclusion of the core hole $(\mathrm{FCH})$. Since the binding energy is defined as difference between the finel state ionized (with a core hole) energy of the system and energy of the ground state, we define the chemical shift between $\mathrm{MgO}$ and the other oxides $\mathrm{MO}(\mathrm{M}=\mathrm{Ca}, \mathrm{Sr}$ and $\mathrm{Ba})$ as [378]:

$$
\Delta \mathrm{E}_{\mathrm{MgO}}=\mathrm{E}_{\mathrm{FCH}, \mathrm{MO}}-\mathrm{E}_{\mathrm{NCH}, \mathrm{MO}}-\left(\mathrm{E}_{\mathrm{FCH}, \mathrm{MgO}}-\mathrm{E}_{\mathrm{NCH}, \mathrm{MgO}}\right)
$$

Respect to $\mathrm{MgO}$ (taken as o) we obtain $2.423 \mathrm{eV}$ for $\mathrm{CaO}, 2.958 \mathrm{eV}$ for $\mathrm{SrO}$ and $3.08 \mathrm{eV}$ for $\mathrm{BaO}$. The shift trend suggests that it is easier to remove a oxygen is electron from $\mathrm{MgO}$ respect to the heavier alkaline earth metal oxides. Increasing the difference in electronegativity (see Table 20) with the formation of a core hole stabilizes the final state respect to the initial state and the more electropositive is the metal, the lower is the binding energy. The chemical shift trend found reinforces the hypothesis that the trend in ionicity goes up through the series with $\mathrm{MgO}$ as the most ionic.

If we turn our attention the absorption onset we obtain the opposite trend, the $\mathrm{MgO}$ spectra is blue shifted respect to the heavier oxides in the series 48 . We should anyway keep in mind that the main difference between the chemical shifts obtained as ionized and ground state energy differences between two systems and the absorption onset is that in the XAS framework the final state is in principle charge screened. So the difference in the two trends depends mainly on the different character of the full core hole final state and the XAS excited core hole final state. 


\subsection{CONCLUSIONS}

DFT $\triangle \mathrm{SCF}$ calculations of oxygen $\mathrm{K}$ edge spectra for the system $\mathrm{MgO}, \mathrm{CaO}$, $\mathrm{SrO}$ and $\mathrm{BaO}$ have been performed.

The comparison with experimental data shows a good match for the oxygen $\mathrm{K}$ edge spectra of the $\mathrm{MgO}$ system, where the experimental features are well reproduced, while for the $\mathrm{CaO}, \mathrm{SrO}$ and $\mathrm{BaO}$ system some discrepancies have been highlighted. We attribute the reason of the inconsistencies to $\mathrm{CO}_{2}$ and $\mathrm{H}_{2} \mathrm{O}$ contamination and we also show the corresponding oxygen $\mathrm{K}$ edge spectra for the carbonates and the hydroxides.

In order to analyze the core hole effects we compare the core excited spectra with the spectra obtained with the omission of the core hole treatment. The inclusion of the core hole treatment is necessary for the lighter oxides of the series as $\mathrm{MgO}$ and $\mathrm{CaO}$ while for $\mathrm{SrO}$ and $\mathrm{BaO}$ the no-core-hole spectra already offers an affordable description of the system. The different extent of the core hole effects is linked to different degree of localization in the electron distributions. The lighter oxides of the series show a higher localization and so a lower degree of covalency in the M-O character.

The analysis of the ground state Wannier functions confirms a more delocalized character of states arising from from metal and oxygen $2 p$ hybridization. The shape of the ground state Wannier functions can has been rationalized in terms of hopping integrals where the weight of the $t_{3}$ terms in the hopping process is dominant for the heavier oxides in the series and explains the long tail of px-like oxygen Wannier functions. In order to further investigate the bonding character in the alkaline earth metal oxide series the Bader analysis has been performed and the result indicates a high degree of ionicity for all the systems with $\mathrm{MgO}$ as the most ionic.

The oxygen is chemical shift computed as difference between the energy of the system with a oxygen is core hole and the energy of the ground state reveals a stronger bound oxygen is electron for the heavier oxides in the series respect to $\mathrm{MgO}$, confirming the stronger ionicity character found for the latter. In summary the results reported in this work not only clarify the discrepancy between the available experimental data but further explore the effect of electron localization in the interpretation of spectroscopical data. We can conclude that it is indeed true that all the alkaline earth metal oxides are ionic materials but the covalent component in the $\mathrm{O}-\mathrm{M}$ bond becomes stronger going down through the series. 


\section{AND F-OXIDES}

Solid oxides from d- and f-elements are different from the s- and p-elements because of the presence of $\mathrm{d}$ - and/or f-electrons that have a stronger localisation. One consequence of this localisation is that normal DFT calculations, either band structure or multiple scattering, tend to lead to incomplete descriptions of the electronic structure.

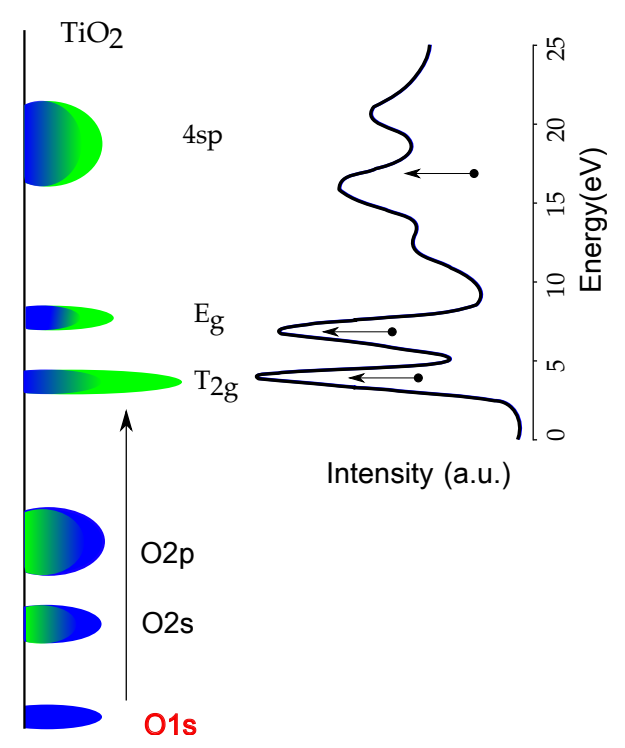

Figure 49: Interpretation of the oxygen K edge XAS spectrum of a $3 \mathrm{~d}$ transition metal oxide. The oxygen 1 core state is given in blue at $530 \mathrm{eV}$ binding energy. The occupied oxygen $2 \mathrm{~s}$ and $2 \mathrm{p}$ bands are given as a combination of oxygen (blue) and metal (green). The empty states are given above the oxygen states: The ratio of $t_{2 g}$ and $e_{g}$ states is 6:4, but the oxygen contribution (in blue) is equivalent. At higher energy the metal $4 \mathrm{sp}$ band is given. The experimental spectrum of $\mathrm{TiO}_{2}$ is given as example.

In the discussion of the $\mathrm{s}$ and $\mathrm{p}$ oxides we have used the identity of the DFT calculated empty states with the XAS spectral shape.

Band theory [381] fails to describe the detailed electronic and magnetic order of $3 \mathrm{~d}$ transition metal oxides, because it neglects the strong local interactions between the $3 \mathrm{~d}$ electrons. The conduction electrons are in bands with mainly $3 \mathrm{~d}$ character from the transition metal ion and the relatively small overlap between the $3 \mathrm{~d}$ orbitals of the metal and the $\mathrm{p}$ orbitals of the oxy- 
gen makes the energy bands narrow, with bandwidths of 1 to $2 \mathrm{eV} \mathrm{[382].}$ The repulsive interaction between $3 \mathrm{~d}$ electrons leads to localization and the formation of non conducting states.

The strongly correlated nature of the 3 d electrons causes the $2 p$ XAS spectra of the $3 \mathrm{~d}$ metals to be dominated by local interactions, implying that the $2 p$ XAS spectral shape has no direct correspondence to the empty states. The main reasons are the strong 2p3d electron-electron interactions (multiplet effects) and the $2 p$ spin-orbit coupling. As discussed in Chapter 1 , for the oxygen $\mathrm{K}$ edge spectra these correlation effects are less important.

There are no multiplet effects, no core hole spin-orbit coupling and the core hole sits on oxygen while the strongly correlated $3 \mathrm{~d}$ states are localised on the metal sites. It turns out to be the case that (within the $0.3 \mathrm{eV}$ resolution as given by the lifetime broadening) the empty states as calculated by LDA+U combined with core-hole methods are still relatively close to the experimental results. The interpretation is schematically indicated in figure 49 .

\subsection{GENERAL CONSIDERATIONS OF 3D-ELEMENT OXIDES}

\subsubsection{The effects of crystal field, exchange and covalency}

We first discuss the general analysis of the oxygen $\mathrm{K}$ edges of $3 \mathrm{~d}$ transition metal oxides.

The crystal field splitting and the exchange splitting are analysed and we look at the influence of the covalency on the trend of the $3 \mathrm{~d}$ series and also on the effect of the covalcy on the spectral shape. Next we discuss binary oxides and their trends in the spectra as a function of oxidation state.

Crystal field splitting: There is a close relation between the energy difference separating the two peaks of the $3 \mathrm{~d}$ band and the ligand field splitting. In case of $3 \mathrm{~d}^{0}$ systems and high-spin $3 \mathrm{~d}^{5}$ systems there is no additional effect due to exchange and the relation between the peak distance and 1oDq should hold exactly. In binary oxides, the crystal field splitting is approximately $0.6 \mathrm{eV}$ times the formal valence, which would imply a crystal field of $1.2 \mathrm{eV}$ in $\mathrm{MnO}$ and $2.4 \mathrm{eV}$ in $\mathrm{TiO}_{2}$, where the actual values can be different by $\tilde{0} .2 \mathrm{eV}$. Comparing the peak distance to the $10 \mathrm{Dq}$ value as determined from optical dd-excitations, one finds that the oxygen $\mathrm{K}$ edge spectra yield a $10 \%$ shorter energy distance [383]. The final state core hole localizes the oxygen $2 p$ states, thereby decreasing the overlap with the metal $3 \mathrm{~d}$ states. We note that this reduction is less than for the metal $2 p$ XAS spectra that 
have a final state $10 D q$ value that is reduced by $\tilde{2} 0 \%$ via a similar core hole localisation effect [384].

Exchange splitting: In systems with a finite spin there is a splitting between the spin-up and spin-down sub-bands.

For example high-spin octahedral $3 \mathrm{~d}^{3}$ systems have three spin-up $t_{2 g}$ electrons in their ground state. This creates an exchange splitting between the empty high-spin and low-spin states. The exchange splitting can be approximated as the number of unpaired spins times $0.6 \mathrm{eV}$. This implies that in case of $\mathrm{Cr}_{2} \mathrm{O}_{3}$ the exchange splitting is $\tilde{\mathrm{I}} .8 \mathrm{eV}$ and also the crystal field is $\tilde{\mathrm{i}} .8$ $\mathrm{eV}$.

Covalency and the trend of the $3 \mathrm{~d}$ series: Looking at the oxygen $\mathrm{K}$ edge of binary $3 \mathrm{~d}$ oxides where the metal ions have an octahedral surroundings, one finds a similar shape of the empty states due to interaction of the metal $4 \mathrm{~s}$ and $4 \mathrm{p}$ and oxygen $2 \mathrm{p}$ states. The lowest energy states are the oxygen $2 \mathrm{p}$ states that are hybridised with the empty 3 d states.

Assuming an equal hybridization for all systems, the intensity of the $3 \mathrm{~d}$ band yields the number of empty 3 d states. Analysis shows that one finds approximately a linear relation between the intensity of the $3 \mathrm{~d}$ band and the number of empty $3 \mathrm{~d}$ states [337], where the $3 \mathrm{~d}$ band intensity is normalised to the intensity of the $4 \mathrm{sp}$ band. Suntivich et al. found for a series of perovskites that the covalency is not constant but becomes larger through the $3 \mathrm{~d}$-series [385]. While filling the $3 \mathrm{~d}$-band the energy-position of the $3 \mathrm{~d}$-states with respect to the oxygen $2 p$ states decreases, thereby increasing their overlap. In addition, the average metal-oxygen distance gets shorter implying

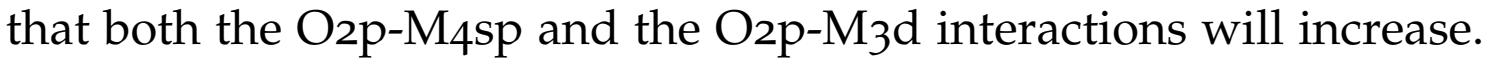

The average metal-oxygen distance varies between different oxide systems, for example $\mathrm{M}_{2} \mathrm{O}_{3}$ corundum has larger metal-oxygen distances than $\mathrm{LaMO}_{3}$ perovskites. This variation can cause differences in the intensity of the $3 \mathrm{~d}-$ part of the spectrum even for the same element, valence and spin-state.

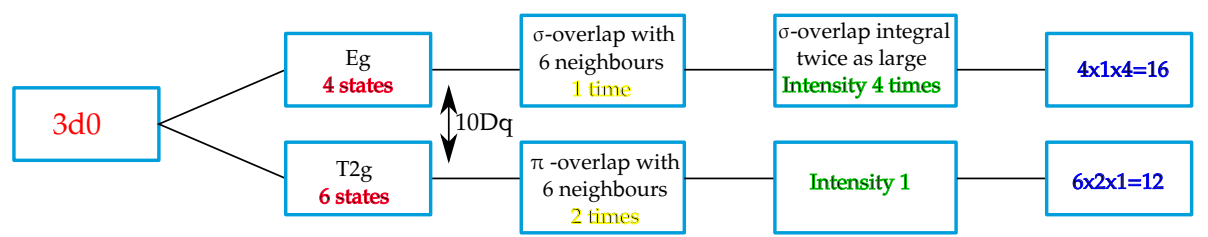

Figure 5o: Intensity of the $t_{2 g}$ and $e_{g}$ peaks of a $3 d^{0}$-system due to degeneracy, number of overlaps and overlap strengths. 
Covalency and the spectral shape: The covalency can be further analysed by including the difference between the hybridization of the $t_{2 g}$ and the $e_{g}$ states in octahedral symmetry.

The $e_{g}$ states point directly to the oxygen atoms and have $\sigma$ overlap. The $t_{2 g}$ states point in between the oxygen atoms and have $\pi$ overlap with the oxygen $2 p$ states. Effectively this yields a difference in the hopping terms of 2:1, which implies a difference in hybridization of 4:1 for each $\mathrm{O}_{2} \mathrm{p}-\mathrm{M}_{3} \mathrm{~d}$ overlap.

Additional factors for the intensity ratio are the fact that the $\pi$ bonding is possible with two $\mathrm{O}_{2} \mathrm{p}$ orbitals and $\sigma$ overlap with one oxygen $2 \mathrm{p}$ orbital. In case of $3 \mathrm{~d}^{0}$ systems $\left(\mathrm{TiO}_{2}\right)$ there are six $\mathrm{t}_{2 g}$ and four $\mathrm{e}_{\mathrm{g}}$ states. The total (relative) intensity ratio for the $t_{2 g} / e_{g}$ band is $1 / 4 *^{*} / 1 * 6 / 4=12 / 16$. This implies that one expects a 3:4 integrated intensity ratio for the $t_{2 g}$ to $e_{g}$ band, which is close to the observed ratio for $\mathrm{TiO}_{2}$ and $\mathrm{MnO}$.

Figure 50 shows the effects of degeneracy and overlap on the $t_{2 g}$ and $e_{g}$ peaks of a $3 \mathrm{~d}^{0}$-system. In case of $3 \mathrm{~d}^{1}$ systems one can assume that the exchange splitting is smaller than the crystal field splitting, and with this assumption the $t_{2 g}: e_{g}$ band ratio is 10 to 16 .

The $3 \mathrm{~d}^{3}$ system has an exchange splitting that is 1.5 to $2.0 \mathrm{eV}$, which implies

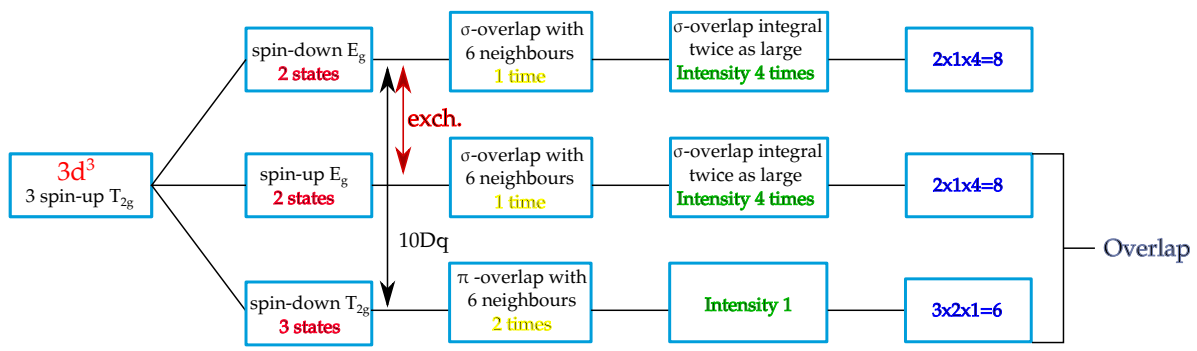

Figure 51: Intensity of the $t_{2 g}$ and $e_{g}$ peaks of a $3 d^{3}$-system due to the exchange splitting, degeneracy, the number of overlaps and the overlap strengths.

that the spin-down $t_{2 g}$ band overlaps with the spin-up $e_{g}$ band, which complicates the two-band $t_{2 g}: e_{g}$ analysis. Figure 51 shows the effects of exchange splitting, degeneracy and overlap on the $t_{2 g}$ and $e_{g}$ peaks of a $3 \mathrm{~d}^{3}$-system.

\subsubsection{Binary oxides and oxidation state trends}

We first discuss the trends of the oxygen $\mathrm{K}$ edge through the $3 \mathrm{~d}$ series will be discussed on three series of binary oxides, respectively the rocksalt $\mathrm{MO}$ oxides, the corundum $\mathrm{M}_{2} \mathrm{O}_{3}$ oxides and the rutile $\mathrm{MO}_{2}$ oxides. 
Figure 52 shows the divalent $3 \mathrm{~d}$ transition metal oxides with a rocksalt structure. respectively given are $3 \mathrm{~d}^{3} \mathrm{VO}, 3 \mathrm{~d}^{5} \mathrm{MnO}, 3 \mathrm{~d}^{6} \mathrm{FeO}, 3 \mathrm{~d}^{7} \mathrm{CoO}$ and $3 \mathrm{~d}^{8} \mathrm{NiO}$, where these divalent $3 \mathrm{~d}$ transition metal oxides are always highspin. They all have a crystal field splitting of approximately $1.2 \mathrm{eV}$. The lowest energy peaks relates to the empty $3 \mathrm{~d}$ states and the peaks around $540 \mathrm{eV}$ relate the the metal $4 \mathrm{~s}$ and $4 \mathrm{p}$ band.

Rocksalt has a typical sharp band structure peak around $545 \mathrm{eV}$. In case of $\mathrm{MnO}$ the empty $3 \mathrm{~d}$ states are at relatively high-energy at $534 \mathrm{eV}$ and they overlap with the $4 \mathrm{~s}$ and $4 \mathrm{p}$ structures. $\mathrm{MnO}$ has a $3 \mathrm{~d}^{5}$ configuration and in principle one would expect two $3 \mathrm{~d}$ peaks split by the crystal field. Because of the small crystal field splitting of $1.2 \mathrm{eV}$, compared with the band widths, the splitting is not visible in the spectral shape.

$\mathrm{FeO}$ has a $3 \mathrm{~d}^{6}$ configuration and similar to $\mathrm{MnO}$ the crystal field splitting is too small to be visible.

In case of $\mathrm{CoO}$ one could see fine structure in the $3 \mathrm{~d}$ peak and the first small feature could be assigned to the empty $t_{2 g}$ state. NiO has a $3 d^{8}$ configuration spin-down $e_{g}$ band is empty, which yields a sharp $3 \mathrm{~d}$ peak.

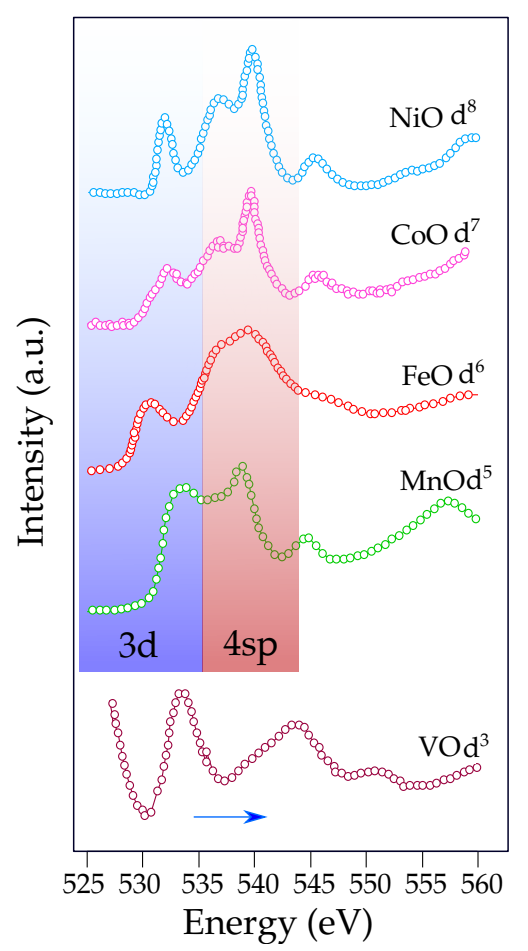

Figure 52: Oxygen $\mathrm{K}$ edge of the $3 \mathrm{~d}$ monoxides $\mathrm{VO}$ [386], $\mathrm{MnO}$ [387], $\mathrm{FeO}$ [388], $\mathrm{CoO}[389]$ and $\mathrm{NiO}$ [389]. Below 535 the $3 \mathrm{~d}$-band related peaks and from 536 to $543 \mathrm{eV}$ the metal $4 \mathrm{sp}$ band. In case of $\mathrm{VO}$, the peaks appear at higher energy. 
Figure 53 shows the trivalent $3 \mathrm{~d}$ transition metal oxides with a corundum structure. Respectively given are $3 \mathrm{~d}^{1} \mathrm{Ti}_{2} \mathrm{O}_{3}, 3 \mathrm{~d}^{2} \mathrm{~V}_{2} \mathrm{O}_{3}, 3 \mathrm{~d}^{3} \mathrm{Cr}_{2} \mathrm{O}_{3}, 3 \mathrm{~d}^{4}$ $\mathrm{Mn}_{2} \mathrm{O}_{3}, 3 \mathrm{~d}^{5} \mathrm{Fe}_{2} \mathrm{O}_{3}$ and $3 \mathrm{~d}^{6} \mathrm{Co}_{2} \mathrm{O}_{3}$. All trivalent $3 \mathrm{~d}$ transition metal oxides have a crystal field splitting of $1.8 \mathrm{eV}$ and are high-spin, except $\mathrm{Co}_{2} \mathrm{O}_{3}$ that is low-spin.

The $3 \mathrm{~d}^{1}$ system $\mathrm{Ti}_{2} \mathrm{O}_{3}$ has two peaks in the $3 \mathrm{~d}$ part split by the crystal field. Trivalent oxides have a crystal field splitting of 1.8 to $2.0 \mathrm{eV}$, clearly visible in the oxygen $\mathrm{K}$ edges.At $540-545 \mathrm{eV}$ the structure related to the metal $4 \mathrm{~S}$ and $4 \mathrm{p}$ bands is visible. The $3 \mathrm{~d}^{2}$ system $\mathrm{V}_{2} \mathrm{O}_{3}$ has a similar spectral shape. In case of the $3 \mathrm{~d}^{3}$ system $\mathrm{Cr}_{2} \mathrm{O}_{3}$ the exchange splitting between spin-up and spin-down states is similar in size to the crystal yielding a first intense peak related to a combination of the spin-down $t_{2 g}$ and spin-up $e_{g}$ band.

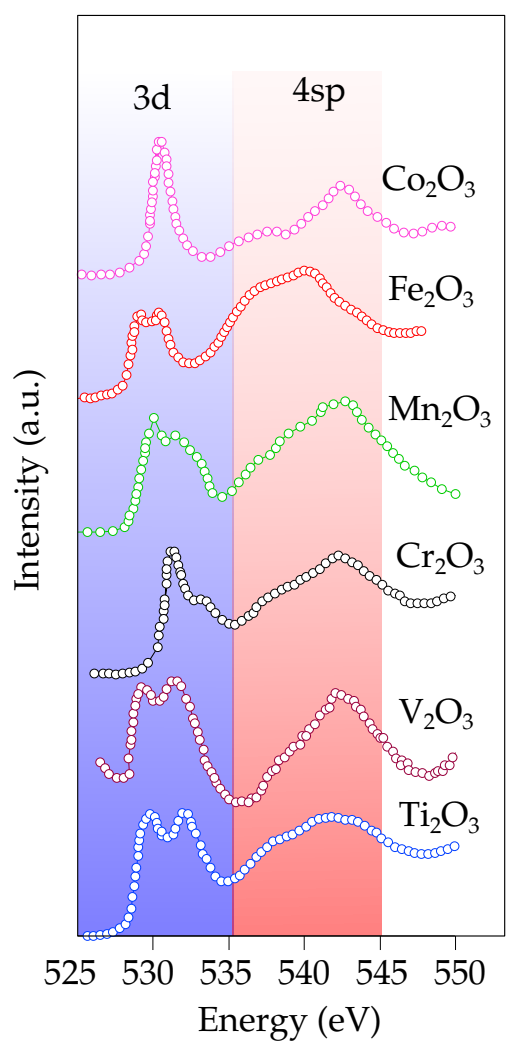

Figure 53: Oxygen $\mathrm{K}$ edge of the corundum oxides $\mathrm{Ti}_{2} \mathrm{O}_{3}$ [337], $\mathrm{V}_{2} \mathrm{O}_{3}$ [337], $\mathrm{Cr}_{2} \mathrm{O}_{3}$ [337], $\mathrm{Mn}_{2} \mathrm{O}_{3}$ [390], $\mathrm{Fe}_{2} \mathrm{O}_{3}$ [337] and $\mathrm{Co}_{2} \mathrm{O}_{3}$ [391]. Below 535 the $3 \mathrm{~d}$-band related peaks and from 536 to $545 \mathrm{eV}$ the metal $4 \mathrm{sp}$ band

The $3 \mathrm{~d}^{4}$ system $\mathrm{Mn}_{2} \mathrm{O}_{3}$ is a Jahn-Teller system due to its half-filled spin=up $\mathrm{e}_{\mathrm{g}}$ state. The combination of (i) the octahedral crystal field, (ii) the exchange splitting and (iii) the Jahn-Teller distortion yields a broad 3 d-related feature. The $3 \mathrm{~d}^{5}$ system $\mathrm{Fe}_{2} \mathrm{O}_{3}$ has a half-filled $3 \mathrm{~d}$-band and shows two peaks split 
by the crystal field splitting.

The $3 \mathrm{~d}^{6}$ system $\mathrm{Co}_{2} \mathrm{O}_{3}$ is low-spin and has a filled $\mathrm{t}_{2 \mathrm{~g}}$ band, hence a single sharp peak related to th empty $\mathrm{e}_{\mathrm{g}}$ band.

Figure 54 shows the tetravalent $3 \mathrm{~d}$ transition metal oxides with a rutile structure. Respectively are given $3 \mathrm{~d}^{0} \mathrm{TiO}_{2}, 3 \mathrm{~d}^{1} \mathrm{VO}_{2}, 3 \mathrm{~d}^{2} \mathrm{CrO}_{2}$ and $3 \mathrm{~d}^{3} \mathrm{MnO}_{2}$, where these tetravalent $3 \mathrm{~d}$ transition metal oxides have a crystal field splitting of $2.4 \mathrm{eV}$.

$\mathrm{TiO}_{2}$ shows two peaks split by the crystal field splitting and the $\mathrm{VO}_{2}$ spectrum is similar, but due to the presence of the vanadium $\mathrm{L}_{2,3}$ edge at 720 $\mathrm{eV}$, the oxygen $\mathrm{K}$ edge of $\mathrm{VO}_{2}$ sits on a background of the tail of this spectrum. The $3 \mathrm{~d}^{2}$ system $\mathrm{CrO}_{2}$ is a well-known half-metal. The relatively large exchange splitting yields a first spin-up $t_{2 g}$ band, while the other sub-bands are all part of the second structure. The $3 \mathrm{~d}^{3}$ system $\mathrm{MnO}_{2}$ has a similar spectrum as $\mathrm{Cr}_{2} \mathrm{O}_{3}$, with the first peak a mixture of the spin-down $\mathrm{t}_{2 \mathrm{~g}}$ and spin-up $e_{g}$ band.

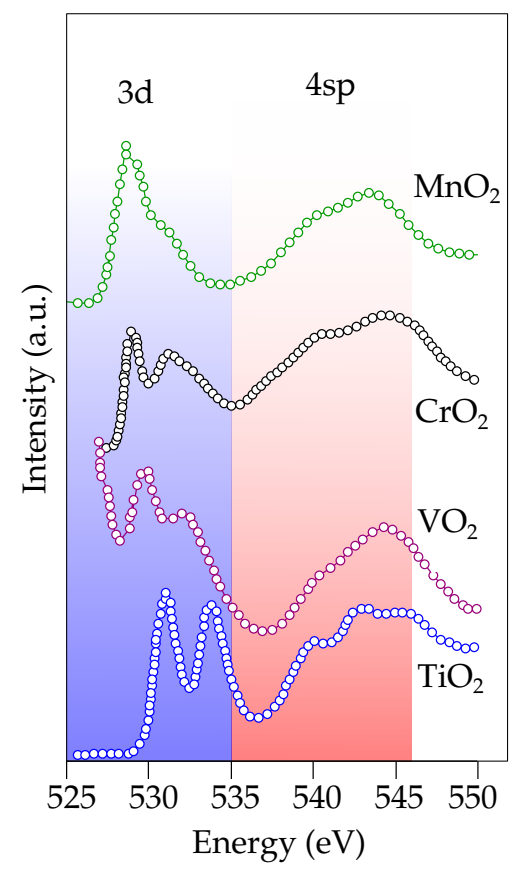

Figure 54: Oxygen $\mathrm{K}$ edge of the rutile oxides $\mathrm{TiO}_{2}$ [389], $\mathrm{VO}_{2}$ [386], $\mathrm{CrO}_{2}$ [392] and $\mathrm{MnO}_{2}$ [387]. Below 535 the $3 \mathrm{~d}$-band related peaks and from 536 to 547 $\mathrm{eV}$ the metal $4 \mathrm{sp}$ band.

The comparison of the three common valences $2+, 3+$ and $4+$ gives rise to the following observations:

Intensity of the $3 \mathrm{~d}$-part: The $3 \mathrm{~d}$-part of the spectrum has more intensity for high-valence oxides. The reason is that the covalency increases when the 
valence is increased from $2+$ to $3^{+}$and $4^{+}$.

Increased covalence implies more oxygen character in the metal $3 \mathrm{~d}$-band and as such more intensity in the $3 \mathrm{~d}$-part of the oxygen $\mathrm{K}$ edge. Due to the increased covalency also the 4sp-part of the spectrum will gain intensity but this effect is smaller than for the $3 \mathrm{~d}$-part because the $4 \mathrm{~s}$ and $4 \mathrm{p}$ orbitals are already delocalized and gain less overlap than the $3 \mathrm{~d}$ orbitals.

Excitation energy: The excitation energy of the metal $\mathrm{L}_{2,3}$ edges shifts to higher energy with increased valence. The simplest approximation is that is due to the increased (effective) charge on the metal site, implying a larger energy needed to excite a core electron. In contrast, the energy position of the oxygen $\mathrm{K}$ edge does not always shift in the same direction by changing the formal charge of the metal ion. Depending on the specific metal ion the oxygen $\mathrm{K}$ edge shifts to lower energy, to higher energy of remains at the same energy.

Looking at the experimental data there is no shift observed for the valence changes $\mathrm{Ti}^{3+} / \mathrm{Ti}^{4+}, \mathrm{V}^{3+} / \mathrm{V}^{4+} / \mathrm{V}^{5+}$ and $\mathrm{Mn}^{3+} / \mathrm{Mn}^{4+}$. In some cases the increase in valence opens a sub-band that was occupied with the lower valence, with the result that the oxygen $\mathrm{K}$ edge shifts to lower excitation energy. This happens for the octahedral $3 \mathrm{~d}^{3}, 3 \mathrm{~d}^{5}$ and $3 \mathrm{~d}^{8}$ systems, including $\mathrm{Ni}^{2+} / \mathrm{Ni}^{3+}$ and $\mathrm{Fe}^{3+} / \mathrm{Fe}^{4+}$. These two examples also contain a negative $\Delta$ ion (i.e. a system where the charge transfer energy $(\Delta)$ is smaller than zero, implying a significant amount of holes on the oxygen ions). A negative $\Delta$ is a second reason for a shift to lower energy and the couple $\mathrm{Cu}^{2+} / \mathrm{Cu}^{3+}$ shows a shift to lower energy although no new sub-band becomes available in the low-spin negative $\Delta \mathrm{Cu}^{3+}$ oxides.

Finally a change of spin-state from high-spin to low-spin can cause a positive shift as visible for $\mathrm{Co}^{2+} / \mathrm{Co}^{3+}$ (in cases where the $\mathrm{Co}^{3+}$ system is lowspin). In conclusion we state that there is no uniform rule regarding the shift of the excitation energy with valence.

A last point of warning is that the energy calibration of many oxygen $\mathrm{K}$ edges is not uniform throughout the literature.

Crystal field: As mentioned above, the crystal field in octahedral highspin oxides follows the approximate rule that it is equal to $0.6 \mathrm{eV}$ times the formal valence, implying a crystal field of $1.2 \mathrm{eV}$ for $2+$ oxides, $1.8 \mathrm{eV}$ for $3^{+}$ oxides and $2.4 \mathrm{eV}$ for $4+$ oxides.

The exchange splitting for $3 \mathrm{~d}$-oxides is approximately $0.6 \mathrm{eV}$ per paired spin- 
couple. Taken together these numbers imply that divalent oxides are always high-spin, tetravalent oxides are always low-spin and trivalent oxides have both options, where $\mathrm{Mn}^{3+}, \mathrm{Co}^{3+}$ and $\mathrm{Ni}^{3+}$ are in many oxides close to a high-spin low-spin transition point.

\subsubsection{Perouskites}

Perovskites have a general formula $\mathrm{ABO}_{3}$ where $\mathrm{A}$ is a larger cation than $\mathrm{B}$ and arrange in the ideally cubic structure illustrated in Figure 55a.

Almost all metallic elements from the periodic table are stable in this structure. Depending on the chemical formulation, distortions from the ideal cubic structure might happen.

Another related family of materials are the layered perovskites, which contain layers of perovskite-like arrangement of $\mathrm{MO}_{6}$ metal cation units. One simple example is $\mathrm{A}_{2} \mathrm{BO}_{4}$, which structure is illustrated in Figure 55b. Depending on the chemical composition, various element substitution allow tuning the electron or hole doping in the structure and the resulting properties.

Perovskite and layered perovskite materials are intensively studied from their chemical [393] (heterogeneous catalysis, surface chemistry) and physical properties (electric, magnetic, and optical properties). The layered structure results in enhanced electron mobility and can give rise to specific properties such as superconductivity.

Oxygen K edge spectra have been largely used to probe the M-O chemical
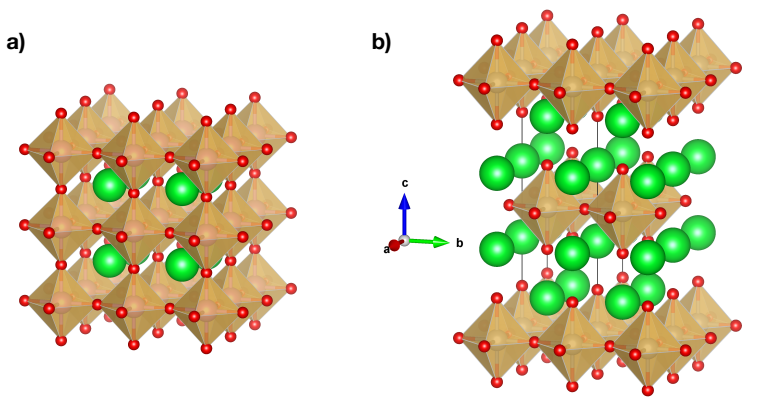

Figure 55: a) Crystal structure of cubic perovskite with the $\mathrm{ABO}_{3}$ formula; b) Crystal structure of layered perovskite with the $\mathrm{A}_{2} \mathrm{BO}_{4}$ formula. A: green ; $\mathrm{B}$ : orange, O: red.

bonding, or the metal valence and spin state although it often requires complementary measurements such as the metal $\mathrm{L}_{2,3}$ edges.

Suntivich et al. performed a systematic analysis of the chemical bonding of 
$\mathrm{LaTMO}_{3}$ systems based on the oxygen $\mathrm{K}$ edge [385], for the transition metals $\mathrm{Cr}, \mathrm{Mn}, \mathrm{Fe}, \mathrm{Co}$ and $\mathrm{Ni}$. The oxygen $\mathrm{K}$ edge spectra of the series extended with the other $3 \mathrm{~d}$ metals from complementary references is shown in Figure 56 .

The perovskite spectra show a similar general shape with a first single or double peak corresponding to the oxygen $2 p$ states hybridized with the metal $3 \mathrm{~d}$ states. The shape of this features is correlated to the valency and spin state of the transition metal ion. At $535 \mathrm{eV}$ one finds the oxygen $2 \mathrm{p}$ states hybridized with the lanthanide $5 \mathrm{~d}$ states followed by the oxygen $2 p$ states hybridized with the metal 4 sp states.

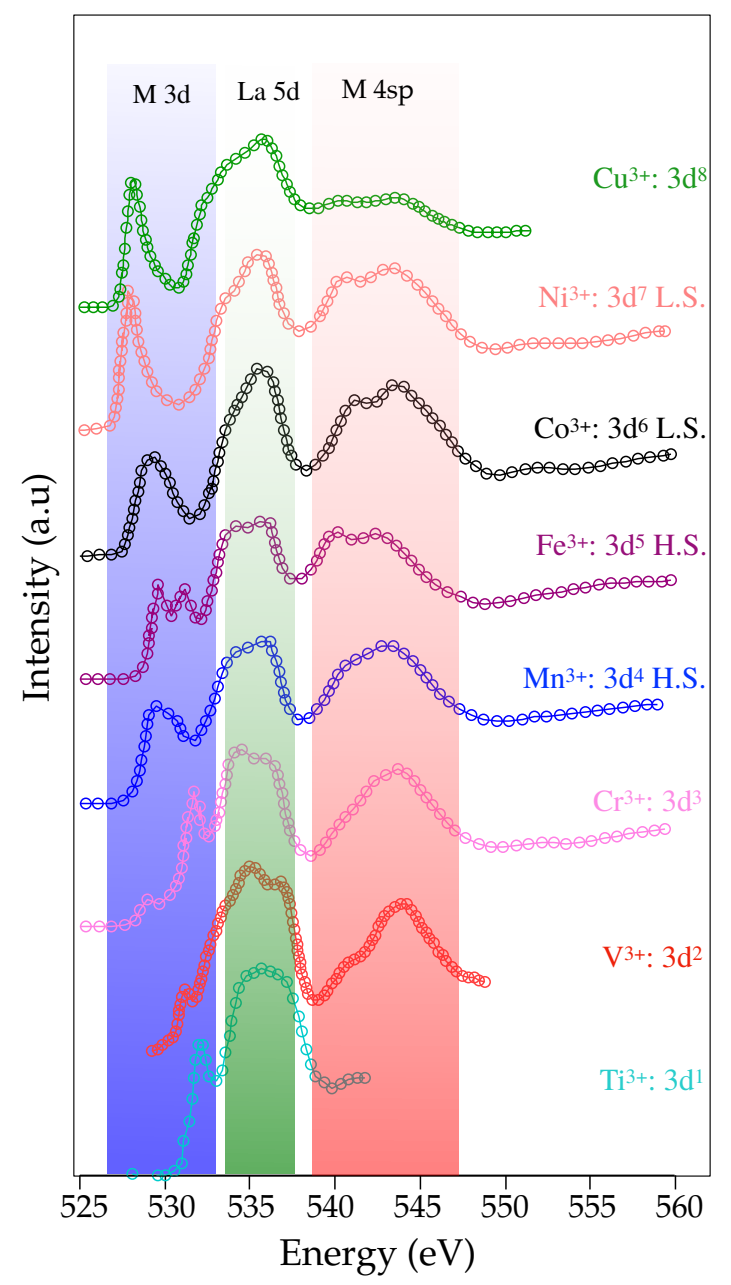

Figure 56: Oxygen $\mathrm{K}$ edge spectra of $\mathrm{LaMO}_{3}$ perovskite systems for the transition metals Ti [394], V [395], Cr [385], Mn [385], Fe [385], Co [385], Ni [385] and $\mathrm{Cu}$ [396]. (H.S.: high spin; L.S.: low spin) 
Covalency in $\mathrm{d}^{0}$ tetrahedral oxyanions The isoelectronic $\mathrm{d}^{0}$ metal cations from Group 6 and 7 (Cr, Mo, W, Mn, Tc, Re) form tetrahedral oxyanions $\mathrm{MO}_{4}^{\text {n- }}$. The investigation of this series allows probing the metal-oxygen bond covalency trends. A first comparative study of the oxyanions $\mathrm{MO}_{4}(\mathrm{M}=\mathrm{Ti}, \mathrm{V}, \mathrm{Cr}, \mathrm{Mn})$ was done by Brydson et al. [397] who proposed a rationalization of the observed trends in both metal $\mathrm{L}_{2,3}$ edge and oxygen $\mathrm{K}$ edge. Minasian et al. performed this comparative study, including the radioactive Tc compound, using oxygen $\mathrm{K}$ edges with three different detection methods, respectively transmission XAS in a STXM, TFY XAS and IXS and compared the experiments with TD-DFT calculations [398]. In an earlier study Bradley et al. had also compared the oxygen K edge spectra obtained from different methods[399].

Figure 57 shows the oxygen K edge spectra of the series. The first two peaks in the spectra are respectively the transition to the empty $e$ and $t_{2}$ states, split by approximately $2.0 \mathrm{eV}$ in all six systems. The excitation energy of the first peak is lowest in the $3 \mathrm{~d}$ systems, followed by $4 \mathrm{~d}$ and $5 \mathrm{~d}$. In addition the $\mathrm{Mn}$, Tc and Re oxyanions have lower excitation energies than their $\mathrm{Cr}$, Mo and $\mathrm{W}$ analogs. There is a linear relation between the first peak in the oxygen $\mathrm{K}$ edge and the first optical excitation energy, i.e. from the highest occupied state to the empty $e$ states. Minasian et al. demonstrated that the $e$ ( $\pi$ overlap) mixing increases from group 6 to 7 and down the periods while the $t_{2}$ symmetry ( $\pi+\sigma$ overlap) mixing is rather constant. TD-DFT simulations of spectra are in good agreement with the experimental spectra.

\subsection{OVERVIEW OF THE 3D OXIDES}

In the following sections the $3 \mathrm{~d}$ transition metal oxides are discussed by element.

\subsubsection{Titanium oxides}

Most titanium oxides have a valency of $4^{+}$, implying an empty $3 \mathrm{~d}$ band. They are insulators with a band gap between the oxygen $2 p$ band and the titanium $3 \mathrm{~d}$ band. The binary oxide $\mathrm{TiO}_{2}$ occurs in three different crystal structures, respectively rutile, anatase and brookite.

Its oxygen $\mathrm{K}$ edge has been measured many times [337, 363, 383, 401-406]. $\mathrm{Ti}^{4+}$ forms perovskite structures with $\mathrm{Ba}^{2+}, \mathrm{Ca}^{2+}$ and $\mathrm{Sr}^{2+} . \mathrm{SrTiO}_{3}$ is commonly used support material for thin film oxides. Figure 58 shows the oxygen $\mathrm{K}$ edge of the perovskites $\mathrm{SrTiO}_{3}$ and $\mathrm{LaTiO}_{3}$. The 3d peaks in the oxygen $\mathrm{K}$ edge are essentially given by the valency of titanium, $4+$ for $\mathrm{SrTiO}_{3}$ and $3+$ for $\mathrm{LaTiO}_{3}$. Because the coordination in the perovskites is close to 

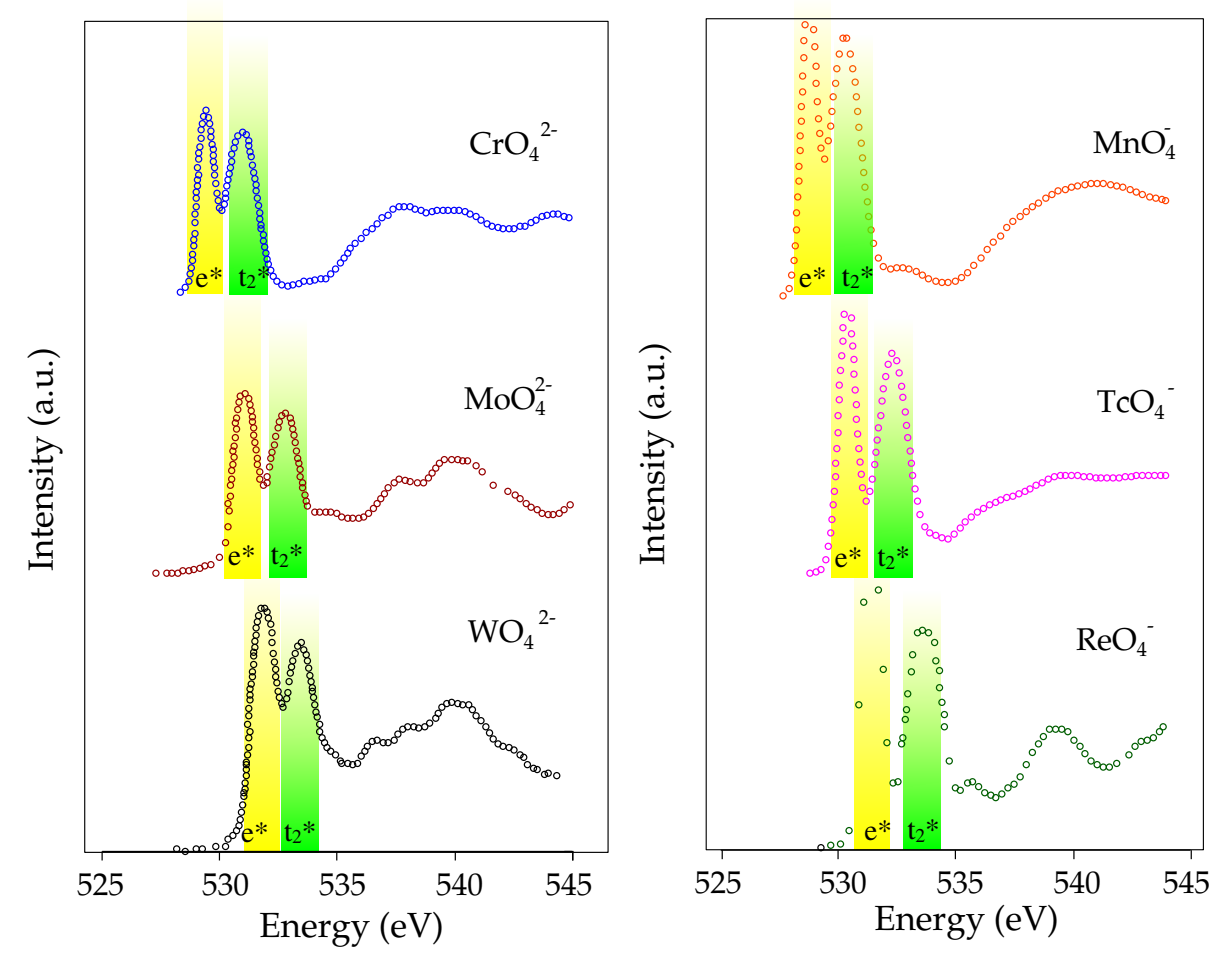

Figure 57: The oxygen $\mathrm{K}$ edge spectra of the oxyanions $\mathrm{MO}_{4}^{\mathrm{n}-}$ with $\mathrm{M}=\mathrm{Cr}, \mathrm{Mo}, \mathrm{W}$, $\mathrm{Mn}, \mathrm{Tc}, \operatorname{Re}[398]$.

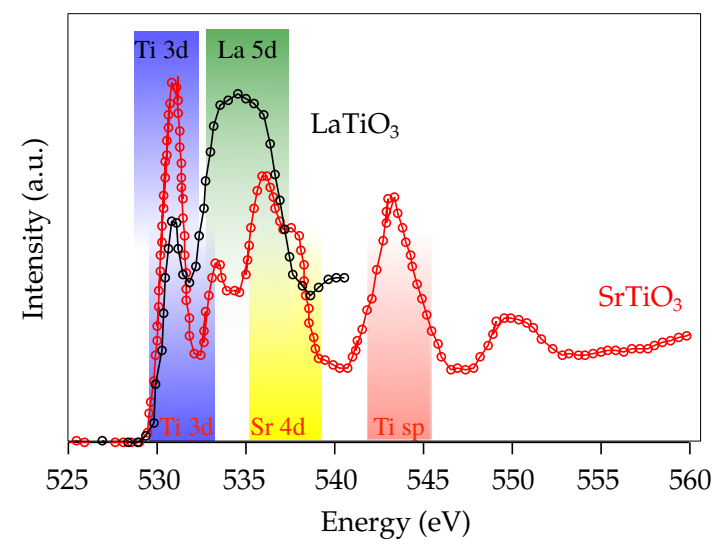

Figure 58: Oxygen K edge of $\mathrm{LaTiO}_{2}$ [40o] and $\mathrm{SrTiO}_{3}$ [394] perovskites. The titanium $3 \mathrm{~d}$ lanthanum $5 \mathrm{~d} /$ strontium $4 \mathrm{~d}$ and titanium 4 sp related peaks are indicated. 
octahedral the $\mathrm{e}_{\mathrm{g}}$-band is relatively wide.

The published oxygen $\mathrm{K}$ edges of $\mathrm{SrTiO}_{3}$ shows some variation in the intensity of the $\mathrm{e}_{\mathrm{g}}$-peak $[383,405,407,408]$. The oxygen $\mathrm{K}$ edge of $\mathrm{CaTiO}_{3}[404]$ and $\mathrm{BaTiO}_{3}$ [406] are equivalent to that of $\mathrm{SrTiO}_{3}$. The best resolution oxygen $\mathrm{K}$ edge of $\mathrm{SrTiO}_{3}$ is given by Palina et al. [394]. Palina et al. compare pure $\mathrm{SrTiO}_{3}$ with $\mathrm{TiO}_{2}$-terminated $\mathrm{SrTiO}_{3}$ and thin films/interfaces with $\mathrm{LaAlO}_{3}$. They claim that for the conducting samples, a new peak is visible at $540.5 \mathrm{eV}$ due to "molecular-like oxygen", related to the concentration of electronic defects.

The trivalent titanium oxides $\mathrm{Ti}_{2} \mathrm{O}_{3}[337,404]$ and $\mathrm{LaTiO}_{3}[407]$ have a $3 \mathrm{~d}^{1}$ ground state. Haverkort et al. performed a detailed analysis of the ground state [409] based on the titanium L edge. They found that the ground state is affected by the crystal field distortion and the $3 \mathrm{~d}$ spin-orbit coupling. Such details cannot be found from the oxygen $\mathrm{K}$ edge due to the lack of final state interactions. Fujimori et al. studied the effects of doping from the oxygen $\mathrm{K}$ edge spectra of a series of $(\mathrm{La}, \mathrm{Sr}) \mathrm{TiO}_{3}$ systems [400]. While electron doping creates a pre-peak for the late transition metal ions such as in the cuprates, this is not the case for the $\mathrm{Ti}^{+3} / \mathrm{Ti}^{4+}$ system. The first $\mathrm{t}_{2 \mathrm{~g}}$-peak in the oxygen $\mathrm{K}$ edge has approximately the same energy for $\mathrm{LaTiO}_{3}$ and $\mathrm{SrTiO}_{3}$, which is related to the large covalency of the titanium systems and the fact that the Hubbard $U$ is smaller than the charge transfer energy.

\subsubsection{Vanadium oxides}

The vanadium $2 \mathrm{p}$ XAS edge sits at $515 \mathrm{eV}$ which implies that the oxygen $\mathrm{K}$ edge of vanadium oxides is found in the tail of the vanadium $\mathrm{L}_{2,3}$ edge. This complicates the analysis as the background of the vanadium $L_{2,3}$ edge must be subtracted. In the 1989 paper on transition metal oxides the spectrum of $\mathrm{V}_{2} \mathrm{O}_{3}$ and $\mathrm{VO}_{2}$ has been included [337].

A systematic study of the oxygen $\mathrm{K}$ edges of vanadium oxides has been published by Abbate et al. [410]. They compared the spectra of $\mathrm{V}_{2} \mathrm{O}_{3}, \mathrm{VO}_{2}$ and $\mathrm{V}_{2} \mathrm{O}_{5}$ with the projected DOS calculated with the LSW method.

Goering et al. measured $\mathrm{V}_{2} \mathrm{O}_{3}, \mathrm{VO}_{2}, \mathrm{~V}_{2} \mathrm{O}_{5}$ and also the mixed valent oxide $\mathrm{V}_{6} \mathrm{O}_{13}$. They measured a strong angular dependence in the oxygen $\mathrm{K}$ edge of $\mathrm{V}_{2} \mathrm{O}_{5}$ [411]. Hebert et al. measured the $\mathrm{VO}, \mathrm{V}_{2} \mathrm{O}_{3}, \mathrm{VO}_{2}$ and $\mathrm{V}_{2} \mathrm{O}_{5}$ oxides with EELS and performed Wien97 calculations of the vanadium oxides [386]. In the calculations the core hole was not included.

$\mathrm{V}_{2} \mathrm{O}_{3}$ has a $3 \mathrm{~d}^{2}$ ground state with a partly filled spin-up $\mathrm{t}_{2 \mathrm{~g}}$ shell; it has two phase transitions at approximately $150 \mathrm{~K}$ and $250 \mathrm{~K}$. Below $150 \mathrm{~K} \mathrm{~V}_{2} \mathrm{O}_{3}$ is 
an antiferromagnetic insulator, between $150 \mathrm{~K}$ and $250 \mathrm{~K}$ a (paramagnetic) metal and above $250 \mathrm{~K}$ an insulator again. Park et al. performed detailed vanadium $\mathrm{L}_{2,3}$ XAS and linear dichroism for three different phases of $\mathrm{V}_{2} \mathrm{O}_{3}$ as a function temperature [412]. This revealed the details of the electronic ground states. The oxygen $\mathrm{K}$ edges measured by Park can be found in a paper by Huang et al. [413]. The first peak related to the transition to the $\mathfrak{t}_{2 \mathrm{~g}}$-up state is relatively low in intensity [386, 411, 413], much lower than the $3 \mathrm{~d}^{2}$ system $\mathrm{CrO}_{2}$ (see below). The increased valence of $\mathrm{Cr}^{4+}$ drastically increases the overlap of the $t_{2 g}$ states with the oxygen states.

$\mathrm{VO}_{2}$ has a $3 \mathrm{~d}^{1}$ ground state with a partly filled spin-up $t_{2 g}$ shell; it has a metal-insulator transition and the differences in the electronic structure below and above the phase transition has been studied with the oxygen $\mathrm{K}$ edge [414]. In the insulating phase the $t_{2 g}$-band is split into two sub-bands. Abe et al. measured the $\mathrm{VO}_{2}$ phase transition with EELS [415]. Room temperature spectra of $\mathrm{VO}_{2}$ have also been published by van Aken et al. [404] and Liang et al performed detailed calculations that are discussed in Chapter 1 [408]. $\mathrm{V}_{2} \mathrm{O}_{5}$ has a $3 \mathrm{~d}^{0}$ ground state and is a semi-conductor with a band gap between the oxygen $2 \mathrm{p}$ band and the vanadium $3 \mathrm{~d}$ band. $\gamma-\mathrm{V}_{2} \mathrm{O}_{5}$ has two different vanadium surroundings and five differently coordinated oxygen atoms. The spectrum is different from $\alpha-\mathrm{V}_{2} \mathrm{O}_{5}$ but well reproduced from DFT calculations [416].

Maganas et al. calculated the oxygen $\mathrm{K}$ edge of $\mathrm{V}_{2} \mathrm{O}_{5}$ using a cluster-based calculation. The important feature in their calculation is that they also calculated the vanadium L edge using a Restricted Open shell Configuration Interaction with Singles (ROCIS) method [417].

Lithium doped vanadium oxides are potential battery materials. The oxygen $\mathrm{K}$ edges of $\mathrm{Li}_{1+x} \mathrm{~V}_{3} \mathrm{O}_{8}$ oxides have been measured by Choi et al. [418]. The spectra revealed that the insertion of lithium affects the $\mathrm{V}-\mathrm{O}$ bonds and induces a change in bonding geometry.

Willinger et al. measured the oxygen $\mathrm{K}$ edge of the vanadium phosphate $\mathrm{VOPO}_{4}$ [419]. The $\mathrm{V}^{4+}$ ions sit in a distorted octahedral site. The 3d-part of the spectrum looks similar to $\mathrm{V}_{2} \mathrm{O}_{5}$ and at higher energy the oxygen $\mathrm{K}$ edge is dominated by phosphor-oxygen bonds. The oxygen $\mathrm{K}$ edge of vanadium bronzes have been measured with XAS and with EELS [420]. The $\mathrm{Na}_{0.33} \mathrm{~V}_{2} \mathrm{O}_{5}$ spectrum is calculated with Wien2K calculations without core hole. The $\mathrm{AlV}_{2} \mathrm{O}_{4}$ spinel system is measured with EELS and compared with Wien $2 \mathrm{~K}$ calculations. In contrast to the binary oxides no separate $3 \mathrm{~d}$-related features are observed [421].

Bismuth vanadate, $\mathrm{BiVO}_{4}$, is used as an anode in photoelectrochemical wa- 
ter splitting. The oxygen $\mathrm{K}$ edge of $\mathrm{BiVO}_{4}$ is different from that of $\mathrm{V}_{2} \mathrm{O}_{5}$ [422]. Mixed oxides with other transition metal ions include the $(\mathrm{V}, \mathrm{Cr}) \mathrm{O}_{2}$ systems [423].

For a full overview of all systems, we refer to the XASEELS database, where all oxygen K edge spectra are collected [424].

\subsubsection{Chromium oxides}

Chromium exists in a large number of valences, from $3 \mathrm{~d}^{4} 2+$ to $3 \mathrm{~d}^{0} 6+$. In 1971 Fischer measured the oxygen $\mathrm{K}$ edge spectra of $\mathrm{Cr}_{2} \mathrm{O}_{3}, \mathrm{CrO}_{3}, \mathrm{Na}_{2} \mathrm{CrO}_{4}$ and $\mathrm{K}_{2} \mathrm{Cr}_{2} \mathrm{O}_{7}$ using a laboratory $\mathrm{x}$-ray source [57]. We compare them below, system by system, with more recent data.

The divalent binary oxide $\mathrm{CrO}$ does not exist as bulk material. It has been made as a thin film and its $\mathrm{Cr} \mathrm{L}_{2,3}$ XAS spectrum has been measured but not its oxygen $\mathrm{K}$ edge spectrum [425]. $\mathrm{Cr}_{2} \mathrm{O}_{3}$ has a $3 \mathrm{~d}^{3}$ ground state forming an

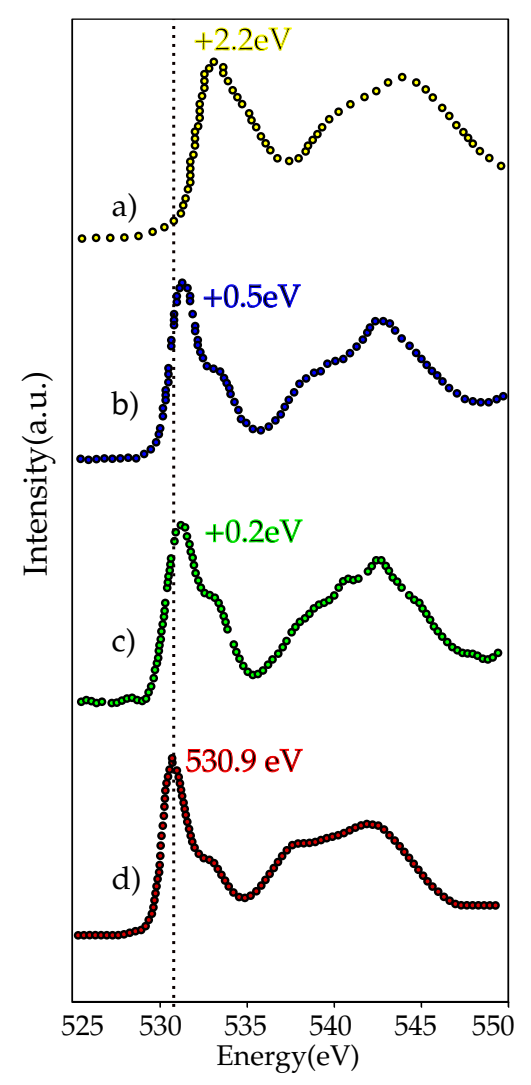

Figure 59: Oxygen $\mathrm{K}$ edge of $\mathrm{Cr}_{2} \mathrm{O}_{3}$. a)[426], b)[389], c)[427], d)[428]. There is variation visible in the spectral shape and especially in the energy calibration.

anti-ferromagnetic ground state. For every $\mathrm{Cr}^{3+}$ ion the $\mathrm{t}_{2 \mathrm{~g}}$ shell is half-filled. The crystal field splitting is equivalent to the exchange splitting yielding a two-peak $3 \mathrm{~d}$-part of the spectrum with the first peak being a combination of 
spin-up $\mathrm{e}_{\mathrm{g}}$ and spin-down $\mathrm{t}_{2 \mathrm{~g}}$ states. The oxygen $\mathrm{K}$ edge of $\mathrm{Cr}_{2} \mathrm{O}_{3}$ has been published in a number of papers $[57,337,389,401,404,426-428]$.

Figure 59 reproduces four published $\mathrm{Cr}_{2} \mathrm{O}_{3}$ spectra. Qualitatively the spectral shapes look equivalent, and some variation is caused by the different experimental resolutions. The second peak/shoulder of the $3 \mathrm{~d}$-part of the spectrum shows intensity variations and the energy calibration of the $\mathrm{Cr}_{2} \mathrm{O}_{3}$ spectrum is different in every publication, depending on the specific calibration method used.

Docherty measured a series of spinel spectra, including $\mathrm{MgCr}_{2} \mathrm{O}_{4}$ and $\mathrm{ZnCr}_{2} \mathrm{O}_{4}$, that all contain $\mathrm{Cr}^{3+}$. The $3 \mathrm{~d}$ part of the measured spectra looks essentially like $\mathrm{Cr}_{2} \mathrm{O}_{3}$ [429]. At higher energies the peaks resemble the delocalized electrons of the spinel crystal structure. Remarkably $\mathrm{LaCrO}_{3}$, containing $\mathrm{Cr}^{3+}$, $3 \mathrm{~d}^{3}$, looks completely different from $\mathrm{Cr}_{2} \mathrm{O}_{3}$ [385]. From local considerations one would expect every, distorted, octahedral $\mathrm{Cr}^{3+}$ to look similar with the two peaks as observed for $\mathrm{Cr}_{2} \mathrm{O}_{3}$. We do not have an explanation for the $\mathrm{LaCrO}_{3}$ spectrum. The $10 \%$ Sr-doped $\mathrm{LaCrO}_{3}$ spectrum measured by Sarma et al. [430] is different from $\mathrm{Cr}_{2} \mathrm{O}_{3}$, where we note that the two published $\mathrm{LaCrO}_{3}$ oxygen $\mathrm{K}$ edges are different from each other.

$\mathrm{CrO}_{2}$ has a rutile structure where $\mathrm{Cr}^{4+}$ occupy a distorted octahedon and has a $3 d^{2}$ ground state with a partly filled spin-up $t_{2 g}$ shell, making it a halfmetallic ferromagnet. The oxygen $\mathrm{K}$ edge of $\mathrm{CrO}_{2}$ has been measured by several groups $[413,426,427,431]$. Goering et al. [431] compared the XMCD spectrum with the spin-polarised DOS. A similar calculation was performed by Kanchana [392]. Both calculations simulate the oxygen is XAS accurately, but fail to simulate the $\mathrm{Cr}_{2,3} \mathrm{XMCD}$, due to their large multiplet effects. Koide et al calculated the angular dependent XMCD and they find that it is important to include spin-orbit coupling [432], i.e. the spin-orbit coupling on the nearest neighbour $\mathrm{Cr}$ atoms is the main contributor to the oxygen $\mathrm{K}$ edge MCD spectral shape. Huang et al. [413] measured the linear dichroism of $\mathrm{CrO}_{2}$ on $\mathrm{TiO}_{2}(100)$. They showed that the first peak is the almost purely the $t_{2 g}$ spin-up state, confirming the half-metallic ferromagnetic state.

$\mathrm{CrO}_{3}$ has a $3 \mathrm{~d}^{0}$ ground state; it is a strong oxidiser and it is known for its carcinogenic properties. The high valence of chromium shifts the oxygen $\mathrm{K}$ edge to lower excitation energy by $3 \mathrm{eV}$ with respect to $\mathrm{Cr}_{2} \mathrm{O}_{3}[57,428]$. The tetrahedral $3 \mathrm{~d}^{0}$ ion $\mathrm{Cr}^{6+}$ complex is measured as $\mathrm{PbCrO}_{4}$ [397] and as $\mathrm{Na}_{2} \mathrm{CrO}_{4}$ [398] which both give similar spectral shapes. This is an important reference material for the study of $\mathrm{Cr}^{6+}$-based paintings [433]. Oxyanions can undergo condensation reaction, as it is the case for chromate ions forming dichromate ions. The binuclear $\left[\mathrm{Cr}_{2} \mathrm{O}_{7}\right]^{2-}$ complex has been measured 
by Diaz et al. [427] and the spectrum is compared to the spectrum of the simple oxyanion in Figure 57. The shape is strongly altered with the broadening and shift to lower energies of the two first peaks. This can be related to the strong distortion of the $\mathrm{CrO}_{4}$ tetrahedra in the dichromate species and increase of three of the four $\mathrm{Cr}-\mathrm{O}$ bond lengths should explain the decrease of the ligand field.

Applications of chromium include catalysts, paints and steel compositions. $\mathrm{CrO}_{2}$ and $\mathrm{Cr}_{2} \mathrm{O}_{3}$ supported on titania are used for the selective catalytic reduction of nitric oxide by ammonia. The shape of oxygen $\mathrm{K}$ edge of $\mathrm{CrO}_{2}$ and $\mathrm{Cr}_{2} \mathrm{O}_{3}$ films on $\mathrm{TiO}_{2}$ were compared with bulk references, suggesting new chromium-oxide phases are not present in the bulk [426]. Lopez et al. studied a series of stainless steels, comparing their chromium, iron and nickel $\mathrm{L}_{2,3}$ edges and oxygen $\mathrm{K}$ edges. The spectra confirmed that in all cases the passive film was formed by $\mathrm{Cr}_{2} \mathrm{O}_{3}[434,435]$.

\subsubsection{Manganese oxides}

Manganese exists in a large number of valences, from $3 \mathrm{~d}^{5} 2+$ to $3 \mathrm{~d}^{0} 7+\mathrm{MnO}$ has a $3 \mathrm{~d}^{5}$ ground state with the spin-up $t_{2 g}$ and $e_{g}$ states occupied.

The oxygen $\mathrm{K}$ edge of $\mathrm{MnO}$ has been published in a number of papers [363, $387,390,436-442]$. MnO is sensitive to the x-ray and electron beams of the XAS and EELS measurements and also $\mathrm{MnO}$ is sometimes modified by defects; some $\mathrm{MnO}$ samples are deliberately doped with defects, for example the $\mathrm{MnO}(1 \% \mathrm{Li})$ sample [437].

The paper of Gilbert et al. [390] studied the sample damage effects and the corrected XAS spectrum is equivalent to the EELS spectrum of Kurata et al. [438]. In first approximation the oxygen K edge will show two peaks respectively related to the empty $t_{2 g}$ and the empty $e_{g}$ states. As discussed in the introduction of the $3 \mathrm{~d}$ oxides, the intensity of the two peaks is expected to be $3: 4$.

What is special in $\mathrm{MnO}$ is that the excitation energy is high due to the high ionicity of $\mathrm{MnO}$, i.e. a large charge transfer energy $\Delta$. In addition $\mathrm{MnO}$ is a rock salt octahedral system that yields a sharp first peak at $539 \mathrm{eV}$ due to the empty manganese $4 \mathrm{sp}$ states. The combination of the two effects means that the $3 \mathrm{~d}$-part of the spectrum is not well separated from the $4 \mathrm{sp}$ part of the spectrum.

$\mathrm{Mn}_{2} \mathrm{O}_{3}$ has a $3 \mathrm{~d}^{4}$ anti-ferromagnetic ground state. For every $\mathrm{Mn}^{3+}$ ion the spin-up $t_{2 g}$ states are occupied. The $e_{g}$ state is half occupied creating a JahnTeller ground state implying significant site distortions. The oxygen $\mathrm{K}$ edge 
of $\mathrm{Mn}_{2} \mathrm{O}_{3}$ has been published in a number of papers [390, 436, 438, 440, 441]. The oxygen $\mathrm{K}$ edge has three peaks that can be assigned respectively to $\mathrm{e}_{\mathrm{g}}$-up, $\mathrm{t}_{2 \mathrm{~g}}$-down and $\mathrm{e}_{\mathrm{g}}$-down. $\mathrm{Mn}_{3} \mathrm{O}_{4}$ has two octahedral $\mathrm{Mn}^{3+}$ sites and one tetrahedral $\mathrm{Mn}^{2+}$ site. The oxygen $\mathrm{K}$ edge can in first approximation be described as the $\mathrm{Mn}_{2} \mathrm{O}_{3}$ spectrum where the second peak is related to the tetrahedral $\mathrm{Mn}^{2+}$ site. We note that there is some variation in the observed spectral shapes of $\mathrm{Mn}_{3} \mathrm{O}_{4}[390,436,438,442]$.

$\mathrm{MnO}_{2}$ has a rutile structure where $\mathrm{Mn}^{4+}$ occupies distorted octahedral sites and has a $3 \mathrm{~d}^{3}$ anti-ferromagnetic ground state. For every $\mathrm{Mn}^{4+}$ ion the spinup $t_{2 g}$ states are occupied. The $\mathrm{MnO}_{2}$ spectrum has two peaks related to the $3 \mathrm{~d}$ part of the spectrum $[337,387,390,436,440-443]$. The first peak is a combination of the spin-up $e_{g}$-states and the spin-down $t_{2 g}$-states. The second peak is related to the spin down $\mathrm{e}_{\mathrm{g}}$-states.

The oxygen $\mathrm{K}$ edge spectra of $\mathrm{MnO}, \mathrm{Mn}_{3} \mathrm{O}_{4} \mathrm{Mn}_{2} \mathrm{O}_{3}$ and $\mathrm{MnO}_{2}$ have been calculated with $\mathrm{FEFF}_{7}$, yielding not very accurate simulations of the general shape [365]. More accurate FEFF calculations were performed by Gilbert et al. [390]. They also remeasured the manganese oxides and carefully corrected for beam damage effects. The calculations essentially confirmed the exchange plus crystal field picture for the $3 \mathrm{~d}$-part of the spectrum and further explain the higher energy spectral shape. The tetrahedral $\mathrm{Mn}^{7+}$ permanganate $\mathrm{MnO}_{4}^{-}$ion has a $3 \mathrm{~d}^{0}$ ground state and has been measured as $\mathrm{KMnO}_{4}[390,398]$. Kurata et al. have measured the $3 \mathrm{~d}^{1}$ equivalent $\mathrm{BaMnO}_{4}$ [438]. However, the spectra shape is very close to $\mathrm{MnO}_{2}$ and although the authors did not mention it, it is possible that the data correspond to a photo-reduced compound.

Minasian et al. [398] measured the oxygen $\mathrm{K}$ edge spectrum of $\mathrm{KMnO}_{4}$ with three different detection methods, respectively transmission XAS in a STXM, TFY XAS and IXS and compared the calculations with TD-DFT calculations. They compared a series of $\mathrm{MO}_{4}^{x-}$ anions and performed a detailed analysis on the trends in their chemical bonding. Suntivich et al. performed a detailed analysis of the chemical bonding of $\mathrm{LaTMO}_{3}$ systems based on the oxygen $\mathrm{K}$ edge [385], for the transition metals $\mathrm{Cr}, \mathrm{Mn}, \mathrm{Fe}, \mathrm{Co}$ and $\mathrm{Ni}$.

The $\mathrm{LaMnO}_{3}$ contains the $3 \mathrm{~d}^{4} \mathrm{Mn}^{3+}$ cation. The oxygen $\mathrm{K}$ edge spectrum has three distinct features, related mainly to respectively the manganese $3 \mathrm{~d}$ states, the lanthanum $5 \mathrm{~d}$ states and the manganese $4 \mathrm{sp}$ states. Equivalent oxygen $\mathrm{K}$ edge of $\mathrm{LaMnO}_{3}$ has also been measured by Abbate et al. [444], Lafuerza et al. [445] and the $\mathrm{PrMnO}_{3}$ by Toulemonde et al. [446]. The $(\mathrm{La}, \mathrm{Sr}) \mathrm{MnO}_{3}$ perovskites show a transition from $\mathrm{Mn}^{3+}$ in $\mathrm{LaMnO}_{3}$ to $\mathrm{Mn}^{4+}$ in $\mathrm{SrMnO}_{3}$ [444]. Changing lanthanum for Sr, replaces the lanthanum 5d 


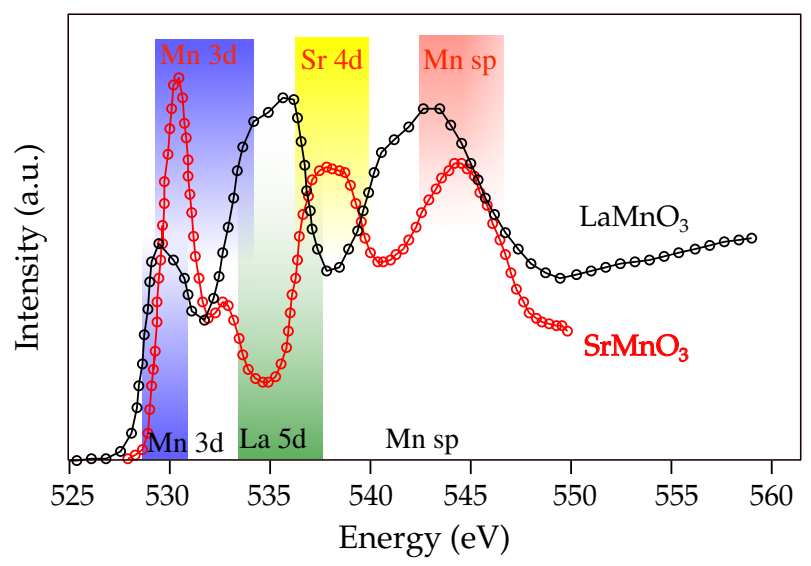

Figure 6o: Oxygen Kedge of $\mathrm{LaMnO}_{3}[385]$ and $\mathrm{SrMnO}_{3}$ [444].The peaks are caused by the Mn $3 \mathrm{~d}$ states, the $\mathrm{La}$ and $\mathrm{Sr} 5 \mathrm{~d} / 4 \mathrm{~d}$ states and the Mn $4 \mathrm{sp}$ states, as indicated.

states for the strontium $4 \mathrm{~d}$ states, while the manganese $3 \mathrm{~d}$ states change from $\mathrm{Mn}^{3+}{ }_{3} \mathrm{~d}^{4}$ to $\mathrm{Mn}^{4+} 3 \mathrm{~d}^{3}$, thereby increasing the intensity of the first structure in the oxygen $\mathrm{K}$ edge.

Lafuerza et al. [445] studied the doping of the manganese sites in $\mathrm{LaMnO}_{3}$ with gallium. The $\mathrm{La}(\mathrm{Mn}, \mathrm{Ga}) \mathrm{O}_{3}$ system shows in first approximation a gradual decrease of the $\mathrm{Mn}^{3+}$ signal, where both features in the $3 \mathrm{~d}$-band decrease and the manganese $4 \mathrm{p}$ band is replaced by a gallium band. The (Pr,Ca) $\mathrm{MnO}_{3}$ system studied by Toulemonde et al. [446], shows a similar change as the $(\mathrm{La}, \mathrm{Sr}) \mathrm{MnO}_{3}$ perovskites; the $3 \mathrm{~d}$-part of the $\mathrm{PrMnO}_{3}$ spectrum is similar to $\mathrm{LaMnO}_{3}$ and $\mathrm{CaMnO}_{3}$ similar to $\mathrm{SrMnO}_{3}$.

Galdi et al. performed a detailed oxygen $\mathrm{K}$ edge study of $\mathrm{LaMnO}_{3} / \mathrm{SrMnO}_{3}$ multilayers [447]. Their spectra for superlattices $\left(\mathrm{SrMnO}_{3}\right)_{n} /\left(\mathrm{LaMnO}_{3}\right)_{2 n}$ mainly showed the lanthanum $5 \mathrm{~d}$ states and no strontium $4 \mathrm{~d}$ states.

Figure 61 compares the oxygen $\mathrm{K}$ edge XAS spectra of $\mathrm{MnO}$ with a series of $(\mathrm{Li}, \mathrm{Mn}) \mathrm{O}_{x}$ oxides, respectively $3 \mathrm{~d}^{4} \mathrm{Li}_{2} \mathrm{MnO}_{3}$, mixed-valent $3 \mathrm{~d}^{3} / 3 \mathrm{~d}^{4}$ $\mathrm{LiMn}_{2} \mathrm{O}_{4}$ and $3 \mathrm{~d}^{3} \mathrm{LiMnO}_{2}$ [437]. The $\mathrm{Li}_{2} \mathrm{MnO}_{3}$ spectrum is similar to that of $\mathrm{MnO}_{2}$ and also the spectrum of the two $3 \mathrm{~d}^{4}$ systems $\mathrm{Mn}_{2} \mathrm{O}_{3}$ and $\mathrm{LiMnO}_{2}$ are similar. 


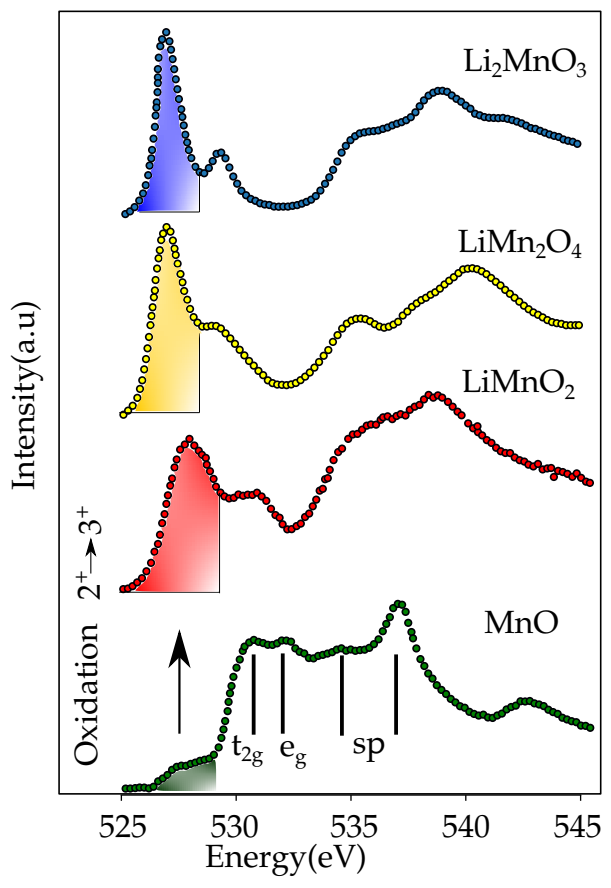

Figure 61: Oxygen $\mathrm{K}$ edge of $\mathrm{MnO}, \mathrm{LiMnO}_{2}, \mathrm{LiMn}_{2} \mathrm{O}_{4}, \mathrm{Li}_{2} \mathrm{MnO}_{3}$. The $3 \mathrm{~d}^{5}$ oxide $\mathrm{MnO}$ contains impurities from $\mathrm{Mn}^{3+}$ as visibly the intensity before 529 $\mathrm{eV}$. The $3 \mathrm{~d}^{4}$ oxide $\mathrm{LiMnO}_{2}$ has a broad $3 \mathrm{~d}$-part due to a combination of the octahedral crystal field, exchange and the Jahn-Teller distortion. The $3 \mathrm{~d}^{3}$ oxide $\mathrm{Li}_{2} \mathrm{MnO}_{3}$ has a first peak related to a combination of spin-up $\mathrm{e}_{\mathrm{g}}$ and spin-down $\mathrm{t}_{2 \mathrm{~g}}$ states. $\mathrm{LiMn}_{2} \mathrm{O}_{4}$ is a mixed valence $\mathrm{Mn}^{3+} / \mathrm{Mn}^{4+}$ oxide and in first approximation its $3 \mathrm{~d}$-part is a combination of the spectra of $\mathrm{Mn}^{3+}$ and $\mathrm{Mn}^{4+}$ sites. 


\subsubsection{Iron oxides}

Iron oxides are among the most abundant materials on earth, in particular $\mathrm{Fe}_{2} \mathrm{O}_{3}$ and $\mathrm{Fe}_{3} \mathrm{O}_{4}$. Iron oxides have valences $3 \mathrm{~d}^{6} 2+$ and $3 \mathrm{~d}^{5} 3^{+} . \mathrm{Fe}^{4+}$ and higher valences are uncommon for iron oxide materials. FeO has a $3 \mathrm{~d}^{6}$ ground state; it is known to be intrinsically unstable regarding its stoichiometry.

The oxygen $\mathrm{K}$ edge of $\mathrm{FeO}$ has been often measured since the first measurement by Grunes in 1982 [92, 363, 388, 401, 404]. All spectra show a first peak related to the iron $3 \mathrm{~d}$-states, that depending on the experimental resolution has some structure. $\mathrm{Fe}_{2} \mathrm{O}_{3}$ has a $3 \mathrm{~d}^{5}$ ground state and appears in several crystal structures, the most common being the corundum $\alpha \mathrm{Fe}_{2} \mathrm{O}_{3}$ structure $[92,337,388,389,404,408,436,448] . \mathrm{Fe}_{2} \mathrm{O}_{3}$ is a half-filled anti-ferromagnet and the first two peaks directly relate to respectively the spin-down $t_{2 g}$ and $\mathrm{e}_{\mathrm{g}}$-states. The oxygen $\mathrm{K}$ edge of $\gamma \mathrm{Fe}_{2} \mathrm{O}_{3}$ looks similar, with some differences at higher energy due to the different crystal structure [92].

The $(\mathrm{La}, \mathrm{Sr}) \mathrm{FeO}_{3}$ perovskites show a transition from $\mathrm{Fe}^{3+}$ in $\mathrm{LaFeO}_{3}$ to $\mathrm{Fe}^{4+}$

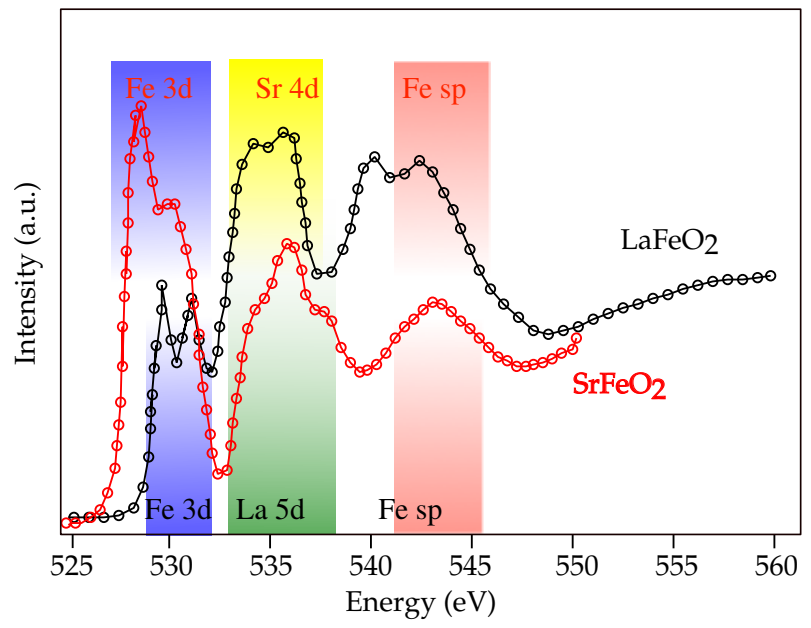

Figure 62: Oxygen $\mathrm{K}$ edge of $\mathrm{LaFeO}_{3}[385]$ and $\mathrm{SrFeO}_{3}[444]$ perovskite. The peaks are respectively caused by the iron $3 \mathrm{~d}$ states, the La and $\mathrm{Sr} 5 \mathrm{~d} / 4 \mathrm{~d}$ states and the iron $4 \mathrm{sp}$ states, as indicated.

in $\mathrm{SrFeO}_{3}$ [444]. The oxygen $\mathrm{K}$ edge of $\mathrm{LaFeO}_{3}$ is compared to $\mathrm{SrFeO}_{3}$ in Figure 62. We observed the $t_{2 g}$ and the $e_{g}$-peak, followed by the lanthanum $5 \mathrm{~d}$ states $[385,449]$. The change from $\mathrm{Fe}^{3+}$ to $\mathrm{Fe}^{4+}$ is osberved in the increase 
of the first feature intensity. The spectrum of $\mathrm{Wu}$ et al. looks similar but the $\mathrm{e}_{\mathrm{g}}$ peak has lower intensity [450]. All formally $\mathrm{Fe}^{4+}$ spectra, $\mathrm{SrFeO}_{3}$ [444, 451] and $\mathrm{BaFeO}_{3}$ [451] look different from each other. The polarization dependent oxygen $\mathrm{K}$ edges of thin films $\mathrm{BiFe}_{3}$ have been studied by Zhenget al. [452].

\subsubsection{Cobalt oxides}

$\mathrm{CoO}$ has a $3 \mathrm{~d}^{7}$ ground state that is always high-spin in oxides. Octahedral $\mathrm{Co}^{2+}$ has a ${ }^{4} \mathrm{~T}_{1 \mathrm{~g}}$ ground state that is split by the $3 \mathrm{~d}$ spin-orbit coupling. The oxygen $\mathrm{K}$ edge of $\mathrm{CoO}$ has two peaks split by the crystal field, respectively related to the empty $t_{2 g}$ state and the empty $e_{g}$ states [389, 439, 448, 449, 453-455]. At higher energy is the typical double structure of the rocksalt crystal structure.

$\mathrm{Co}^{3+}$ has a $3 \mathrm{~d}^{6}$ ground state; in oxides the crystal field is similar in magnitude to the exchange splitting, implying that $\mathrm{Co}^{3+}$ occurs as $\left(\mathrm{t}_{2 \mathrm{~g}}^{6}\right)$ low-spin and as $\left(\mathrm{t}_{2 \mathrm{~g}}^{4} \mathrm{e}_{\mathrm{g}}^{2}\right)$ high-spin oxides. The oxygen $\mathrm{K}$ edge of $\mathrm{Co}_{2} \mathrm{O}_{3}$ has a sharp single peak at the edge indicated a low-spin $\mathrm{Co}^{3+}$ ground state [391]. $\mathrm{Co}_{3} \mathrm{O}_{4}$ is a spinel with tetrahedral $\mathrm{Co}^{2+}$ and octahedral $\mathrm{Co}^{3+}$ sites [453] Because the $\mathrm{Co}^{3+}$ site is more covalent it has more oxygen character and the oxygen $\mathrm{K}$ edge of $\mathrm{Co}_{3} \mathrm{O}_{4}$ is dominated by the low-spin $\mathrm{Co}^{3+}$ features. The peaks of the tetrahedral $\mathrm{Co}^{2+}$ site are superimposed.

The oxygen $\mathrm{K}$ edge of lithium doped $\mathrm{CoO}$ and its end member $\mathrm{LiCoO}_{2}$ have been often studied in relation to charge doping in $\mathrm{CoO}$ and in particular related to battery research $[453,454,456-458] . \mathrm{LiCoO}_{2}$ is a low-spin system with a similar spectral shape as $\mathrm{Co}_{2} \mathrm{O}_{3}$, i.e. a sharp peak related to the empty $\mathrm{e}_{\mathrm{g}}$ states, followed by the cobalt $4 \mathrm{sp}$-band. Doping $\mathrm{CoO}$ with lithium gives oxygen $\mathrm{K}$ edges that can be approximated as a mixture of $\mathrm{CoO}$ and $\mathrm{LiCoO}_{2}$ [453]. In the prevoskite $\mathrm{LaCoO}_{3}$, the $3 \mathrm{~d}^{6} \mathrm{Co}^{3+}$ undergoes a spin transition from low-spin at low temperature to mainly high-spin above $500 \mathrm{~K}$. The $\mathrm{t}_{2 \mathrm{~g}}^{6}$ low-spin system $\mathrm{EuCoO}_{3}$ has the 3 d-peak related to the empty $\mathrm{e}_{\mathrm{g}}$ states at $529.5 \mathrm{eV}$ with a tail to higher energy [459]. The $\mathrm{t}_{2 \mathrm{~g}}^{4} \mathrm{e}_{\mathrm{g}}^{2}$ high-spin system $\mathrm{Sr}_{2} \mathrm{CoO}_{3} \mathrm{Cl}$ contains $\mathrm{CoO}_{5}$ pyramids and has its first $t_{2 \mathrm{~g}}$ peak at $528.5 \mathrm{eV}$ and a second smaller $\mathrm{e}_{\mathrm{g}}$ peak at $530.5 \mathrm{eV}$ [459].

The phase transition in $\mathrm{LaCoO}_{3}$ was studied by Abbate et al. [460]. The transition from the low-spin one-peak spectrum to a broader spectrum is observed, but there is little peak shift and the spectral change is not very clear.

Similar $\mathrm{LaCoO}_{3}$ spectra have been measured $[385,455]$, but as these data 
are obtained at room temperature they are a mixture between low-spin and high-spin. Simbock and coworkers studied the co-existence of low-spin and high-spin ions in $\mathrm{LaCoO}_{3}$ systems of a series of catalytically active systems. They correlate the oxygen $\mathrm{K}$ edge derived electronic parameters with reaction rates $[461]$.

The low-spin $\left(t_{2 g}^{5}\right) \mathrm{Co}^{4+}$ system $\mathrm{SrCoO}_{3}$ shifts to lower energy with respect to low-spin $\mathrm{Co}^{3+}$ oxides and a small shoulder is visible at low energy related to the empty $t_{2 g}$ state [455]. The $(\mathrm{La}, \mathrm{Sr})(\mathrm{Li}, \mathrm{Co}) \mathrm{O}_{3}$ system studied by $\mathrm{Hu}$ et al. contains $40 \% \mathrm{Co}^{4+}$ and the spectral shape is a combination of the low-spin $\mathrm{Co}^{3+}$ and $\mathrm{Co}^{4+}$ peaks $[449,455]$.

Harvey et al. also measured the $\mathrm{Co}^{4+}$ oxides $\mathrm{SrCoO}_{3}$ and $\mathrm{BaCoO}_{3}$ [451], but their observed spectral shapes look different, with significant $\mathrm{Co}^{3+}$ contribution, in addition to large carbonate peaks that make the spectrum complex to analyse above $532 \mathrm{eV}$. The ( $\mathrm{La}, \mathrm{Sr}) \mathrm{CoO}_{3}$ system was studied by Moodenbaugh et al. and by $\mathrm{Hu}$ et al. [449, 462] They concluded that already at $10 \%$ strontium doping the $\mathrm{Co}^{3+}$ switches to high-spin, modifying the whole spectral shape.

The (partial) change in spin state of $\mathrm{Co}^{3+}$ and $\mathrm{Co}^{4+}$ sites makes the comparison of the oxygen $\mathrm{K}$ edge spectra of $\mathrm{Co}^{3+}$ and $\mathrm{Co}^{4+}$ systems very complex to analyse on their own, without other information such as the cobalt $\mathrm{L}_{2,3}$ edge.

\subsubsection{Nickel oxides}

$\mathrm{NiO}$ has a $3 \mathrm{~d}^{8}$ ground state with two $\mathrm{e}_{\mathrm{g}}$ holes. The oxygen $\mathrm{K}$ edge of $\mathrm{NiO}$ is one of the most published oxygen $\mathrm{K}$ edge spectra, where in this review we limit the citations to the papers that focus on the spectral shape $[337,363$, $389,401,404,439,455,463-468]$. A full overview of all published $\mathrm{NiO}$ spectra is given in the XASEELS database [424]. The NiO spectrum has been measured over a long energy range by Zschech et al. [465], where they focused on the self-absorption effects in FY detection. We refer to the experimental section for further discussion of FY detection.

The $3 \mathrm{~d}^{7} \mathrm{Ni}^{3+}$ system $\mathrm{Nd}_{2} \mathrm{Li}_{0.5} \mathrm{Ni}_{0.5} \mathrm{O}_{4}$ is considered to be a low-spin system based on its $\mathrm{Ni} \mathrm{L}_{2,3}$ edge. The oxygen $\mathrm{K}$ edge shows a shift of $2.6 \mathrm{eV}$ with respect to $\mathrm{NiO}$ with a sharp peak at the edge related to the three empty $\mathrm{e}_{\mathrm{g}}$-states $[455,467,469]$.

Suntivich et al. measured $\mathrm{LaNiO}_{3}$ and a series of rare-earth (RE) $\mathrm{RENiO}_{3}$ oxides [385]. The oxygen $\mathrm{K}$ edge looks similar in all $\mathrm{RENiO}_{3}$ oxides and shows an intense sharp peak at the edge similar also to the $\mathrm{Nd}_{2} \mathrm{Li}_{0.5} \mathrm{Ni}_{0.5} \mathrm{O}_{4}$ 
system and suggesting a low-spin state for all systems. This is in contrast to the $\mathrm{Ni} \mathrm{L}_{2,3}$ edge that shows a change from low-spin to high-spin for example in case of $\mathrm{EuNiO}_{3}$ [470]. The layered perovskite $(\mathrm{Nd}, \mathrm{Sr})_{2} \mathrm{NiO}_{4}$ shows a different oxygen $\mathrm{K}$ edge compared with the $\mathrm{Nd}_{2} \mathrm{Li}_{0.5} \mathrm{Ni}_{0.5} \mathrm{O}_{4}$ system, likely due to a partial high-spin ground state [455].

Similar oxygen $\mathrm{K}$ edge spectra are measured for $\mathrm{La}_{2} \mathrm{NiO}_{4}$ containing $3 \mathrm{~d}^{8}$ $\mathrm{Ni}^{2+}[471]$. Hu et al. measured the polarization dependent oxygen $\mathrm{K}$ edge of the $\mathrm{Ni}^{2+}$ oxide $\mathrm{Y}_{2} \mathrm{BaNiO}_{5}$ [472]. Upon doping the yttrium sites with calcium a $\mathrm{Ni}^{3+}$ state is found [472].

\subsubsection{Copper oxides}

$\mathrm{CuO}$ has a $3 \mathrm{~d}^{9}$ ground state with one $\mathrm{e}_{\mathrm{g}}$ hole. $\mathrm{CuO}$ has a rather complex crystal structure with low symmetry. The oxygen $\mathrm{K}$ edge of $\mathrm{CuO}$ has a sharp peak at the edge related to the empty $\mathrm{e}_{\mathrm{g}}$ state $[337,363,401,404,467,473$, 474]. At higher energy the spectrum appears quite different from all other rocksalt divalent transition metal oxides, related to its monoclinic crystal structure. The $\mathrm{CuO}$ spectral shape has been calculated by Jiang et al. [474]. The oxygen $\mathrm{K}$ edge of $\mathrm{Cu}_{2} \mathrm{O}$ was measured by Stohr et al. in 1980 [58]. They measured the spectrum over an energy range of $400 \mathrm{eV}$. Jiang et al. calculated the oxygen $\mathrm{K}$ edge of $\mathrm{Cu}_{2} \mathrm{O}$ in good agreement with experiments [473, 474]. $\mathrm{Hu}$ et al. measured the oxygen $\mathrm{K}$ edge RIXS of $\mathrm{Cu}_{2} \mathrm{O}$ [475]. The oxygen $\mathrm{K}$ edge spectrum of the formally $\mathrm{Cu}^{3+}$ oxide $\mathrm{LaLi}_{0.5} \mathrm{Cu}_{0.5} \mathrm{O}_{3}$ has been studied by $\mathrm{Hu}$ et al. $[467,469]$.

The oxygen K edges measured with XAS and EELS up to 1994 have been reviewed by Fink et al. [476]. The study on the effect of Sr-doping in the layered perovskite $\mathrm{La}_{2} \mathrm{CuO}_{4}$ has been studied with oxygen $\mathrm{K}$ edges using EELS [477] and XAS [478]. $\mathrm{La}_{2} \mathrm{CuO}_{4}$ has a $\mathrm{Cu}^{2+}$ related peak at $530.5 \mathrm{eV}$. Upon doping with $\mathrm{Sr}$, part of the copper is changed to $\mathrm{Cu}^{3+}$ with a peak at $528.5 \mathrm{eV}$, where the $528.5 \mathrm{eV}$ peak shifts slightly down upon increased doping and the $530.5 \mathrm{eV}$ peak shifts slightly up.

Recent contributions on the oxygen K edge of cuprates include RIXS studies [479] and the measurement of layer specific spectra using a STEM EELS study [480].

\subsection{OVERVIEW OF THE 4D AND 5D OXIDES}

The crystal field splitting in $4 \mathrm{~d}$ oxides is larger than for $3 \mathrm{~d}$ oxides, typically around $3.5 \mathrm{eV}$ for octahedral sites. This implies that $4 \mathrm{~d}$ oxides always are in a 


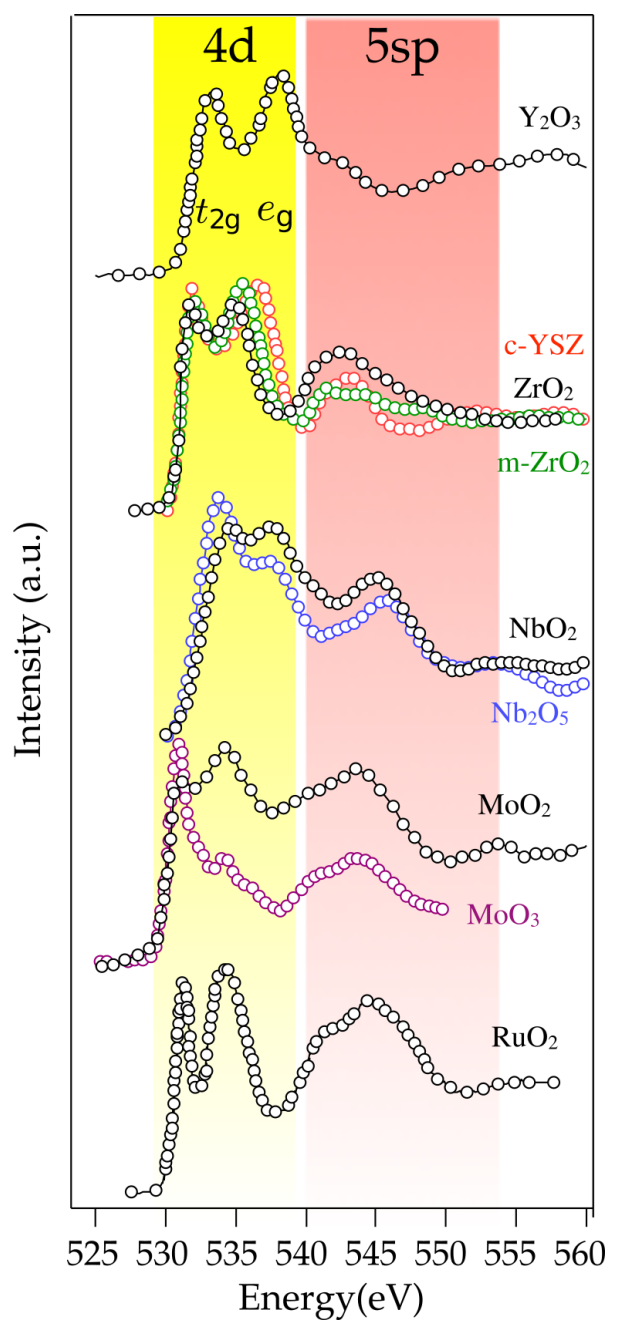

Figure 63: Oxygen $\mathrm{K}$ edge spectra of $\mathrm{Y}_{2} \mathrm{O}_{3}$ [481], tetragonal [482] and monoclinic [483] (m-) $\mathrm{ZrO}_{2}$ and cubic yttrium stabilised zirconia [483] (c-YSZ), $\mathrm{NbO}_{2}$ and $\mathrm{Nb}_{2} \mathrm{O}_{5}$ [484], $\mathrm{MoO}_{3}$ [485] and $\mathrm{MoO}_{2}$ [486] and $\mathrm{RuO}_{2}$ [487].Visible are respectively the $t_{2 g}$ states, the $e_{g}$ states and the empty sp-bands of the metal ion. 
low-spin ground state. Figure 63 shows a selection of oxygen K edge spectra for $4 \mathrm{~d}$ oxides. Most oxides are not magnetic implying that the assignment of the peaks is essentially the same for all $4 \mathrm{~d}$ oxides in octahedral symmetry: respectively (a) the $4 \mathrm{~d}_{2 g}$ states, (b) the $4 \mathrm{~d} \mathrm{e}_{\mathrm{g}}$ states and (c) the empty spbands of the metal ion. Mixed oxides have the empty states of the other cation superimposed.

\subsubsection{4d transition metal oxides}

Yttrium oxides: Yttria, $\mathrm{Y}_{2} \mathrm{O}_{3}$, is a wide-gap semiconductor with a band gap of $5.6 \mathrm{eV}$. It has a cubic crystal structure where $\mathrm{Y}$ cations are 6-fold coordinated in distorted octahedrons. The oxygen $\mathrm{K}$ edge of $\mathrm{Y}_{2} \mathrm{O}_{3}$ (Figure 63) shows two main features corresponding to the crystal field split $4 \mathrm{~d}$ states with the $t_{2 g}$ peak at $533 \mathrm{eV}$ and the $e_{g}$ peak at $538 \mathrm{eV}$, implying a crystal field of $5.0 \mathrm{eV}$. We note that there is an energy spread of $3.0 \mathrm{eV}$ in the energy calibration of the first peak in the publications [481, 483, 488-490]. Similar variations in calibration occur in many other oxygen K edge spectra.

Zirconium oxides: The most common oxide of zirconium is $\mathrm{ZrO}_{2}$, which is also known as zirconia. $\mathrm{ZrO}_{2}$ occurs in three phases, cubic, tetragonal and monoclinic, and one can use an impurity element to stabilize one of those phases.

The oxygen $\mathrm{K}$ edge of $\mathrm{ZrO}_{2}$ (Figure 63) shows subtle differences between the different polymorphs. It mainly contains the $4 \mathrm{~d} \mathrm{t}_{2 \mathrm{~g}}$ peak at $532.4 \mathrm{eV}$ and the $4 \mathrm{~d} \mathrm{e}_{\mathrm{g}}$ peak at $535.5 \mathrm{eV}$, implying a crystal field of $3.1 \mathrm{eV}[482,483$, 491-496]. Several investigations of the oxygen $\mathrm{K}$ edges in $\mathrm{ZrO}_{2}$ and yttrium stabilised zirconia (YSZ) samples with varying compositions have been reported demonstrating that the spectra are very sensitive to local crystalline structure and dopant content. For example ytrria stabilised zirconia $\mathrm{ZrO}_{2}[\mathrm{Y}]$ is an important ceramic material $[483,497]$ used in dental crowns and nonmetal knife blades. Recently a number of other dopants have been studied in relation to the photocatalytic properties of $\mathrm{ZrO}_{2}$, including $\mathrm{ZrO}_{2}[\mathrm{Ce}]$ [494, 496] and $\mathrm{ZrO}_{2}[\mathrm{Cu}]$ [495].

\section{Niobium oxides:}

Niobium has three oxides, respectively divalent $4 \mathrm{~d}^{3}$ in the cubic $\mathrm{NbO}$, tetravalent $4 \mathrm{~d}^{1}$ in the tetragonal $\mathrm{NbO}_{2}$ and pentavalent $4 \mathrm{~d}^{0} \mathrm{Nb}_{2} \mathrm{O}_{5} \cdot \mathrm{Nb}_{2} \mathrm{O}_{5}$ and also $\mathrm{Nb}_{3} \mathrm{O}_{7}(\mathrm{OH})$ nanostructures are promising materials in the field of photoelectrodes in dye-sensitized solar cells and photoelectrochemical cells [498]. 
Olszta et al. compared the oxygen $\mathrm{K}$ edges of the three oxides [484]. Figure 63 shows the spectra of $\mathrm{NbO}_{2}$ and $\mathrm{Nb}_{2} \mathrm{O}_{5}$. The crystal field splitting of the first two peaks corresponding to the $4 \mathrm{~d}$ states is further reduced compared to $\mathrm{Y}$ and $\mathrm{Zr}$ oxides. The first peak of the oxygen $\mathrm{K}$ edges shifts from $533.5 \mathrm{eV}\left(\mathrm{Nb}_{2} \mathrm{O}_{5}\right)$ to $534.3 \mathrm{eV}\left(\mathrm{NbO}_{2}\right)$ and $535.1 \mathrm{eV}(\mathrm{NbO})$, as determined by our measurements on the published spectra. The shifts are inverted at the $\mathrm{Nb} \mathrm{M}_{3}$ edge, i.e. respectively $365.9 \mathrm{eV}\left(\mathrm{Nb}_{2} \mathrm{O}_{5}\right)$ to $364.6 \mathrm{eV}\left(\mathrm{NbO}_{2}\right)$ and 362.8 $\mathrm{eV}(\mathrm{NbO})$. Note that the relative shifts are larger for the $\mathrm{Nb} \mathrm{M}_{3}$ edge than for the oxygen $\mathrm{K}$ edge, related to the larger core hole effect for the $\mathrm{Nb} 3 \mathrm{p}$ core holes. Soriano et al. compared the $\mathrm{Nb}_{2} \mathrm{O}_{5}$ spectrum to the other $3 \mathrm{~d}^{0}$, $4 \mathrm{~d}^{0}$ and $5 \mathrm{~d}^{0}$ oxides [482]. The $5 \mathrm{~d}^{0}$ oxide $\mathrm{KNbO}_{3}$ has its first peak at $531.1 \mathrm{eV}$ [499], at lower energy than $\mathrm{Nb}_{2} \mathrm{O}_{5}$ (531.6 eV [482] resp. 533.3 eV [484]) The crystal field is also $0.5 \mathrm{eV}$ larger for $\mathrm{KNbO}_{3}$.

Molybdenum oxides: Molybdenum has two binary oxides, $4 \mathrm{~d}^{0} \mathrm{MoO}_{3}$ and $4 \mathrm{~d}^{2} \mathrm{MoO}_{2} \cdot \mathrm{MoO}_{2}$ has a distorted rutile structure. The oxygen $\mathrm{K}$ edge of $\mathrm{MoO}_{2}$, shown in Figure 63, has peaks related to the empty $4 \mathrm{~d}$ states, but there is significant variation in the published spectra [486, 500-502]. Thakur et al. compared TEY and TFY spectra with high resolution [501] and they observed 3 peaks within the $4 \mathrm{~d}$-band that they assigned to a distortion of the octahedral site.

The 3 features were also observed by Khyzhun et al. [500]. Wang et al. studied the reduction of $\mathrm{MoO}_{3}$ to $\mathrm{MoO}_{2}$ in the electron beam during an EELS experiment [486]. Possibly all $\mathrm{MoO}_{2}$ spectra contain some admixture of $\mathrm{MoO}_{3}$ but as the peaks essentially overlap it is difficult to determine such mixing quantitatively.

The $4 \mathrm{~d}^{0}$ system $\mathrm{MoO}_{3}$ is used as an oxidation catalyst. Its oxygen $\mathrm{K}$ edge spectrum is compared to $\mathrm{MoO}_{2}$ in Figure 63. Schlogl et al. measured the oxygen $\mathrm{K}$ edge of a single crystal $\mathrm{MoO}_{3}$, which they compared with a partly reduced sample $\mathrm{MoO}_{2.8}$ [503]. The other published $\mathrm{MoO}_{3}$ spectra are all similar to this reduced spectrum $[485,486,502,504] \mathrm{Wu}$ et al. measured their $\mathrm{MoO}_{3}$ sample both with electron yield and with ion yield. In ion yield one detects the $\mathrm{O}^{+}$ions that escape from the sample upon $\mathrm{x}$-ray irradiation. Ion yield is an extremely surface sensitive technique and measures only oxygen atoms in the surface layer of the system [485].

Technetium oxides: Minasian et al. studied a series of $\mathrm{d}^{0}$ tetrahedral oxyanions of $3 \mathrm{~d}, 4 \mathrm{~d}$ and $5 \mathrm{~d}$ metal ions, including the pertechnetate $\mathrm{TcO}_{4}^{-}$ anion [398]. They simulated all oxygen K edge spectra with TD-DFT and ob- 
tained good agreement with the experimental spectra. The first two peaks in the spectra are respectively the transition to the empty $e$ and $t_{2}$ states, split by approximately $2.0 \mathrm{eV}$ in all six systems. The excitation energy of the first peak is lowest in the $3 \mathrm{~d}$ systems, followed by $4 \mathrm{~d}$ and $5 \mathrm{~d}$. In addition the $\mathrm{Mn}, \mathrm{Tc}$ and Re oxyanions have lower excitation energies than their $\mathrm{Cr}$, $\mathrm{Mo}$ and $\mathrm{W}$ analogs. There is a linear relation between the first peak energy position in the oxygen $\mathrm{K}$ edge and the first optical excitation energy, i.e.from the highest MO to the empty e states [398].

Ruthenium oxides: The $4 \mathrm{~d}^{4}$ system $\mathrm{RuO}_{2}$ has a rutile structure. Its oxy-

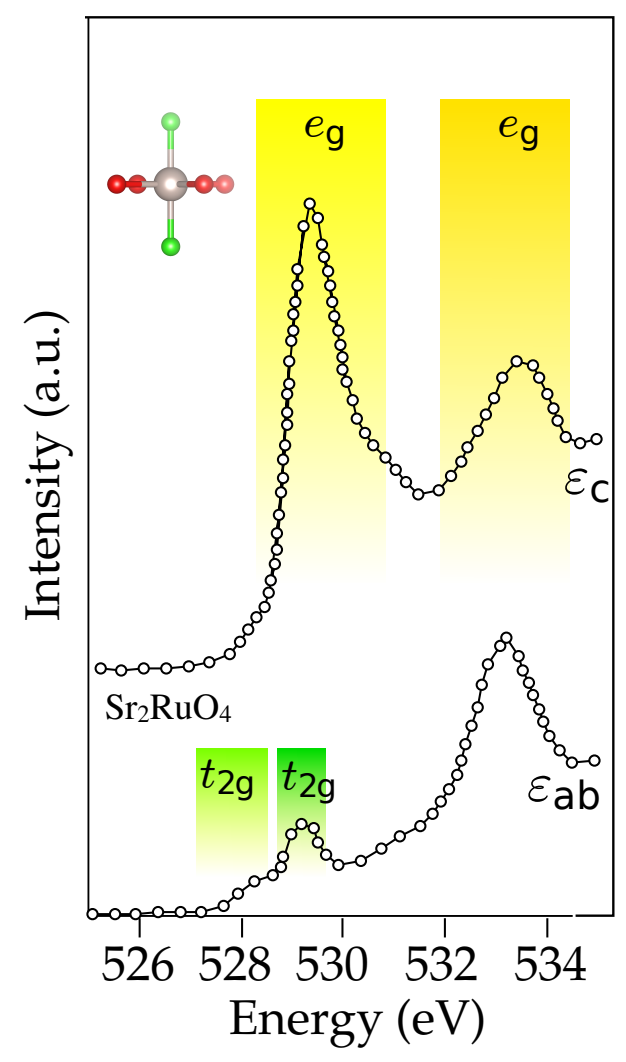

Figure 64: Oxygen $\mathrm{K}$ edge of $\mathrm{Sr}_{2} \mathrm{RuO}_{4}$ layered perovskite[505] with the incident electrical field parallel to the layer $\left(\varepsilon_{\mathrm{ab}}\right)$ and perpendicular to the layer $\left(\varepsilon_{\mathfrak{c}}\right)$. The contributions from the apical and in-plane oxygens are highlighted.

gen K edge, Figure 63 , has a sharp peak related to the $t_{2 g}$ states followed by a broader peak related to the $\mathrm{e}_{\mathrm{g}}$ states $[487,506,507]$. The oxygen $\mathrm{K}$ edge of $\mathrm{RuO}_{2}$ bears much resemblance to the other rutile oxides such as $\mathrm{TiO}_{2}$ and $\mathrm{IrO}_{2}$ (see $5 \mathrm{~d}$ element section).

Tsai et al. studied $\mathrm{RuO}_{2}$ nanorods for field emission applications [487].

Ruthenates are systems that contain the $\mathrm{RuO}_{6}$ octahedrons combined with other cations, mainly $\mathrm{Sr}$ and $\mathrm{Ca}$. The most studied material is $\mathrm{Sr}_{2} \mathrm{RuO}_{4}$ and 
is a layered perovskite with a structure similar to $(\mathrm{La}, \mathrm{Sr})_{2} \mathrm{CuO}_{4}$, which also shares the superconducting properties with the copper analogs, though with critical temperature of less than one Kelvin. In 1996 Schmidt et al. measured the angular dependence of $\mathrm{Sr}_{2} \mathrm{RuO}_{4}$ single crystals [505].

Figure 64 shows the angular dependence of the oxygen $\mathrm{K}$ edge spectrum of $\mathrm{Sr}_{2} \mathrm{RuO}_{4}$. The first two peaks at 528.5 and 529.5 are assigned to empty $4 \mathrm{~d} \mathrm{t}_{2 \mathrm{~g}}$ states, where the first peak is related to the apical oxygen and the second to the in-plane oxygen. The following two peaks at $531 \mathrm{eV}$ and $533.5 \mathrm{eV}$ are related to $4 \mathrm{~d} \mathrm{e}_{\mathrm{g}}$ states and have higher intensity in the out-of-plane direction. This assignment is essentially reproduced in later experiments on $\mathrm{Sr}_{2} \mathrm{RuO}_{4}$ and $\mathrm{Ca}_{2} \mathrm{RuO}_{4}[506,508-511]$.

Fatuzzi et al. performed an elegant experiment to distinguish the three $2 p$ orbitals $\mathrm{x}, \mathrm{y}$ and $\mathrm{z}$, from a combination of angular and polarization dependence. They performed a RIXS experiment on $\mathrm{Sr}_{2} \mathrm{RuO}_{4}$ and $\mathrm{Ca}_{2} \mathrm{RuO}_{4}$ and analyse the low energy excitations as a combination of the crystal field splitting of the $t_{2 g}$ states and the effect of the $4 \mathrm{~d}$ spin-orbit coupling [510]. One can make more complex layered oxides from combinations of $\mathrm{Sr}$ and $\mathrm{Ru}$. Keeping the valence of $\mathrm{Ru}$ at $4^{+}$the series includes several combinations of $\mathrm{SrO}$ with $\mathrm{RuO}_{2}$, i.e $(\mathrm{SrO}) \times\left(\mathrm{RuO}_{2}\right) \mathrm{y}$, including $\mathrm{SrRuO}_{3}, \mathrm{Sr}_{2} \mathrm{RuO}_{4}, \mathrm{Sr}_{3} \mathrm{Ru}_{2} \mathrm{O}_{7}$ and $\mathrm{Sr}_{4} \mathrm{Ru}_{3} \mathrm{O}_{10}$, where a range of magnetic and superconducting properties are found in these systems.

The oxygen $\mathrm{K}$ edges of the $\mathrm{Sr}_{2} \mathrm{RuO}_{4}, \mathrm{Sr}_{3} \mathrm{Ru}_{2} \mathrm{O}_{7}$ and $\mathrm{Sr}_{4} \mathrm{Ru}_{3} \mathrm{O}_{10}$ ruthenates have been measured by Malvestuto et al. [509]. In addition, one can change the valence of $\mathrm{Ru}$ and make the $\mathrm{Ru}^{5+}$ system $\mathrm{Sr}_{4} \mathrm{Ru}_{2} \mathrm{O}_{9}$ for which $\mathrm{Hu}$ et al. measured the oxygen $\mathrm{K}$ edge [506] Chikamatsu et al. compared the $\mathrm{Sr}_{2} \mathrm{RuO}_{4}$ system with the oxyfluoride system $\mathrm{Sr}_{2} \mathrm{RuO}_{3} \mathrm{~F}_{2}$. The oxygen $\mathrm{K}$ edge spectra, in relation to other core and valence band spectroscopies, indicate that the $\mathrm{Ru}^{4+}$ in $\mathrm{Sr}_{2} \mathrm{RuO}_{3} \mathrm{~F}_{2}$ is in a high-spin ground state, in contrast to the low-spin $\mathrm{Sr}_{2} \mathrm{RuO}_{4}$ systems [511].

Palladium oxides: Palladium is an important catalyst, which reactivity is enhanced by fabricating nano sized metallic particles. The oxidation of the surface of the nanoparticles motivates their study with microscopy and spectroscopy.

Sun et al. studied a $\mathrm{Pd} / \mathrm{Al}_{2} \mathrm{O}_{3}$ system [512]. They measured the oxygen $\mathrm{K}$ edge of the $\mathrm{Pd}$ nanoparticles with EELS and observed an oxygen $\mathrm{K}$ edge that is different from the reference PdO. To interpret the experimental data obtained on nanoparticles, the oxygen $\mathrm{K}$ edge of $\mathrm{PdO}$ was calculated for three crystal structures by Mogi et al. [513]. They used Wien2K calculations 
and reproduced the main features of the experimental spectrum.

Silver oxides: Metallic silver is importantly used for its surface reactivity and several studies investigated the absorbed oxygen reactivity on the metal surface, in particular for ethylene epoxidation [514-516]. The oxygen K edge of $\mathrm{Ag}_{2} \mathrm{O}$ shows a first intense peak corresponding the $\mathrm{O}$ 2p states hybridized with the $\mathrm{Ag} 4 \mathrm{~d}$ states followed by the $\mathrm{O} 2 \mathrm{p} / \mathrm{Ag} 5 \mathrm{sp}$ bands. Comparison with absorbed oxygen atoms reveal that the nucleophilic species bind differently from the electrophilic species at the silver surface.

Ruzankin et al. studied the ethylene epoxidation on the silver surface. They observed several features in the oxygen $\mathrm{K}$ edge that were simulated with an $\mathrm{Ag}_{2} \mathrm{O}$ molecule [516]. Another oxygen $\mathrm{K}$ edge spectrum has been published for silver foil that was treated with $\mathrm{C}_{2} \mathrm{H}_{4}$ and $\mathrm{O}_{2}$ at $470 \mathrm{~K}$ [514].

\subsection{2 $5 d$ transition metal oxides}

Hafnium oxides: Hafnium oxide $\mathrm{HfO}_{2}$ is an important material in the semiconductor industry due to its high dielectric constant $\mathrm{k}$. These so-called high

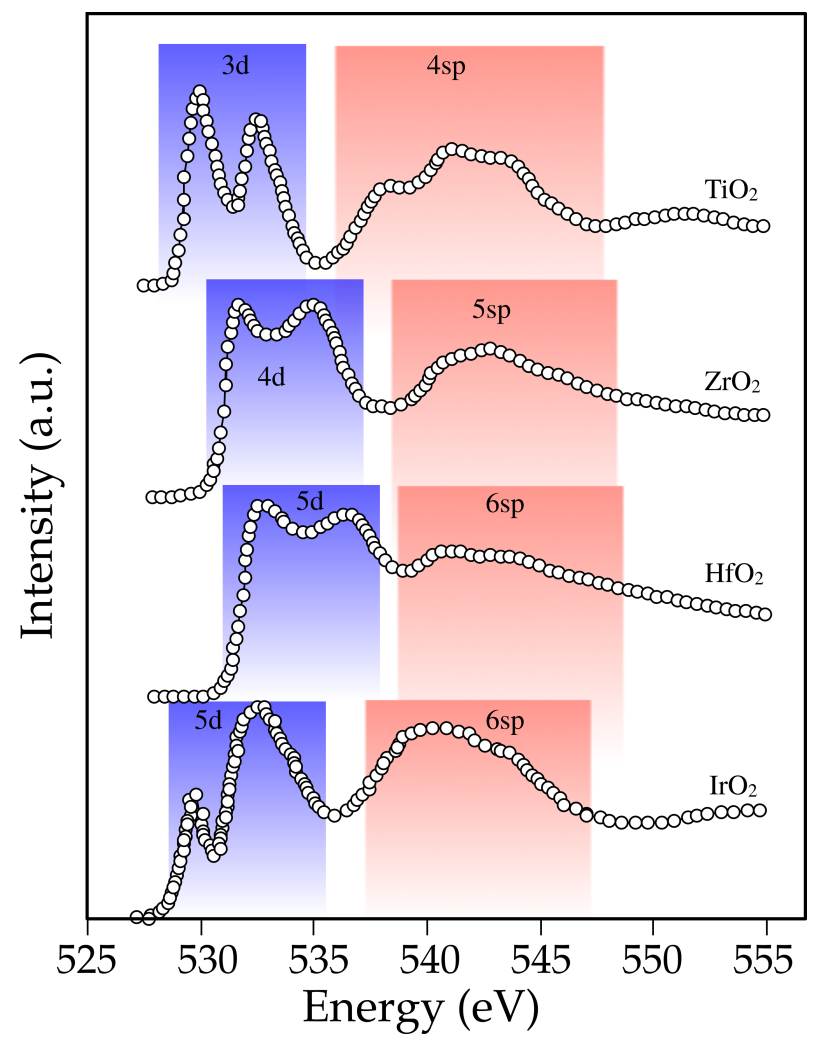

Figure 65: Oxygen $\mathrm{K}$ edge of $\mathrm{HfO}_{2}$ compared to its $3 \mathrm{~d}$ and $4 \mathrm{~d}$ analogs $\mathrm{TiO}_{2}$ (rutile) and $\mathrm{ZrO}_{2}$ [482] and to $\mathrm{IrO} 2$ [487] (rutile). 
$\mathrm{k}$ materials are used to replace a silicon dioxide layer of a device, which is important for further miniaturization of microelectronic components.

Soriano et al. compared $\mathrm{HfO}_{2}$ with its $3 \mathrm{~d}$ and $4 \mathrm{~d}$ analogs $\mathrm{TiO}_{2}$ and $\mathrm{ZrO}_{2}$ [482]. Unlike $\mathrm{TiO}_{2}, \mathrm{HfO}_{2}$ presents the same crystal structures and polymorphs as zirconia. The oxygen $\mathrm{K}$ edge spectrum of $\mathrm{HfO}_{2}$ is shown in Figure 65 along with its analogs from Group IVb. The two first features correspond to the metal $5 \mathrm{~d}$ bands and the higher energy features correspond to the $6 \mathrm{sp}$ states. The crystal field splitting and the total dispersion of the metal $\mathrm{d}$ band increase from $\mathrm{Ti}$ to $\mathrm{Zr}$ to Hf revealing larger interactions caused by larger nd orbitals. The absorption threshold shifts to higher energies in the series in agreement with the larger band gaps for the oxides of the heavier elements. Since 2003 there have been a series of publications that studied the interface of $\mathrm{HfO}_{2}$ with $\mathrm{Si}$ [517-521]. In addition to $\mathrm{HfO}_{2}$ the oxygen $\mathrm{K}$ edge has been measured of amorphous $\mathrm{HfSiO}$ [521] and $\mathrm{HfSiON}$ [522].

Tantalum oxides: The Tantalum oxides $\mathrm{Ta}_{2} \mathrm{O}_{5}$ is an important material in memory resistors in memory devices. Kumar et al. studied the oxygen $\mathrm{K}$ edge spectrum of $\mathrm{Ta}_{2} \mathrm{O}_{5}$ in such devices with a $30 \mathrm{~nm}$ resolved STXM experiment [523]. The oxygen $\mathrm{K}$ edge of the tantalum oxide $\mathrm{Ta}_{2} \mathrm{O}_{5}$ has two peaks at the edge, related to respectively the Ta $5 \mathrm{~d} \mathrm{t}_{2 g}$ and $\mathrm{e}_{g}$ states $[482,523,524]$.

Tungsten oxides: Tungsten oxide $\mathrm{WO}_{3}$ is a $5 \mathrm{~d}^{0} \mathrm{~W}^{6+}$ system that occurs in a number of crystal structures.

Purans et al. studied six different tungsten oxides. Figure 66 shows the spectrum of monoclinic $\mathrm{WO}_{3}$. All spectra have a sharp first peak related to the empty $t_{2 g}$ states. The second structure related to the empty $e_{g}$ states overlaps with the structure related to the tungsten s and p empty states [499, 504, 525]. Oxygen $\mathrm{K}$ edge spectra have been published for films and nanorods [526530] and $\gamma-\mathrm{WO}_{3}$ is doped with $\mathrm{Na}$ to form sodium-tungsten bronze, where the sodium atoms occupy a fraction of the 12-coordinate interstitial A-sites in the $\mathrm{WO}_{6}$ octahedra framework. The oxygen $\mathrm{K}$ edge of $\mathrm{Na}_{x} \mathrm{WO}_{3}$ shows a similar first peak as $\mathrm{WO}_{3}$ related to the $\mathrm{t}_{2 \mathrm{~g}}$ states. The region between $535 \mathrm{eV}$ and $545 \mathrm{eV}$ shows a different spectral shape, which is related to the differences in crystal structure despite the same octahedral environment for $\mathrm{W}$ ions $[504,525,529]$. The $\mathrm{Na}_{2} \mathrm{WO}_{4}$ system has a quite different structure from the bronzes. This system contains tetrahedral complexes of the tungsten oxyanion $\mathrm{WO}_{4}^{2-}$. Its oxygen $\mathrm{K}$ edge has two sharp peaks related to respectively the $t_{2}$ and the e empty states [398]. 

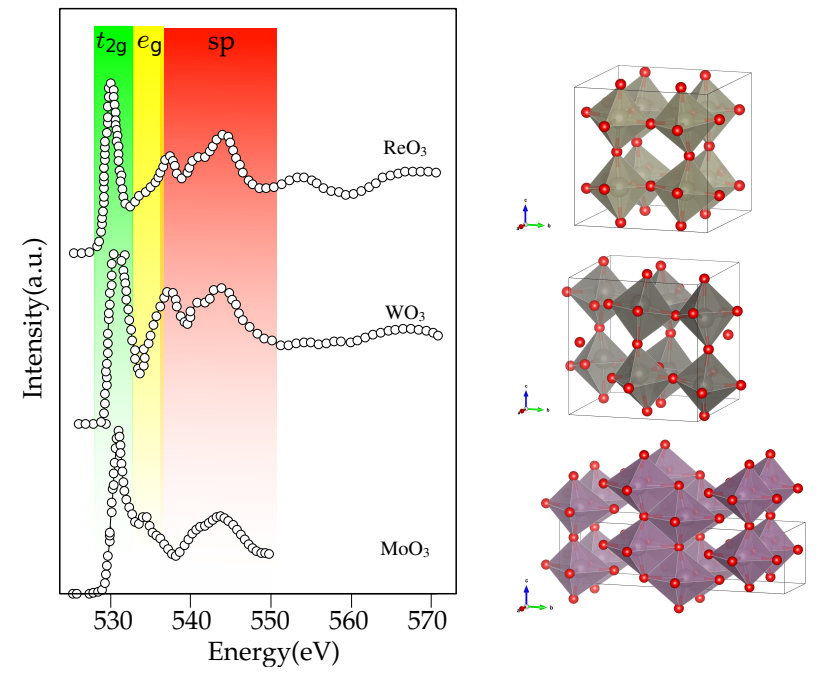

Figure 66: Oxygen $\mathrm{K}$ edge spectra of monoclinic $\mathrm{WO}_{3}$ and cubic $\mathrm{ReO}_{3}$ compared to $\mathrm{MoO}_{3}$ (orthorhombic) with the assignment of the spectral features [504].

Rhenium oxides: Rhenium oxide $\mathrm{ReO}_{3}$ has a similar structure to that of perovskites and contains $\operatorname{Re}^{6+}{ }_{5} \mathrm{~d}^{1}$ ions.

Purans et al. measured its oxygen K edge spectrum [499, 525] and investigated the effect of the distortion of the $\mathrm{ReO}_{6}$ octahedron on the spectrum. The spectrum is shown in Figure 66 together with monoclinic $\mathrm{WO}_{3}$. The $\mathrm{ReO}_{3}$ spectrum bears much resemblance to the $\mathrm{WO}_{3}$ and $\mathrm{MoO}_{3}$ oxygen $\mathrm{K}$ edge. The $\mathrm{Re}^{7+}$ system $\mathrm{NaReO}_{4}$ contains $\mathrm{Re}$ in a tetrahedral surroundings and its oxygen $\mathrm{K}$ edge is similar to that of the other tetrahedral oxyanion complexes [398]. The oxygen $\mathrm{K}$ edge of the series of oxyanion is discussed and compared in the section on $3 \mathrm{~d}$ metal oxides.

Iridium oxides: The iridium oxide $\mathrm{IrO}_{2}$ contains $\mathrm{Ir}^{4+} 5 \mathrm{~d}^{5}$ ions and has a rutile structure. The oxygen $\mathrm{K}$ edge spectrum is shown in Figure 65 where it is compared to $\mathrm{TiO}_{2}$ rutile and $\mathrm{HfO}_{2}$. The shape is very similar to the spectrum of $\mathrm{TiO}_{2}$ revealing the effect of the rutile crystal structure. It contains a small first peak related to the single hole in the $t_{2 g}$ state followed by a large peak related to the $e_{g}$ states $[212,487,504,531]$. Kim et al. measured an epitaxial $\mathrm{IrO}_{2}$ film on $\mathrm{TiO}_{2}$. They observe that the sharp $\mathrm{t}_{2 \mathrm{~g}}$-peak has a very strong angular dependence with almost all intensity being related to the sigma direction. This observation implies that this empty $t_{2 g}$ state lacks involvement of an Ir $\mathrm{d}_{x y}$ orbital, which in turn implies that despite the importance of the $5 \mathrm{~d}$ spin-orbit coupling in $\mathrm{IrO}_{2}$, the $\mathrm{J}_{\text {eff }}=1 / 2$ state (as observed in the iridates, see below) does not form in $\mathrm{IrO}_{2}$.

The $\mathrm{IrO}_{2}$ oxide-based systems are often studied in relation to the oxygen 
evolution reaction. Pfeifer et al. showed the difference between the crystalline rutile $\mathrm{IrO}_{2}$ and amorphous $\mathrm{IrO}_{2}$. In amorphous $\mathrm{IrO}_{2}$ the sharp $t_{2 \mathrm{~g}}$ peak at $529 \mathrm{eV}$ is broadened into a double structure with a new feature at $528 \mathrm{eV}$ [212]. These two features are in two later papers assigned to respectively $\mathrm{O}^{2-}$ and $\mathrm{O}^{1-}$ character $[532,533]$. Within the context on transition metal ions, a more usual assignment would be to assign the new peak at lower energy to a higher valence of the iridium, similar to the assignments in the doped cuprates, etc. When $\mathrm{Ir}$ in $\mathrm{IrO}_{2}$ is partly oxidized to $\mathrm{Ir}^{5+}$ the peaks in the oxygen $\mathrm{K}$ edge will shift to lower energy. The ground state of high valent ions will have strong covalence and part of the hole character will be on oxygen, especially for so-called negative charge transfer ions such as $\mathrm{Cu}^{3+}$ and $\mathrm{Fe}^{4+}$, but to relate this to $\mathrm{O}^{1-}$ is in our view not the best model. In conclusion, we follow a different assignment for the operando oxygen $\mathrm{K}$ edge spectra of $\mathrm{IrO}_{2}$ systems and assign the first peak to the creation of $\mathrm{Ir}^{5+}$ ions.

The iridates are an important group of strongly correlated iridium oxides

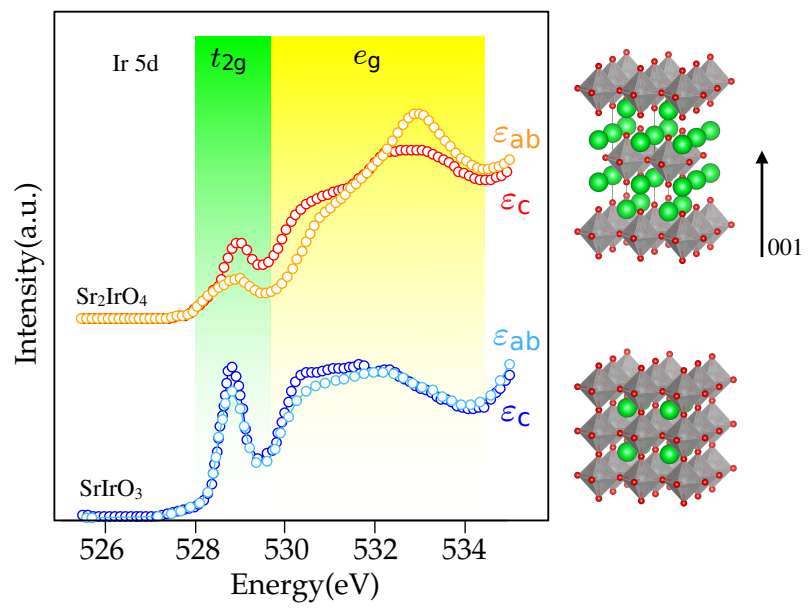

Figure 67: Oxygen $\mathrm{K}$ edge spectra of $\mathrm{Sr}_{2} \mathrm{IrO}_{4}$ layered perovskite compared the $\mathrm{SrIrO}_{3}$ perovskite with the incident electrical field parallel to the layer $\left(\varepsilon_{\mathrm{ab}}\right)$ and perpendicular to the layer $\left(\varepsilon_{\mathrm{c}}\right)[534]$.

with electron correlation properties that are governed by the combination of a large $5 \mathrm{~d}$ spin-orbit coupling and the on-site electron correlation $\mathrm{U}$.

The oxygen $\mathrm{K}$ edge of the $\mathrm{SrIrO}_{3}, \mathrm{Sr}_{2} \mathrm{IrO}_{4}$ and $\mathrm{Sr}_{3} \mathrm{Ir}_{2} \mathrm{O}_{7}$ have been measured on single crystal systems $[534,535]$. Figure 67 compares the angular dependence of the $t_{2 g}$ peak in $\mathrm{SrIrO}_{3}$ versus $\mathrm{Sr}_{2} \mathrm{IrO}_{4}$ and indicates the difference between the $3 \mathrm{D}$ system and the layered system: similarly to the ruthenium layered perovskites, the oxygens are all equivalent in the ${ }_{3} \mathrm{D}$ perovskite, the out-of plane oxygens pointing at the layer can be distinguished from the 
in-plane oxygens.

Platinum oxides: As another noble metal, platinum is largely used as a metal for its surface reactivity. Oxygen $\mathrm{K}$ edge spectroscopy has been used to investigate oxygen interaction with platinum metal surface or interface or the oxides formed.

Platinum occurs in different oxide forms: tetragonal and cubic $\mathrm{PtO}, \mathrm{Pt}_{3} \mathrm{O}_{4}$, $\alpha-\mathrm{PtO}_{2}$ and $\beta-\mathrm{PtO}_{2} \cdot \alpha-\mathrm{PtO}_{2}$ has been measured by Srot et al. [497] in the context of $\mathrm{Pt} / \mathrm{yttrium}$ stabilized zirconia for oxygen electrode reaction. They compare the spectrum with a number of calculations for the other oxides. Guinel et al. studied Pt nanoparticles on carbon nanotubes and they concluded that platinum occurs as $\mathrm{Pt}_{3} \mathrm{O}_{4}$ oxide nanoparticles [536].

\subsection{GENERAL CONSIDERATIONS OF THE 4F OXIDES}

The oxygen K edge spectra of lanthanide (Ln) and actinide (An) oxides are characterized by numerous features corresponding to the oxygen $2 \mathrm{p}$ orbitals hybridized with the $f$ and d orbitals.

Understanding these spectral features aims at determining the $4 / 5^{\mathrm{f}}$ and $5 / 6 \mathrm{~d}$ orbital relative positions, electronic correlations and their role in metaloxygen bonding in relation with the chemical and physical properties. Determination of the nature and changes in the band gap of such materials has also been a motivation.

Most of the lanthanides are non-radioactive and can be handled easily. Hence they have often been considered as models for transuranic elements while experimental studies of actinides are severely limited due to the toxic and radioactive nature of the samples. However, lanthanides and actinides show profound differences in their physico-chemical properties due to the nature of differences between the localization of the $4 \mathrm{f}$ and $5 \mathrm{f}$ orbitals. The differences and similarities in the electronic structures and bonding properties of these f-elements as revealed by oxygen K edge XAS are reviewed.

The systems with an open $4 \mathrm{f}$ shell are known under the names rare earths and lanthanides. Lanthanide atoms have 3 electrons in the $5 \mathrm{~d}, 6 \mathrm{~s}$ and $6 \mathrm{p}$ mixed states and their configuration can be written as $4 \mathrm{f}^{\mathrm{N}}(5 \mathrm{~d} 6 \mathrm{~s} 6 \mathrm{p})^{3}$.

The $4 \mathrm{f}$ orbitals of the lanthanides are localised, while the $5 \mathrm{~d}, 6 \mathrm{~s}$ and $6 \mathrm{p}$ orbitals form bonds with oxygen. This results in their main trivalent oxidation state, where the delocalised electrons fill the oxygen $2 p$ valence band. The localised $4 \mathrm{f}$-states are positioned in between the occupied oxygen $2 \mathrm{p}$ valence and and the empty mixed $5 \mathrm{~d}, 6 \mathrm{~s}$ and $6 \mathrm{p}$ bands. 
Altman et al. studied the oxygen $\mathrm{K}$ edge of the complete series of lan-

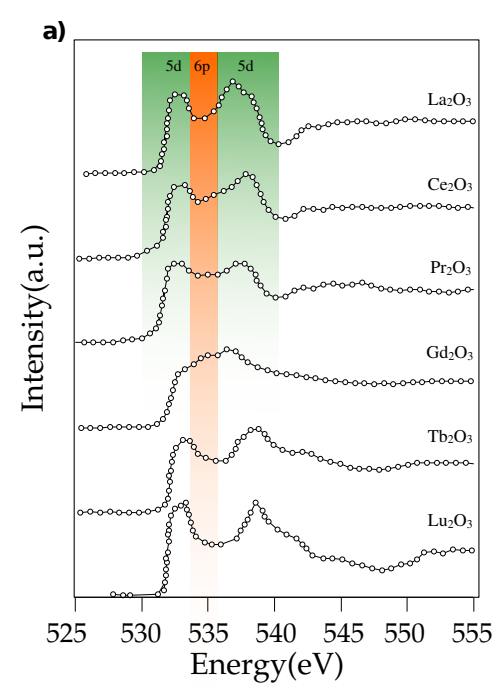

b)

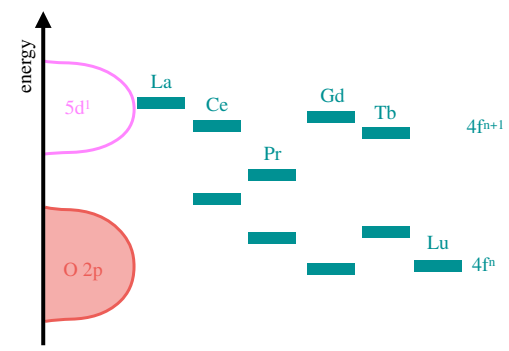

Figure 68: a) Oxygen $\mathrm{K}$ edge spectra of the $\mathrm{Ln}_{2} \mathrm{O}_{3}$ with $\mathrm{Ln}=\mathrm{La}, \mathrm{Ce}, \mathrm{Pr}, \mathrm{Gd}, \mathrm{Tb}$, and $\mathrm{Lu}$ and $\mathrm{b}$ ) the corresponding qualitative energy band diagram adapted from ref. [121].

thanide sesquioxides $\mathrm{Ln}_{2} \mathrm{O}_{3}(\mathrm{Ln}=\mathrm{La}$ to $\mathrm{Lu})$ with STXM and attempted to rationalize the observed spectroscopic variations in relation with the electronic structure of the $\mathrm{Ln}^{3+}$ ions. Two different structures were studied: the hexagonal phases where the $\mathrm{Ln}^{3+}$ ion is 7 -fold coordinated and the cubic phase where the $\mathrm{Ln}^{3+}$ ion is 6-fold coordinated [121].

The oxygen $\mathrm{K}$ edge of six compounds of this series are shown in Figure 68. Starting from the assignment of the transitions observed for $\mathrm{Lu}^{3+}$ in which the $4 \mathrm{f}$ is full, the authors assigned the transitions between $532 \mathrm{eV}$ and $540 \mathrm{eV}$ to oxygen $2 \mathrm{p}$ mixed with lanthanide $5 \mathrm{~d}$ and $6 \mathrm{p}$ states. The $5 \mathrm{~d}$ states are split by the crystal field and the authors distinguished the $5 \mathrm{~d} \pi$ contribution around $532 \mathrm{eV}$ and the $5 \mathrm{~d} \sigma$ contribution around $538 \mathrm{eV}$. The authors analysed the variation of the intensity and position of the $5 \mathrm{~d}$ and $6 \mathrm{p}$ states to deduce the contribution of $4 \mathrm{f}$ states in the assignment of the oxygen $\mathrm{K}$ edge XAS peaks in the other lanthanide compounds using a configuration interaction approach. They discussed the observed abrupt changes in the spectra in terms of orbital hybridization between oxygen $2 \mathrm{p}$ and lanthanide $5 \mathrm{~d}$ in relation to the chemical reactivity of these oxides.

A following study of the $\mathrm{Ce}, \mathrm{Pr}$ and $\mathrm{Tb}$ dioxides (cubic fluorite structure, 8fold coordinated $\mathrm{Ln}^{4+}$ ions) by Minasian et al. [537] revealed drastic changes in the oxygen K edge spectra (Fig. 69) as compared to the trivalent oxides with the contribution of an intense peak around $530 e V$. This feature is as- 

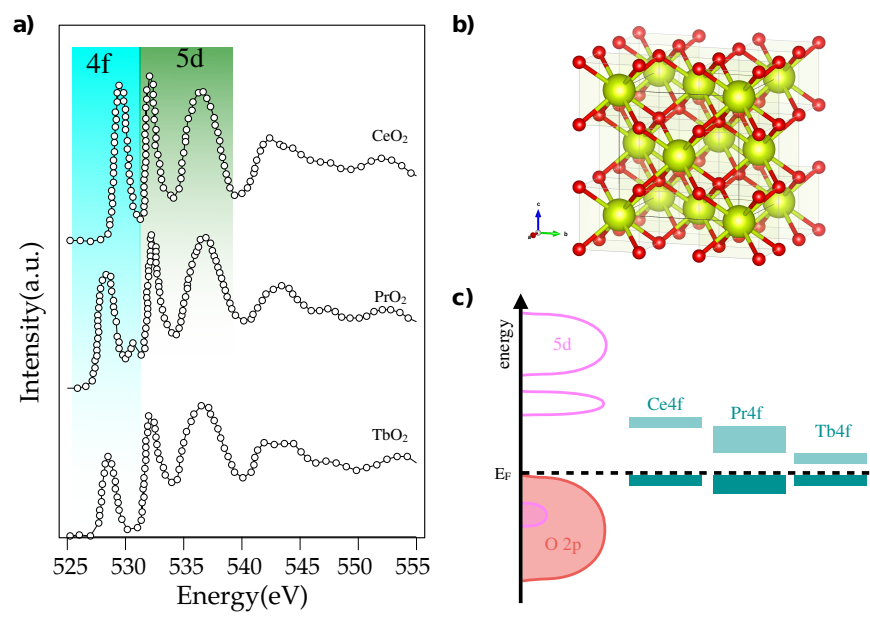

Figure 69: a) Oxygen $\mathrm{K}$ edge spectra of the cubic $\mathrm{CeO}_{2}, \mathrm{PrO}_{2}$ and $\mathrm{TbO}_{2}$ with the corresponding band assignment in $\mathrm{b}$ ) cubic fluorite structure (oxygen atoms in red and lanthanide atoms in yellow) and c) the corresponding schematic DOS adapted from ref. [537].

signed to transitions to the oxygen $2 \mathrm{p}$ states mixed with the $4 \mathrm{f}$ states in light of DOS calculated with VASP. It is followed by the crystal field split $5 \mathrm{~d}$ states. Minasian et al. showed that the orbital mixing between lanthanide $4 \mathrm{f}$ and oxygen $2 \mathrm{p}$ increases in the order $\mathrm{TbO}_{2}$ to $\mathrm{CeO}_{2}$ to $\mathrm{PrO}_{2}$.

Below, the oxygen $\mathrm{K}$ edge studies of lanthanum and cerium oxides are specifically reviewed.

\subsubsection{Lanthanum oxides}

$\mathrm{La}_{2} \mathrm{O}_{3}$ forms cubic and hexagonal crystal structures, where the hexagonal $\mathrm{La}_{2} \mathrm{O}_{3}$ is often studied with respect to its high-k dielectric constant.

Calmels et al. [538] measured the oxygen $\mathrm{K}$ edge of cubic and hexagonal $\mathrm{La}_{2} \mathrm{O}_{3}$. They compared the experiments with both FEFF and Wien $2 \mathrm{~K}$ calculations and reached good agreement. Figure 70 shows the oxygen $\mathrm{K}$ edge spectra of both polymorphs. Cubic $\mathrm{La}_{2} \mathrm{O}_{3}$ (6-fold coordinated La) shows two distinct peaks at 532.4 and $536.8 \mathrm{eV}$, that relate to the oxygen character of the La $\mathrm{e}_{\mathrm{g}}$ and $\mathrm{t}_{2 \mathrm{~g}}$ symmetry $5 \mathrm{~d}$ states, but also containing $6 \mathrm{~s}$ and $6 \mathrm{p}$ character. At higher energies no clear peaks are visible. Hexagonal $\mathrm{La}_{2} \mathrm{O}_{3}$ shows a similar spectrum characterised by two features although broaden and less distinct than in the cubic case.

In both cases, the contribution of the hybridized $4 \mathrm{f}$ states was not ruled out by the authors [121, 538]. 4 f oxides are hygroscopic and tend to form hydroxides in air. The oxygen $\mathrm{K}$ edge of $\mathrm{La}(\mathrm{OH})_{3}$ was measured and calculated by 
Calmels et al. [538]. Lanthanum is present in a series of perovskites, includ-

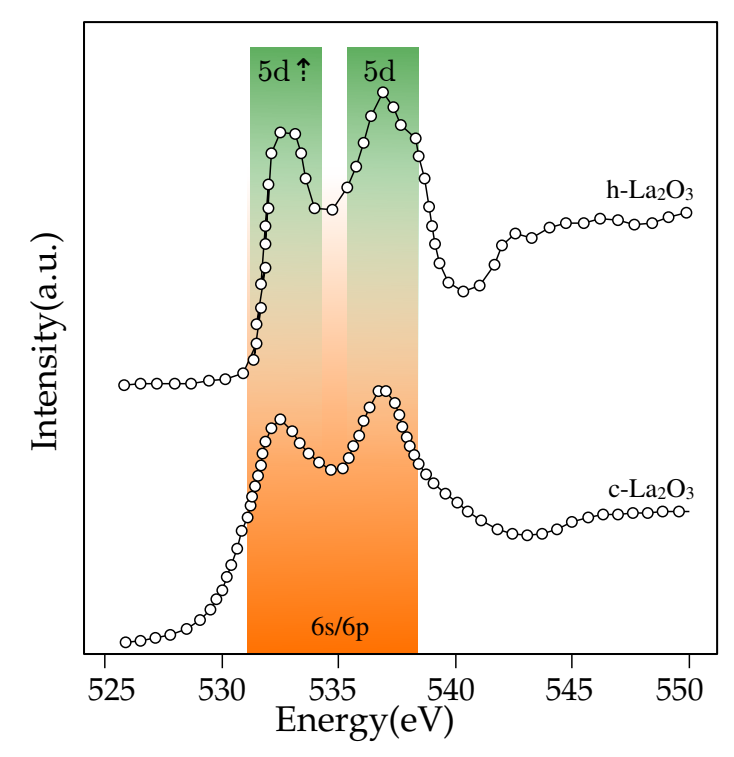

Figure 70: The oxygen $\mathrm{K}$ edge spectrum of cubic $\mathrm{La}_{2} \mathrm{O}_{3}$ [538] and hexagonal $\mathrm{La}_{2} \mathrm{O}_{3}$ [121]

ing $\mathrm{LaMnO}_{3}, \mathrm{LaCoO}_{3}$, etc. and the layered perovskites including $\mathrm{La}_{2} \mathrm{CuO}_{4}$. These $3 \mathrm{~d}$ transition metal oxide systems have been discussed in the $3 \mathrm{~d}$ section as the leading peaks at the edge are the partly empty $3 \mathrm{~d}$ states of the transition metal ion. The lanthanum $5 \mathrm{~d}$-band is always visible at in the 530-540eV energy range. Jeong et al. [539] studied the decomposition of $\mathrm{La}_{2} \mathrm{CuO}_{4}$ into $\mathrm{La}_{2} \mathrm{O}_{3}$ and other oxides with TEM. By comparison to the reference spectra their spectra showed that the decomposition product was hexagonal $\mathrm{La}_{2} \mathrm{O}_{3}$.

\subsubsection{Cerium oxides}

$\mathrm{Ce}_{2} \mathrm{O}_{3}$ is the cerium analog of $\mathrm{La}_{2} \mathrm{O}_{3}$ and it has a $4 \mathrm{f}^{1}$ configuration. The oxygen $\mathrm{K}$ edge of $\mathrm{Ce}_{2} \mathrm{O}_{3}$ is shown in Figure 68 and is similar to that of $\mathrm{La}_{2} \mathrm{O}_{3}$. It is dominated by the $5 \mathrm{~d}, 6 \mathrm{~s}$ and $6 \mathrm{p}$ states [121, 540, 541]. Xu et al. studied mixtures of $\mathrm{Ce}_{2} \mathrm{O}_{3}$ and $\mathrm{CeO}_{2}$ and the occurrence of a small peak before the $\mathrm{Ce}_{2} \mathrm{O}_{3}$ peaks was assigned to $\mathrm{CeO}_{2}$ impurities. [540].

In $\mathrm{CeO}_{2}, \mathrm{Ce}^{4+}$ has $4 \mathrm{f}^{0} \mathrm{~d}^{0}$ electronic configuration. The oxygen $\mathrm{K}$ edge of cubic $\mathrm{CeO}_{2}$ has been often measured both with XAS and EELS [540-546]. The spectrum is shown in Figure 69. The presence of a strong 4 f-related 
peak highlights the non-negligible hybridization between oxygen $2 p$ and $\mathrm{Ce} 4 \mathrm{f}$ orbitals. The crystal field in cerium dioxide splits the $5 \mathrm{~d}$ states but the splitting of the $4 \mathrm{f}$ states is not observed in agreement with the small overlap between the $\mathrm{f}$ orbitals and the ligands in lanthanides. The crystal field splitting of the $5 \mathrm{~d}$ states is $4.3 \mathrm{eV}$ for $\mathrm{CeO}_{2}$.

Mullins et al. reported spectra for cerium oxides thin films used as an important component in automotive exhaust catalysts [547]. Wang et al. studied the mixture of cerium and terbium oxides [545]. Garvie and Buseck showed that $\mathrm{CeO}_{2}$ changes to $\mathrm{Ce}_{2} \mathrm{O}_{3}$ in the electron beam [541].

\subsection{GENERAL CONSIDERATIONS OF THE 5F OXIDES}

With their relatively delocalised $5 \mathrm{f}$ valence electrons, actinides show a rich molecular chemistry, with oxidation states spanning from $3+$ to $7+$. In particular, actinides in high oxidation states $5^{+}$and $6+$, can occur as a linear transdioxo actinyl cation, with two short highly covalent $\mathrm{An}=\mathrm{O}$ bonds. These bonds are often considered as chemically inert. The actinyl cation forms bipyramidal polyhedra by bonding various number of equatorial ligands. Oxygen $\mathrm{K}$ edge XAS is used to provide insights into the fundamental understanding of the participation of $5 \mathrm{f}$ and $6 \mathrm{~d}$ orbitals to chemical bonding. Figure 71 presents the spectra of the light actinide dioxides: $\mathrm{UO}_{2}, \mathrm{NpO}_{2}$, and $\mathrm{PuO}_{2}$ all in the cubic fluorite structure.

In light of $\mathrm{DFT}+\mathrm{U}$ calculations, Modin et al. used oxygen $\mathrm{K}$ edge XAS to compare the nature of the conduction band of these compounds [548]. The oxygen K edge XAS spectra of the three actinides show a first peak assigned to oxygen $2 \mathrm{p}$ states hybridized with An $5^{f}$ and two peaks assigned to hybridized An 6d-O 2p bands split by crystal field. Differences in the XAS spectra arise from the relative position of the empty $5 \mathrm{f}$ and $6 \mathrm{~d}$ bands. While in $\mathrm{UO}_{2}$, the ${ }^{\mathrm{f}}$ and first $6 \mathrm{~d}\left(\mathrm{e}_{\mathrm{g}}\right)$ band overlap, it appears that when changing from $\mathrm{U}$ to $\mathrm{Np}$ to $\mathrm{Pu}$, the $5 \mathrm{f}$ band is lowered in energy and separates from the first $6 \mathrm{~d}\left(\mathrm{e}_{\mathrm{g}}\right)$ band, in agreement with the increase of the number of $5 \mathrm{f}$ electrons from 2 in $\mathrm{U}^{4+}$ to 4 in $\mathrm{Pu}^{4+}$.

Oxygen $\mathrm{K}$ edge spectroscopy was used to probe the oxidation state of $\mathrm{Pu}$ in the $\mathrm{PuO}_{2}$ single crystal [549]. Oxygen $\mathrm{K}$ edge XANES measured with STXM was applied to the characterization of sonochemical Pu colloids [550]. The spatial resolution offered by the STXM technique, enabled to reveal the contribution of the hydrolyzed species in hydrolytic colloids compared with sonochemical colloids. 


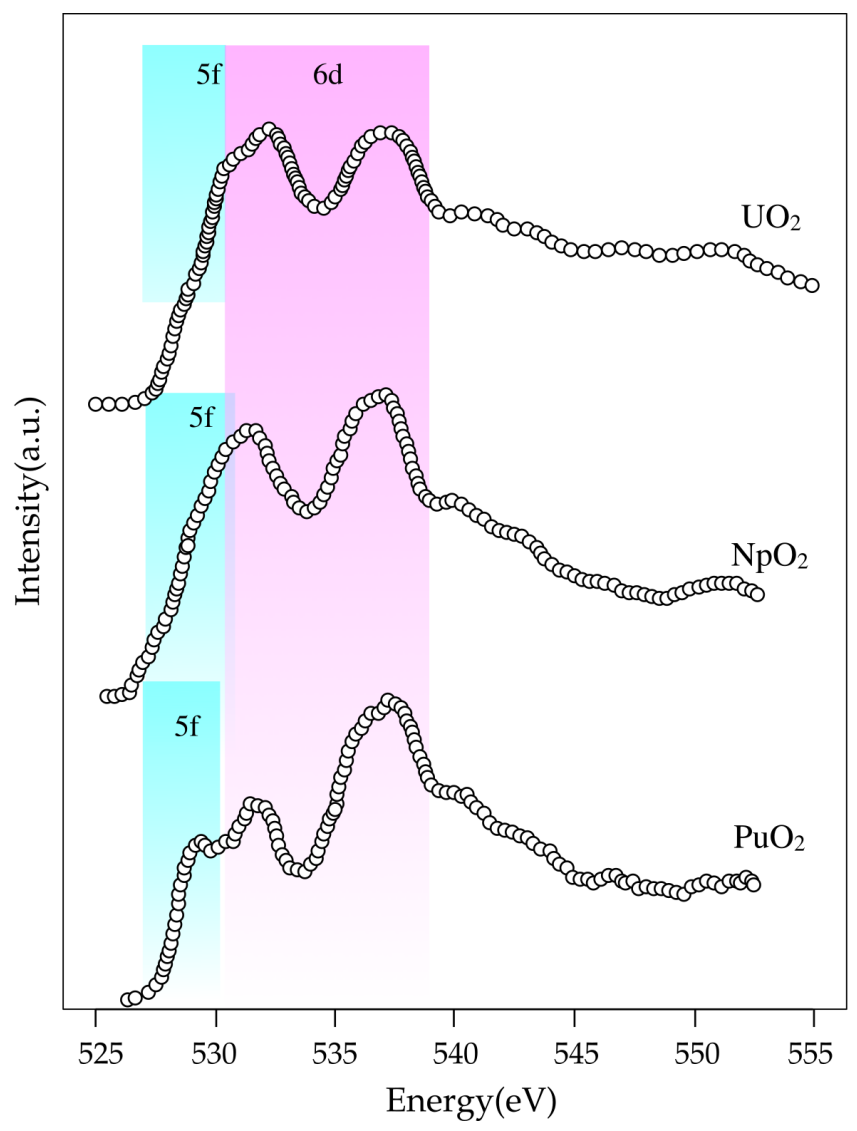

Figure 71: Oxygen $\mathrm{K}$ edge spectra of $\mathrm{UO}_{2}$ [551], $\mathrm{NpO}_{2}$ [552] and $\mathrm{PuO}_{2}$ [552].

\subsubsection{Uranium oxides}

Thanks to its early discovery and high relative abundance, in addition to the relatively low activity of its radioisotopes, uranium is the most studied and best known actinide element. Understanding fully its chemical reactivity is of relevance for industrial, environmental and health purposes. Efforts were dedicated to determine the contribution of the $5 \mathrm{f}$ and $6 \mathrm{p}$ valence electrons to the chemical bonds. We discuss first the binary oxide $\mathrm{UO}_{2}$ and subsequently the $\mathrm{U}^{6+}$ uranyl molecular complexes.

$\mathrm{UO}_{2}$ is an important compound for nuclear fuel processing as well as a reference compound for the study of the fundamental physics of $f$ electrons. The determination of the nature of the bottom of the conduction band is another important step in the physics of $\mathrm{UO}_{2}$. Although known to be a Mott-Hubbard insulator, several studies aimed at determining between the $\mathrm{f}-\mathrm{f}$ or $\mathrm{f}-\mathrm{d}$ type. This information is relevant to understand the poor thermal conductivity of $\mathrm{UO}_{2}$ as well as testing the first-principle theoretical band structure predictions. Both information being critical for the management of nuclear power plants. However, an experimental proof is required to val- 
idate the first-principle calculations.

$\mathrm{UO}_{2}$ has formally the $5 \mathrm{f}^{2} 6 \mathrm{~d}^{0}$ electronic configuration where $\mathrm{U}^{4+}$ is in a cubic site with 8 equivalent $\mathrm{U}-\mathrm{O}$ bonds. The crystal field in uranium splits the $6 \mathrm{~d}$ states by $4.8 \mathrm{eV}$ [543] . The oxygen $\mathrm{K}$ edge of $\mathrm{UO}_{2}$ (Fig.7I) analysed by multiple-scattering and LSDA+U calculations [543, 544] provided proof of the f-f type Mott-Hubbard insulator.

The oxygen K edge XAS spectra have been calculated with LDA+U calculations [548]. The oxygen $\mathrm{K}$ edge XAS has been used to study the evolution of $\mathrm{UO}_{2}$-based nuclear fuel under irradiation. An early study of $\mathrm{UO}_{2}$ pellets implanted with Mo ions used the low penetration depth of oxygen K edge XAS [551] to probe the oxidation state of uranium at the implanted surface. The observation of a low energy resonance after annealing was assigned to the presence of interstitial oxygens and the hyperstoichiometric nature of the surface. The thesis of Pizzi [553] on the influence of the partial pressure of oxygen on the oxidation state of uranium in $\mathrm{UO}_{2}$, used the oxygen $\mathrm{K}$ edge to investigate the contribution of oxidized phases. Pizzi highlighted the difference between oxygen K edge data obtained in TEY vs TFY modes: the TEY data from Wu et al. contain the contribution of an oxidized layer, which is absent in the data measured in TFY.

In ambient conditions, uranium is most stable in its oxidized $\mathrm{U}^{6+}$ uranyl

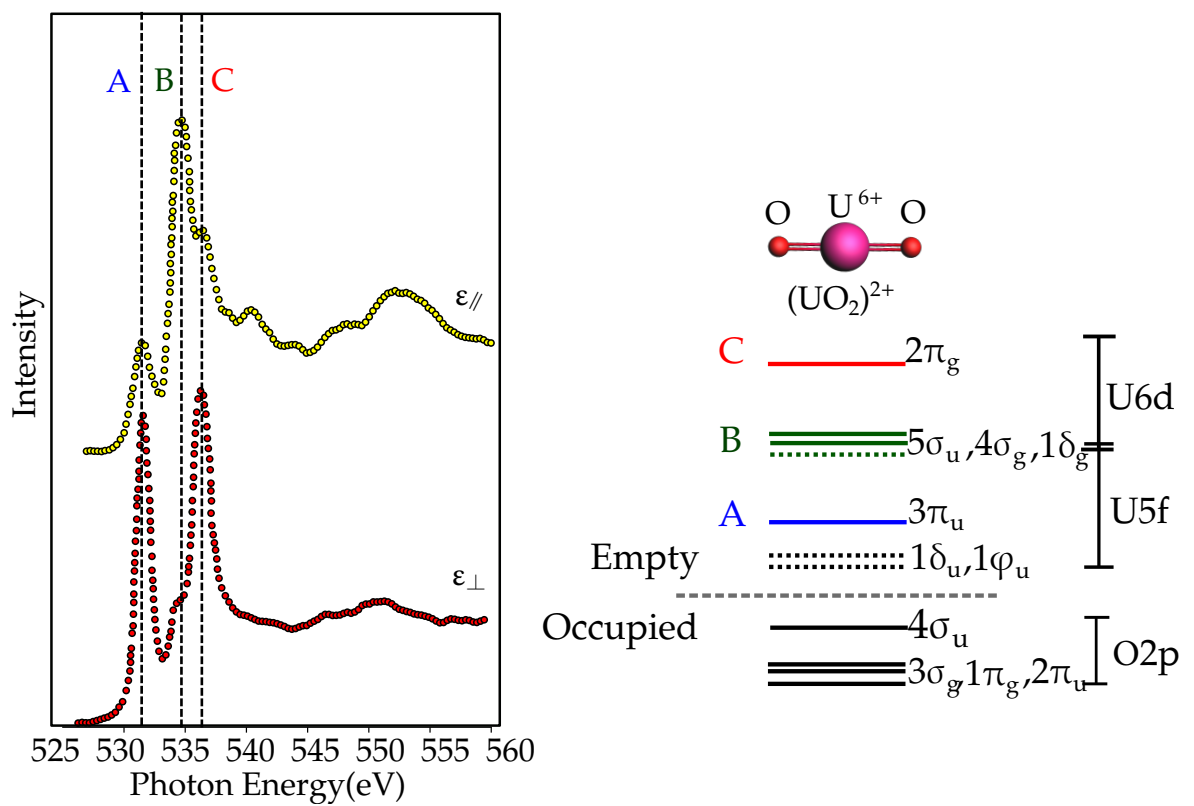

Figure 72: The polarized oxygen $\mathrm{K}$ edge spectrum of the uranyl $\mathrm{UO}_{2}^{2+}$ species in $\mathrm{Cs}_{2} \mathrm{UO}_{2} \mathrm{Cl}_{4}$ single crystal [552]. The $\sigma$ and $\pi$ character of the probed states are revealed with the incident light polarisation respectively parallel to the uranyl bond and perpendicular. 
state and tends to form strongly covalent linear trans-dioxo uranyl species. Understanding the electronic structure of the uranyl species has been the subject of many investigations to determine its reactivity.

Denning et al. used linear polarized x-rays to distinguish between $\sigma$ (parallel to the uranyl bond) and $\pi$ (perpendicular to the uranyl bond) orbital symmetry of the antibonding orbitals of the uranyl in a $\mathrm{Cs}_{2} \mathrm{UO}_{2} \mathrm{Cl}_{4}$ single crystals, which contains oxygen ligands only in the uranyl species (Fig. 72). Comparison of the parallel and perpendicular polarized spectra with the molecular orbital diagram of the uranyl cation calculated by DFT with the ADF code, allows assigning the three main peaks of the oxygen $\mathrm{K}$ edge XAS spectrum to $3 \pi_{\mathfrak{u}}\left(5 \mathrm{f}_{\pi}\right), 5 \sigma_{\mathfrak{u}}\left(5 \mathrm{f}_{\sigma}\right), 4 \sigma_{g}\left(6 \mathrm{~d}_{\sigma}\right)$, and $2 \pi_{\mathrm{g}}\left(6 \mathrm{~d}_{\pi}\right)$ [552]. A following study of these antibonding valence states of $\mathrm{U}^{6+}$ was performed on $\mathrm{Ba}_{2} \mathrm{ZnUO}_{6}$ where all oxygen in the $\mathrm{U}$ coordination sphere are equivalents. Experimental spectrum was compared to FDMNES calculations using the finite-difference method [554]. Fillaux et al. studied specifically the effect of the equatorial ligands on the trans-dioxo bonds using non-oxygen equatorial ligands [555]. Oxygen $\mathrm{K}$ edge spectra were calculated with FDMNES and the finite-difference method where the electronic configuration was obtained from Mulliken population analysis performed with ADF. Polarized calculations enable distinguishing the antibonding $\pi^{*}$ from the $\sigma^{*}$ orbitals and pDOS calculations allow assigning the transitions to the $5 \mathrm{f}$ and $6 \mathrm{~d}$. The intensity of oxygen $\mathrm{K}$ edge XANES features can be used as a fingerprint for the hybridization strength. Fillaux et al. showed that the nature of the equatorial ligands modifies strongly the relative energies of the antibonding molecular orbitals.

In the field of geochemistry, Ward et al. published a compilation of oxygen $\mathrm{K}$ edge data of common uranyl-bearing minerals, including carbonates, oxyhydroxides, phosphates and silicates. All spectra present a first sharp peak assigned to the $3 \pi_{u}\left(5 f_{\pi}\right)$ of the uranyl and other features at higher energy depending on the compound. The authors showed that the uranyl compounds can be distinguished based on their oxygen $\mathrm{K}$ edge spectra. This opens perspectives for mineral identification using STXM [120]. 

The omnipresence of oxygen in our universe ensures that this light element never stops to draw the attention of the scientific community.

The experimental instrumental advancements in the 8o's enabled the first determination of oxygen $\mathrm{K}$ edge spectra in a range of energies inaccessible before.

Little is changed from an experimental point of view in terms of data quality but the plethora of experimental and theoretical techniques made sure that the systems exploration from an oxygen point of view keeps on attracting the attention of many. In this thesis we analyze and describe x-ray oxygen $\mathrm{K}$ edge investigations in molecular and solid systems without leaving aside experimental and theoretical key aspects.

Furthermore we investigate in details two popular approaches to the modelling of K edges in molecular and solid systems: the coupled cluster hierarchy of methods and the DFT-based $\triangle \mathrm{SCF}$ method.

\section{Part I}

The computational procedures used for the modelling of core level processes underwent great improvements during the last decades. There is a multitude of available approaches tailored for different systems and, despite the controversy regarding the individual performance of different methods, the common fundamental issue is the computational efficiency.

This ensures that the choice of the procedure to obtain core level spectra should always take into account the size of the system under investigation. The methodologies discussed in this thesis range from those with wider applicability like DFT-based methods in the framework of the single particle approximation to many body approaches as BSE and TDDFT.

The discussion includes also multiplet calculations, that have shown good performances with a reduced computational time, and post-Hartree Fock multiconfigurational methods.

Despite the availability of many approaches to the computation of core excited spectra, one of the main issue is the treatment of the core hole and 
the best way to take into account core hole effects. If for some systems good results can be obtained by reducing the occupation of the absorbing atom in a supercell within the standard DFT framework, other systems require rigorous approaches as BSE and TDDFT that include many body effects. From an experimental point of view, the fundamental ingredients to measure oxygen $\mathrm{K}$ edges are an $\mathrm{x}$-ray source, a monochromator and a detector and the available techniques range from the most commonly used EELS to femtosecond XFEL state of art techniques.

The is lifetime constitute an intrinsic limitation in the resolution of the spectral features but the emerging of cutting edge technologies in the field of HHG sources, XFEL sources, RIXS beamlines and x-ray microscopes will eventually lead to a deeper understanding of the dynamics of the electronic structures of oxygen containing systems.

Part II In Chapter 2 we report the results for atomic and ionized oxygen: atomic oxygen photoionization and photoabsorption are fundamental astrophysical processes and a huge amount of experimental and theoretical works have been carried out in order to elucidate these processes.

However, despite their popularity, it is not straightforward to find models that accurately describe inner-shell photoexcitation because of a variety of relaxation phenomena that make their description tricky.

The discussion then moves to molecular systems containing oxygen, starting from small binary molecules to large bio molecular objects. The investigation of the oxygen $\mathrm{K}$ edge on small molecules containing oxygen helped establishing reference models for the study of organic macromolecules where the analysis is complicated by beam damage.

The various degradation phenomena are discussed in relation to the literature. If the x-ray results for small molecules such as $\mathrm{CO}, \mathrm{NO}, \mathrm{CO}_{2}$ and $\mathrm{NO}_{2}$ are well understood and not many questions are left without an answer, this is not the case for water, where the large amount of literature on the topic well documents many debates and open questions regarding both the liquid and the solid states of water.

The discussion from an atomic and molecular point of view of the oxygen $\mathrm{K}$ edge clear the way for the analysis of the molecular and atomic adsorption phenomena where the oxygen $\mathrm{K}$ edge investigation supplies an useful tool to detect the changes induced by the adsorption process itself. The versatility of the x-ray analysis combined with the omnipresence of oxygen made sure that the study of catalytic reactions advanced also thanks to the in situ applicability. 
In Chapter 3 we present an investigation on the performance of a corevalence separated scheme within the coupled cluster $(C C)$ hierarchy of methods CC singles (CCS), CC singles and approximate doubles (CC2) and CC singles and doubles (CCSD) in reproducing the $\mathrm{K}$ edge $\mathrm{x}$-ray absorption spectra of the low- $\mathrm{Z}$ elements carbon, oxygen and fluorine in formaldehyde $\left(\mathrm{CH}_{2} \mathrm{O}\right)$, carbonyl fluoride $\left(\mathrm{CF}_{2} \mathrm{O}\right)$, formyl fluoride $(\mathrm{CHFO})$ and formic acid $(\mathrm{CHOOH})$. The modelling within the same molecule of all the $\mathrm{K}$ edges gives a comprehensive characterization of the electronic structure and highlights the optimal theoretical framework for the core excited states description. The analysis covers the entire frequency region from the first $1 \mathrm{~s} \rightarrow \pi^{*}$ excitation to the core-ionization limit, encompassing the region of Rydberg transitions.

The adoption of different basis sets with and without core polarization functions, diffuse functions and Rydberg treatment combined with different level of theory provides a deep analysis aimed at track down the fundamental ingredients and their balancing for an optimal description of the excited states of the system. Moreover, a simulation of the vibronic progressions in the $1 s \rightarrow \pi^{*}$ bands of both carbon and oxygen in formaldehyde has been performed with the $\mathrm{AH}$ harmonic model, on the grounds of (equation of motion)EOM-CCSD quadratic expansions of the ground and excited states. The vibronic analysis not only completes the spectra description but it is also a test for the chosen methodology since the quality of the results depends on the gradient and the hessian. Even if a good agreement with the experiment is reached we highlight the necessity to include the triple excitations treatment in the coupled cluster operator and we trace back the subtle discrepancies with the experiment to the underestimation of the $\mathrm{CO}$ bond elongation in the excited state.

Part III The last part of this thesis deals with solid oxides results.

Chapter 4 starts with an excursus on oxygen $\mathrm{K}$ edge results on alkaline metal oxides and then Chapter 5 goes into details regarding the modelling of oxygen $\mathrm{K}$ edge spectra in alkaline earth metal oxides.

First, a ground state analysis of the alkaline earth metal oxides highlights their electronic structure through an examination of the partial density of states in order to highlight the differences in the atomic composition of bands above and below the Fermi energy. Furthermore the Wannier functions obtained for the oxygen $2 p$ states provides a pictorial representation of the orbitals localization rationalized by the hopping frequency integrals. A comparison between the available experimental data and the calculated 
ones by a $\Delta$ self consistent field $(\triangle \mathrm{SCF})$ excited core hole $(\mathrm{XCH})$ is reported. The discrepancies between some of the experimental data regarding $\mathrm{CaO}$, $\mathrm{SrO}$ and $\mathrm{BaO}$ and the theoretical data are traced back to possible carbonates and hydroxides contamination due to the highly reactive surfaces of those materials.

The oxygen K-edge spectra obtained for the corresponding carbonates and hydroxides reinforce the hypothesis that some of the experimental data are affected by contamination. The comparison of the spectra obtained with and without the core hole highlights the necessity of an inclusion of the core hole treatment for the lighter oxides in the series $(\mathrm{MgO}$ and $\mathrm{CaO})$ where for $\mathrm{SrO}$ and $\mathrm{BaO}$ the omission of the core hole treatment already gives a qualitative description of the system in terms of spectral features and peak positions. The electron localization plays a role in the extent of the core hole effects in terms of screening efficiency. A localized character of electron distribution is an evidence of a small bonding overlap leading to a reduced degree of covalency of the metal-oxygen bond. In order to further investigate the bonding character in the alkaline earth metal oxide series the Bader analysis has been performed and the result indicates a high degree of ionicity for all the systems with $\mathrm{MgO}$ as the most ionic.

The chemical shift obtained as difference between the energy of the system with an oxygen is core hole and the energy of the ground state reveals a stronger bound oxygen is electron going from $\mathrm{MgO}$ to $\mathrm{BaO}$. In conclusion, not only we highlights the necessity for the revision of some of the experimental data but we also further investigate the nature of the alkaline earth metal-oxygen bond concluding that it is indeed true that all the alkaline earth metal oxides are strongly ionic materials but the covalent component extent in the O-M bond goes up down the series.

In Chapter 6 the discussion moves on the left side of the periodic table toward the big family of $f$ and $d$ metal oxides. First the general analysis of the oxygen $\mathrm{K}$ edges of $3 \mathrm{~d}$ transition metal oxides is introduced.

We start the discussion with the crystal field and the exchange splitting and the influence of the covalency on the trend of the $3 \mathrm{~d}$ series spectral shape. Next the discussion moves to binary oxides with rocksalt (MO), the corundum $\mathrm{M}_{2} \mathrm{O}_{3}$ and rutile $\mathrm{MO}_{2}$ and their trends in spectral shape as function of oxidation state.

The line of reasoning finally leads to a element by element treatise. The $4 \mathrm{~d}$ oxides stand in need for a different disquisition with respect to their $3 \mathrm{~d}$ counterpart because of a larger crystal splitting $(3.5 \mathrm{eV})$ that implies a low-spin state. The oxygen $\mathrm{K}$ edge spectra of lanthanide and actinide are 
reported and their study aims at the determination of the $4 / 5 \mathrm{f}$ and $5 / 6 \mathrm{~d}$ orbital relative positions, electronic correlations and their role in metal-oxygen bonding in relation with the chemical and physical properties.

In summary, x-ray experiments at the oxygen $\mathrm{K}$ edge will in the future benefit from instrumental developments in spatial and time resolution. The great potential of $x$-ray spectroscopies combined with the omnipresence of oxygen in our universe will offer a great potential to gain understanding of the electronic structure of many oxygen containing systems. Simultaneously theoretical predictions and simulations will guide the interpretation of new data leading to new exciting outcomes from the x-ray investigation from an oxygen point of view. 
De alomtegenwoordigheid van zuurstof in ons universum maakt dat dit lichte element altijd de aandacht van de wetenschappelijke gemeenschap weet te trekken. De vooruitgang in experimentele instrumentatie in de jaren 80 maakte het mogelijk om voor het eerst spectra op te nemen van de zuurstof K-edge bij energieën die tot die tijd ontoegankelijk waren.

Sinds deze experimenten is er weinig veranderd aan de kwaliteit van de data maar de overvloed aan experimentele en theoretische technieken heeft ervoor gezorgd dat de systeemverkenning vanuit het perspectief van zuurstof de aandacht van velen heeft blijven trekken. In dit proefschrift analyseren en beschrijven we röntgenonderzoeken van de zuurstof K-edge in moleculaire en vaste systemen zonder daarbij belangrijke experimentele en theoretische aspecten buiten beschouwing te laten. Verder onderzoeken we in detail twee populaire benaderingen voor het modelleren van K-edges in moleculaire en vaste systemen: de hiërarchie van coupled cluster methoden en de op DFT gebaseerde $\triangle$ SCF-methode.

Deel I De computationele procedures die worden gebruikt voor het modelleren van processen op kernniveau zijn de afgelopen decennia sterk verbeterd. Er is een groot aantal benaderingen beschikbaar die zijn toegesneden op verschillende systemen. Er zijn veel discussies over de individuele prestaties van deze methoden maar het gemeenschappelijke fundamentele probleem is de rekenefficiëntie. Om deze reden moet bij de keuze van de procedure altijd rekening worden gehouden met de grootte van het onderzochte systeem. De methodologieën die in dit proefschrift worden besproken variëren van methoden die breder toepasbaar zijn, zoals op DFT gebaseerde methoden die een enkelvoudigedeeltjesbenadering gebruiken, tot meerlichamenbenaderingen zoals BSE en TDDFT. De discussie omvat ook multipletberekeningen, die goede prestaties leveren met een kortere rekentijd, en post-Hartree-Fock-multiconfiguratiemethoden. Ondanks de vele mogelijke benaderingen voor de berekening van kerngeëxciteerde spectra zijn er nog altijd computationele problemen. De belangrijkste hiervan zijn de behandeling van het kerngat en de beste manier om rekening te houden met kerngateffecten. Voor sommige systemen kunnen goede resultaten worden verkregen door de bezetting van het absorberende atoom in een supercel te verminderen binnen het standaard DFT-raamwerk. Andere systemen vereisen rigoureuze benaderingen waar meerlichameneffecten zijn 
meegenomen, zoals BSE en TDDFT. Vanuit experimenteel oogpunt zijn de fundamentele benodigdheden voor het meten van zuurstof K-edges een röntgenbron, een monochromator en een detector. De beschikbare technieken variëren van de meest gebruikte EELS tot de nieuwste femtoseconde XFELtechnieken. De resolutie van de spectrale kenmerken wordt intrinsiek beperkt door de is levensduur, maar de opkomst van geavanceerde technologieën op het gebied van HHG-bronnen, XFEL-bronnen, RIXS-bundellijnen en röntgenmicroscopen zal uiteindelijk leiden tot een dieper inzicht in de dynamiek van de elektronische structuren van zuurstofhoudende systemen.

\section{Deel II}

In Hoofdstuk 2 rapporteren we de resultaten voor atomaire en geïoniseerde zuurstof: atomaire zuurstof foto-ionisatie en fotoabsorptie zijn fundamentele astrofysische processen en er is een enorme hoeveelheid experimenteel en theoretisch onderzoek uitgevoerd om deze processen te begrijpen. Ondanks deze populariteit is het niet eenvoudig om modellen te vinden die de fotoexcitatie van de binnenste schil nauwkeurig beschrijven vanwege een verscheidenheid aan relaxatieverschijnselen die hun beschrijving lastig maken. De discussie gaat vervolgens over naar moleculaire systemen die zuurstof bevatten, van kleine binaire moleculen tot grote biomoleculaire objecten. Het onderzoek van de zuurstof K-edges op kleine moleculen die zuurstof bevatten hielp bij het opstellen van referentiemodellen voor de studie van organische macromoleculen, waar de analyse gecompliceerd wordt door degradatie geïnduceerd door de röntgenbundel. De verschillende degradatieverschijnselen worden besproken in relatie tot de literatuur. De röntgenresultaten voor kleine moleculen zoals $\mathrm{CO}, \mathrm{NO}, \mathrm{CO}_{2}$ en $\mathrm{NO}_{2}$ worden goed begrepen en er blijven niet veel vragen onbeantwoord. Dit is niet het geval voor water, waar de grote hoeveelheid literatuur over het onderwerp en goed overzicht geeft van vele debatten en open vragen over zowel de vloeibare als de vaste toestand van water. In een vervolgonderzoek wordt gekeken naar de analyse van moleculaire en atomaire adsorptieverschijnselen. Zuurstof K-edge spectra zijn een nuttig hulpmiddel om veranderingen te detecteren die worden veroorzaakt door het absorptieproces. De veelzijdigheid van de röntgenanalyse in combinatie met de toepasbaarheid van de techniek in situ en de alomtegenwoordigheid van zuurstof zorgde ervoor dat de studie van katalytische reacties vorderde. 
In Hoofdstuk 3 presenteren we een onderzoek naar de prestaties van een kern-valentie-gescheiden schema binnen de coupled cluster (CC) hiërarchie van methoden "CC singles" (CCS), "CC singles and approximate doubles" (CC2) en "CC singles and doubles" (CCSD) in het reproduceren van de K-edge röntgenabsorptiespectra van de lage-Z-elementen koolstof, zuurstof en fluor in formaldehyde $\left(\mathrm{CH}_{2} \mathrm{O}\right)$, carbonylfluoride $\left.\mathrm{CF}_{2} \mathrm{O}\right)$, formylfluoride $(\mathrm{CHFO})$ en mierenzuur $(\mathrm{CHOOH})$. De modellering binnen hetzelfde molecuul van alle K-edges resulteert in een uitgebreide karakterisering van de elektronische structuur en benadrukt het optimale theoretische kader voor de beschrijving van de kernaangeslagen toestanden. De analyse dekt het hele frequentiegebied vanaf de eerste $1 \mathrm{~s} \longrightarrow(\mathrm{pi})^{*}$ excitatie tot de kernionisatielimiet, die het gebied van Rydberg-overgangen omvat. Het gebruik van verschillende basissets met en zonder kernpolarisatiefuncties, diffuse functies en Rydberg-behandeling in combinatie met verschillende theorieniveaus biedt een diepgaande analyse gericht op het opsporen van de fundamentele componenten en hoe deze gebalanceerd worden voor een optimale beschrijving van de geëxciteerde toestanden van het systeem. Bovendien is een simulatie van de vibronische progressies in de $1 \mathrm{~s} \longrightarrow(\mathrm{pi})^{*}$ banden van zowel koolstof als zuurstof in formaldehyde uitgevoerd met het $\mathrm{AH}$-harmonische model, op grond van (bewegingsvergelijking)EOM-CCSD kwadratische expansie van de grond- en aangeslagen toestanden. De vibronische analyse maakt de beschrijving van de spectra niet alleen compleet maar is ook een test voor de gekozen methodologie, aangezien de kwaliteit van de resultaten afhangt van de gradiënt en de Hessiaan. Zelfs als er een goede overeenstemming met het experiment wordt bereikt, benadrukken we de noodzaak om de drievoudige excitaties op te nemen in de coupled cluster-operator. We herleiden de subtiele afwijkingen met het experiment tot de onderschatting van de toename in CO-bindingslengte in de aangeslagen toestand.

\section{Deel III}

Het laatste deel van dit proefschrift beschrijft de resultaten van vaste oxiden. Hoofdstuk 4 begint met een uitleg over zuurstof K-edge-resultaten van alkalimetaaloxiden en vervolgens gaat hoofdstuk 5 in op details over de modellering van zuurstof K-edge-spectra van aardalkalimetaaloxiden. Een analyse van de grondtoestand van de aardalkalimetaaloxiden benadrukt hun elektronische structuur. Deze analyse is gedaan door de partiële dichtheid van toestanden te onderzoeken om de verschillen in de atomaire samenstelling van banden boven en onder de Fermi-energie te benadrukken. Verder 
bieden de Wannier-functies die zijn verkregen voor de zuurstof $2 p$-toestanden een illustratie van de lokalisatie van de orbitalen, gerationaliseerd door de hopping-frequentie-integralen. Een vergelijking wordt gerapporteerd tussen de beschikbare experimentele gegevens en berekeningen met een zelfconsistent veld (SCF) geëxciteerd kerngat $(\mathrm{XCH})$. De afwijkingen tussen enkele van de experimentele gegevens met betrekking tot $\mathrm{CaO}, \mathrm{SrO}$ en $\mathrm{BaO}$ en de theoretische gegevens zijn terug te voeren op mogelijke carbonaten en hydroxidenverontreinigingen als gevolg van de zeer reactieve oppervlakken van die materialen. De zuurstof K-edge-spectra verkregen voor de bijbehorende carbonaten en hydroxiden versterken de hypothese dat sommige van de experimentele gegevens worden beïnvloed door verontreinigingen. De vergelijking van de spectra verkregen met en zonder het kerngat benadrukt de noodzaak van het meenemen van de behandeling van het kerngat voor de lichtere oxiden in de reeks $(\mathrm{MgO}$ en $\mathrm{CaO})$, terwijl voor $\mathrm{SrO}$ en $\mathrm{BaO}$ het weglaten van deze behandeling al een kwalitatieve beschrijving van het systeem geeft in termen van spectrale kenmerken en piekposities. De elektronenlokalisatie speelt een rol in de omvang van de kerngateffecten in termen van screeningefficiëntie. Een gelokaliseerd karakter van elektronendistributie is bewijs voor een kleine bindingsoverlap wat leidt tot een verminderde mate van covalentie van de metaal-zuurstofbinding. Om het bindingskarakter in de aardalkalimetaaloxidereeks verder te onderzoeken is de Bader-analyse uitgevoerd. Het resultaat wijst op een hoge mate van ioniciteit voor alle systemen met $\mathrm{MgO}$ als het meest ionische. De chemische verschuiving verkregen als verschil tussen de energie van het systeem met een zuurstof is kerngat en de energie van de grondtoestand onthult dat het zuurstof is elektron sterker gebonden is wanneer men van $\mathrm{MgO}$ naar $\mathrm{BaO}$ gaat. Concluderend benadrukken we niet alleen de noodzaak van de herziening van sommige van de in de literatuur gerapporteerde experimentele gegevens maar we onderzoeken ook de aard van de aardalkalimetaal-zuurstofbinding verder. We concluderen dat het inderdaad waar is dat alle aardalkalimetaaloxiden sterk ionische materialen zijn maar dat de omvang van de covalente component in de O-M-binding toeneemt naarmate men afdaalt in de groep.

In hoofdstuk 6 verplaatst de discussie zich aan de linkerkant van het periodiek systeem naar de grote familie van $\mathrm{f}$ - en $\mathrm{d}$-metaaloxiden. Eerst wordt de algemene analyse van de zuurstof K-edges van $3 \mathrm{~d}$-overgangsmetaaloxiden geïntroduceerd. We beginnen de discussie met het kristalveld en de exchange splitting en de invloed van de covalentie op de trend van de spec- 
trale vorm van de 3 d-reeks. Vervolgens gaat de discussie over binaire oxiden met steenzout (MO), korund $\mathrm{M}_{2} \mathrm{O}_{3}$ en rutiel $\mathrm{MO}_{2}$ en hun trends in spectrale vorm als functie van de oxidatietoestand. De redenering leidt uiteindelijk tot een behandeling element voor element. De 4 d-oxiden hebben een andere weergave nodig dan hun 3 d-tegenhangers vanwege een grotere kristalsplitsing $(3,5 \mathrm{eV})$ die een lage-spintoestand impliceert. De zuurstof K-edgespectra van lanthanide en actinide worden gerapporteerd. Deze studie is gericht op de bepaling van de relatieve posities van $4 / 5 \mathrm{f}$ en $5 / 6 \mathrm{~d}$ orbitalen, elektronische correlaties en hun rol in de metaal-zuurstofbinding in relatie tot de chemische en fysische eigenschappen.

Samenvattend zullen röntgenexperimenten aan de zuurstof K-edge in de toekomst profiteren van instrumentele ontwikkelingen in resolutie (zowel in ruimte als in tijd). Het grote potentieel van röntgenspectroscopieën in combinatie met de alomtegenwoordigheid van zuurstof in ons universum biedt de mogelijkheid om inzicht te krijgen in de elektronische structuur van veel zuurstofhoudende systemen. Tegelijkertijd zullen theoretische voorspellingen en simulaties de interpretatie van nieuwe gegevens leiden, wat leidt tot nieuwe resultaten van het röntgenonderzoek van zuurstof. 


\section{PUBLICATIONS BY THE AUTHOR}

- Frati F., Hunault M. O., \& de Groot F. M. F. (2020). Oxygen K-edge X-ray absorption spectra. Chemical reviews, 120(9), 4056-4110.

- Frati F., De Groot F. M. F., Cerezo J., Santoro F., Cheng L., Faber R. \& Coriani, S. (2019).Coupled cluster study of the $x$-ray absorption spectra of formaldehyde derivatives at the oxygen, carbon, and fluorine K-edges. The Journal of Chemical Physics, 151(6), 064107.

- Frati F. et al. Density functional study of oxygen Kedge x-ray absorption and electronic structure of alkaline earth metal oxides. (In preparation)

- Bartocci A.,Frati F., Roncaratti L. F., Cappelletti D., Tarantelli F., Belpassi L. \& Pirani F.(2015). An ab initio electronic density study of the $\mathrm{CH}_{4}-\mathrm{Ar}$, $\mathrm{CH}_{4}-\mathrm{Xe}, \mathrm{CH}_{4}-\mathrm{H}_{2} \mathrm{O}$ and $\mathrm{CH}_{4}-\mathrm{H}_{2} \mathrm{~S}$ complexes: insights into the nature of the intermolecular interaction. Molecular Physics, 113(24), 3992-3999.

- Cappelletti D., Bartocci A., Frati F., Roncaratti L. F., Belpassi L., Tarantelli F., Aiswaryalakshmi P, Arunan P. \& Pirani F. (2015). $\mathrm{H}_{2} \mathrm{O}-\mathrm{CH}_{4}$ and $\mathrm{H}_{2} \mathrm{~S}-\mathrm{CH}_{4}$ complexes: a direct comparison through molecular beam experiments and ab initio calculations. Physical Chemistry Chemical Physics, 17(45), 30613-30623.

- Wang R. P., Hariki A., Sotnikov A.,Frati F., Okamoto J., Huang H. Y., Singh A., Huang D.J.,Tomiyasu K., Du C. H., Kuneš J.\& De Groot F. M. F. (2018). Excitonic dispersion of the intermediate spin state in $\mathrm{LaCoO}_{3}$ revealed by resonant inelastic $x$-ray scattering. Physical Review B, 98(3), 035149 .

- Elnaggar H., and Wang R.P., Lafuerza S., Paris E., Komarek A.C.,Guo H.,Tseng Y., McNally D. Frati F.,Haverkort M.W., Sikora M., Schmitt T. \& de Groot F. M. F. (2018). Site selective spin and orbital excitations in $\mathrm{Fe}_{3} \mathrm{O}_{4}$. arXiv preprint arXiv:1811.04836.

- Ismail, A. S., Uemura, Y., Park, S. H., Kwon, S., Kim, M., Elnaggar, H., Frati F., Niwa Y., Wadati H., Hirata Y., Zhang Y., Yamagami K., Yamamoto S., Matsuda I., Halisdemir U., Koster G., Weckhuysen B.M. \& 
De Groot F. M. F.(2020). Direct observation of the electronic states of photoexcited hematite with ultrafast 2p3d X-ray absorption spectroscopy and resonant inelastic $X$-ray scattering. Physical chemistry chemical physics, 22(5), 2685-2692.

- Elnaggar H., Wang R.P., Lafuerza S., Paris E., Komarek A. C., Guo H., Tseng Y., McNally D. Frati F., Haverkort M.W., Sikora M., Schmitt T. \& de Groot F. M. F. (2020). Possible absence of trimeron correlations above the Verwey temperature in $\mathrm{Fe}_{3} \mathrm{O}_{4}$. Physical Review B, 101(8), o85107.

- Wang R. P., Elnaggar H., Titus C. J., Tomiyasu K., Geessinck J., Koster G., Frati F., Okamoto J., Huang D. J. \& de Groot F. M. F. (2020). Saturation and self-absorption effects in the angle-dependent $2 p 3$ d resonant inelastic $X$ ray scattering spectra of $\mathrm{Co}_{3}+$. Journal of Synchrotron Radiation, 27(4).

- Uemura Y.,Ismail A. Park S. H., Kwon S., Kim, M., Niwa Y., Wadati H., Elnaggar H. Frati F., Haarman T., Höppel N., Huse N., Hirata, Y., Zhang Y., Yamagami K., Yamamoto S., Matsuda I., Katayama T., Togashi T., Owada S., Yabashi M., Halisdemir U., Koster G., Yokoyama T., Weckhuysen B., de Groot F. M. F. Femtosecond Charge Density Modulations in Photoexcited $\mathrm{CuWO}_{4}$ (submitted to The Journal of Physical Chemistry)

- F. M. F de Groot, Elnaggar H., Frati F., Wang R. P. et al. $2 p$ x-ray absorption spectroscopy of $3 d$ transition metal systems(In preparation) 
First of all I would like to express my sincere gratitude to Frank for his continuous support, and patience during my PhD study. His deep knowledge and immense kindness have encouraged me in all the time of my academic experience and daily life. I would also like to thank Myrtille for her distant but concrete support. I knew you would have always been available for me and it is a pity we did not have the chance to spend more time working together. During my PhD I had the fortune to learn from excellent researchers around the globe. A very special thanks goes to Sonia Coriani, David Prendergast, Michael Odelius, Hatsushi Hariki and all your collaborators: thank you for welcoming me in your group, I learned a lot from all of you. Thanks to the I21 RIXS group at Diamond for hosting me one month, it has been a short but interesting stay.

I believe the friendly and informal atmosphere at the Utrecht X-Ray group has played a big role in the completion of my $\mathrm{PhD}$, I had the best (and a bit crazy) colleagues I could wish for. A very special thanks goes to Ru-Pan and Heba, we had so many beamtimes together and I had so much fun travelling the world with you. From neverending nights shifts to exotic food experience, I will always remember all the adventures we had. A special mention goes to Marte, again, thanks for the dutch summary!! Marte, you are a lovely and smart lady but please remember: no mint and wine on tomato sauce!!! Manhaz, you will always be an example of elegance I have never seen you loose composure even after 1o hours beamtime shift. Thanks to my favourite smoking buddy, Ties: one day I will may come to climb with you and Daria. Ahmed, I will never forget how hard you tried to find me a cigarette during my first night shift at Soleil, in Paris. I really appreciated your effort. My favourite (very) chinese colleague will always be Boyang, I finally found in you a real pork tripes estimator. Thank Yohei, I really enjoyed your exotic lunch discussions and your "surrealistic" questions. Last but not least thank to Patrik, Matti and Mario for the privacy-related and scientific advices. I am really greateful and honored to had the chance to work with all of you guys. Despite the isolation of the X-Ray group from big ICC group, I found some great people that I cannot forget to thank. Marisol, you are the shoulder to lean on everybody needs, I am so glad we meet each other. Can't wait for next mexican/italian/indian dinner. Thanks to the gentleman Jose' and 
many others for our enjoyable coffe breaks: your smile and your kind words will never be forgotten. I was lucky enough to tutor Hanya during my last year of PhD during her bachelor thesis, I wish you all the best for your future career. A special mention goes to the technical and administrative staff of ICC, thanks for helping me in every issue I had.

Un ringraziamento del tutto speciale va ai miei genitori. I ringraziamenti alla fine di questa tesi sono solo una goccia nell'oceano di riconoscenza che provo nei vostri confronti. Grazie per la guida costante, il supporto incondizionato e l'amore tangibile che in questi 28 anni non sono mai venuti a meno. Grazie a mia sorella biologica Stefania e mia sorella di cuore Anastasia, non ci sono parole per descrivervi (o forse é meglio non cercarle!),grazie per essere sempre state al mio fianco nonostante i $1500 \mathrm{~km}$ che ci separano. Grazie ai miei uomini, Simone ed Edoardo, avete reso la mia vita una bellissima avventura e con voi accanto tutte le difficoltá non sono poi cosí insormontabili. 
I was born in Umbertide, Italy, on August 23,1992. I obtained my high school diploma at the Campus Leonardo da Vinci Umbertide specializing in linguistic study in 2011. I enrolled in the bachelor program Chemistry at the University of Perugia and I obtained my bachelor degree in 2014 with the thesis "The $\mathrm{CH}_{4}-\mathrm{H}_{2} \mathrm{O}$ complex: molecular beam scattering experiments and ab initio calculations" under the supervision of Prof. F. Tarantelli and Dott. L. Belpassi. In the same year I moved to Trieste where I enrolled for the master in Chemistry at the University of Trieste with the TCCM (Theoretical Chemistry and Computational Modelling) program. After spending six months at the University of Groningen, I obtained the master degree with the thesis "Density Functional Study of CdSe, CdS and CdTe Quantum Dots" under the supervision of Prof. R.W.A. Havenith and Prof. M. Stener. In August 2016 I started my PhD in the group of Prof. F.M.F de Groot at the Debye Institute for Nanomaterials Science at Utrecht University. 

[1] W Kossel. "Zum bau der röntgenspektren." In: Z. Phys. A: Hadrons Nucl. 1.1 (1920), pp. 119-134.

[2] R Stumm Von Bordwehr. "A history of X-ray absorption fine structure." In: Ann. Phys. Vol. 14. 4. EDP Sciences. 1989, pp. 377-465.

[3] John Emsley. Nature's building blocks: an A-Z guide to the elements. en. Oxford University Press, 2001. IsBN: 978-0-19-850341-5.

[4] James E House and Kathleen A House. Descriptive inorganic chemistry. Academic Press, 2015.

[5] Tod A Pascal, Ulrike Boesenberg, Robert Kostecki, Thomas J Richardson, Tsu-Chien Weng, Dimosthenis Sokaras, Dennis Nordlund, Eamon McDermott, Alexander Moewes, and Jordi Cabana. "Finite temperature effects on the X-ray absorption spectra of lithium compounds: First-principles interpretation of X-ray Raman measurements." In: J. Chem. Phys. 140.3 (2014), p. 034107.

[6] C Kolczewski, R Püttner, O Plashkevych, Hans Ågren, V Staemmler, M Martins, G Snell, AS Schlachter, M Sant'Anna, and G Kaindl. "Detailed study of pyridine at the $\mathrm{C}$ is and $\mathrm{N}$ is ionization thresholds: The influence of the vibrational fine structure." In: J. Chem. Phys. 115.14 (2001), pp. 6426-6437.

[7] Andreas Görling. "Density-functional theory beyond the HohenbergKohn theorem." In: Phys. Rev. A 59.5 (1999), p. 3359.

[8] Jeppe Olsen, Michel R Godefroid, Per Jönsson, Per Åke Malmqvist, and Charlotte Froese Fischer. "Transition probability calculations for atoms using nonorthogonal orbitals." In: Phys. Rev. E 52.4 (1995), p. 4499 .

[9] L Triguero, O Plashkevych, LGM Pettersson, and H Ågren. "Separate state vs. transition state Kohn-Sham calculations of X-ray photoelectron binding energies and chemical shifts." In: Journal of electron spectroscopy and related phenomena 104.1-3 (1999), pp. 195-207.

[10] Nicholas A Besley, Andrew TB Gilbert, and Peter MW Gill. "Selfconsistent-field calculations of core excited states." In: J. Chem. Phys. 130.12 (2009), p. 124308. 
[11] Andreas Dreuw, Jennifer L Weisman, and Martin Head-Gordon. "Longrange charge-transfer excited states in time-dependent density functional theory require non-local exchange." In: The Journal of chemical physics 119.6 (2003), pp. 2943-2946.

[12] John C Slater and Keith H Johnson. "Self-consistent-field X $\alpha$ cluster method for polyatomic molecules and solids." In: Phys. Rev. B 5.3 (1972), p. 844 .

[13] Mikael Leetmaa, MP Ljungberg, Alexander Lyubartsev, Anders Nilsson, and Lars GM Pettersson. "Theoretical approximations to X-ray absorption spectroscopy of liquid water and ice." In: J. Electron Spectrosc. Relat. Phenom. 177.2 (2010), pp. 135-157.

[14] David Prendergast and Giulia Galli. "X-ray absorption spectra of water from first principles calculations." In: Physical review letters 96.21 (2006), p. 215502.

[15] Nicolas Ferré, Michael Filatov, Miquel Huix-Rotllant, and C Adamo. Density-functional methods for excited states. Springer, 2016.

[16] L Triguero, LGM Pettersson, and H Ågren. “Calculations of nearedge $\mathrm{x}$-ray-absorption spectra of gas-phase and chemisorbed molecules by means of density-functional and transition-potential theory." In: Phys. Rev. B 58.12 (1998), p. 8097.

[17] Mats Nyberg, Yi Luo, Luciano Triguero, Lars GM Pettersson, and Hans Ågren. "Core-hole effects in X-ray-absorption spectra of fullerenes." In: Phys. Rev. B 60.11 (1999), p. 7956.

[18] Shang-Di Mo and WY Ching. "Ab initio calculation of the core-hole effect in the electron energy-loss near-edge structure." In: Phys. Rev. B 62.12 (2000), p. 7901.

[19] WH Eugen Schwarz. "X-Ray Absorption Spectroscopy on Free Molecules." In: Angewandte Chemie International Edition in English 13.7 (1974), pp. 454465 .

[20] WHE Schwarz and Robert J Buenker. "Use of the Z+ 1-core analogy model: examples from the core-excitation spectra of $\mathrm{CO}_{2}$ and $\mathrm{N}_{2} \mathrm{O}$." In: Chemical Physics 13.2 (1976), pp. 153-160.

[21] Matteo Cavalleri, Michael Odelius, Dennis Nordlund, Anders Nilsson, and Lars GM Pettersson. "Half or full core hole in density functional theory X-ray absorption spectrum calculations of water?" In: Phys. Chem. Chem. Phys. 7.15 (2005), pp. 2854-2858. 
[22] E Chanet, M De Crescenzi, and J Derrien. "Validity of the $(Z+1)$ ioncore approximation for deep and shallow levels as studied by the extended energy-loss fine-structure technique." In: Phys. Rev. B 31.11 (1985), p. 7469.

[23] John J Rehr, Joshua J Kas, Fernando D Vila, Micah P Prange, and Kevin Jorissen. "Parameter-free calculations of X-ray spectra with FEFF9." In: Phys. Chem. Chem. Phys. 12.21 (2010), pp. 5503-5513.

[24] Karlheinz Schwarz, Peter Blaha, and Georg KH Madsen. "Electronic structure calculations of solids using the WIEN2k package for material sciences." In: Comput. Phys. Commun. 147.1-2 (2002), pp. 71-76.

[25] Paolo Giannozzi, Oliviero Andreussi, Thomas Brumme, Oana Bunau, M Buongiorno Nardelli, Matteo Calandra, Roberto Car, Carlo Cavazzoni, Davide Ceresoli, and Matteo Cococcioni. "Advanced capabilities for materials modelling with Quantum ESPRESSO." In: J. Phys.: Condens. Matter 29.46 (2017), p. 465901.

[26] G t Te Velde, F Matthias Bickelhaupt, Evert Jan Baerends, C Fonseca Guerra, Stan JA van Gisbergen, Jaap G Snijders, and Tom Ziegler. "Chemistry with ADF." In: J. Comput. Chem. 22.9 (2001), pp. 931-967.

[27] Frank Neese. "The ORCA program system." In: Wiley Interdisciplinary Reviews: Computational Molecular Science 2.1 (2012), pp. 73-78.

[28] O Krogh Andersen. "Linear methods in band theory." In: Phys. Rev. B 12.8 (1975), p. 3060.

[29] James R Chelikowsky, N Troullier, and Y Saad. "Finite-differencepseudopotential method: Electronic structure calculations without a basis." In: Phys. Rev. Lett. 72.8 (1994), p. 1240.

[30] Shang-Peng Gao, Chris J Pickard, Mike C Payne, Jing Zhu, and Jun Yuan. "Theory of core-hole effects in is core-level spectroscopy of the first-row elements." In: Phys. Rev. B 77.11 (2008), p. 115122.

[31] Delphine Cabaret, Francesco Mauri, and Grant S Henderson. “Oxygen K-edge XANES of germanates investigated using first-principles calculations." In: Phys. Rev. B 75.18 (2007), p. 184205.

[32] Delphine Cabaret, Emilie Gaudry, Mathieu Taillefumier, Philippe Sainctavit, and Francesco Mauri. "XANES calculation with an efficient "non muffin-tin" method: application to the angular dependence of the Al K-edge in corundum." In: Phys. Scr. 2005.T115 (2005), p. 131.

[33] Nan Jiang. "Structure and composition dependence of oxygen K edge in $\mathrm{CaAl}_{2} \mathrm{O}_{4}$." In: J. Appl. Phys. 100.1 (2006), p. 013703. 
[34] A. Zangwill and Paul Soven. "Density-functional approach to localfield effects in finite systems: photoabsorption in the rare gases." In: Phys. Rev. A 21 (5 1980), pp. 1561-1572.

[35] J Zaanen, GA Sawatzky, J Fink, W Speier, and JC Fuggle. “L2,3 absorption spectra of the lighter $3 \mathrm{~d}$ transition metals." In: Phys. Rev. B 32.8 (1985), p. 4905.

[36] J Vinson, JJ Rehr, JJ Kas, and EL Shirley. "Bethe-Salpeter equation calculations of core excitation spectra." In: Phys. Rev. B 83.11 (2011), p. 115106.

[37] Oana Bunău and Yves Joly. "Time-dependent density functional theory applied to x-ray absorption spectroscopy." In: Phys. Rev. B 85.15 (2012), p. 155121.

[38] Erich Runge and E. K. U. Gross. "Density-functional theory for timedependent systems." In: Phys. Rev. Lett. 52 (12 1984), pp. 997-1000.

[39] Giovanni Onida, Lucia Reining, and Angel Rubio. “Electronic excitations: density-functional versus many-body Green's-function approaches." In: Rev. Mod. Phys. 74.2 (2002), p. 601.

[40] Miguel AL Marques and Eberhard KU Gross. "Time-dependent density functional theory." In: Annu. Rev. Phys. Chem. 55 (2004), pp. 427455.

[41] Lucia Reining, Valerio Olevano, Angel Rubio, and Giovanni Onida. "Excitonic effects in solids described by time-dependent density-functional theory." In: Phys. Rev. Lett. 88.6 (2002), p. 066404.

[42] Yong-Hoon Kim and Andreas Görling. "Excitonic optical spectrum of semiconductors obtained by time-dependent density-functional theory with the exact-exchange kernel." In: Phys. Rev. Lett. 89.9 (2002), p. 096402.

[43] George F Bertsch, J-I Iwata, Angel Rubio, and Kazuhiro Yabana. "Realspace, real-time method for the dielectric function." In: Phys. Rev. B 62.12 (2000), p. 7998.

[44] J Vinson, JJ Kas, FD Vila, JJ Rehr, and EL Shirley. "Theoretical optical and X-ray spectra of liquid and solid $\mathrm{H}_{2}$ O." In: Phys. Rev. B 85.4 (2012), p. 045101.

[45] Keith Gilmore, John Vinson, Eric L Shirley, David Prendergast, C Das Pemmaraju, Joshua J Kas, Fernando D Vila, and John J Rehr. "Efficient implementation of core-excitation Bethe-Salpeter equation calculations." In: Comput. Phys. Commun. 197 (2015), pp. 109-117. 
[46] Tim Zuehlsdorff. Computing the optical properties of large systems. Springer, 2015.

[47] J Van Elp and Arata Tanaka. "Threshold electronic structure at the oxygen K edge of 3 d-transition-metal oxides: A configuration interaction approach." In: Phys. Rev. B 60.8 (1999), p. 5331.

[48] Frank De Groot and Akio Kotani. Core level spectroscopy of solids. CRC press, 2008.

[49] WHE Schwarz and TC Chang. "Multiconfiguration wave functions for highly excited states by the generalized Brillouin theorem method." In: Int. J. Quantum Chem. 10.Sio (1976), pp. 91-97.

[50] Francesco Antonio Gianturco and Maurizio Zandomeneghi. "External constraints for inner-shell molecular ion calculations." In: J. Chim. Phys. Phys.-Chim. Biol. 71 (1974), pp. 18-20.

[51] Sonia Coriani, Ove Christiansen, Thomas Fransson, and Patrick Norman. "Coupled-cluster response theory for near-edge x-ray-absorption fine structure of atoms and molecules." In: Phys. Rev. A 85.2 (2012), p. 022507.

[52] Marcel Nooijen and Rodney J Bartlett. "Description of core-excitation spectra by the open-shell electron-attachment equation-of-motion coupled cluster method." In: J. Chem. Phys. 102.17 (1995), pp. 6735-6756.

[53] Frans A Asmuruf and Nicholas A Besley. "Calculation of near-edge X-ray absorption fine structure with the CIS (D) method." In: Chem. Phys. Lett. 463.1-3 (2008), pp. 267-271.

[54] Kei Kuramoto, Masahiro Ehara, and Hiroshi Nakatsuji. "Theoretical fine spectroscopy with symmetry adapted cluster-configuration interaction general-R method: First-row K-shell ionizations and their satellites." In: J. Chem. Phys. 122.1 (2005), p. 014304.

[55] Alexandre B Rocha. "Potential curves for inner-shell states of CO calculated at multiconfigurational self-consistent field level." In: J. Chem. Phys. 134.2 (2011), p. 024107.

[56] Thomas Fransson, Yoshihisa Harada, Nobuhiro Kosugi, Nicholas A Besley, Bernd Winter, John J Rehr, Lars GM Pettersson, and Anders Nilsson. "X-ray and electron spectroscopy of water." In: Chem. Rev. 116.13 (2016), pp. 7551-7569.

[57] David W Fischer. Soft X-ray band spectra and molecular orbital structure of $\mathrm{Cr}_{2} \mathrm{O}_{3}, \mathrm{CrO}_{3}, \mathrm{CrO}_{4}^{-2}$, and $\mathrm{Cr}_{2} \mathrm{O}_{7}^{-2}$. Tech. rep. AIR FORCE MATERIALS LAB WRIGHT-PATTERSON AFB OH, 1971. 
[58] J Stöhr, R Jaeger, J Feldhaus, S Brennan, D Norman, and G Apai. “Extended absorption fine structure studies above the carbon, nitrogen, oxygen, and fluorine K absorption edges." In: Appl. Opt. 19.23 (1980), pp. 3911-3919.

[59] Helmuth Petersen. "The high energy plane grating monochromators at BESSY." In: Nucl. Instrum. Methods Phys. Res., Sect. A 246.1-3 (1986), pp. 260-263.

[6o] CT Chen and F Sette. "Performance of the Dragon soft x-ray beamline." In: Rev. Sci. Instrum. 60.7 (1989), pp. 1616-1621.

[61] Matthias Müller, Tobias Mey, Jürgen Niemeyer, and Klaus Mann. "Table-top soft x-ray microscope using laser-induced plasma from a pulsed gas jet." In: Opt. Express 22.19 (2014), pp. 23489-23495.

[62] Frank-Christian Kühl, Matthias Müller, Meike Schellhorn, Klaus Mann, Stefan Wieneke, and Karin Eusterhues. "Near-edge x-ray absorption fine structure spectroscopy at atmospheric pressure with a table-top laser-induced soft x-ray source." In: J. Vac. Sci. Technol., A 34.4 (2016), p. 041302.

[63] SM Teichmann, F Silva, SL Cousin, M Hemmer, and J Biegert. "o.5keV Soft X-ray attosecond continua." In: Nat. Commun. 7 (2016), p. 11493.

[64] Seth L Cousin, Nicola Di Palo, Bárbara Buades, Stephan M Teichmann, M Reduzzi, M Devetta, A Kheifets, G Sansone, and Jens Biegert. "Attosecond streaking in the water window: A new regime of attosecond pulse characterization." In: Phys. Rev. X 7.4 (2017), p. o41ozo.

[65] Cédric Schmidt, Yoann Pertot, Tadas Balciunas, Kristina Zinchenko, Mary Matthews, Hans Jakob Wörner, and Jean-Pierre Wolf. "Highorder harmonic source spanning up to the oxygen K-edge based on filamentation pulse compression." In: Opt. Express 26.9 (2018), pp. 1183411842 .

[66] Carlo Kleine, Maria Ekimova, Gildas Goldsztejn, Sebastian Raabe, Christian Strüber, Jan Ludwig, Suresh Yarlagadda, Stefan Eisebitt, Marc JJ Vrakking, and Thomas Elsaesser. "Soft X-ray absorption spectroscopy of aqueous solutions using a table-top femtosecond soft $\mathrm{X}$ ray source." In: J. Phys. Chem. Lett. (2018).

[67] Allan S Johnson, Dane R Austin, David A Wood, Christian Brahms, Andrew Gregory, Konstantin B Holzner, Sebastian Jarosch, Esben W Larsen, Susan Parker, and Christian S Strüber. "High-flux soft x-ray 
harmonic generation from ionization-shaped few-cycle laser pulses." In: Sci. Adv. 4.5 (2018), eaar3761.

[68] Cor P de Vries, JW Den Herder, JS Kaastra, FB Paerels, AJ Den Boggende, and AP Rasmussen. "The interstellar oxygen-K absorption edge as observed by XMM-Newton-Separation of instrumental and interstellar components." In: Astron. Astrophys. 404.3 (2003), pp. 959-967.

[69] J Jaklevic, JA Kirby, MP Klein, AS Robertson, GS Brown, and P Eisenberger. "Fluorescence detection of exafs: Sensitivity enhancement for dilute species and thin films." In: Solid State Commun. 23.9 (1977), pp. 679-682.

[70] L Tröger, D Arvanitis, K Baberschke, H Michaelis, U Grimm, and E Zschech. "Full correction of the self-absorption in soft-fluorescence extended X-ray-absorption fine structure." In: Phys. Rev. B 46.6 (1992), p. 3283 .

[71] S Eisebitt, T Böske, J-E Rubensson, and Wolfgang Eberhardt. "Determination of absorption coefficients for concentrated samples by fluorescence detection." In: Phys. Rev. B 47.21 (1993), p. 14103.

[72] RJ Green, D Peak, AJ Achkar, JS Tse, A Moewes, DG Hawthorn, and TZ Regier. "Comment on "state-dependent electron delocalization dynamics at the solute-solvent interface: soft-X-ray absorption spectroscopy and ab initio calculations"." In: Phys. Rev. Lett. 112.12 (2014), p. 129301.

[73] FMF De Groot, MA Arrio, Ph Sainctavit, Ch Cartier, and CT Chen. "Fluorescence yield detection: Why it does not measure the X-ray absorption cross section." In: Solid State Commun. 92.12 (1994), pp. 991995 .

[74] Frank MF De Groot. "Dips and peaks in fluorescence yield X-ray absorption are due to state-dependent decay." In: Nat. Chem. 4.10 (2012), p. 766 .

[75] Alexander Foehlisch, FMF de Groot, M Odelius, Simone Techert, and $\mathrm{Ph}$ Wernet. "Comment on "state-dependent electron delocalization dynamics at the solute-solvent interface: soft-X-ray absorption spectroscopy and ab initio calculations"." In: Phys. Rev. Lett. 112.12 (2014), p. 129302.

[76] Mauro Rovezzi, Christophe Lapras, Alain Manceau, Pieter Glatzel, and Roberto Verbeni. "High energy-resolution x-ray spectroscopy at 
ultra-high dilution with spherically bent crystal analyzers of $0.5 \mathrm{~m}$ radius." In: Rev. Sci. Instrum. 88.1 (2017), p. 013108.

[77] AJ Achkar, TZ Regier, H Wadati, Y-J Kim, H Zhang, and DG Hawthorn. "Bulk sensitive x-ray absorption spectroscopy free of self-absorption effects." In: Phys. Rev. B 83.8 (2011), p. 081106.

[78] Wolfgang Gudat and Christof Kunz. "Close similarity between photoelectric yield and photoabsorption spectra in the soft-x-ray range." In: Phys. Rev. Lett. 29.3 (1972), p. 169.

[79] Reiko Nakajima, J Stöhr, and Yves U Idzerda. “Electron-yield saturation effects in L-edge $\mathrm{x}$-ray magnetic circular dichroism spectra of $\mathrm{Fe}$, Co, and Ni." In: Phys. Rev. B 59.9 (1999), p. 6421.

[8o] Jan Vogel and Maurizio Sacchi. "Experimental estimate of absorption length and total electron yield (TEY) probing depth in dysprosium." In: J. Electron Spectrosc. Relat. Phenom. 67.1 (1994), pp. 181-188.

[81] SLM Schroeder, GD Moggridge, RM Ormerod, T Rayment, and RM Lambert. "What determines the probing depth of electron yield XAS?" In: Surf. Sci. 324.2-3 (1995), pp. L371-L377.

[82] Axel Knop-Gericke, Michael Hävecker, Th Schedel-Niedrig, and Robert Schlögl. "High-pressure low-energy XAS: a new tool for probing reacting surfaces of heterogeneous catalysts." In: Topics in Catalysis 10.3 (2000), pp. 187-198.

[83] Daniela Schon, Jie Xiao, Ronny Golnak, Marc F Tesch, Bernd Winter, Juan-Jesus Velasco-Velez, and Emad F Aziz. "Introducing ioniccurrent detection for X-Ray absorption spectroscopy in liquid cells." In: J. Phys. Chem. Lett. 8.9 (2017), pp. 2087-2092.

[84] FJ Himpsel, Ulf O Karlsson, AB McLean, LJ Terminello, FMF De Groot, M Abbate, JC Fuggle, JA Yarmoff, BT Thole, and GA Sawatzky. "Fine structure of the Ca 2p x-ray-absorption edge for bulk compounds, surfaces, and interfaces." In: Phys. Rev. B 43.9 (1991), p. 6899.

[85] Wayne C Stolte, MM Sant'Anna, Gunnar Öhrwall, Ivan DominguezLopez, MN Piancastelli, and Dennis W Lindle. "Photofragmentation dynamics of core-excited water by anion-yield spectroscopy." In: Phys. Rev. A 68.2 (2003), p. 022701.

[86] Tetsuichiro Hayakawa, Masashi Arakawa, Shun Sarugaku, Kota Ando, Kenichirou Tobita, Yuya Kiyomura, Tomoki Kawano, and Akira Terasaki. "Characterization of Cerium and oxygen atoms in free clusters of 
cerium oxide by X-ray absorption spectroscopy." In: Top. Catal. 61.1-2 (2018), pp. 119-125.

[87] T Pylkkanen, J Lehtola, M Hakala, A Sakko, G Monaco, S Huotari, and $\mathrm{K}$ Hamalainen. "Universal signature of hydrogen bonding in the oxygen K-edge spectrum of alcohols." In: J. Phys. Chem. B 114.41 (2010), pp. 13076-13083.

[88] C Sternemann and M Wilke. "Spectroscopy of low and intermediate $\mathrm{Z}$ elements at extreme conditions: in situ studies of Earth materials at pressure and temperature via X-ray Raman scattering." In: High Pressure Res. 36.3 (2016), pp. 275-292.

[89] Uwe Bergmann, Andrea Di Cicco, Philippe Wernet, Emiliano Principi, Pieter Glatzel, and Anders Nilsson. "Nearest-neighbor oxygen distances in liquid water and ice observed by x-ray Raman based extended x-ray absorption fine structure." In: J. Chem. Phys. 127.17 (2007), p. 174504.

[9o] Benjamin JA Moulton, Grant S Henderson, Hiroshi Fukui, Nozomu Hiraoka, Dominique de Ligny, Camille Sonneville, and Masami Kanzaki. "In situ structural changes of amorphous diopside $\left(\mathrm{CaMgSi}_{2} \mathrm{O}_{6}\right)$ up to $20 \mathrm{GPa}$ : A Raman and O K-edge X-ray Raman spectroscopic study." In: Geochim. Cosmochim. Acta 178 (2016), pp. 41-61.

[91] J Fink, Th Müller-Heinzerling, B Scheerer, W Speier, FU Hillebrecht, JC Fuggle, J Zaanen, and GA Sawatzky. "2p absorption spectra of the 3d elements." In: Phys. Rev. B 32.8 (1985), p. 4899.

[92] C Colliex, T Manoubi, and C Ortiz. "Electron-energy-loss-spectroscopy near-edge fine structures in the iron-oxygen system." In: Phys. Rev. B 44.20 (1991), p. 11402.

[93] A Gorschlüter and H Merz. "Resonant electron exchange scattering in late transition metal monoxides." In: J. Electron Spectrosc. Relat. Phenom. 87.3 (1998), pp. 211-220.

[94] Bärbel Fromme, M Möller, C Bethke, U Brunokowski, and E Kisker. "Resonant electron-exchange excitations in transition-metal oxides." In: Phys. Rev. B 57.19 (1998), p. 12069.

[95] DA Fischer, JL Gland, and SM Davis. "Fluorescence yield near edge spectroscopy (FYNES) of benzene adsorbed in Na-Y zeolite." In: Catal. Lett. 6.1 (1990), pp. 99-102. 
[96] Emiel de Smit, Ingmar Swart, J Fredrik Creemer, Gerard H Hoveling, Mary K Gilles, Tolek Tyliszczak, Patricia J Kooyman, Henny W Zandbergen, Cynthia Morin, Bert M Weckhuysen, et al. "Nanoscale chemical imaging of a working catalyst by scanning transmission $\mathrm{X}$ ray microscopy." In: Nature 456.7219 (2008), p. 222.

[97] Sharadha Sambasivan, Daniel A Fischer, Benjamin M DeKoven, and Alex Kuperman. "Direct observations of propylene and silver transformations on the surface and in the pores of silver $Y$ zeolites." In: Adv. Mater. 12.23 (2000), pp. 1809-1813.

[98] Axel Knop-Gericke, Michael Hävecker, Thomas Schedel-Niedrig, and Robert Schlögl. "Characterisation of active phases of a copper catalyst for methanol oxidation under reaction conditions: an in situ X-ray absorption spectroscopy study in the soft energy range." In: Top. Catal. 15.1 (2001), pp. 27-34.

[99] M Hävecker, A Knop-Gericke, and Th Schedel-Niedrig. "High-pressure soft X-ray absorption spectroscopy: application of a new in situ spectroscopic method in catalysis research." In: App. Surf. Sci. 142.1-4 (1999), pp. 438-442.

[100] WM Heijboer, Andrea A Battiston, Axel Knop-Gericke, Michael Hävecker, Hendrik Bluhm, Bert M Weckhuysen, Diek C Koningsberger, and Frank MF de Groot. "Redox behaviour of over-exchanged Fe/ZSM5 zeolites studied with in-situ soft X-ray absorption spectroscopy." In: Phys. Chem. Chem. Phys. 5.20 (2003), pp. 4484-4491.

[101] Fernando Morales, Frank MF de Groot, Pieter Glatzel, Evgueni Kleimenov, Hendrik Bluhm, Michael Haevecker, Axel Knop-Gericke, and Bert $\mathrm{M}$ Weckhuysen. "In situ X-ray absorption of $\mathrm{Co} / \mathrm{Mn} / \mathrm{TiO}_{2}$ catalysts for Fischer- Tropsch synthesis." In: J. Phys. Chem. B 108.41 (2004), pp. 16201-16207.

[102] DJ Miller, Henrik Öberg, S Kaya, H Sanchez Casalongue, D Friebel, T Anniyev, H Ogasawara, H Bluhm, Lars GM Pettersson, and Anders Nilsson. "Oxidation of Pt (111) under near-ambient conditions." In: Phys. Rev. Lett. 107.19 (2011), p. 195502.

[103] Michael Hävecker, Axel Knop-Gericke, Ralf W Mayer, Martin Fait, Hendrik Bluhm, and Robert Schlögl. "Influence of the geometric structure on the $\mathrm{V} \mathrm{L}_{3}$ near edge $\mathrm{X}$-ray absorption fine structure from vanadium phosphorus oxide catalysts." In: J. Electron Spectrosc. Relat. Phenom. 125.2 (2002), pp. 79-87. 
[104] Michael Hävecker, Matteo Cavalleri, Rita Herbert, Rolf Follath, Axel Knop-Gericke, Christian Hess, Klaus Hermann, and Robert Schlögl. "Methodology for the structural characterisation of VxOy species supported on silica under reaction conditions by means of in situ O Kedge X-ray absorption spectroscopy." In: Phys. Status Solidi B 246.7 (2009), pp. 1459-1469.

[105] C Castán-Guerrero, D Krizmancic, V Bonanni, R Edla, A Deluisa, F Salvador, G Rossi, G Panaccione, and P Torelli. "A reaction cell for ambient pressure soft x-ray absorption spectroscopy." In: Rev. Sci. Instrum. 89.5 (2018), p. 054101.

[106] A Cavalleri, M Rini, HHW Chong, S Fourmaux, TE Glover, PA Heimann, JC Kieffer, and RW Schoenlein. "Band-selective measurements of electron dynamics in $\mathrm{VO}_{2}$ using femtosecond near-edge X-ray absorption." In: Phys. Rev. Lett. 95.6 (2005), p. 067405.

[107] TJA Wolf, Rolf H Myhre, JP Cryan, Sonia Coriani, RJ Squibb, Andrea Battistoni, Nora Berrah, Christoph Bostedt, Phil Bucksbaum, and Giacomo Coslovich. "Probing ultrafast $\pi \pi^{*} / \mathrm{n} \pi^{*}$ internal conversion in organic chromophores via K-edge resonant absorption." In: Nat. Commun. 8.1 (2017), p. 29.

[108] Martin Beye, Toyli Anniyev, R Coffee, Martina DellAngela, Alexander Foehlisch, Jörgen Gladh, T Katayama, S Kaya, O Krupin, and Andreas Møgelhøj. "Selective ultrafast probing of transient hot chemisorbed and precursor states of CO on Ru (ooo1)." In: Phys. Rev. Lett. 110.18 (2013), p. 186101.

[109] Martin Beye, Henrik Oberg, Hongliang Xin, Georgi L Dakovski, Martina Dell'Angela, Alexander Fohlisch, Jorgen Gladh, Markus Hantschmann, Florian Hieke, and Sarp Kaya. "Chemical bond activation observed with an x-ray laser." In: J. Phys. Chem. Lett. 7.18 (2016), pp. 3647-3651.

[110] Jonas A Sellberg, Trevor A McQueen, Hartawan Laksmono, Simon Schreck, Martin Beye, Daniel P DePonte, Brian Kennedy, Dennis Nordlund, Raymond G Sierra, and Daniel Schlesinger. "X-ray emission spectroscopy of bulk liquid water in "no-man's land"." In: J. Chem. Phys. 142.4 (2015), p. 044505.

[111] Simon Schreck, Martin Beye, Jonas A Sellberg, Trevor McQueen, Hartawan Laksmono, Brian Kennedy, Sebastian Eckert, Daniel Schlesinger, Dennis Nordlund, Hirohito Ogasawara, et al. "Reabsorption of soft x-ray emission at high x-ray free-electron laser fluences." In: Phys. Rev. Lett. 113.15 (2014), p. 153002. 
[112] DA Muller, L Fitting Kourkoutis, M Murfitt, JH Song, HY Hwang, J Silcox, N Dellby, and OL Krivanek. "Atomic-scale chemical imaging of composition and bonding by aberration-corrected microscopy." In: Science 319.5866 (2008), pp. 1073-1076.

[113] J Verbeeck, S Bals, AN Kravtsova, D Lamoen, M Luysberg, Mark Huijben, G Rijnders, Alexander Brinkman, H Hilgenkamp, and David HA Blank. "Electronic reconstruction at n-type $\mathrm{SrTiO}_{3} / \mathrm{LaAlO}_{3}$ interfaces." In: Phys. Rev. B 81.8 (2010), p. o85113.

[114] David A Muller, T Sorsch, S Moccio, FH Baumann, K Evans-Lutterodt, and G Timp. "The electronic structure at the atomic scale of ultrathin gate oxides." In: Nature 399.6738 (1999), p. 758.

[115] R Egoavil, S Hühn, M Jungbauer, N Gauquelin, A Béché, G Van Tendeloo, J Verbeeck, and V Moshnyaga. "Phase problem in the B-site ordering of $\mathrm{La}_{2} \mathrm{CoMnO}_{6}$ : impact on structure and magnetism." In: Nanoscale 7.21 (2015), pp. 9835-9843.

[116] Dan Zhou, Wilfried Sigle, Eiji Okunishi, Yi Wang, Marion Kelsch, Hanns-Ulrich Habermeier, and Peter A van Aken. "Atomic-resolution TEM studies of Pillar-Matrix structures in epitaxially grown ultrathin $\mathrm{ZrO}_{2}-\mathrm{La}_{2 / 3} \mathrm{Sr}_{1 / 3} \mathrm{MnO}_{3}$ films." In: arXiv preprint arXiv:1409.1172 (2014).

[117] Yi Wang, Michael RS Huang, Ute Salzberger, Kersten Hahn, Wilfried Sigle, and Peter A van Aken. "Towards atomically resolved EELS elemental and fine structure mapping via multi-frame and energy-offset correction spectroscopy." In: Ultramicroscopy 184 (2018), pp. 98-105.

[118] Frank MF de Groot, Emiel de Smit, Matti M van Schooneveld, Luis R Aramburo, and Bert M Weckhuysen. "In-situ scanning transmission X-ray microscopy of catalytic solids and related nanomaterials." In: Chem. Phys. Chem. 11.5 (2010), pp. 951-962.

[119] Aditya Sharma, Mayora Varshney, Sitansu Sekhar Nanda, Hyun Joon Shin, Namdong Kim, Dong Kee Yi, Keun-Hwa Chae, and Sung Ok Won. "Structural, electronic structure and antibacterial properties of graphene-oxide nano-sheets." In: Chem. Phys. Lett. 698 (2018), pp. 8592.

[120] Jesse D Ward, Mark Bowden, C Tom Resch, Steven Smith, Bruce K McNamara, Edgar C Buck, Gregory C Eiden, and Andrew M Duffin. "Identification of uranyl minerals using oxygen K-edge X-ray absorption spectroscopy." In: Geostand. Geoanal. Res. 40.1 (2016), pp. 135-148. 
[121] Alison B Altman, Joseph I Pacold, Jian Wang, Wayne W Lukens, and Stefan G Minasian. "Evidence for $5 \mathrm{~d}-\sigma$ and $5 \mathrm{~d}-\pi$ covalency in lanthanide sesquioxides from oxygen K-edge X-ray absorption spectroscopy." In: Dalton Trans. 45.24 (2016), pp. 9948-9961.

[122] Toyohiko Kinoshita, Takanori Wakita, HaiLin Sun, Takahide Tohyama, Ayumi Harasawa, Hideyuki Kiwata, F Ulrich Hillebrecht, Kanta Ono, Takeshi Matsushima, and Masaharu Oshima. "Antiferromagnetic domain structure imaging of cleaved $\mathrm{NiO}(100)$ surface using nonmagnetic linear dichroism at $\mathrm{O} \mathrm{K}$ edge: essential effect of antiferromagnetic crystal distortion." In: J. Phys. Soc. Jpn. 73.11 (2004), pp. 29322935.

[123] C Morin, AP Hitchcock, RM Cornelius, JL Brash, SG Urquhart, A Scholl, and A Doran. "Selective adsorption of protein on polymer surfaces studied by soft X-ray photoemission electron microscopy." In: J. Electron Spectrosc. Relat. Phenom. 137 (2004), pp. 785-794.

[124] Alexander Bagger, Ties Haarman, Anna Puig Molina, Poul George Moses, Hirofumi Ishii, Nozomu Hiraoka, Y-H Wu, K-D Tsuei, Ib Chorkendorff, and Frank De Groot. "Is2p resonant inelastic X-ray scattering combined dipole and quadrupole analysis method." In: J. Synchrotron Radiat. 24.1 (2017), pp. 296-301.

[125] M Moretti Sala, M Rossi, S Boseggia, J Akimitsu, NB Brookes, M Isobe, Matteo Minola, H Okabe, Henrik M Rønnow, and L Simonelli. "Orbital occupancies and the putative $\mathrm{j}$ eff $=12$ ground state in $\mathrm{Ba}_{2} \mathrm{IrO}_{4}$ : A combined oxygen K-edge XAS and RIXS study." In: Phys. Rev. B 89.12 (2014), p. 121101.

[126] X Liu, MPM Dean, J Liu, SG Chiuzbăian, N Jaouen, A Nicolaou, WG Yin, C Rayan Serrao, R Ramesh, and H Ding. "Probing single magnon excitations in $\mathrm{Sr}_{2} \mathrm{IrO}_{4}$ using $\mathrm{O}$ K-edge resonant inelastic x-ray scattering." In: J. Phys.: Condens. Matter 27.20 (2015), p. 202202.

[127] Simon Schreck, Annette Pietzsch, Kristjan Kunnus, Brian Kennedy, Wilson Quevedo, Piter S Miedema, Philippe Wernet, and Alexander Föhlisch. "Dynamics of the $\mathrm{OH}$ group and the electronic structure of liquid alcohols." In: Struct. Dyn. 1.5 (2014), p. 054901.

[128] Wayne C Stolte, Ying Lu, James AR Samson, Oliver Hemmers, David L Hansen, Scott B Whitfield, H Wang, Peter Glans, and Dennis W Lindle. "The K-shell Auger decay of atomic oxygen." In: J. Phys. B: At., Mol. Opt. Phys. 30.20 (1997), p. 4489. 
[129] TW Gorczyca, MA Bautista, MF Hasoglu, J García, E Gatuzz, JS Kaastra, TR Kallman, ST Manson, C Mendoza, and AJJ Raassen. "A comprehensive X-ray absorption model for atomic oxygen." In: Astrophys. J. 779.1 (2013), p. 78.

[130] A Menzel, S Benzaid, MO Krause, CD Caldwell, U Hergenhahn, and $\mathrm{M}$ Bissen. "Natural widths in open-shell atoms: The $\mathrm{K}$ absorption spectrum of atomic oxygen." In: Phys. Rev. A 54.2 (1996), R991.

[131] D Petrini and FX De Araujo. "Auger process following 1s-photoionization: O III lines." In: Astron. Astrophys. 282 (1994), pp. 315-317.

[132] B Lohmann and S Fritzsche. "Intensities and angular distribution parameters for the KLL Auger transitions of atomic oxygen." In: J. Phys. B: At., Mol. Opt. Phys. 29.23 (1996), p. 5711.

[133] G Stasińska, N Prantzos, G Meynet, S Simón-Díaz, C Chiappini, M Dessauges-Zavadsky, C Charbonnel, H-G Ludwig, C Mendoza, and N Grevesse. "A panorama of oxygen in the universe." In: EAS Publications Series 54 (2012), pp. 65-186.

[134] Anil K Pradhan, Guo Xin Chen, Franck Delahaye, Sultana N Nahar, and Justin Oelgoetz. "X-ray absorption via $\mathrm{K} \alpha$ resonance complexes in oxygen ions." In: Mon. Not. R. Astron. Soc. 341.4 (2003), pp. 12681271.

[135] A Dalgarno, RJW Henry, and AL Stewart. "The photoionization of atomic oxygen." In: Planet. Space Sci. 12.3 (1964), pp. 235-246.

[136] Robert F Reilman and Steven T Manson. "Photoabsorption cross sections for positive atomic ions with $\mathrm{Z}$ equal to or less than 30." In: Astrophys. J. Supplement Series 40 (1979), pp. 815-880.

[137] DA Verner, DG Yakovlev, IM Band, and MB Trzhaskovskaya. "Subshell photoionization cross sections and ionization energies of atoms and ions from He to Zn." In: At. Data Nucl. Data Tables 55.2 (1993), pp. 233-280.

[138] Brendan M McLaughlin and Kate P Kirby. "Photoabsorption of atomic oxygen in the vicinity of the K-edge." In: J. Phys. B: At., Mol. Opt. Phys. 31.22 (1998), p. 4991.

[139] HP Saha. "Theoretical studies of the K-shell Auger spectrum of atomic oxygen." In: Phys. Rev. A 49.2 (1994), p. 894. 
[140] Brendan M McLaughlin, CP Ballance, KP Bowen, DJ Gardenghi, and Wayne C Stolte. "High precision K-shell photoabsorption cross sections for atomic oxygen: experiment and theory." In: Astrophys. J. Letters 771.1 (2013), p. L8.

[141] Nobuhiro Kosugi, Eiji Shigemasa, and Akira Yagishita. "High-resolution and symmetry-resolved oxygen K-edge spectra of $\mathrm{O}_{2}$." In: Chem. Phys. Lett. 190.5 (1992), pp. 481-488.

[142] RN Dixon and SE Hull. "The photo-ionization of $\pi$-electrons from $\mathrm{O}_{2}$." In: Chem. Phys. Lett. 3.6 (1969), pp. 367-370.

[143] I Hjelte, O Björneholm, V Carravetta, C Angeli, R Cimiraglia, K Wiesner, S Svensson, and MN Piancastelli. "Constant-atomic-final-state filtering of dissociative states in the $\mathrm{O}$ is $\rightarrow \sigma^{*}$ core excitation in $\mathrm{O}_{2}$." In: J. Chem. Phys. 123.6 (2005), p. o64314.

[144] TX Carroll and TD Thomas. "Deexcitation electron spectroscopy of core-excited NO." In: J. Chem. Phys. 97.2 (1992), pp. 894-899.

[145] Pieter Kuiper and Brett I Dunlap. "The $\sigma^{*}$ absorption peak at the oxygen is edge of $\mathrm{O}_{2}$ : Exchange splitting, ultrafast dissociation, and atomiclike Auger spectra." In: J. Chem. Phys. 100.6 (1994), pp. 40874092.

[146] D Lapiano-Smith, K Lee, C-I Ma, KT Wu, and DM Hanson. "Electronic decay of core hole excited states in molecular oxygen." In: J. Chem. Phys. 93.4 (1990), pp. 2169-2175.

[147] SJ Schaphorst, CD Caldwell, MO Krause, and J Jiménez-Mier. "Evidence for atomic features in the decay of resonantly excited molecular oxygen." In: Chem. Phys. Lett. 213.3-4 (1993), pp. 315-320.

[148] MariaNovella Piancastelli, Antti Kivimäki, V Carravetta, I Cacelli, Renzo Cimiraglia, Celestino Angeli, $\mathrm{H}$ Wang, M Coreno, M De Simone, G Turri, et al. "O is $\rightarrow \sigma^{*}$ resonance in $\mathrm{O}_{2}$ : inadequacy of only two exchange-split components." In: Phys. Rev. Lett. 88.24 (2002), p. 243002.

[149] Akira Yagishita, Eiji Shigemasa, and Nobuhiro Kosugi. “Observation of Rydberg-valence mixing in high-resolution symmetry-resolved oxygen K-edge spectra of $\mathrm{O}_{2}$." In: Phys. Rev. Lett. 72.25 (1994), p. 3961.

[150] Hans Ågren, Li Yang, Vincenzo Carravetta, and Lars GM Pettersson. "On the interpretation of the NEXAFS spectrum of molecular oxygen." In: Chem. Phys. Lett. 259.1-2 (1996), pp. 21-27. 
[151] Peter Glans, K Gunnelin, P Skytt, J-H Guo, Nial Wassdahl, J Nordgren, H Ågren, F Kh Gel'Mukhanov, T Warwick, and Eli Rotenberg. "Resonant x-ray emission spectroscopy of molecular oxygen." In: Phys. Rev. Lett. 76.14 (1996), p. 2448.

[152] SL Sorensen, KJ Børve, R Feifel, A de Fanis, and K Ueda. "The O is photoelectron spectrum of molecular oxygen revisited." In: J. Phys. B: At., Mol. Opt. Phys. 41.9 (2008), p. 095101.

[153] M Coreno, M De Simone, KC Prince, R Richter, M Vondráček, L Avaldi, and R Camilloni. "Vibrationally resolved oxygen $\mathrm{K} \rightarrow \Pi$ spectra of $\mathrm{O}_{2}$ and CO." In: Chem. Phys. Lett. 306.5-6 (1999), pp. 269-274.

[154] Raimund Feifel, Yasen Velkov, V Carravetta, C Angeli, R Cimiraglia, P Sałek, Faris Gel'mukhanov, SL Sorensen, MN Piancaśtelli, A De Fanis, et al. "X-ray absorption and resonant Auger spectroscopy of $\mathrm{O}_{2}$ in the vicinity of the $\mathrm{O} \perp \mathrm{s} \rightarrow \sigma^{*}$ resonance: Experiment and theory." In: J. Chem. Phys. 128.6 (2008), p. 064304.

[155] W Wurth, J Stöhr, P Feulner, X Pan, KR Bauchspiess, Y Baba, E Hudel, $\mathrm{G}$ Rocker, and D Menzel. "Bonding, structure, and magnetism of physisorbed and chemisorbed $\mathrm{O}_{2}$ on Pt (111)." In: Phys. Rev. Lett. 65.19 (1990), p. 2426.

[156] JA Sheehy, TJ Gil, CL Winstead, RE Farren, and PW Langhoff. "Correlation of molecular valence-and K-shell photoionization resonances with bond lengths." In: J. Chem. Phys. 91.3 (1989), pp. 1796-1812.

[157] A-P_ Hitchcock and CE Brion. "K-shell excitation spectra of CO, $\mathrm{N}_{2}$ and $\mathrm{O}_{2} . "$ In: J. Electron Spectrosc. Relat. Phenom. 18.1 (1980), pp. 1-21.

[158] RA Rosenberg, PJ Love, PR LaRoe, Victor Rehn, and CC Parks. "Kshell photoexcitation of solid $\mathrm{N}_{2}, \mathrm{CO}, \mathrm{NO}, \mathrm{O}_{2}$, and $\mathrm{N}_{2} \mathrm{O}$." In: Phys. Rev. B 31.5 (1985), p. 2634.

[159] MW Ruckman, Jie Chen, SL Qiu, P Kuiper, Myron Strongin, and BI Dunlap. "Interpreting the near edges of $\mathrm{O}_{2}$ and $\mathrm{O} 2$ 2- in alkali-metal superoxides." In: Physical review letters 67.18 (1991), p. 2533.

[160] M Neeb, J-E Rubensson, M Biermann, and W Eberhardt. "Determination of the exchange splitting of the shape resonance of $\mathrm{O}_{2}$ using the core hole decay spectrum as a "fingerprint."" In: Phys. Rev. Lett. 71.19 (1993), p. 3091. 
[161] Yue Meng, Peter J Eng, S Tse John, Dawn M Shaw, Michael Y Hu, Jinfu Shu, Stephen A Gramsch, Chichang Kao, Russell J Hemley, and Ho-kwang Mao. "Inelastic x-ray scattering of dense solid oxygen: Evidence for intermolecular bonding." In: Proc. Natl. Acad. Sci. (2008).

[162] GR Wight and CE Brion. "K-shell energy loss spectra of $2.5 \mathrm{keV}$ electrons in $\mathrm{CO}_{2}$ and $\mathrm{N}_{2} \mathrm{O}$." In: J. Electron Spectrosc. Relat. Phenom. $3 \cdot 3$ (1974), pp. 191-205.

[163] Nobuhiro Kosugi, Jun-ichi Adachi, Eiji Shigemasa, and Akira Yagishita. "High-resolution and symmetry-resolved $\mathrm{N}$ and $\mathrm{O}$ K-edge absorption spectra of NO." In: J. Chem. Phys. 97.12 (1992), pp. 88428849 .

[164] R Püttner, I Dominguez, TJ Morgan, C Cisneros, RF Fink, E Rotenberg, T Warwick, M Domke, G Kaindl, and AS Schlachter. "Vibrationally resolved $\mathrm{O}$ is core-excitation spectra of $\mathrm{CO}$ and NO." In: Phys. Rev. A 59.5 (1999), p. 3415.

[165] H Brion, C Moser, and M Yamazaki. "Electronic structure of nitric oxide." In: J. Chem. Phys. 30.3 (1959), pp. 673-681.

[166] J Hedman, P-F Heden, C Nordling, and K Siegbahn. "Energy splitting of core electron levels in paramagnetic molecules." In: Phys. Lett. A 29.4 (1969), pp. 178-179.

[167] W E_ Moddeman, Thomas A Carlson, Manfred O Krause, BP Pullen, WE Bull, and GK Schweitzer. "Determination of the K-LL Auger spectra of $\mathrm{N}_{2}, \mathrm{O}_{2}, \mathrm{CO}, \mathrm{NO}, \mathrm{H}_{2} \mathrm{O}$, and $\mathrm{CO}_{2}$." In: J. Chem. Phys. 55.5 (1971), pp. 2317-2336.

[168] Joseph L Dehmer and Dan Dill. "Molecular effects on inner-shell photoabsorption. K-shell spectrum of $\mathrm{N}_{2}$." In: J. Chem. Phys. 65.12 (1976), pp. 5327-5334.

[169] N Padial, G Csanak, BV McKoy, and PW Langhoff. "Photoabsorption in carbon monoxide: Stieltjes-Tchebycheff calculations in the separatedchannel static-exchange approximation." In: J. Chem. Phys. 69.7 (1978), pp. 2992-3004.

[170] Rebecca Lindblad. "High resolution NEXAFS of $\mathrm{N}^{2+}, \mathrm{O}^{2+}$ and $\mathrm{CO}^{+}$ molecular cations." In: BESSY experimental report 17205563 ST (2018).

[171] Rebecca Lindblad. "High resolution NEXAFS of $\mathrm{N}^{2+}, \mathrm{O}^{2+}$ and $\mathrm{CO}^{+}$ molecular cations." In: BESSY experimental report $18106586 S T$ (2018). 
[172] T Gejo, K Okada, and T Ibuki. "Photoabsorption spectrum of ozone in the K-edge region." In: Chem. Phys. Lett. 277.5-6 (1997), pp. 497501.

[173] KC Prince, L Avaldi, M Coreno, R Camilloni, and M De Simone. "Vibrational structure of core to Rydberg state excitations of carbon dioxide and dinitrogen oxide." In: J. Phys. B: At., Mol. Opt. Phys. 32.11 (1999), p. 2551.

[174] Jun-ichi Adachi, Nobuhiro Kosugi, Eiji Shigemasa, and Akira Yagishita. "Renner-Teller effect and Rydberg-valence mixing in the $\mathrm{N}$ and $\mathrm{O}$ K-edge photoabsorption spectra of $\mathrm{N}_{2} \mathrm{O}$." In: J. Chem. Phys. 102.19 (1995), pp. 7369-7376.

[175] Sean R Shieh, Ignace Jarrige, Min Wu, Nozomu Hiraoka, S Tse John, Zhongying Mi, Linada Kaci, Jian-Zhong Jiang, and Yong Q Cai. “Electronic structure of carbon dioxide under pressure and insights into the molecular-to-nonmolecular transition." In: Proc. Natl. Acad. Sci. 110.46 (2013), pp. 18402-18406.

[176] Jacob W Smith and Richard J Saykally. "Soft x-ray absorption spectroscopy of liquids and solutions." In: Chem. Rev. 117.23 (2017), pp. 1390913934.

[177] Anders Nilsson and Lars GM Pettersson. "Perspective on the structure of liquid water." In: Chem. Phys. 389.1-3 (2011), pp. 1-34.

[178] S Myneni, Yi Luo, L Å Näslund, M Cavalleri, L Ojamäe, H Ogasawara, A Pelmenschikov, Ph Wernet, P Väterlein, and C Heske. "Spectroscopic probing of local hydrogen-bonding structures in liquid water." In: J. Phys.: Condens. Matter 14.8 (2002), p. L213.

[179] Jonas A Sellberg, Sarp Kaya, Vegard H Segtnan, Chen Chen, Tolek Tyliszczak, Hirohito Ogasawara, Dennis Nordlund, Lars GM Pettersson, and Anders Nilsson. "Comparison of x-ray absorption spectra between water and ice: New ice data with low pre-edge absorption cross-section." In: The Journal of chemical physics 141.3 (2014), p. 034507.

[180] Michael Odelius, Matteo Cavalleri, Anders Nilsson, and Lars GM Pettersson. "X-ray absorption spectrum of liquid water from molecular dynamics simulations: Asymmetric model." In: Phys. Rev. B 73.2 (2006), p. 024205.

[181] M Cavalleri, H Ogasawara, LGM Pettersson, and A Nilsson. "The interpretation of X-ray absorption spectra of water and ice." In: Chem. Phys. Lett. 364.3-4 (2002), pp. 363-370. 
[182] Dennis Nordlund, Hirohito Ogasawara, Hendrik Bluhm, Osamu Takahashi, Michael Odelius, M Nagasono, Lars GM Pettersson, and Anders Nilsson. "Probing the electron delocalization in liquid water and ice at attosecond time scales." In: Phys. Rev. Lett. 99.21 (2007), p. 217406.

[183] Ph Wernet et al. "The structure of the first coordination shell in liquid water." In: Science 304.5673 (2004), pp. 995-999.

[184] Kathrin M Lange, Kai F Hodeck, Ulrich Schade, and Emad F Aziz. "Nature of the hydrogen bond of water in solvents of different polarities." In: J. Phys. Chem. B 114.50 (2010), pp. 16997-17001.

[185] O Fuchs, M Zharnikov, L Weinhardt, M Blum, M Weigand, Y Zubavichus, M Bär, F Maier, JD Denlinger, and C Heske. "Isotope and temperature effects in liquid water probed by X-ray absorption and resonant X-ray emission spectroscopy." In: Phys. Rev. Lett. 100.2 (2008), p. 027801.

[186] Uwe Bergmann, Dennis Nordlund, Ph Wernet, Michael Odelius, Lars GM Pettersson, and Anders Nilsson. "Isotope effects in liquid water probed by x-ray Raman spectroscopy." In: Phys. Rev. B 76.2 (2007), p. 024202.

[187] AP Hitchcock and DC Mancini. "Bibliography and database of inner shell excitation spectra of gas phase atoms and molecules." In: J. Electron Spectrosc. Relat. Phenom. 67.1 (1994), p. vii.

[188] Yan Zubavichus, Oliver Fuchs, Lothar Weinhardt, Clemens Heske, Eberhard Umbach, Jonathan D Denlinger, and Michael Grunze. "Soft X-ray-induced decomposition of amino acids: an XPS, mass spectrometry, and NEXAFS study." In: Radiat. Res. 161.3 (2004), pp. 346-358.

[189] R Panajotović, S Ptasinska, V Lyamayev, and K Prince. "Low-energy electron damage of DPPC molecules-A NEXAFS study." In: Rad. Applic. 1.1 (2016), pp. 46-50.

[190] RH Wade. "The temperature dependence of radiation damage in organic and biological materials." In: Ultramicroscopy 14.3 (1984), pp. 265270.

[191] Vadim Cherezov, Ken M Riedl, and Martin Caffrey. "Too hot to handle? Synchrotron X-ray damage of lipid membranes and mesophases." In: J. Synchrotron Radiat. 9.6 (2002), pp. 333-341.

[192] Joon Y Lee and Harold C Box. "ESR and ENDOR studies of DL-serine irradiated at 4.2K." In: J. Chem. Phys. 59.5 (1973), pp. 2509-2512. 
[193] Stephen M Adams, Edwin E Budzinski, and Harold C Box. "Primary oxidation and reduction products in x-irradiated aspartic acid." In: J. Chem. Phys. 65.3 (1976), pp. 998-1001.

[194] MA Collins and DH Whiffen. "The electron spin resonance of irradiated glycine at 77K." In: Molecular Physics 10.4 (1966), pp. 317-325.

[195] James W Sinclair and Melvin W Hanna. "Electron paramagnetic resonance study of L-alanine irradiated at low temperatures." In: J. Phys. Chem. 71.1 (1967), pp. 84-88.

[196] BW Castleman and Grace C Moulton. "Electron spin resonance and electron nuclear double resonance studies of DL-Serine. II. Identification of free radicals produced in the range 153-340K." In: J. Chem. Phys. 57.7 (1972), pp. 2762-2768.

[197] Shau-Da Lin. "Electron radiation damage of thin films of glycine, diglycine, and aromatic amino acids." In: Radiat. Res. 59.3 (1974), pp. 521-536.

[198] KS Stenn and GF Bahr. "A study of mass loss and product formation after irradiation of some dry amino acids, peptides, polypeptides and proteins with an electron beam of low current density." In: Journal of Histochemistry \& Cytochemistry 18.8 (1970), pp. 574-580.

[199] Yan Zubavichus, Andrey Shaporenko, Michael Grunze, and Michael Zharnikov. "Innershell absorption spectroscopy of amino acids at all relevant absorption edges." In: J. Phys. Chem. A 109.32 (2005), pp. 6998-7000.

[200] Masahito Tanaka, Kazumichi Nakagawa, Toshiyuki Koketsu, Akane Agui, and Akinari Yokoya. "Oxygen K-edge X-ray absorption near edge structures (XANES) of sublimated films of amino acids." In: J. Synchrotron Radiat. 8.2 (2001), pp. 1009-1011.

[201] DT Clark, J Peeling, and L Colling. "An experimental and theoretical investigation of the core level spectra of a series of amino acids, dipeptides and polypeptides." In: Biochimica et Biophysica Acta (BBA)Protein Structure 453.2 (1976), pp. 533-545.

[202] BM Messer, CD Cappa, JD Smith, KR Wilson, MK Gilles, RC Cohen, and RJ Saykally. " $\mathrm{pH}$ dependence of the electronic structure of glycine." In: J. Phys. Chem. B 109.11 (2005), pp. 5375-5382. 
[203] Kentaro Fujii, Ken Akamatsu, Yasuji Muramatsu, and Akinari Yokoya. "X-ray absorption near edge structure of DNA bases around oxygen and nitrogen K-edge." In: Nucl. Instrum. Methods Phys. Res., Sect. B 199 (2003), pp. 249-254.

[204] Newton T Samuel, Chi-Ying Lee, Lara J Gamble, Daniel A Fischer, and David G Castner. "NEXAFS characterization of DNA components and molecular-orientation of surface-bound DNA oligomers." In: J. Electron Spectrosc. Relat. Phenom. 152.3 (2006), pp. 134-142.

[205] J MacNaughton, A Moewes, and EZ Kurmaev. "Electronic structure of the nucleobases." In: J. Phys. Chem. B 109.16 (2005), pp. 7749-7757.

[206] K Fujii, K Akamatsu, and A Yokoya. "Near-edge X-ray absorption fine structure of DNA nucleobases thin film in the nitrogen and oxygen K-edge region." In: J. Phys. Chem. B 108.23 (2004), pp. 8031-8035.

[207] T Wiell, JE Klepeis, P Bennich, O Björneholm, N Wassdahl, and A Nilsson. "Local aspects of the adsorbate-substrate chemical bond in N/Cu(10o) and O/Cu(10o)." In: Phys. Rev. B 58.3 (1998), p. 1655.

[208] M Grioni, JB Goedkoop, R Schoorl, FMF De Groot, JC Fuggle, F Schäfers, EE Koch, G Rossi, J-M Esteva, and RC Karnatak. "Studies of copper valence states with $\mathrm{Cu} \mathrm{L}_{3}$ x-ray-absorption spectroscopy." In: Phys. Rev. B 39.3 (1989), p. 1541.

[209] Anders Nilsson, Lars GM Pettersson, and Jens Norskov. Chemical bonding at surfaces and interfaces. Elsevier, 2011.

[210] J Stöhr and R Jaeger. "Absorption-edge resonances, core-hole screening, and orientation of chemisorbed molecules: $\mathrm{CO}, \mathrm{NO}$, and $\mathrm{N}_{2}$ on Ni (100)." In: Phys. Rev. B 26.8 (1982), p. 4111.

[211] Helena Tillborg, Anders Nilsson, Nils Martensson, and Jesper N Andersen. "Adsorption-site-dependent x-ray-absorption spectroscopy: $\mathrm{CO} / \mathrm{H}$, $\mathrm{H}_{2} / \mathrm{Ni}$ (100)." In: Phys. Rev. B 47.3 (1993), p. 1699.

[212] Verena Pfeifer, Travis E Jones, Juan J Velasco Vélez, Cyriac Massué, Rosa Arrigo, Detre Teschner, Frank Girgsdies, Michael Scherzer, Mark T Greiner, and Jasmin Allan. "The electronic structure of iridium and its oxides." In: Surf. Interface Anal. 48.5 (2016), pp. 261-273.

[213] Marc F Tesch, Shannon A Bonke, Travis E Jones, Maryam N Shaker, Jie Xiao, Katarzyna Skorupska, Rik Mom, Jens Melder, Philipp Kurz, and Axel Knop-Gericke. "Evolution of oxygen-metal electron transfer and metal electronic states during manganese oxide catalyzed water 
oxidation revealed with in situ soft X-ray spectroscopy." In: Angew. Chem. 131.11 (2019), pp. 3464-3470.

[214] Rodney J. Bartlett and Monika Musial. "Coupled-cluster theory in quantum chemistry." In: Rev. Mod. Phys. 79 (2007), pp. 291-352.

[215] Trygve Helgaker, Sonia Coriani, Poul Jørgensen, Kasper Kristensen, Jeppe Olsen, and Kenneth Ruud. "Recent Advances in Wave FunctionBased Methods of Molecular-Property Calculations." In: Chem. Rev. 112 (2012), pp. 543-631.

[216] O. Christiansen, P. Jørgensen, and C. Hättig. In: Int. J. Quantum Chem. 98 (1998), p. 1.

[217] O. Christiansen. "Coupled Cluster Theory with Emphasis on Selected New Developments." In: Theor. Chem. Accounts 116 (2006), p. 106.

[218] S. Coriani and H. Koch. "Communication: X-ray Absorption Spectra and Core-Ionization Potentials within a Core-Valence Separated Coupled Cluster Framework." In: J. Chem. Phys. 143 (2015), p. 181103.

[219] G Remmers, M Domke, A Puschmann, T Mandel, C Xue, G Kaindl, E Hudson, and DA Shirley. "High-resolution K-shell photoabsorption in formaldehyde." In: Phys. Rev. A 46.7 (1992), p. 3935.

[220] T. Helgaker, P. Jørgensen, and J. Olsen. Molecular Electronic Structure Theory. Wiley, 2004.

[221] Trygve Helgaker, Poul Jorgensen, and Jeppe Olsen. Molecular electronicstructure theory. John Wiley \& Sons, 2014.

[222] Henrik Koch and Poul Jørgensen. "Coupled Cluster Response Functions." In: J. Chem. Phys. 93 (1990), pp. 3333-3344.

[223] Ernest R. Davidson. "The iterative calculation of a few of the lowest eigenvalues and corresponding eigenvectors of large real-symmetric matrices." In: J. Comput. Phys. 17.1 (1975), pp. 87 -94. ISSN: 0021-9991.

[224] S. Coriani, O. Christiansen, T. Fransson, and P. Norman. "CoupledCluster Response Theory for Near-Edge X-Ray-Absorption Fine Structure of Atoms and Molecules." In: Phys. Rev. A 85 (2012), p. 022507.

[225] Sonia Coriani, Thomas Fransson, Ove Christiansen, and Patrick Norman. "Asymmetric-Lanczos-chain-driven implementation of electronic resonance convergent coupled-cluster linear response theory." In: J. Chem. Theory Comput. 8.5 (2012), pp. 1616-1628. 
[226] O. Christiansen, A. Halkier, H. Koch, P. Jørgensen, and T. Helgaker. "Integral-direct Coupled Cluster Calculations of Frequency-Dependent Polarizabilities Transition Probabilities and Excited-State Properties." In: J. Chem. Phys. 108 (1998), p. 2801.

[227] L. S. Cederbaum, W. Domcke, and J. Schirmer. "Many-body theory of core holes." In: Phys. Rev. A: At. Mol. Opt. Phys. 22 (1980), pp. 206222.

[228] Fabrizio Santoro, Roberto Improta, Alessandro Lami, Julien Bloino, and Vincenzo Barone. "Effective method to compute Franck-Condon integrals for optical spectra of large molecules in solution." In: $J$. Chem. Phys. 126.8 (2007), p. 084509.

[229] Francisco José Avila Ferrer, Javier Cerezo, Juan Soto, Roberto Improta, and Fabrizio Santoro. "First-principle computation of absorption and fluorescence spectra in solution accounting for vibronic structure, temperature effects and solvent inhomogenous broadening." In: Computational and Theoretical Chemistry 1040 (2014), pp. 328-337.

[23o] Filip Pawłowski, Poul Jørgensen, Jeppe Olsen, Flemming Hegelund, Trygve Helgaker, Jürgen Gauss, Keld L Bak, and John F Stanton. "Molecular equilibrium structures from experimental rotational constants and calculated vibration-rotation interaction constants." In: J. Chem. Phys. 116.15 (2002), pp. 6482-6496.

[231] Munetaka Nakata, Kunio Kohata, Tsutomu Fukuyama, Kozo Kuchitsu, and CJ Wilkins. "Molecular structure of carbonyl fluoride as studied by gas electron diffraction and microwave data." In: Journal of Molecular Structure 68 (1980), pp. 271-280.

[232] George Hsing Kwei and RF Curl Jr. "Microwave spectrum of O18 formic acid and structure of formic acid." In: J. Chem. Phys. 32.5 (1960), pp. 1592-1594.

[233] Ronald F Miller and RF Curl Jr. "Microwave Spectrum of O18 Formyl Fluoride and the Structure of Formyl Fluoride." In: J. Chem. Phys. 34.5 (1961), pp. 1847-1848.

[234] K. Aidas et al. "The Dalton Quantum Chemistry Program System." In: WIREs Comput. Mol. Sci. 4 (2014), p. 269.

[235] Thom H Dunning and P Jeffrey Hay. "Gaussian basis sets for molecular calculations." In: Methods of electronic structure theory. Springer, 1977, pp. 1-27. 
[236] K. Kaufmann, W. Baumeister, and M. Jungen. In: J. Phys. B: At., Mol. Opt. Phys. 22 (1989), p. 2223.

[237] J. F. Stanton, J. Gauss, L. Cheng, M. E. Harding, D. A. Matthews, and P. G. Szalay. CFOUR, Coupled-Cluster techniques for Computational Chemistry, a quantum-chemical program package. With contributions from A.A. Auer, R.J. Bartlett, U. Benedikt, C. Berger, D.E. Bernholdt, Y.J. Bomble, O. Christiansen, F. Engel, R. Faber, M. Heckert, O. Heun, M. Hilgenberg, C. Huber, T.-C. Jagau, D. Jonsson, J. Jusélius, T. Kirsch, K. Klein, W.J. Lauderdale, F. Lipparini, T. Metzroth, L.A. Mück, D.P. O'Neill, D.R. Price, E. Prochnow, C. Puzzarini, K. Ruud, F. Schiffmann, W. Schwalbach, C. Simmons, S. Stopkowicz, A. Tajti, J. Vázquez, F. Wang, J.D. Watts and the integral packages MOLECULE (J. Almlöf and P.R. Taylor), PROPS (P.R. Taylor), ABACUS (T. Helgaker, H.J. Aa. Jensen, P. Jørgensen, and J. Olsen), and ECP routines by A. V. Mitin and C. van Wüllen. For the current version, see http:/ / www.cfour.de.

[238] Jürgen Gauss, John F. Stanton, and Rodney J. Bartlett. "Coupled-cluster open-shell analytic gradients: Implementation of the direct product decomposition approach in energy gradient calculations." In: J. Chem. Phys. 95.4 (1991), pp. 2623-2638.

[239] Henrik Koch, Hans Jørgen Aa. Jensen, Poul Jørgensen, Trygve Helgaker, Gustavo E. Scuseria, and Henry F. Schaefer. “Coupled cluster energy derivatives. Analytic Hessian for the closed-shell coupled cluster singles and doubles wave function: Theory and applications." In: J. Chem. Phys. 92.8 (1990), pp. 4924-4940.

[240] Jürgen Gauss and John F. Stanton. "Analytic CCSD(T) second derivatives." In: Chem. Phys. Lett. 276.1 (1997), pp. 70 -77. IssN: 00o9-2614.

[241] John F. Stanton and Jürgen Gauss. "Analytic second derivatives in high-order many-body perturbation and coupled-cluster theories: Computational considerations and applications." In: Int. Reviews Phys. Chem. 19.1 (2000), pp. 61-95.

[242] John F. Stanton. "Many-body methods for excited state potential energy surfaces. I. General theory of energy gradients for the equationof-motion coupled-cluster method." In: J. Chem. Phys. 99.11 (1993), pp. $8840-8847$.

[243] John F. Stanton and Jürgen Gauss. "Analytic energy derivatives for the equation-of-motion coupled-cluster method: Algebraic expressions, implementation and application to the Si state of HFCO." In: Theoret. Chim. Acta 91.5 (1995), pp. 267-289. ISSN: 1432-2234. 
[244] Francisco Jose Avila Ferrer and Fabrizio Santoro. "Comparison of vertical and adiabatic harmonic approaches for the calculation of the vibrational structure of electronic spectra." In: Phys. Chem. Chem. Phys. 14.39 (2012), pp. 13549-13563.

[245] Fabrizio Santoro and Javier Cerezo. FCclasses v. 3.o. Available upon request.

[246] Thom H Dunning Jr. "Gaussian basis sets for use in correlated molecular calculations. I. The atoms boron through neon and hydrogen." In: J. Chem. Phys. 90.2 (1989), pp. 1007-1023.

[247] Nitai Sylvetsky, Manoj K Kesharwani, and Jan ML Martin. “The augcc-pVnZ-F12 basis set family: Correlation consistent basis sets for explicitly correlated benchmark calculations on anions and noncovalent complexes." In: J. Chem. Phys. 147.13 (2017), p. 134106.

[248] SF Machado, GG Camiletti, A Canal Neto, FE Jorge, and Raquel S Jorge. "Gaussian basis set of triple zeta valence quality for the atoms from K to Kr: Application in DFT and CCSD (T) calculations of molecular properties." In: Mol. Phys. 107.16 (2009), pp. 1713-1727.

[249] Marvin Douglas and Norman M Kroll. "Quantum electrodynamical corrections to the fine structure of helium." In: Annals of Physics 82.1 (1974), pp. 89-155.

[250] Bernd A Hess. "Relativistic electronic-structure calculations employing a two-component no-pair formalism with external-field projection operators." In: Phy. Rev. A 33.6 (1986), p. 3742.

[251] Kenneth G. Dyall. "Interfacing relativistic and nonrelativistic methods. I. Normalized elimination of the small component in the modified Dirac equation." In: J. Chem. Phys. 106.23 (1997), pp. 9618-9626.

[252] Wenjian Liu and Daoling Peng. "Exact two-component Hamiltonians revisited." In: J. Chem. Phys. 131.3 (2009), p. 031104.

[253] Motomichi Tashiro, Masahiro Ehara, Hironobu Fukuzawa, Kiyoshi Ueda, Christian Buth, Nikolai V. Kryzhevoi, and Lorenz S. Cederbaum. "Molecular double core hole electron spectroscopy for chemical analysis." In: J. Chem. Phys. 132.18 (2010), p. 184302. ISSN: 00219606.

[254] Nicholas A Besley, Michael J G Peach, and David J Tozer. “Timedependent density functional theory calculations of near-edge $\mathrm{X}$-ray absorption fine structure with short-range corrected functionals." In: Phys. Chem. Chem. Phys. 11.44 (2009), pp. 10350-10358. 
[255] Prakash Verma, Wallace D Derricotte, and Francesco A Evangelista. "Predicting Near Edge X-ray Absorption Spectra with the Spin-Free Exact-Two-Component Hamiltonian and Orthogonality Constrained Density Functional Theory." In: J. Chem. Theory Comput. 12.1 (2016), pp. $144-156$.

[256] Rolf H. Myhre, Thomas J. A. Wolf, Lan Cheng, Saikat Nandi, Sonia Coriani, Markus Gühr, and Henrik Koch. "A theoretical and experimental benchmark study of core-excited states in nitrogen." In: J. Chem. Phys. 148 (2018), p. 064106.

[257] Katherine J. Oosterbaan, Alec F. White, and Martin Head-Gordon. "Non-orthogonal configuration interaction with single substitutions for the calculation of core-excited states." In: J. Chem. Phys. 149.4 (2018), p. 044116.

[258] Katherine J. Oosterbaan, Alec F. White, and Martin Head-Gordon. "Erratum: "Non-orthogonal configuration interaction with single substitutions for the calculation of core-excited states" [J. Chem. Phys. 149, 044116 (2018)]." In: J. Chem. Phys. 149.13 (2018), p. 139901.

[259] O. Christiansen, H. Koch, and P. Jørgensen. "The second-order approximate coupled cluster singles and doubles model CC2." In: Chem. Phys. Lett. 243 (1995), p. 409.

[26o] Henrik Koch, Ove Christiansen, Poul Jørgensen, and Jeppe Olsen. "Excitation energies of $\mathrm{BH}, \mathrm{CH} 2$ and $\mathrm{Ne}$ in full configuration interaction and the hierarchy CCS, CC2, CCSD and $\mathrm{CC}_{3}$ of coupled cluster models." In: Chem. Phys. Lett. 244.1-2 (1995), pp. 75-82.

[261] Kevin C Prince, Robert Richter, Monica de Simone, Michele Alagia, and Marcello Coreno. "Near edge X-ray absorption spectra of some small polyatomic molecules." In: J. Phys. Chem. A 107.12 (2003), pp. 19551963.

[262] MB Robin, I Ishii, R McLaren, and AP Hitchcock. "Fluorination effects on the inner-shell spectra of unsaturated molecules." In: J. Electron Spectrosc. 47 (1988), pp. 53-92.

[263] A B Trofimov, E V Gromov, H Köppel, J Schirmer, K C Prince, R Richter, $\mathrm{M}$ De Simone, and M Coreno. "A theoretical study of the ${ }^{1} \mathrm{~B}_{1}$ (O1s $\rightarrow \pi^{*}$ ) and ${ }^{1} \mathrm{~A}_{1}$ (O $\left.\mathrm{O}_{1 \mathrm{~s}} \rightarrow 3 \mathrm{~s}\right)$ excited states of formaldehyde." In: J. Phys. B: At. Mol. Opt. Phys. 36.18 (2003), p. 3805. 
[264] I Ishii and AP Hitchcock. "A quantitative experimental study of the core excited electronic states of formamide, formic acid, and formyl fluoride." In: J. Chem. Phys. 87.2 (1987), pp. 830-839.

[265] Peter H Cribb and Jürgen Brickmann. "Time Delayed Two Photon Processes: II. Duschinsky Mixing Effects." In: Berichte der Bunsengesellschaft für physikalische Chemie 90.2 (1986), pp. 168-175.

[266] A. B. Trofimov, T. E. Moskovskaya, E. V. Gromov, H. Köppel, and J. Schirmer. "Theoretical study of K-shell excitations in formaldehyde." In: Phys. Rev. A 64 (2 2001), p. 022504.

[267] A. B. Trofimov, E. V. Gromov, T. E. Moskovskaya, and J. Schirmer. "Theoretical evidence for a bound doubly-excited ${ }^{1} \mathrm{~B}_{2}$ (C1s, $\mathrm{n} \rightarrow \pi^{* 2}$ ) state in $\mathrm{H}_{2} \mathrm{CO}$ below the C1s ionization threshold." In: J. Chem. Phys. 113.16 (2000), pp. 6716-6723.

[268] David Picconi, Alessandro Lami, and Fabrizio Santoro. "Hierarchical transformation of Hamiltonians with linear and quadratic couplings for nonadiabatic quantum dynamics: Application to the $\pi \pi^{*} / \mathrm{n} \pi^{*}$ internal conversion in thymine." In: J. Chem. Phys. 136.24 (2012), p. 244104.

[269] Javier Cerezo, Yanli Liu, Na Lin, Xian Zhao, Roberto Improta, and Fabrizio Santoro. "Mixed Quantum/Classical Method for Nonadiabatic Quantum Dynamics in Explicit Solvent Models: The $\pi \pi^{*} / \mathrm{n} \pi^{*}$ Decay of Thymine in Water as a Test Case." In: J. Chem. Theory Comput. 14.2 (2018), pp. 820-832.

[270] "Multistate coupled quantum dynamics of photoexcited cytosine in gas-phase: Nonadiabatic absorption spectrum and ultrafast internal conversions." In: Chem. Phys. 515 (2018). Special issue on "Ultrafast Photoinduced Processes in Polyatomic Molecules: Electronic Structure, Dynamics and Spectroscopy", dedicated to Wolfgang Domcke on the occasion of his 7oth birthday, pp. $452-463$. ISSN: 0301-0104.

[271] M. Pedio, Z. Y. Wu, M. Benfatto, A. Mascaraque, E. Michel, C. Ottaviani, C. Crotti, M. Peloi, M. Zacchigna, and C. Comicioli. “NEXAFS experiment and multiple scattering calculations on $\mathrm{KO}_{2}$ : Effects on the $\pi$ resonance in the solid phase." In: Phys. Rev. B 66.14 (Oct. 2002), p. 144109 .

[272] J.-S. Kang, D. H. Kim, J. H. Hwang, J. Baik, H. J. Shin, Minjae Kim, Y. H. Jeong, and B. I. Min. "Soft x-ray absorption and photoemission spectroscopy study of superoxide $\mathrm{KO}_{2}$." In: Phys. Rev. B 82.19 (Nov. 2010), p. 193102. 
[273] Ruimin Qiao, Yi-De Chuang, Shishen Yan, and Wanli Yang. "Soft Xray irradiation effects of $\mathrm{Li}_{2} \mathrm{O}_{2}, \mathrm{Li}_{2} \mathrm{CO}_{3}$ and $\mathrm{Li}_{2} \mathrm{O}$ revealed by absorption spectroscopy." en. In: PLOS ONE 7.11 (Nov. 2012), e49182. ISSN: 1932-6203.

[274] Shang-Di Mo and W. Y. Ching. "X-ray absorption near-edge structure in alpha-quartz and stishovite: $\mathrm{Ab}$ initio calculation with core-hole interaction." In: Appl. phys. Lett. 78.24 (June 2001), pp. 3809-3811. IssN: 0003-6951.

[275] H. M. Wang and G. S. Henderson. "Investigation of coordination number in silicate and germanate glasses using $\mathrm{O}$ K-edge $\mathrm{X}$-ray absorption spectroscopy." In: Chem. Geol. 7th Silicate Melt Workshop 213.1-3 (Dec. 2004), pp. 17-30. ISSN: 0009-2541.

[276] John A. McLeod, Nikolai A. Skorikov, Larisa D. Finkelstein, Ernst Z. Kurmaev, and Alexander Moewes. "Chemical Bonding and Hybridization in 5p Binary Oxide." In: J. Phys. Chem. C 116.45 (Nov. 2012), pp. 24248-24254. ISSN: 1932-7447.

[277] Ziyu Wu, Friedrich Seifert, Brent Poe, and Thomas Sharp. "Multiplescattering calculations for $\mathrm{SiO}_{2}$ polymorphs: a comparison to ELNES and XANES spectra." en. In: J. Phys.: Condens. Matter 8.19 (1996), p. 3323. ISSN: 0953-8984.

[278] Z. Y. Wu, F. Jollet, and F. Seifert. "Electronic structure analysis of $\alpha-$ $\mathrm{SiO}_{2}$ via x-ray absorption near-edge structure at the $\mathrm{Si} \mathrm{K}, \mathrm{L}_{2,3}$ and $\mathrm{O}$ K edges." en. In: J. Phys.: Condens. Matter 10.36 (1998), p. 8083. Issn: 0953-8984.

[279] Mathieu Taillefumier, Delphine Cabaret, Anne-Marie Flank, and Francesco Mauri. "X-ray absorption near-edge structure calculations with the pseudopotentials: Application to the K edge in diamond and $\alpha$-quartz." en. In: Phys. Rev. B 66.19 (Nov. 2002). IssN: 0163-1829, 1095-3795.

[280] Delphine Cabaret, Francesco Mauri, and Grant S. Henderson. “Oxygen K-edge XANES of germanates investigated using first-principles calculations." In: Phys. Rev. B 75.18 (May 2007), p. 184205.

[281] F. Jollet and C. Noguera. "Core Hole Effect on the XAS Si K Edge Shape in $\alpha$-Quartz." en. In: Phys. Status Solidi B 179.2 (Oct. 1993), pp. 473-488. ISSN: 03701972, 15213951.

[282] Gérald Lelong, Guillaume Radtke, Laurent Cormier, Hanane Bricha, Jean-Pascal Rueff, James M. Ablett, Delphine Cabaret, Frédéric Gélébart, and Abhay Shukla. "Detecting Non-bridging Oxygens: Non-Resonant 
Inelastic X-ray Scattering in Crystalline Lithium Borates." In: Inorg. Chem. 53.20 (Oct. 2014), pp. 10903-10908. ISSN: 0020-1669.

[283] Gérald Lelong, Laurent Cormier, Louis Hennet, Florent Michel, JeanPascal Rueff, James M. Ablett, and Giulio Monaco. "Lithium borate crystals and glasses: How similar are they? A non-resonant inelastic X-ray scattering study around the B and O K-edges." In: J. Non-Cryst. Solids 472 (Sept. 2017), pp. 1-8. IssN: 0022-3093.

[284] Sung Keun Lee, Peter J. Eng, Ho-kwang Mao, Yue Meng, Matthew Newville, Michael Y. Hu, and Jinfu Shu. "Probing of bonding changes in $\mathrm{B}_{2} \mathrm{O}_{3}$ glasses at high pressure with inelastic X-ray scattering." In: Nat. Mater. 4.11 (Nov. 2005), pp. 851-854. ISSN: 1476-1122.

[285] Osman Karslıoğlu, Lena Trotochaud, Ioannis Zegkinoglou, and Hendrik Bluhm. "X-Ray spectroscopic characterization of $\mathrm{BaO}, \mathrm{Ba}(\mathrm{OH})_{2}$, $\mathrm{BaCO}_{3}$, and $\mathrm{Ba}\left(\mathrm{NO}_{3}\right)_{2}$." In: J. Electron Spectrosc. Relat. Phenom. 225 (2018), pp. 55-61.

[286] Ross T DeVol, Rebecca A Metzler, Lee Kabalah-Amitai, Boaz Pokroy, Yael Politi, Assaf Gal, Lia Addadi, Steve Weiner, Alejandro FernandezMartinez, and Raffaella Demichelis. "Oxygen spectroscopy and polarizationdependent imaging contrast (PIC)-mapping of calcium carbonate minerals and biominerals." In: J. Phys. Chem. B 118.28 (2014), pp. 84498457 .

[287] Laura Espinal, Martin L Green, Daniel A Fischer, Dean M DeLongchamp, Cherno Jaye, Jarod C Horn, Miles A Sakwa-Novak, Watcharop Chaikittisilp, Nicholas A Brunelli, and Christopher W Jones. "Interrogating the carbon and oxygen K-Edge NEXAFS of a $\mathrm{CO}_{2}$-dosed hyperbranched aminosilica." In: J. Phys. Chem. Lett. 6.1 (2014), pp. 148-152.

[288] G. J. Baker, G. N. Greaves, M. Surman, and M. Oversluizen. "An oxygen XAFS study of sodium disilicate glass surfaces." In: Nucl. Instrum. Methods Phys. Res., Sect. B 97.I (May 1995), pp. 375-382. IssN: 0168-583X.

[289] Osman Karslioğlu, Lena Trotochaud, Ioannis Zegkinoglou, and Hendrik Bluhm. "X-Ray Spectroscopic Characterization of $\mathrm{BaO}, \mathrm{Ba}(\mathrm{OH})_{2}$, $\mathrm{BaCO}_{3}$, and $\mathrm{Ba}\left(\mathrm{NO}_{3}\right)_{2}$." In: J. Electron Spectrosc. Relat. Phenom. 225 (May 2018), pp. 55-61. ISSN: 0368-2048.

[290] R.L. Rudnick and S. Gao. "Composition of the Continental Crust." en. In: Treatise on Geochemistry. Elsevier, 2014, pp. 1-51. ISBN: 978-0o8-098300-4. 
[291] S Köstlmeier and C Elsässer. "Ab initio calculation of near-edge structures in electron-energy-loss spectra for metal-oxide crystals." In: Phys. Rev. B 60.20 (1999), p. 14025 .

[292] K Kaneko, T Gemming, I Tanaka, and H Müllejans. "Analytical investigation of random grain boundaries of $\mathrm{Zr}$-doped sintered $\alpha-\mathrm{Al}_{2} \mathrm{O}_{3}$ by transmission electron microscopy and scanning transmission electron microscopy." In: Philos. Mag. A 77.5 (1998), pp. 1255-1272.

[293] Grant S Henderson, Daniel R Neuville, and Laurent Cormier. “An O K-edge XANES study of glasses and crystals in the $\mathrm{CaO}-\mathrm{Al}_{2} \mathrm{O}_{3}-\mathrm{SiO}_{2}$ (CAS) system." In: Chem. Geol. 259.1-2 (2009), pp. 54-62.

[294] Grant S Henderson, Daniel R Neuville, and Laurent Cormier. "An O K-edge XANES study of calcium aluminates." In: Can. J. Chem. 85.10 (Oct. 2007), pp. 801-805. IssN: 0008-4042.

[295] Ping-Hung Hsieh, Yang-Ming Lu, Weng-Sing Hwang, Wei-Luen Jang, Chung-Li Dong, and Ting-Shan Chan. "X-ray absorption spectroscopy study of thermally annealed $\mathrm{Cu}-\mathrm{Al}-\mathrm{O}$ thin films." In: Mater. Chem. Phys. 144.3 (2014), pp. 547-552.

[296] Zhiqiang Wang, Chunlei Li, Lijia Liu, and Tsun-Kong Sham. "Probing defect emissions in bulk, micro-and nano-sized $\alpha-\mathrm{Al}_{2} \mathrm{O}_{3}$ via $X$-ray excited optical luminescence." In: J. Chem. Phys. 138.8 (2013), p. 084706.

[297] A. Marcelli et al. "Local Structure in $\mathrm{SiO}_{2}$ glasses by oxygen $\mathrm{K}$ edge XANES." en. In: Le Journal de Physique Colloques 46.C8 (Dec. 1985), pp. C8-107-C8-112. IssN: 0449-1947.

[298] I. Davoli, E. Paris, S. Stizza, M. Benfatto, M. Fanfoni, A. Gargano, A. Bianconi, and F. Seifert. "Structure of densified vitreous silica: Silicon and oxygen XANES spectra and multiple scattering calculations." en. In: Phys. Chem. Miner. 19.3 (Sept. 1992), pp. 171-175. ISSN: 0342-1791, 1432-2021.

[299] Yasunori Tabira. "Local structure around oxygen atoms in $\mathrm{CaMgSi}_{2} \mathrm{O}_{6}$ glass by O K-edge EXELFS." In: Mater. Sci. Eng.:B 41.1 (Oct. 1996), pp. 63-66. ISSN: 0921-5107.

[300] G. S. Henderson, Xiaoyang Liu, and M. E. Fleet. “Titanium coordination in silicate glasses investigated using $\mathrm{O}$ K-edge X-ray absorption spectroscopy." In: Mineral. Mag. 67.4 (Aug. 2003), pp. 597-607. ISSN: 14718022, 0026461X. 
[301] Grant S. Henderson, Daniel R. Neuville, and Laurent Cormier. "An O K-edge XANES study of glasses and crystals in the $\mathrm{CaO}-\mathrm{Al}_{2} \mathrm{O}_{3}-\mathrm{SiO}_{2}$ (CAS) system." In: Chem. Geol. Experimental techniques for the study of hydrothermal fluids and silicate melts 259.1-2 (Feb. 2009), pp. 5462. ISSN: 0009-2541.

[302] Nan Jiang. "On detection of non-bridging oxygen in glasses by electronenergy-loss spectroscopy." In: Solid State Commun. 122.1-2 (Apr. 2002), pp. 7-10. ISSN: 0038-1098.

[303] G. Lelong. "Evidence of fivefold-coordinated Ge atoms in amorphous GeO." In: Phys. Rev. B 85.13 (2012).

[304] John A. McLeod, Jia Zhao, Linju Yang, Yi Liu, and Lijia Liu. "Structural evolution of reduced $\mathrm{GeO}_{x}$ nanoparticles." en. In: Phys. Chem. Chem. Phys. 19.4 (Jan. 2017), pp. 3182-3191. IssN: 1463-9084.

[305] JA McLeod, RG Wilks, NA Skorikov, LD Finkelstein, M Abu-Samak, EZ Kurmaev, and A Moewes. "Band gaps and electronic structure of alkaline-earth and post-transition-metal oxides." In: Phys. Rev. B 81.24 (2010), p. 245123.

[306] JH Guo, L Vayssieres, Clas Persson, R Ahuja, Börje Johansson, and J Nordgren. "Polarization-dependent soft-x-ray absorption of highly oriented ZnO microrod arrays." In: J. Phys.: Condens. Matter 14.28 (2002), p. 6969.

[307] Ashok Kumar Yadav, Sk Maidul Haque, Shilpa Tripathi, Dinesh Shukla, Md A Ahmed, DM Phase, S Bandyopadhyay, SN Jha, and D Bhattacharyya. "Investigation of Fe doped $\mathrm{ZnO}$ thin films by X-ray absorption spectroscopy." In: RSC Advances 6.78 (2016), pp. 74982-7499o.

[308] Yen-Fa Liao, Tzu-Wen Huang, Ming-Zhe Lin, Kuan-Li Yu, Hua-Shu Hsu, Tsung-Hsun Lee, Chih-Hao Lee, and Jung-Chun Andrew Huang. "X-ray study of the structure and magnetic property of the Co-doped $\mathrm{ZnO}$ nanoparticles prepared by thermal hydrolysis." In: J. Magn. Magn. Mater. 310.2 (2007), e818-e820.

[309] S Krishnamurthy, C McGuinness, LS Dorneles, M Venkatesan, JMD Coey, JG Lunney, $\mathrm{CH}$ Patterson, KE Smith, T Learmonth, and P-A Glans. "Soft-x-ray spectroscopic investigation of ferromagnetic Codoped ZnO." In: J. Appl. Phys. 99.8 (2006), p. o8Mi11.

[310] Douglas M Detert, Kyle B Tom, Corsin Battaglia, Jonathan D Denlinger, Sunnie HN Lim, Ali Javey, André Anders, Oscar D Dubon, Kin M Yu, and Wladek Walukiewicz. "Fermi level stabilization and 
band edge energies in $\mathrm{Cd}_{x} \mathrm{Zn}_{1-x} \mathrm{O}$ alloys." In: J. Appl. Phys. 115.23 (2014), p. 233708.

[311] Jayapradhi Rajendran, Stefano Gialanella, and Pranesh B Aswath. "XANES analysis of dried and calcined bones." In: Materials Science and Engineering: C 33.7 (2013), pp. 3968-3979.

[312] Manu Hegde, Ian D Hosein, and Pavle V Radovanovic. "Molecular Origin of Valence Band Anisotropy in Single $\beta-\mathrm{Ga}_{2} \mathrm{O}_{3}$ Nanowires Investigated by Polarized X-Ray Absorption Imaging." In: J. Phys. Chem. C 119.30 (2015), pp. 17450-17457.

[313] Caterina Cocchi, Hannes Zschiesche, Dmitrii Nabok, Anna Mogilatenko, Martin Albrecht, Zbigniew Galazka, Holm Kirmse, Claudia Draxl, and Christoph T Koch. "Atomic signatures of local environment from core-level spectroscopy in $\beta-\mathrm{Ga}_{2} \mathrm{O}_{3}$." In: Phys. Rev. B 94.7 (2016), p. 075147.

[314] IN Demchenko, M Chernyshova, T Tyliszczak, JD Denlinger, KM Yu, DT Speaks, Oliver Hemmers, W Walukiewicz, G Derkachov, and K Lawniczak-Jablonska. "Electronic structure of $\mathrm{CdO}$ studied by soft X-ray spectroscopy." In: J. Electron Spectrosc. Relat. Phenom. 184.3-6 (2011), pp. 249-253.

[315] T De Boer, MF Bekheet, A Gurlo, R Riedel, and A Moewes. “Band gap and electronic structure of cubic, rhombohedral, and orthorhombic $\mathrm{In}_{2} \mathrm{O}_{3}$ polymorphs: Experiment and theory." In: Phys. Rev. B 93.15 (2016), p. 155205.

[316] Nan Jiang and John CH Spence. "Modeling core-hole effects in electron energy-loss spectroscopy of $\mathrm{TeO}_{2}$." In: Phys. Rev. B 70.1 (2004), p. 014112.

[317] MS Moreno, RF Egerton, and PA Midgley. "Differentiation of tin oxides using electron energy-loss spectroscopy." In: Phys. Rev. B 69.23 (2004), p. 233304.

[318] SI Kurganskii, MD Manyakin, OI Dubrovskii, OA Chuvenkova, S Yu Turishchev, and EP Domashevskaya. "Theoretical and experimental study of the electronic structure of tin dioxide." In: Phys. Solid State 56.9 (2014), pp. 1748-1753.

[319] Coleman X Kronawitter, Mukes Kapilashrami, Jonathan R Bakke, Stacey F Bent, Cheng-Hao Chuang, Way-Faung Pong, Jinghua Guo, Lionel Vayssieres, and Samuel S Mao. " $\mathrm{TiO}_{2}-\mathrm{SnO}_{2}$ : F interfacial electronic structure investigated by soft x-ray absorption spectroscopy." In: Phys. Rev. B 85.12 (2012), p. 125109. 
[320] A Qamar, K LeBlanc, O Semeniuk, A Reznik, J Lin, Y Pan, and A Moewes. "X-ray spectroscopic study of amorphous and polycrystalline $\mathrm{PbO}$ films, $\alpha-\mathrm{PbO}$, and $\beta-\mathrm{PbO}$ for direct conversion imaging." In: Sci. Rep. 7.1 (2017), p. 13159.

[321] P T Moseley and A Cooper. "Progress towards an advanced lead-acid battery for use in electric vehicles." en. In: J. Power Sources (1999), p. 7.

[322] FMF de Groot, JC Fuggle, and JM van Ruitenbeek. "Oxygen 1s x-ray absorption of $\mathrm{BaPb}_{1-\chi} \mathrm{Bi}_{x} \mathrm{O}_{3}$." In: Phys. Rev. B 44.10 (1991), p. 5280.

[323] V. R. Mastelaro, P. P. Neves, S. R. de Lazaro, E. Longo, A. Michalowicz, and J. A. Eiras. "Electronic structure of $\mathrm{Pb}_{1-\chi} \mathrm{La}_{x} \mathrm{TiO}_{3}$ ferroelectric materials from $\mathrm{Ti} 2 \mathrm{p}$ and $\mathrm{O}$ is soft x-ray absorption spectroscopy." In: J. Appl. Phys. 99.4 (Feb. 2006), p. 044104. ISSN: 0021-8979.

[324] V. R. Mastelaro, P. P. Neves, A. Michalowicz, and J. A. Eiras. "Electronic structure of $\mathrm{Pb}_{1-\chi} \mathrm{Ba}_{x} \mathrm{Zr}_{0.65} \mathrm{Ti}_{0.35} \mathrm{O}_{3}$ ferroelectric compounds probed by soft x-ray absorption spectroscopy." en. In: J. Phys.: Condens. Matter 19.22 (2007), p. 226212. ISSN: 0953-8984.

[325] E. Eberg, A. T. J. van Helvoort, R. Takahashi, M. Gass, B. Mendis, A. Bleloch, R. Holmestad, and T. Tybell. "Electron energy loss spectroscopy investigation of $\mathrm{Pb}$ and Ti hybridization with $\mathrm{O}$ at the $\mathrm{PbTiO}_{3} / \mathrm{SrTiO}_{3}$ interface." In: J. Appl. Phys. 109.3 (Feb. 2011), p. 034104. ISSN: 00218979.

[326] Pau Torruella, Catalina Coll, Gemma Martín, Lluís López-Conesa, María Vila, Carlos Díaz-Guerra, María Varela, María Luisa Ruiz-González, Javier Piqueras, and Francesca Peiró. "Assessing Oxygen Vacancies in Bismuth Oxide through EELS Measurements and DFT Simulations." In: J. Phys. Chem. C 121.44 (2017), pp. 24809-24815.

[327] Michal Bajdich, Jens K Nørskov, and Aleksandra Vojvodic. "Surface energetics of alkaline-earth metal oxides: Trends in stability and adsorption of small molecules." In: Phys. Rev. B 91.15 (2015), p. 155401.

[328] Kozo Tanabe and Kaizaburo Saito. "The conversion of benzaldehyde into benzyl benzoate with alkaline earth metal oxide catalysts." In: Journal of Catalysis 35.2 (1974), pp. 247-255.

[329] Yoshio Ono and Hideshi Hattori. Solid base catalysis. Vol. 101. Springer Science \& Business Media, 2012. 
[330] Gianfranco Pacchioni, Josep M Ricart, and Francesc Illas. "Ab initio cluster model calculations on the chemisorption of $\mathrm{CO}_{2}$ and $\mathrm{SO}_{2}$ probe molecules on $\mathrm{MgO}$ and $\mathrm{CaO}$ (100) surfaces. A theoretical measure of oxide basicity." In: J. Am. Chem. Soc. 116.22 (1994), pp. 1015210158.

[331] Septimus HC Liang and Ian D Gay. "A 13 C solid-state NMR study of the chemisorption and decomposition of ethanol on $\mathrm{MgO} . "$ In: J. Catal. 101.2 (1986), pp. 293-300.

[332] Zdenek Dohnalek, Greg A Kimmel, Stephen A Joyce, Patrick Ayotte, R Scott Smith, and Bruce D Kay. "Physisorption of CO on the MgO(100) surface." In: J. Phys. Chem. B 105.18 (2001), pp. 3747-3751.

[333] M-C Wu, WS Oh, and DW Goodman. "Initial sticking probabilities of $\mathrm{Cu}$ vacuum deposited on ordered $\mathrm{Al}_{2} \mathrm{O}_{3}$ and MgO." In: Surf. Sci. 330.1 (1995), pp. 61-66.

[334] James C Phillips. "The chemical bond and solid-state physics." In: Physics Today 23.2 (1970), pp. 23-30.

[335] James A Van Vechten. "Quantum dielectric theory of electronegativity in covalent systems. I. Electronic dielectric constant." In: Phys. Rev. 182.3 (1969), p. 891.

[336] Gianfranco Pacchioni, Carmen Sousa, Francesc Illas, Fulvio Parmigiani, and Paul S Bagus. "Measures of ionicity of alkaline-earth oxides from the analysis of ab initio cluster wave functions." In: Phys. Rev. B 48.16 (1993), p. 11573.

[337] FMF De Groot, M Grioni, JC Fuggle, J Ghijsen, GA Sawatzky, and $\mathrm{H}$ Petersen. "Oxygen 1s x-ray-absorption edges of transition-metal oxides." In: Phys. Rev. B 40.8 (1989), p. 5715.

[338] Peter E Blöchl. "Projector augmented-wave method." In: Phys. Rev. B 50.24 (1994), p. 17953.

[339] Mathieu Taillefumier, Delphine Cabaret, Anne-Marie Flank, and Francesco Mauri. "X-ray absorption near-edge structure calculations with the pseudopotentials: Application to the K edge in diamond and $\alpha$-quartz." In: Physical Review B 66.19 (2002), p. 195107.

[340] Graeme Henkelman, Andri Arnaldsson, and Hannes Jónsson. "A fast and robust algorithm for Bader decomposition of charge density." In: Computational Materials Science 36.3 (2006), pp. 354-360.

[341] Georg Kresse and J Furthmüller. “Vienna ab-initio simulation package (vasp)." In: Vienna: Vienna University (2001). 
[342] Nicola Marzari, Arash A Mostofi, Jonathan R Yates, Ivo Souza, and David Vanderbilt. "Maximally localized Wannier functions: Theory and applications." In: Reviews of Modern Physics 84.4 (2012), p. 1419.

[343] Peter Blaha, Karlheinz Schwarz, Georg KH Madsen, Dieter Kvasnicka, Joachim Luitz, et al. "wien2k." In: An augmented plane wave+ local orbitals program for calculating crystal properties (2001).

[344] John P Perdew, Kieron Burke, and Matthias Ernzerhof. "Generalized gradient approximation made simple." In: Physical review letters 77.18 (1996), p. 3865.

[345] David Vanderbilt. "Soft self-consistent pseudopotentials in a generalized eigenvalue formalism." In: Physical review B 41.11 (1990), p. 7892.

[346] Y. Liang. XCH Pseudos. https : / / github . com/yufengliang / $\mathrm{XCH}_{-}$ pseudos.

[347] Satoshi SASAKI, Kiyoshi FUJINO, and Yoshio TAKÉUCHI. "X-ray determination of electron-density distributions in oxides, $\mathrm{MgO}, \mathrm{MnO}$, $\mathrm{CoO}$, and $\mathrm{NiO}$, and atomic scattering factors of their constituent atoms." In: Proceedings of the Japan Academy, Series B 55.2 (1979), pp. 43-48.

[348] Q Huang, O Chmaissem, JJ Capponi, C Chaillout, M Marezio, JL Tholence, and A Santoro. "Neutron powder diffraction study of the crystal structure of $\mathrm{HgBa}_{2} \mathrm{Ca}_{4} \mathrm{Cu}_{5} \mathrm{O}_{12}+\delta$ at room temperature and at 10 K." In: Physica C: Superconductivity 227.1-2 (1994), pp. 1-9.

[349] William Primak, Herman Kaufman, and Roland Ward. "X-ray diffraction studies of systems involved in the preparation of alkaline earth sulfide and selenide phosphorsı." In: Journal of the American Chemical Society 70.6 (1948), pp. 2043-2046.

[350] Walther Gerlach. “Die gitterstruktur der erdalkalioxyde." In: Zeitschrift für Physik 9.1 (1922), pp. 184-192.

[351] Norman Elliott. "A Redetermination of the Carbon-Oxygen Distance in Calcite and the Nitrogen-Oxygen Distance in Sodium Nitrate." In: Journal of the American Chemical Society 59.7 (1937), pp. 13801382.

[352] William R Busing and Henri A Levy. "Neutron diffraction study of calcium hydroxide." In: The Journal of Chemical Physics 26.3 (1957), pp. 563-568.

[353] Johan PR De Villiers. "Crystal structures of aragonite, strontianite, and witherite." In: American Mineralogist: Journal of Earth and Planetary Materials 56.5-6 (1971), pp. 758-767. 
[354] JS Ricci, RC Stevens, RK McMullan, and WT Klooster. "Structure of strontium hydroxide octahydrate, $\mathrm{Sr}(\mathrm{OH}) 2 \cdot 8 \mathrm{H}_{2} \mathrm{O}$, at 20, 100 and $200 \mathrm{~K}$ from neutron diffraction." In: Acta Crystallographica Section B: Structural Science 61.4 (2005), pp. 381-386.

[355] Paul Groth and Erich Kaiser. Zeitschrift Für Kristallographie, Kristallgeometrie, Kristallphysik, Kristallchemie. Vol. 9. Akademische Verlagsgesellschaft., 1884 .

[356] Alexandra Friedrich, Martin Kunz, and Emmanuelle Suard. “Temperaturedependent neutron powder diffraction study of the Ba (OD) 2 polymorphs: a new low-temperature phase." In: Acta Crystallographica Section B: Structural Science 57.6 (2001), pp. 747-758.

[357] Paolo Giannozzi, Stefano Baroni, Nicola Bonini, Matteo Calandra, Roberto Car, Carlo Cavazzoni, Davide Ceresoli, Guido L Chiarotti, Matteo Cococcioni, Ismaila Dabo, et al. "QUANTUM ESPRESSO: a modular and open-source software project for quantum simulations of materials." In: Journal of physics: Condensed matter 21.39 (2009), p. 395502.

[358] Eric L Shirley. "Optimal basis sets for detailed Brillouin-zone integrations." In: Physical Review B 54.23 (1996), p. 16464.

[359] AS Rao and RJ Kearney. "Logarithmic derivative reflectance spectra of $\mathrm{BaO}$ and SrO." In: physica status solidi (b) 95.1 (1979), pp. 243-250.

[360] Natalia V Skorodumova, Kersti Hermansson, and Börje Johansson. "Structural and electronic properties of the (100) surface and bulk of alkaline-earth metal oxides." In: Physical Review B 72.12 (2005), p. 125414 .

[361] Vladimir A Sashin, Helen E Dorsett, Mohammad A Bolorizadeh, and Michael J Ford. "The valence band structures of $\mathrm{BeO}, \mathrm{MgO}$, and CaO." In: The Journal of Chemical Physics 113.18 (2000), pp. 8175-8182.

[362] Th Lindner, H Sauer, W Engel, and K Kambe. "Near-edge structure in electron-energy-loss spectra of MgO." In: Phys. Rev. B 33.1 (1986), p. 22.

[363] Shun-ichi Nakai, Tsutomu Mitsuishi, Hidenao Sugawara, Hideki Maezawa, Tokuo Matsukawa, Shichiro Mitani, Kazuo Yamasaki, and Takashi Fujikawa. "Oxygen K x-ray-absorption near-edge structure of alkalineearth-metal and 3d-transition-metal oxides." In: Phys. Rev. B 36.17 (1987), p. 9241. 
[364] JosÉ Luis Pascual and Lars GM Pettersson. "Cluster modelling of O (1s) core excitons at the (100) surface of alkaline-earth oxides." In: Molecular Physics 101.1-2 (2003), pp. 255-265.

[365] HO Moltaji, JP Buban, JA Zaborac, and ND Browning. "Simulating the oxygen K-edge spectrum from grain boundaries in ceramic oxides using the multiple scattering methodology." In: Micron 31.4 (2000), pp. 381-399.

[366] Xudong Weng and Peter Rez. "Multiple-scattering approach to oxygen $\mathrm{K}$ near-edge structures in electron-energy-loss spectroscopy of alkaline earths." In: Phys. Rev. B 39.11 (1989), p. 7405.

[367] Javier Carrasco, Francesc Illas, and Nuria Lopez. "Dynamic ion pairs in the adsorption of isolated water molecules on alkaline-earth oxide (O01) surfaces." In: Physical review letters 100.1 (2008), p. 016101.

[368] Diego Alvarez and J Carlos Abanades. "Determination of the critical product layer thickness in the reaction of $\mathrm{CaO}$ with $\mathrm{CO} 2 . "$ In: Industrial \& engineering chemistry research 44.15 (2005), pp. 5608-5615.

[369] Teruyasu Mizoguchi, Isao Tanaka, Masato Yoshiya, Fumiyasu Oba, Kazuyoshi Ogasawara, and Hirohiko Adachi. "Core-hole effects on theoretical electron-energy-loss near-edge structure and near-edge $\mathrm{x}$ ray absorption fine structure of MgO." In: Phys. Rev. B 61.3 (2000), p. 2180.

[370] Xuelong Wang, Xiulin Fan, Xiqian Yu, Seongmin Bak, Zulipiya Shadike, Iradwikanari Waluyo, Adrian Hunt, Sanjaya D Senanayake, Hong Li, Liquan Chen, et al. "The Role of Electron Localization in Covalency and Electrochemical Properties of Lithium-Ion Battery Cathode Materials." In: Advanced Functional Materials (2020), p. 2001633.

[371] Shang-Peng Gao, Chris J Pickard, Alexander Perlov, and Victor Milman. "Core-level spectroscopy calculation and the plane wave pseudopotential method." In: Journal of Physics: Condensed Matter 21.10 (2009), p. 104203.

[372] Paul S Bagus, Francesc Illas, Gianfranco Pacchioni, and Fulvio Parmigiani. "Mechanisms responsible for chemical shifts of core-level binding energies and their relationship to chemical bonding." In: Journal of electron spectroscopy and related phenomena 100.1-3 (1999), pp. 215236. 
[373] Gianfranco Pacchioni and Paul S Bagus. "Theoretical analysis of the $\mathrm{O}$ (1s) binding-energy shifts in alkaline-earth oxides: Chemical or electrostatic contributions." In: Physical Review B 50.4 (1994), p. 2576.

[374] Tery L Barr and Ying Li Liu. "An X-ray photoelectron spectroscopy study of the valence band structure of indium oxides." In: Journal of Physics and Chemistry of Solids 50.7 (1989), pp. 657-664.

[375] Gabriel P López, David G Castner, and Buddy D Ratner. "XPS O Is binding energies for polymers containing hydroxyl, ether, ketone and ester groups." In: Surface and interface analysis 17.5 (1991), pp. 267-272.

[376] Geert Silversmit, Diederik Depla, Hilde Poelman, Guy B Marin, and Roger De Gryse. "Determination of the V2p XPS binding energies for different vanadium oxidation states ( $\mathrm{V}_{5}+$ to $\left.\mathrm{Vo}+\right)$." In: Journal of Electron Spectroscopy and Related Phenomena 135.2-3 (2004), pp. 167175 .

[377] Ignacio J Villar-Garcia, Emily F Smith, Alasdair W Taylor, Fulian Qiu, Kevin RJ Lovelock, Robert G Jones, and Peter Licence. "Charging of ionic liquid surfaces under X-ray irradiation: the measurement of absolute binding energies by XPS." In: Physical Chemistry Chemical Physics 13.7 (2011), pp. 2797-2808.

[378] Nils Måtensson and Anders Nilsson. "On the origin of core-level binding energy shifts." In: Journal of electron spectroscopy and related phenomena 75 (1995), pp. 209-223.

[379] MI Sosulnikov and Yu A Teterin. "X-ray photoelectron studies of Ca, $\mathrm{Sr}$ and Ba and their oxides and carbonates." In: Journal of electron spectroscopy and related phenomena 59.2 (1992), pp. 111-126.

[380] TL Barr and CR Brundle. "Bonding and electronic structure in high-T c superconducting oxides: A case for the importance of the cations." In: Physical Review B 46.14 (1992), p. 9199.

[381] Alan Herries Wilson. "The theory of electronic semi-conductors." In: Proc. R. Soc. London, Ser. A 133.822 (1931), pp. 458-491.

[382] CNR Rao. "Transition metal oxides." In: Annu. Rev. Phys. Chem. 40.1 (1989), pp. 291-326.

[383] FMF De Groot, Jl Faber, JJM Michiels, MT Czyżyk, M Abbate, and JC Fuggle. "Oxygen is x-ray absorption of tetravalent titanium oxides: A comparison with single-particle calculations." In: Phys. Rev. B 48.4 (1993), p. 2074. 
[384] SP Cramer, FMF DeGroot, Y Ma, CT Chen, F Sette, CA Kipke, DM Eichhorn, MK Chan, and WH Armstrong. "Ligand field strengths and oxidation states from manganese L-edge spectroscopy." In: J. Am. Chem. Soc. 113.21 (1991), pp. 7937-7940.

[385] Jin Suntivich, Wesley T Hong, Yueh-Lin Lee, James M Rondinelli, Wanli Yang, John B Goodenough, Bogdan Dabrowski, John W Freeland, and Yang Shao-Horn. "Estimating hybridization of transition metal and oxygen states in perovskites from $\mathrm{O}$ K-edge x-ray absorption spectroscopy." In: J. Phys. Chem. C 118.4 (2014), pp. 1856-1863.

[386] Cécile Hébert, Marc Willinger, Dang Sheng Su, Peter Pongratz, Peter Schattschneider, and Robert Schlögl. "Oxygen K-edge in vanadium oxides: simulations and experiments." In: Eur. Phys. J. B 28.4 (2002), pp. 407-414.

[387] H Kurata, E Lefevre, C Colliex, and R Brydson. "Electron-energy-loss near-edge structures in the oxygen K-edge spectra of transition-metal oxides." In: Phys. Rev. B 47.20 (1993), p. 13763.

[388] ZY Wu, S Gota, F Jollet, M Pollak, M Gautier-Soyer, and CR Natoli. "Characterization of iron oxides by x-ray absorption at the oxygen $\mathrm{K}$ edgeusing a full multiple-scattering approach." In: Phys. Rev. B 55.4 (1997), p. 2570.

[389] Christoph Mitterbauer, Gerald Kothleitner, Werner Grogger, H Zandbergen, B Freitag, P Tiemeijer, and F Hofer. "Electron energy-loss near-edge structures of $3 \mathrm{~d}$ transition metal oxides recorded at highenergy resolution." In: Ultramicroscopy 96.3-4 (2003), pp. 469-480.

[390] B Gilbert, BH Frazer, A Belz, PG Conrad, KH Nealson, D Haskel, JC Lang, G Srajer, and G De Stasio. "Multiple scattering calculations of bonding and X-ray absorption spectroscopy of manganese oxides." In: J. Phys. Chem. A 107.16 (2003), pp. 2839-2847.

[391] Stephan Schmidt and Dieter Schmeißer. "Electronic structure of cobaltnickel mixed oxides." In: Solid State Ionics 225 (2012), pp. 737-741.

[392] V Kanchana, G Vaitheeswaran, and M Alouani. "Calculated electronic structure and x-ray magnetic circular dichroism of $\mathrm{CrO}_{2}$." In: J. Phys.: Condens. Matter 18.22 (2006), p. 5155.

[393] M. A. Peña and J. L. G. Fierro. "Chemical Structures and Performance of Perovskite Oxides." In: Chem. Rev. 101.7 (July 2001), pp. 1981-2018. ISSN: 0009-2665. 
[394] Natalia Palina, Anil Annadi, Teguh Citra Asmara, Caozheng Diao, Xiaojiang Yu, Mark BH Breese, T Venkatesan, and Andrivo Rusydi. "Electronic defect states at the $\mathrm{LaAlO}_{3} / \mathrm{SrTiO}_{3}$ heterointerface revealed by O K-edge X-ray absorption spectroscopy." In: Phys. Chem. Chem. Phys. 18.20 (2016), pp. 13844-13851.

[395] B Chen et al. "Effects of rare-earth size on the electronic structure of $\mathrm{La}_{1-x} \mathrm{Lu}_{x} \mathrm{VO}_{3} . "$ In: J. Phys.: Condens. Matter 27.10 (2015), p. 105503.

[396] T. Mizokawa, A. Fujimori, H. Namatame, Y. Takeda, and M. Takano. "Electronic structure of tetragonal $\mathrm{LaCuO}_{3}$ studied by photoemission and x-ray-absorption spectroscopy." en. In: Phys. Rev. B 57.16 (Apr. 1998), pp. 9550-9556. ISSN: 0163-1829, 1095-3795.

[397] Rik Brydson, LAJ Garvie, AJ Craven, Hermann Sauer, Ferdinand Hofer, and G Cressey. " $\mathrm{L}_{2,3}$ edges of tetrahedrally coordinated do transition-metal oxyanions $\mathrm{XO}_{4}^{\mathfrak{n}-}$." In: J. Phys.: Condens. Matter 5.50 (1993), p. 9379.

[398] Stefan G Minasian, Jason M Keith, Enrique R Batista, Kevin S Boland, Joseph A Bradley, Scott R Daly, Stosh A Kozimor, Wayne W Lukens, Richard L Martin, and Dennis Nordlund. "Covalency in metal-oxygen multiple bonds evaluated using oxygen K-edge spectroscopy and electronic structure theory." In: J. Am. Chem. Soc. 135.5 (2013), pp. 18641871.

[399] Joseph A. Bradley et al. "Experimental and theoretical comparison of the $\mathrm{O} \mathrm{K}$-edge nonresonant inelastic X-ray scattering and X-ray absorption spectra of $\mathrm{NaReO}_{4}$." en. In: J. Am. Chem. Soc. 132.39 (Oct. 2010), pp. 13914-13921. ISSN: 0002-7863, 1520-5126. (Visited on 10/18/2019).

[40o] A Fujimori, I Hase, M Nakamura, H Namatame, Y Fujishima, Y Tokura, M Abbate, Frank MF de Groot, MT Czyzyk, and JC Fuggle. "Doping-induced changes in the electronic structure of $(\mathrm{La}, \mathrm{Sr}) \mathrm{TiO}_{3}$ : Limitation of the one-electron rigid-band model and the Hubbard model." In: Phys. Rev. B 46.15 (1992), p. 9841.

[401] LA Grunes, RD Leapman, CN Wilker, R Hoffmann, and AB Kunz. "Oxygen K near-edge fine structure: An electron-energy-loss investigation with comparisons to new theory for selected $3 \mathrm{~d}$ Transitionmetal oxides." In: Phys. Rev. B 25.12 (1982), p. 7157.

[402] R Brydson, H Sauer, W Engel, JM Thomass, E Zeitler, N Kosugi, and $\mathrm{H}$ Kuroda. "Electron energy loss and X-ray absorption spectroscopy of rutile and anatase: a test of structural sensitivity." In: J. Phys.: Condens. Matter 1.4 (1989), p. 797. 
[403] Rik Brydson, Hermann Sauer, Wilfried Engel, and Ferdinand Hofer. "Electron energy-loss near-edge structures at the oxygen K edges of titanium (IV) oxygen compounds." In: J. Phys.: Condens. Matter 4.13 (1992), p. 3429.

[404] PA Van Aken, B Liebscher, and VJ Styrsa. "Core level electron energyloss spectra of minerals: pre-edge fine structures at the oxygen Kedge." In: Phys. Chem. Miner. 25.7 (1998), pp. 494-498.

[405] Susanne Stemmer, SK Streiffer, Nigel D Browning, and Angus I Kingon. "Accommodation of nonstoichiometry in (100) fiber-textured (Ba,Sr) $\mathrm{TiO}_{3}$ thin films grown by chemical vapor deposition." In: Appl. Phys. Lett. 74.17 (1999), pp. 2432-2434.

[406] Jan Torgersen, Shinjita Acharya, Anup Lal Dadlani, Ioannis Petousis, Yongmin Kim, Orlando Trejo, Dennis Nordlund, and Fritz B Prinz. "Relating electronic and geometric structure of atomic layer deposited $\mathrm{BaTiO}_{3}$ to its electrical properties." In: J. Phys. Chem. Lett. 7.8 (2016), pp. 1428-1433.

[407] MP Kocher, DA Muller, and P Rez. "The oxygen K edge in strontium titanate and lanthanum titanate." In: Microscopy and Microanalysis 9.So2 (2003), pp. 842-843.

[408] Yufeng Liang, John Vinson, Sri Pemmaraju, Walter S Drisdell, Eric L Shirley, and David Prendergast. "Accurate X-ray spectral predictions: An advanced self-consistent-field approach inspired by many-body perturbation theory." In: Phys. Rev. Lett. 118.9 (2017), p. 096402.

[409] MW Haverkort, Z Hu, Arata Tanaka, G Ghiringhelli, H Roth, M Cwik, T Lorenz, C Schüßler-Langeheine, SV Streltsov, and AS Mylnikova. "Determination of the orbital moment and crystal-field splitting in $\mathrm{LaTiO}_{3}$." In: Phys. Rev. Lett. 94.5 (2005), p. 056401.

[410] M Abbate, H Pen, MT Czyżyk, FMF De Groot, JC Fuggle, YJ Ma, CT Chen, F Sette, A Fujimori, and Y Ueda. "Soft X-ray absorption spectroscopy of vanadium oxides." In: J. Electron Spectrosc. Relat. Phenom. 62.1-2 (1993), pp. 185-195.

[411] E Goering, O Mu, and S Horn. "Angular dependent soft x-ray absorption spectroscopy of vanadium oxides." In: Phys. B 194 (1994), pp. 1217-1218.

[412] J-H Park, LH Tjeng, Arata Tanaka, JW Allen, CT Chen, P Metcalf, JM Honig, FMF de Groot, and GA Sawatzky. "Spin and orbital occu- 
pation and phase transitions in $\mathrm{V}_{2} \mathrm{O}_{3}$." In: Phys. Rev. B 61.17 (2000), p. 11506.

[413] DJ Huang, LH Tjeng, J Chen, CF Chang, WP Wu, AD Rata, T Hibma, SC Chung, S-G Shyu, and C-C Wu. "Electron correlation effects in half-metallic transition metal oxides." In: Surf. Rev. Lett. 9.02 (2002), pp. 1007-1015.

[414] M Abbate, FMF De Groot, JC Fuggle, YJ Ma, CT Chen, F Sette, A Fujimori, Y Ueda, and K Kosuge. "Soft-x-ray-absorption studies of the electronic-structure changes through the $\mathrm{VO}_{2}$ phase transition." In: Phys. Rev. B 43.9 (1991), p. 7263.

[415] Hiroyuki Abe, Masami Terauchi, Michiyoshi Tanaka, Shik Shin, and Yutaka Ueda. "Electron energy-loss spectroscopy study of the metalinsulator transition in $\mathrm{VO}_{2}$." In: Jpn. J. Appl. Phys. 36.1R (1997), p. 165.

[416] Nicola Pinna, Marc Willinger, Klaus Weiss, Joachim Urban, and Robert Schlögl. "Local structure of nanoscopic materials: $\mathrm{V}_{2} \mathrm{O}_{5}$ nanorods and nanowires." In: Nano Lett. 3.8 (2003), pp. 1131-1134.

[417] Dimitrios Maganas, Michael Roemelt, Michael Hävecker, Annette Trunschke, Axel Knop-Gericke, Robert Schlögl, and Frank Neese. "First principles calculations of the structure and V L-edge X-ray absorption spectra of $\mathrm{V}_{2} \mathrm{O}_{5}$ using local pair natural orbital coupled cluster theory and spin-orbit coupled configuration interaction approaches." In: Phys. Chem. Chem. Phys. 15.19 (2013), pp. 7260-7276.

[418] Hyun Chul Choi, Young Mee Jung, and Seung Bin Kim. "Characterization of the electrochemical reactions in the $\mathrm{Li}_{1+\chi} \mathrm{V}_{3} \mathrm{O}_{8} / \mathrm{Li}$ cell by soft X-ray absorption spectroscopy and two-dimensional correlation analysis." In: Appl. Spectrosc. 57.8 (2003), pp. 984-990.

[419] Marc Georg Willinger, Dang Sheng Su, and Robert Schlögl. "Electronic structure of $\beta-\mathrm{VOPO}_{4}$." In: Phys. Rev. B 71.15 (2005), p. 155118.

[420] C Ma, HX Yang, ZA Li, Y Ueda, and JQ Li. "Charge disproportionation in quasi-one-dimensional vanadium oxides." In: Solid State Commun. 146.1-2 (2008), pp. 30-34.

[421] S Kalavathi, S Amirthapandian, Sharat Chandra, P Ch Sahu, and HK Sahu. "Valence state, hybridization and electronic band structure in the charge ordered $\mathrm{AlV}_{2} \mathrm{O}_{4}$." In: J. Phys.: Condens. Matter 26.1 (2013), p. 015601 . 
[422] Vedran Jovic, J Laverock, AJE Rettie, J-S Zhou, CB Mullins, VR Singh, B Lamoureux, D Wilson, T-Y Su, and B Jovic. "Soft X-ray spectroscopic studies of the electronic structure of $\mathrm{M}: \mathrm{BiVO}_{4}(\mathrm{M}=\mathrm{Mo}, \mathrm{W})$ single crystals." In: J. Mater. Chem. A 3.47 (2015), pp. 23743-23753.

[423] LFJ Piper, A DeMasi, SW Cho, ARH Preston, J Laverock, KE Smith, KG West, JW Lu, and SA Wolf. "Soft x-ray spectroscopic study of the ferromagnetic insulator $\mathrm{V}(\mathrm{Cr}) \mathrm{O}_{2}$." In: Phys. Rev. B 82.23 (2010), p. 235103.

[424] "XASEELS database." In: www.anorg.chem.uu.nl/xaseels/XASEELSintro.html (2019).

[425] Oana Corina Rogojanu, GA Sawatzky, and LH Tjeng. "Stabilizing $\mathrm{CrO}$ by epitaxial growth." PhD thesis. University Library Groningen][Host], 2002.

[426] Th Schedel-Niedrig. "X-Ray absorption spectroscopy: sensitive characterization of (model-) catalysts with the electron yield technique." In: J. Anal. Chem. 361.6-7 (1998), pp. 680-682.

[427] P Diaz-Carrasco, P Moreau, Dominique Guyomard, A Kuhn, and F Garcia-Alvarado. "Electron energy loss spectroscopy analysis of lithium deintercalated $\mathrm{Li}_{5 / 3-\chi} \mathrm{Ti}_{7 / 3} \mathrm{CrO}_{7}$." In: J. Phys. Chem. Solids 67.56 (2006), pp. 1295-1298.

[428] SO Kucheyev, B Sadigh, TF Baumann, YM Wang, TE Felter, T Van Buuren, AE Gash, JH Satcher Jr, and AV Hamza. “Electronic structure of chromia aerogels from soft x-ray absorption spectroscopy." In: $J$. Appl. Phys. 101.12 (2007), p. 124315.

[429] FT Docherty, AJ Craven, DW McComb, and J Skakle. "ELNES investigations of the oxygen K-edge in spinels." In: Ultramicroscopy 86.3-4 (2001), pp. 273-288.

[430] DD Sarma, K Maiti, E Vescovo, C Carbone, W Eberhardt, O Rader, and W Gudat. "Investigation of hole-doped insulating ( $\mathrm{La}, \mathrm{Sr}) \mathrm{CrO}_{3}$ by soft-x-ray absorption spectroscopy." In: Phys. Rev. B 53.20 (1996), p. 13369.

[431] Eberhard Goering, A Bayer, S Gold, Gisela Schütz, M Rabe, Ulrich Rüdiger, and Gernot Güntherodt. "Direct correlation of $\mathrm{Cr}$ 3d orbital polarization and $\mathrm{O} \mathrm{K}$-edge $\mathrm{X}$-ray magnetic circular dichroism of epitaxial $\mathrm{CrO}_{2}$ films." In: Europhys. Lett. 58.6 (2002), p. 906. 
[432] Akihiro Koide and Toshihiko Yokoyama. "Effects of spin-orbit interaction in chromium on oxygen K-edge x-ray magnetic circular dichroism spectra in $\mathrm{CrO}_{2}$." In: Phys. Rev. B 96.14 (2017), p. 144419.

[433] Letizia Monico, Geert Van der Snickt, Koen Janssens, Wout De Nolf, Costanza Miliani, Johan Verbeeck, He Tian, Haiyan Tan, Joris Dik, and Marie Radepont. "Degradation process of lead chromate in paintings by Vincent van Gogh studied by means of synchrotron X-ray spectromicroscopy and related methods. 1. Artificially aged model samples." In: Anal. Chem. 83.4 (2011), pp. 1214-1223.

[434] L Soriano, M Abbate, FMF De Groot, D Alders, JC Fuggle, S Hofmann, $\mathrm{H}$ Petersen, and $\mathrm{W}$ Braun. "Chemical analysis of passivated and oxidized layers on $\mathrm{FeCr}$ and FeTi alloys by soft x-ray absorption spectroscopy." In: Surf. Interface Anal. 20.1 (1993), pp. 21-26.

[435] María Francisca López, A Gutierrez, CL Torres, and JM Bastidas. "Soft x-ray absorption spectroscopy study of electrochemically formed passive layers on AISI 304 and stainless steels." In: J. Mater. Res. 14.3 (1999), pp. 763-770.

[436] James H Paterson and Ondrej L Krivanek. "ELNES of 3d transitionmetal oxides: II. Variations with oxidation state and crystal structure." In: Ultramicroscopy 32.4 (1990), pp. 319-325.

[437] Franciscus Martinus Frederikus de Groot. X-ray absorption of transition metal oxides. [S1: sn], 1991.

[438] Hiroki Kurata and Christian Colliex. "Electron-energy-loss core-edge structures in manganese oxides." In: Phys. Rev. B 48.4 (1993), p. 2102.

[439] EZ Kurmaev, RG Wilks, A Moewes, LD Finkelstein, SN Shamin, and J Kuneš. "Oxygen x-ray emission and absorption spectra as a probe of the electronic structure of strongly correlated oxides." In: Phys. Rev. B 77.16 (2008), p. 165127.

[440] Joke Hadermann, Artem M Abakumov, Tyche Perkisas, Hans D'Hondt, Haiyan Tan, Johan Verbeeck, Vladimir P Filonenko, Evgeny V Antipov, and Gustaaf Van Tendeloo. "New perovskite-based manganite $\mathrm{Pb}_{2} \mathrm{Mn}_{2} \mathrm{O}_{5}$." In: J. Phys.: Condens. Matter 183.9 (2010), pp. 2190-2195.

[441] Ruimin Qiao, Timothy Chin, Stephen J Harris, Shishen Yan, and Wanli Yang. "Spectroscopic fingerprints of valence and spin states in manganese oxides and fluorides." In: Curr. Appl. Phys. 13.3 (2013), pp. 544548 . 
[442] Marcel Risch, Kelsey A Stoerzinger, Binghong Han, Tom Z Regier, Derek Peak, Sayed Youssef Sayed, Chao Wei, Zhichuan Xu, and Yang Shao-Horn. "Redox processes of manganese oxide in catalyzing oxygen evolution and reduction: An in situ soft X-ray absorption spectroscopy study." In: J. Phys. Chem. C 121.33 (2017), pp. 17682-17692.

[443] Yifan Ye, Mukes Kapilashrami, Cheng-Hao Chuang, Yi-sheng Liu, Per-Anders Glans, and Jinghua Guo. "X-ray spectroscopies studies of the $3 \mathrm{~d}$ transition metal oxides and applications of photocatalysis." In: MRS Communications 7.1 (2017), pp. 53-66.

[444] M Abbate, Frank MF de Groot, JC Fuggle, A Fujimori, O Strebel, F Lopez, M Domke, G Kaindl, GA Sawatzky, and M Takano. “Controlledvalence properties of $(\mathrm{La}, \mathrm{Sr}) \mathrm{FeO}_{3}$ and $(\mathrm{La}, \mathrm{Sr}) \mathrm{MnO}_{3}$ studied by soft-xray absorption spectroscopy." In: Phys. Rev. B 46.8 (1992), p. 4511.

[445] Sara Lafuerza, G Subías, Joaquín García, S Di Matteo, Javier Blasco, V Cuartero, and Calogero R Natoli. "Origin of the pre-peak features in the oxygen K-edge x-ray absorption spectra of $\mathrm{LaFeO}_{3}$ and $\mathrm{LaMnO}_{3}$ studied by Ga substitution of the transition metal ion." In: J. Phys.: Condens. Matter 23.32 (2011), p. 325601.

[446] O Toulemonde, F Millange, F Studer, B Raveau, JH Park, and CT Chen. "Changes in the Jahn-Teller distortion at the metal-insulator transition in CMR manganites." In: J. Phys.: Condens. Matter 11.1 (1999), p. 109 .

[447] A Galdi, C Aruta, P Orgiani, C Adamo, V Bisogni, NB Brookes, G Ghiringhelli, DG Schlom, P Thakur, and L Maritato. "Electronic band redistribution probed by oxygen absorption spectra of $\left(\mathrm{SrMnO}_{3}\right) \mathrm{n} /\left(\mathrm{LaMnO}_{3}\right) 2 \mathrm{n}$ superlattices." In: Phys. Rev. B 85.12 (2012), p. 125129.

[448] EP Domashevskaya, SA Storozhilov, S Yu Turishchev, VM Kashkarov, VA Terekhov, OV Stogneĭ, Yu E Kalinin, AV Sitnikov, and SL Molodtsov. "XANES and USXES studies of interatomic interactions in $\left(\mathrm{Co}_{41} \mathrm{Fe}_{39} \mathrm{~B}_{20}\right)_{x}\left(\mathrm{SiO}_{2}\right)_{1-\chi}$ nanocomposites." In: Phys. Solid State 50.1 (2008), pp. 139-145.

[449] Z Hu, C Grazioli, M Knupfer, MS Golden, J Fink, Priya Mahadevan, Ashwani Kumar, Sugata Ray, DD Sarma, and SA Warda. "Difference in spin state and covalence between $\mathrm{La}_{1-\chi} \mathrm{Sr}_{\chi} \mathrm{CoO}_{3}$ and $\mathrm{La}_{2-\chi} \mathrm{Sr}_{\chi} \mathrm{Li}_{0.5} \mathrm{Co}_{0.5} \mathrm{O}_{4}$." In: J. Alloys Compd. 343.1-2 (2002), pp. 5-13.

[45o] ZY Wu, M Benfatto, M Pedio, R Cimino, S Mobilio, SR Barman, $\mathrm{K}$ Maiti, and DD Sarma. "Theoretical analysis of x-ray-absorption near-edge fine structure at the $\mathrm{O}$ and metal $\mathrm{K}$ edges of $\mathrm{LaFeO}_{3}$ and $\mathrm{LaCoO}_{3} . "$ In: Phys. Rev. B 56.4 (1997), p. 2228. 
[451] AS Harvey, Z Yang, A Infortuna, D Beckel, JA Purton, and Ludwig J Gauckler. "Development of electron holes across the temperatureinduced semiconductor-metal transition in $\mathrm{Ba}_{1-x} \mathrm{Sr}_{x} \mathrm{Co}_{1-y} \mathrm{Fe}_{y} \mathrm{O}_{3-\delta}(\mathrm{x}$, $\mathrm{y}=0.2-0.8)$ : a soft $\mathrm{x}$-ray absorption spectroscopy study." In: J. Phys.: Condens. Matter 21.1 (2008), p. 015801.

[452] Wanchao Zheng, Dongxing Zheng, Dong Li, Peng Li, Linxing Zhang, Junlu Gong, Xin Pang, Chao Jin, Xixiang Zhang, and Haili Bai. "Strain control of phase transition and magnetic property in multiferroic $\mathrm{BiFeO}_{3}$ thin films." In: Thin Solid Films 695 (2020), p. 137741.

[453] J Van Elp, JL Wieland, H Eskes, P Kuiper, GA Sawatzky, FMF De Groot, and TS Turner. "Electronic structure of $\mathrm{CoO}$, Li-doped $\mathrm{CoO}$ and $\mathrm{LiCoO}_{2}$." In: Phys. Rev. B 44.12 (1991), p. 6090.

[454] FMF De Groot, M Abbate, J Van Elp, GA Sawatzky, YJ Ma, CT Chen, and F Sette. "Oxygen 1s and cobalt 2p X-ray absorption of cobalt oxides." In: J. Phys.: Condens. Matter 5.14 (1993), p. 2277.

[455] Z Hu, G Kaindl, A Hayer, and D Reinen. "Kantennahe RöntgenfeinstrukturUntersuchungen an Oxidkeramiken von Chrom, Cobalt und Nickel in verschiedenen Oxidationsstufen und deren bindungschemische Interpretation." In: Z. Anorg. Allg. Chem. 627.12 (2001), pp. 2647-2653.

[456] Won-Sub Yoon, Kwang-Bum Kim, Min-Gyu Kim, Min-Kyu Lee, HyunJoon Shin, Jay-Min Lee, Jae-Sung Lee, and Chul-Hyun Yo. "Oxygen contribution on Li-ion intercalation- deintercalation in $\mathrm{LiCoO}_{2}$ investigated by $\mathrm{O}$ K-edge and Co L-edge X-ray absorption spectroscopy." In: J. Phys. Chem. B 106.10 (2002), pp. 2526-2532.

[457] Ching-Hsiang Chen, Bing-Joe Hwang, Chun-Yu Chen, Shao-Kang Hu, Jing-Ming Chen, Hwo-Shuenn Sheu, and Jyh-Fu Lee. "Soft X-ray absorption spectroscopy studies on the chemically delithiated commercial $\mathrm{LiCoO}_{2}$ cathode material." In: J. Power Sources 174.2 (2007), pp.938943 .

[458] Amélie Juhin, Frank De Groot, György Vankó, Matteo Calandra, and Christian Brouder. "Angular dependence of core hole screening in $\mathrm{LiCoO}_{2}$ : A DFT+U calculation of the oxygen and cobalt K-edge x-ray absorption spectra." In: Phys. Rev. B 81.11 (2010), p. 115115.

[459] Z Hu, Hua Wu, MW Haverkort, HH Hsieh, H-J Lin, T Lorenz, J Baier, A Reichl, I Bonn, and C Felser. "Different Look at the Spin State of $\mathrm{Co}_{3}{ }^{+}$Ions in a $\mathrm{CoO}_{5}$ Pyramidal Coordination." In: Phys. Rev. Lett. 92.20 (2004), p. 207402. 
[46o] M Abbate, JC Fuggle, A Fujimori, LH Tjeng, CT Chen, R Potze, GA Sawatzky, H Eisaki, and S Uchida. "Electronic structure and spinstate transition of $\mathrm{LaCoO}_{3}$." In: Phys. Rev. B 47.24 (1993), p. 16124.

[461] Johannes Simböck, M Ghiasi, Simon Schönebaum, Ulrich Simon, Frank MF de Groot, and Regina Palkovits. "Electronic parameters in cobaltbased perovskite-type oxides as descriptors for chemocatalytic reactions." In: Nature communications 11.1 (2020), pp. 1-10.

[462] AR Moodenbaugh, B Nielsen, Sharadha Sambasivan, Daniel A Fischer, T Friessnegg, S Aggarwal, R Ramesh, and RL Pfeffer. "Holestate density of $(\mathrm{La}, \mathrm{Sr}) \mathrm{CoO}_{3}-\delta$ across the insulator/metal phase boundary." In: Phys. Rev. B 61.8 (2000), p. 5666.

[463] I Davoli, A Marcelli, A Bianconi, M Tomellini, and M Fanfoni. "Multielectron configurations in the $\mathrm{x}$-ray-absorption near-edge structure of $\mathrm{NiO}$ at the oxygen K threshold." In: Phys. Rev. B 33.4 (1986), p. 2979.

[464] H Kuhlenbeck, G Odörfer, R Jaeger, G Illing, M Menges, Th Mull, H-J Freund, M Pöhlchen, V Staemmler, and S Witzel. "Molecular adsorption on oxide surfaces: Electronic structure and orientation of $\mathrm{NO}$ on $\mathrm{NiO}(100) / \mathrm{Ni}(100)$ and on $\mathrm{NiO}(100)$ as determined from electron spectroscopies and ab initio cluster calculations." In: Phys. Rev. B 43.3 (1991), p. 1969.

[465] E Zschech, L Tröger, D Arvanitis, H Michaelis, U Grimm, and K Baberschke. "A study of the self-absorption effect in the fluorescence yield of $\mathrm{NiO}$ at the oxygen K-edge." In: Solid State Commun. 82.1 (1992), pp. 1-5.

[466] Hideyuki Kanda, Masato Yoshiya, Fumiyasu Oba, Kazuyoshi Ogasawara, Hirohiko Adachi, and Isao Tanaka. "Cluster calculation of oxygen K-edge electron-energy-loss near-edge structure of $\mathrm{NiO} . "$ In: Phys. Rev. B 58.15 (1998), p. 9693.

[467] Z Hu, G Kaindl, SA Warda, D Reinen, FMF De Groot, and BG Müller. "On the electronic structure of $\mathrm{Cu}$ (III) and $\mathrm{Ni}$ (III) in $\mathrm{La}_{2} \mathrm{Li}_{1 / 2} \mathrm{Cu}_{1 / 2} \mathrm{O}_{4}$, $\mathrm{Nd}_{2} \mathrm{Li}_{1 / 2} \mathrm{Ni}_{1 / 2} \mathrm{O}_{4}$, and $\mathrm{Cs}_{2} \mathrm{KCuF}_{6}$." In: Chemical physics $232.1-2$ (1998), pp. 63-74.

[468] L-C Duda, Thorsten Schmitt, Martin Magnuson, Johan Forsberg, Anders Olsson, Joseph Nordgren, K Okada, and A Kotani. "Resonant inelastic $\mathrm{x}$-ray scattering at the oxygen $\mathrm{K}$ resonance of $\mathrm{NiO}$ : Nonlocal charge transfer and double-singlet excitations." In: Phys. Rev. Lett. 96.6 (2006), p. 067402. 
[469] Z Hu, Chandan Mazumdar, G Kaindl, FMF De Groot, SA Warda, and D Reinen. "Valence electron distribution in $\mathrm{La}_{2} \mathrm{Li}_{1 / 2} \mathrm{Cu}_{1 / 2} \mathrm{O}_{4}, \mathrm{Nd}_{2} \mathrm{Li}_{1 / 2} \mathrm{Ni}_{1 / 2} \mathrm{O}$ and $\mathrm{La}_{2} \mathrm{Li}_{1 / 2} \mathrm{Co}_{1 / 2} \mathrm{O}_{4}$." In: Chem. Phys. Lett. 297.3-4 (1998), pp. 321328.

[470] C Piamonteze, FMF De Groot, HCN Tolentino, AY Ramos, NE Massa, JA Alonso, and MJ Martínez-Lope. "Spin-orbit-induced mixed-spin ground state in $\mathrm{RNiO}_{3}$ perovskites probed by x-ray absorption spectroscopy: Insight into the metal-to-insulator transition." In: Phys. Rev. B 71.2 (2005), p. 020406.

[471] Takashi Nakamura, Ryo Oike, Yihan Ling, Yusuke Tamenori, and Koji Amezawa. "The determining factor for interstitial oxygen formation in Ruddlesden-Popper type $\mathrm{La}_{2} \mathrm{NiO}_{4}$-based oxides." In: Phys. Chem. Chem. Phys. 18.3 (2016), pp. 1564-1569.

[472] Z Hu, M Knupfer, M Kielwein, UK Rößler, MS Golden, J Fink, FMF de Groot, T Ito, K Oka, and G Kaindl. "The electronic structure of the doped one-dimensional transition metal oxide $\mathrm{Y}_{2-x} \mathrm{Ca}_{x} \mathrm{BaNiO}_{5}$ studied using X-ray absorption." In: Eur. Phys. J. B 26.4 (2002), pp. 449453.

[473] Alejandra B Gurevich, Brian E Bent, Andrew V Teplyakov, and Jingguang $\mathrm{G}$ Chen. "A NEXAFS investigation of the formation and decomposition of $\mathrm{CuO}$ and $\mathrm{Cu}_{2} \mathrm{O}$ thin films on $\mathrm{Cu}(100)$." In: Surf. Sci. 442.1 (1999), pp. L971-L976.

[474] Peng Jiang, David Prendergast, Ferenc Borondics, Soeren Porsgaard, Lisandro Giovanetti, Elzbieta Pach, John Newberg, Hendrik Bluhm, Flemming Besenbacher, and Miquel Salmeron. "Experimental and theoretical investigation of the electronic structure of $\mathrm{Cu}_{2} \mathrm{O}$ and $\mathrm{CuO}$ thin films on $\mathrm{Cu}$ (110) using x-ray photoelectron and absorption spectroscopy." In: J. Chem. Phys. 138.2 (2013), p. 024704.

[475] JP Hu, DJ Payne, RG Egdell, P-A Glans, T Learmonth, KE Smith, J Guo, and NM Harrison. "On-site interband excitations in resonant inelastic x-ray scattering from $\mathrm{Cu}_{2} \mathrm{O}$." In: Phys. Rev. B 77.15 (2008), p. 155115 .

[476] J Fink, N Nücker, E Pellegrin, H Romberg, M Alexander, and M Knupfer. "Electron energy-loss and x-ray absorption spectroscopy of cuprate superconductors and related compounds." In: J. Electron Spectrosc. Relat. Phenom. 66.3-4 (1994), pp. 395-452. 
[477] H Romberg, M Alexander, N Nücker, P Adelmann, and J Fink. “Electronic structure of the system $\mathrm{La}_{2-\chi} \mathrm{Sr}_{x} \mathrm{CuO}_{4+\delta}$." In: Phys. Rev. B 42.13 (1990), p. 8768.

[478] CT Chen, F Sette, Y Ma, MS Hybertsen, EB Stechel, WMC Foulkes, M Schulter, SW Cheong, AS Cooper, and LW Rupp Jr. "Electronic states in $\mathrm{La}_{2-\chi} \mathrm{Sr}_{x} \mathrm{CuO}_{4+\delta}$ probed by soft-X-ray absorption." In: Phys. Rev. Lett. 66.1 (1991), p. 104.

[479] Kenji Ishii, Takami Tohyama, Shun Asano, Kentaro Sato, Masaki Fujita, Shuichi Wakimoto, Kenji Tustsui, Shigetoshi Sota, Jun Miyawaki, Hideharu Niwa, et al. "Observation of momentum-dependent charge excitations in hole-doped cuprates using resonant inelastic x-ray scattering at the oxygen K edge." In: Phys. Rev. B 96.11 (2017), p. 115148.

[480] M Haruta, T Nagai, NR Lugg, MJ Neish, M Nagao, K Kurashima, LJ Allen, T Mizoguchi, and K Kimoto. "Atomic resolution chemical bond analysis of oxygen in $\mathrm{La}_{2} \mathrm{CuO}_{4}$." In: J. Appl. Phys. 114.8 (2013), p. 083712 .

[481] RJ Gaboriaud, M Jublot, F Paumier, and B Lacroix. "Phase transformations in $\mathrm{Y}_{2} \mathrm{O}_{3}$ thin films under swift Xe ions irradiation." In: Nucl. Instrum. Methods Phys. Res., Sect. B 310 (2013), pp. 6-9.

[482] L Soriano, M Abbate, JC Fuggle, MA Jimenez, JM Sanz, C Mythen, and HA Padmore. "The $\mathrm{O}$ is x-ray absorption spectra of transitionmetal oxides: The $\mathrm{TiO}_{2}-\mathrm{ZrO}_{2}-\mathrm{HfO}_{2}$ and $\mathrm{V}_{2} \mathrm{O}_{5}-\mathrm{Nb}_{2} \mathrm{O}_{5}-\mathrm{Ta}_{2} \mathrm{O}_{5}$ series." In: Solid State Commun. 87.8 (1993), pp. 699-703.

[483] S Ostanin, AJ Craven, DW McComb, D Vlachos, A Alavi, MW Finnis, and AT Paxton. "Effect of relaxation on the oxygen K-edge electron energy-loss near-edge structure in yttria-stabilized zirconia." In: Phys. Rev. B 62.22 (2000), p. 14728.

[484] MJ Olszta, J Wang, and EC Dickey. "Stoichiometry and valence measurements of niobium oxides using electron energy-loss spectroscopy." In: J. Microsc. 224.3 (2006), pp. 233-241.

[485] Guohua Wu, Tetsuhiro Sekiguchi, Yuji Baba, and Iwao Shimoyama. "X-ray absorption fine structure and photon-stimulated ion desorption from solid $\mathrm{MoO}_{3}$ at molybdenum $3 \mathrm{p}_{1 / 2}, 3 \mathrm{p}_{3 / 2}$ and oxygen is edges." In: Nucl. Instrum. Methods Phys. Res., Sect. B 245.2 (2006), pp. 406410. 
[486] Di Wang, Dang Sheng Su, and Robert Schlögl. "Electron beam induced transformation of $\mathrm{MoO}_{3}$ to $\mathrm{MoO}_{2}$ and a new phase MoO." In: Z. Anorg. Allg. Chem. 630.7 (2004), pp. 1007-1014.

[487] HM Tsai, PD Babu, CW Pao, JW Chiou, JC Jan, KP Krishna Kumar, FZ Chien, WF Pong, M-H Tsai, and C-H Chen. "Comparison of electronic structures of $\mathrm{RuO}_{2}$ and $\mathrm{IrO}_{2}$ nanorods investigated by x-ray absorption and scanning photoelectron microscopy." In: Appl. Phys. Lett. 90.4 (2007), p. 042108.

[488] A Travlos, N Boukos, G Apostolopoulos, and A Dimoulas. "Oxygen vacancy ordering in epitaxial layers of yttrium oxide on $\mathrm{Si}$ (OOI)." In: Appl. Phys. Lett. 82.23 (2003), pp. 4053-4055.

[489] M Malvestuto, R Carboni, F Boscherini, F d'Acapito, S Spiga, M Fanciulli, A Dimoulas, G Vellianitis, and G Mavrou. "X-ray absorption study of the growth of $\mathrm{Y}_{2} \mathrm{O}_{3}$ on Si (001)." In: Phys. Rev. B 71.7 (2005), p. 075318.

[490] Hidehiro Yoshida, Koji Morita, Byung-Nam Kim, Yoshio Sakka, and Takahisa Yamamoto. "Reduction in sintering temperature for flashsintering of yttria by nickel cation-doping." In: Acta Mater. 106 (2016), pp. 344-352.

[491] David W McComb. "Bonding and electronic structure in zirconia pseudopolymorphs investigated by electron energy-loss spectroscopy." In: Phys. Rev. B 54.10 (1996), p. 7094.

[492] Vladimir V Roddatis, Dang Sheng Su, Erich Beckmann, Friederike C Jentoft, Ulrike Braun, Jutta Kröhnert, and Robert Schlögl. "The structure of thin zirconia films obtained by self-assembled monolayer mediated deposition: TEM and HREM study." In: Surf. Coat. Technol. 151 (2002), pp. 63-66.

[493] G Lucovsky, Y Zhang, CC Fulton, Y Zou, RJ Nemanich, H Ade, and JL Whitten. "Final state effects in VUV and soft X-ray absorption spectra of transition metal oxides and silicate alloys: comparisons between experiment and ab initio calculations." In: J. Electron Spectrosc. Relat. Phenom. 144 (2005), pp. 917-919.

[494] Kwang Sik Jeong, Jinho Song, Donghyuck Lim, Hyungsub Kim, and Mann-Ho Cho. "Electronic structure of Ce-doped $\mathrm{ZrO}_{2}$ film: Study of DFT calculation and photoelectron spectroscopy." In: Appl. Sci. Converg. Technol . 25.1 (2016), pp. 19-24. 
[495] Aditya Sharma, Mayora Varshney, Keun Hwa Chae, Hyun Joon Shin, and Sung Ok Won. "Investigation on the local electronic/atomic structure properties using XANES/EXAFS and photocatalyst application of $(\mathrm{Zr}, \mathrm{Cu}) \mathrm{O}_{2}$." In: Curr. Appl. Phys. 16.10 (2016), pp. 1326-1333.

[496] Mayora Varshney, Aditya Sharma, Keun Hwa Chae, Shalendra Kumar, and Sung Ok Won. "Electronic structure and dielectric properties of $\mathrm{ZrO}_{2}-\mathrm{CeO}_{2}$ mixed oxides." In: J. Phys. Chem. Solids 119 (2018), pp. 242-250.

[497] V Srot, M Watanabe, C Scheu, PA Van Aken, U Salzberger, B Luerßen, J Janek, and M Rühle. "Characterization of chemical composition and electronic structure of $\mathrm{Pt} / \mathrm{YSZ}$ interfaces by analytical transmission electron microscopy." In: Solid State Ionics 181.35-36 (2010), pp. 16161622.

[498] Wilayat Khan, Sophia B Betzler, Ondrej Sipr, Jim Ciston, Peter Blaha, Christina Scheu, and Jan Minar. "Theoretical and experimental study on the optoelectronic properties of $\mathrm{Nb}_{3} \mathrm{O}_{7}(\mathrm{OH})$ and $\mathrm{Nb}_{2} \mathrm{O}_{5}$ photoelectrodes." In: J. Phys. Chem. C 120.41 (2016), pp. 23329-23338.

[499] J Purans, A Kuzmin, Ph Parent, and C Laffone. "Study of the electronic structure of rhenium and tungsten oxides on the O K-edge." In: Ionics 4.1-2 (1998), pp. 101-105.

[500] O Yu Khyzhun, T Strunskus, and Yu M Solonin. "XES, XPS and NEXAFS studies of the electronic structure of cubic $\mathrm{MoO}_{1} \cdot 9$ and $\mathrm{H}_{1} \cdot 63 \mathrm{MoO}_{3}$ thick films." In: J. Alloys Compd. $366.1-2$ (2004), pp. 5460.

[501] P Thakur, JC Cezar, NB Brookes, RJ Choudhary, DM Phase, KH Chae, and Ravi Kumar. "X-ray absorption and magnetic circular dichroism characterization of $\mathrm{MoFexO}_{2}(\mathrm{x}=\mathrm{O}-\mathrm{O} .05)$ thin films grown by pulsed laser ablation." In: Hyperfine Interact. 197.1-3 (2010), pp. 95-100.

[502] L Lajaunie, F Boucher, R Dessapt, and P Moreau. "Quantitative use of electron energy-loss spectroscopy Mo-M $\mathrm{M}_{2,3}$ edges for the study of molybdenum oxides." In: Ultramicroscopy 149 (2015), pp. 1-8.

[503] Robert Schlögl, Axel Knop-Gericke, Michael Hävecker, Ute Wild, Dietrich Frickel, Thorsten Ressler, Rolf E Jentoft, Julia Wienold, Gerhard Mestl, and Andreas Blume. "In situ analysis of metal-oxide systems used for selective oxidation catalysis: how essential is chemical complexity?" In: Topics in Catalysis 15.2-4 (2001), pp. 219-228. 
[504] J Purans, A Kuzmin, Ph Parent, and C Laffon. "X-ray absorption study of the electronic structure of tungsten and molybdenum oxides on the O K-edge." In: Electrochim. Acta 46.13-14 (2001), pp. 19731976.

[505] M Schmidt, TR Cummins, M Bürk, DH Lu, N Nücker, S Schuppler, and F Lichtenberg. "Nature of the electronic states in the layered perovskite noncuprate superconductor $\mathrm{Sr}_{2} \mathrm{RuO}_{4}$." In: Phys. Rev. B 53.22 (1996), R14761.

[506] Zhiwei Hu, H Von Lips, MS Golden, J Fink, G Kaindl, FMF De Groot, Stefan Ebbinghaus, and Armin Reller. "Multiplet effects in the Ru $\mathrm{L}_{2,3}$ x-ray-absorption spectra of Ru (IV) and Ru (V) compounds." In: Phys. Rev. B 61.8 (2000), p. 5262.

[507] JG Zhou, HT Fang, YF Hu, TK Sham, CX Wu, M Liu, and F Li. "Immobilization of $\mathrm{RuO}_{2}$ on carbon nanotube: An x-ray absorption nearedge structure study." In: J. Phys. Chem. C 113.24 (2009), pp. 1074710750.

[508] T Mizokawa, LH Tjeng, GA Sawatzky, G Ghiringhelli, Oscar Tjernberg, NB Brookes, H Fukazawa, S Nakatsuji, and Y Maeno. "Spinorbit coupling in the Mott insulator $\mathrm{Ca}_{2} \mathrm{RuO}_{4}$." In: Phys. Rev. Lett. 87.7 (2001), p. 077202.

[509] M Malvestuto, V Capogrosso, E Carleschi, L Galli, E Gorelov, E Pavarini, R Fittipaldi, F Forte, M Cuoco, and A Vecchione. "Nature of the apical and planar oxygen bonds in the $(\mathrm{Sr}, \mathrm{Ru}) \mathrm{O}_{3 \mathrm{n}+1}$ family $(\mathrm{n}=1,2,3) . "$ In: Phys. Rev. B 88.19 (2013), p. 195143.

[510] CG Fatuzzo, M Dantz, S Fatale, P Olalde-Velasco, NE Shaik, B Dalla Piazza, S Toth, J Pelliciari, R Fittipaldi, and A Vecchione. "Spin-orbitinduced orbital excitations in $\mathrm{Sr}_{2} \mathrm{RuO}_{4}$ and $\mathrm{Ca}_{2} \mathrm{RuO}_{4}$ : A resonant inelastic x-ray scattering study." In: Phys. Rev. B 91.15 (2015), p. 155104.

[511] Akira Chikamatsu, Yuji Kurauchi, Keisuke Kawahara, Tomoya Onozuka, Makoto Minohara, Hiroshi Kumigashira, Eiji Ikenaga, and Tetsuya Hasegawa. "Spectroscopic and theoretical investigation of the electronic states of layered perovskite oxyfluoride $\mathrm{Sr}_{2} \mathrm{RuO}_{3} \mathrm{~F}_{2}$ thin films." In: Phys. Rev. B 97.23 (2018), p. 235101.

[512] K Sun, Jingyue Liu, N Nag, and ND Browning. "Studying the metalsupport interaction in $\mathrm{Pd} / \gamma-\mathrm{Al}_{2} \mathrm{O}_{3}$ catalysts by atomic-resolution electron energy-loss spectroscopy." In: Catal. Lett. 84.3-4 (2002), pp. 193199 . 
[513] Masato Mogi, Yasuhide Inoue, Tomoyuki Yamamoto, Isao Tanaka, Ponnusamy Nachimuthu, and Rupert CC Perera. "Near-edge X-ray absorption fine structure of $\mathrm{PdO}$ at O K-edge." In: Jpn. J. Appl. Phys. 44.6R (2005), p. 4057.

[514] Valerii I Bukhtiyarov, Michael Hävecker, Vassily V Kaichev, Axel Knop-Gericke, Ralf W Mayer, and Robert Schlögl. "X-ray absorption and photoemission studies of the active oxygen for ethylene epoxidation over silver." In: Catal. Lett. 74.3-4 (2001), pp. 121-125.

[515] V V Kaichev, V I Bukhtiyarov, M Hävecker, A Knop-Gercke, R W Mayer, and R Schlögl. "The nature of electrophilic and nucleophilic oxygen adsorbed on silver." en. In: Kinet. Catal. $44 \cdot 3$ (2003), p. 9.

[516] Sergei Ph Ruzankin, Igor Zilberberg, and Georgii M Zhidomirov. "Closed and open-shell atomic oxygen on silver: two distinct patterns of the $\mathrm{O}$ is binding energy and X-ray absorption $\mathrm{O}$ K-edge spectra as revealed by density functional theory." In: Research on chemical intermediates 30.1 (2004), pp. 75-85.

[517] S Stemmer, ZQ Chen, WJ Zhu, and TP Ma. "Electron energy-loss spectroscopy study of thin film hafnium aluminates for novel gate dielectrics." In: J. Microsc. 210.1 (2003), pp. 74-79.

[518] Satoshi Toyoda, Jun Okabayashi, Hiroshi Kumigashira, Masaharu Oshima, Koichi Yamashita, Masaaki Niwa, Koji Usuda, and Guo-Lin Liu. "Crystallization in $\mathrm{HfO}_{2}$ gate insulators with in situ annealing studied by valence-band photoemission and x-ray absorption spectroscopy." In: J. Appl. Phys. 97.10 (2005), p. 104507.

[519] H Takahashi, J Okabayashi, S Toyoda, H Kumigashira, M Oshima, K Ikeda, GL Liu, Z Liu, and K Usuda. "Annealing-time dependence in interfacial reaction between poly-Si electrode and $\mathrm{HfO}_{2} / \mathrm{Si}$ gate stack studied by synchrotron radiation photoemission and $\mathrm{x}$-ray absorption spectroscopy." In: Appl. Phys. Lett. 89.1 (2006), p. 012102.

[520] Deok-Yong Cho, Jae-Min Lee, S-J Oh, Hoyoung Jang, J-Y Kim, J-H Park, and A Tanaka. "Influence of oxygen vacancies on the electronic structure of $\mathrm{HfO}_{2}$ films." In: Phys. Rev. B 76.16 (2007), p. 165411.

[521] DH Hill, Robert A Bartynski, Nhan V Nguyen, Albert C Davydov, Deane Chandler-Horowitz, and Martin M Frank. "The relationship between local order, long range order, and sub-band-gap defects in hafnium oxide and hafnium silicate films." In: J. Appl. Phys. 103.9 (2008), p. 093712. 
[522] M Oshima, H Takahashi, J Okabayashi, S Toyoda, H Kumigashira, M Inoue, M Mizutani, and J Yugami. "Band offsets and chemical bonding states in N-plasma-treated HfSiON gate stacks studied by photoelectron spectroscopy and x-ray absorption spectroscopy." In: J. Appl. Phys. 100.3 (2006), p. 033709.

[523] Suhas Kumar, Catherine E Graves, John Paul Strachan, AL David Kilcoyne, Tolek Tyliszczak, Yoshio Nishi, and R Stanley Williams. "Inoperando synchronous time-multiplexed O K-edge x-ray absorption spectromicroscopy of functioning tantalum oxide memristors." In: J. Appl. Phys. 118.3 (2015), p. 034502.

[524] JC Jan, PD Babu, HM Tsai, CW Pao, JW Chiou, SC Ray, KP Krishna Kumar, WF Pong, M-H Tsai, and CA Jong. "Bonding properties and their relation to residual stress and refractive index of amorphous $\mathrm{Ta}$ $(\mathrm{N}, \mathrm{O})$ films investigated by $\mathrm{x}$-ray absorption spectroscopy." In: Appl. phys. Lett. 86.16 (2005), p. 161910.

[525] J Purans, A Kuzmin, Ph Parent, and C Laffon. "Study of the electronic structure of rhenium and tungsten oxides on the O K-edge." In: Phys. B 259 (1999), pp. 1157-1158.

[526] Tsung-Yeh Yang, Chung-Yi Wu, Meng-Hung Tsai, Hong-Ming Lin, Wen-Li Tsai, and Yeukuang Hwu. "Thermal effects on the structural properties of tungsten oxide nanoparticles." In: J. Nanopart. Res. 6.2 (2004), pp. 171-179.

[527] DZ Guo, K Yu-Zhang, A Gloter, GM Zhang, and ZQ Xue. "Synthesis and characterization of tungsten oxide nanorods." In: J. Mater. Res. 19.12 (2004), pp. 3665-3670.

[528] MR Field, DG McCulloch, SNH Lim, A Anders, VJ Keast, and RW Burgess. "The electronic structure of tungsten oxide thin films prepared by pulsed cathodic arc deposition and plasma-assisted pulsed magnetron sputtering." In: J. Phys.: Condens. Matter 20.17 (2008), p. 175216.

[529] B Chen, J Laverock, LFJ Piper, ARH Preston, SW Cho, A DeMasi, KE Smith, DO Scanlon, GW Watson, and RG Egdell. "The band structure of $\mathrm{WO}_{3}$ and non-rigid-band behaviour in $\mathrm{NaxWO}_{3}$ derived from soft x-ray spectroscopy and density functional theory." In: J. Phys.: Condens. Matter 25.16 (2013), p. 165501.

[530] MB Johansson, Paw T Kristiansen, Laurent Duda, GA Niklasson, and Lars Österlund. "Band gap states in nanocrystalline $\mathrm{WO}_{3}$ thin films studied by soft x-ray spectroscopy and optical spectrophotometry." In: J. Phys.: Condens. Matter 28.47 (2016), p. 475802. 
[531] Woo Jin Kim, So Yeun Kim, Choong H Kim, Chang Hee Sohn, OB Korneta, Seung Chul Chae, and Tae Won Noh. "Spin-orbit coupling induced band structure change and orbital character of epitaxial $\mathrm{IrO}_{2}$ films." In: Phys. Rev. B 93.4 (2016), p. 045104.

[532] Verena Pfeifer, Travis E Jones, Juan J Velasco Vélez, Rosa Arrigo, Simone Piccinin, Michael Hävecker, Axel Knop-Gericke, and Robert Schlögl. "In situ observation of reactive oxygen species forming on oxygen-evolving iridium surfaces." In: Chem. Sci. 8.3 (2017), pp. 21432149 .

[533] Viktoriia A Saveleva, Li Wang, Detre Teschner, Travis E Jones, Aldo S Gago, K Andreas Friedrich, Spyridon Zafeiratos, Robert Schloegl, and Elena R Savinova. "Operando evidence for a universal oxygen evolution mechanism on thermal and electrochemical iridium oxides." In: J. Phys. Chem. Lett. (2018).

[534] Xiaoran Liu, Yanwei Cao, B Pal, S Middey, M Kareev, Y Choi, P Shafer, D Haskel, E Arenholz, and J Chakhalian. "Synthesis and electronic properties of Ruddlesden-Popper strontium iridate epitaxial thin films stabilized by control of growth kinetics." In: Phys. Rev. Materials 1.7 (2017), p. 075004.

[535] Xingye Lu, Paul Olalde-Velasco, Yaobo Huang, Valentina Bisogni, Jonathan Pelliciari, Sara Fatale, Marcus Dantz, James G Vale, EC Hunter, and Johan Chang. "Dispersive magnetic and electronic excitations in iridate perovskites probed by oxygen K-edge resonant inelastic x-ray scattering." In: Phys. Rev. B 97.4 (2018), p. 041102.

[536] M J Guinel, N Brodusch, Y Verde Gomez, B Escobar-Morales, and R Gauvin. "Multi-walled carbon nanotubes decorated by platinum catalyst nanoparticles-Examination and microanalysis using scanning and transmission electron microscopies." In: J. Microsc. 252.1 (2013), pp. 49-57.

[537] Stefan G. Minasian et al. "Quantitative evidence for lanthanide-oxygen orbital mixing in $\mathrm{CeO}_{2}, \mathrm{PrO}_{2}$, and $\mathrm{TbO}_{2}$." In: J. Am. Chem. Soc. 139.49 (Dec. 2017), pp. 18052-18064. IssN: 0002-7863.

[538] Lionel Calmels, PE Coulon, and Sylvie Schamm-Chardon. "Calculated and experimental electron energy-loss spectra of $\mathrm{La}_{2} \mathrm{O}_{3}, \mathrm{La}(\mathrm{OH})_{3}$, and LaOF nanophases in high permittivity lanthanum-based oxide layers." In: Appl. phys. Lett. 98.24 (2011), p. 243116. 
[539] Jong Seok Jeong, Wangzhou Wu, Mehmet Topsakal, Guichuan Yu, Takao Sasagawa, Martin Greven, and K Andre Mkhoyan. “Decomposition of $\mathrm{La}_{2-\chi} \mathrm{Sr}_{x} \mathrm{CuO}_{4}$ into several $\mathrm{La}_{2} \mathrm{O}_{3}$ phases at elevated temperatures in ultrahigh vacuum inside a transmission electron microscope." In: Phys. Rev. Materials 2.5 (2018), p. 054801.

[540] Huifang $\mathrm{Xu}$ and Yifeng Wang. "Electron energy-loss spectroscopy (EELS) study of oxidation states of $\mathrm{Ce}$ and $\mathrm{U}$ in pyrochlore and uraninitenatural analogues for Pu-and U-bearing waste forms." In: J. Nucl. Mater. 265.1-2 (1999), pp. 117-123.

[541] LAJ Garvie and PR Buseck. "Determination of $\mathrm{Ce}^{4+} / \mathrm{Ce}^{3+}$ in electronbeam-damaged $\mathrm{CeO}_{2}$ by electron energy-loss spectroscopy." In: J. Phys. Chem. Solids 60.12 (1999), pp. 1943-1947.

[542] NA Braaten, A Borg, JK Grepstad, S Raaen, and MW Ruckman. “Oxygen K near-edge-structure for thin Ce oxide films." In: Solid State Commun. 77.9 (1991), pp. 731-734.

[543] F Jollet, T Petit, S Gota, N Thromat, M Gautier-Soyer, and A Pasturel. "The electronic structure of uranium dioxide: an oxygen K-edge X-ray absorption study." In: J. Phys.: Condens. Matter 9.43 (1997), p. 9393.

[544] ZY Wu, F Jollet, S Gota, N Thromat, M Gautier-Soyer, and T Petit. "Xray absorption at the oxygen $\mathrm{K}$ edge in cubic f oxides examined using a full multiple-scattering approach." In: J. Phys.: Condens. Matter 11.37 (1999), p. 7185.

[545] Xianqin Wang, Jonathan C. Hanson, Gang Liu, Jose A. Rodriguez, Ana Iglesias-Juez, and Marcos Fernandez-Garcia. "The behavior of mixed-metal oxides: Physical and chemical properties of bulk and nanoparticles of (Ce,Tb)O $\mathrm{O}_{y}$." In: J. Chem. Phys. 121.11 (Sept. 2004), pp. 5434-5444. ISSN: 0021-9606.

[546] JA Rodriguez, X Wang, Gang Liu, JC Hanson, Jan Hrbek, Charles HF Peden, A Iglesias-Juez, and M Fernández-García. "Physical and chemical properties of $\mathrm{Ce}_{1-\chi} \mathrm{Zr}_{\chi} \mathrm{O}_{2}$ nanoparticles and $\mathrm{Ce}_{1-\chi} \mathrm{Zr}_{\chi} \mathrm{O}_{2}$ surfaces." In: J. Mol. Catal. A: Chem. 228.1-2 (2005), pp. 11-19.

[547] D.R Mullins, S.H Overbury, and D.R Huntley. "Electron spectroscopy of single crystal and polycrystalline cerium oxide surfaces." en. In: Surf. Sci. 409.2 (July 1998), pp. 307-319. ISSN: 00396028.

[548] A Modin, M-T Suzuki, J Vegelius, Y Yun, D K Shuh, L Werme, J Nordgren, P M Oppeneer, and S M Butorin. " 5 f-Shell correlation effects in dioxides of light actinides studied by $\mathrm{O}$ is $\mathrm{x}$-ray absorption 
and emission spectroscopies and first-principles calculations." en. In: J. Phys.: Condens. Matter 27.31 (Aug. 2015), p. 315503. IssN: 0953-8984, 1361-648X.

[549] A. Modin, Y. Yun, M.-T. Suzuki, J. Vegelius, L. Werme, J. Nordgren, P. M. Oppeneer, and S. M. Butorin. "Indication of single-crystal $\mathrm{PuO}_{2}$ oxidation from $\mathrm{O}$ is x-ray absorption spectra." In: Phys. Rev. B 83.7 (Feb. 2011), p. 075113 .

[550] Elodie Dalodiere et al. "Insights into the sonochemical synthesis and properties of salt-free intrinsic plutonium colloids." en. In: Sci. Rep. 7 (Mar. 2017), p. 43514. ISSN: 2045-2322.

[551] Philippe Martin, Michel Ripert, Gaëlle Carlot, Philippe Parent, and Carine Laffon. "A study of molybdenum behaviour in $\mathrm{UO}_{2}$ by X-ray absorption spectroscopy." en. In: J. Nucl. Mater. 326.2-3 (Mar. 2004), pp. 132-143. ISSN: 00223115. (Visited on 03/21/2018).

[552] R. G. Denning, J. C. Green, T. E. Hutchings, C. Dallera, A. Tagliaferri, K. Giarda, N. B. Brookes, and L. Braicovich. "Covalency in the uranyl ion: A polarized x-ray spectroscopic study." en. In: J. Chem. Phys. 117.17 (Nov. 2002), pp. 8008-8020. ISSN: 0021-9606, 1089-7690.

[553] Elisabetta Pizzi. "Influence of oxygen partial pressure on defect concentrations and on oxygen diffusion in $\mathrm{UO}_{2+x}$." English. PhD thesis. Ecole Centrale de Paris, 2013.

[554] Clara Fillaux, Christophe Den Auwer, Dominique Guillaumont, David K. Shuh, and Tolek Tyliszczak. "Investigation of actinide compounds by coupling X-ray absorption spectroscopy and quantum chemistry." In: J. Alloys Compd. Proceedings of the Plutonium Futures - The Science 2006 Conference 444-445 (Oct. 2007), pp. 443-446. ISSN: 09258388 .

[555] Clara Fillaux et al. "Combining theoretical chemistry and XANES multi-edge experiments to probe actinide valence states." In: Comptes Rendus Chimie 10.10 (Oct. 2007), pp. 859-871. IssN: 1631-0748. 